\title{
Synthesis and Biological Evaluation of New Bicyclic Fluorinated Uracils Through Ring-Closing Metathesis
}

Santos Fustero, ${ }^{*},+, \dot{\dagger}$ Silvia Catalán, ${ }^{\dagger}$ Julio Piera,$^{\dagger}$ Juan F. Sanz-Cervera, ${ }^{\dagger, \grave{\dagger}}$ Begoña Fernández ${ }^{\dagger}$ and José Luis Aceña

\footnotetext{
†Departamento de Química Orgánica, Universidad de Valencia, E-46100 Burjassot, Spain, and †aboratorio de Moléculas Orgánicas, Centro de Investigación Príncipe Felipe, E-46013 Valencia, Spain
} santos.fustero@uv.es

\section{SUPPORTING INFORMATION}

\section{Table of contents:}

General methods $\quad$ S4

General procedure for the preparation of compounds $8 \quad$ S4

Data of 8a $\quad$ S4

Data of $\mathbf{8 b} \quad$ S5

Data of 8c S5

General procedure for the preparation of compounds $9 \quad$ S5

Data of 9a $\quad$ S6

Data of 9b S6

$\begin{array}{ll}\text { Data of 9c } & \text { S7 }\end{array}$

Data of 9d $\quad$ S7

Data of 9e S8

Data of 9f S8

Data of 9g S9

$\begin{array}{ll}\text { Data of } 9 h & \text { S9 }\end{array}$

$\begin{array}{ll}\text { Data of 9i } & \text { S10 }\end{array}$ 
$\begin{array}{ll}\text { Data of 9j } & \text { S10 }\end{array}$

$\begin{array}{ll}\text { Data of 9k } & \text { S11 }\end{array}$

$\begin{array}{ll}\text { Data of 9l } & \text { S11 }\end{array}$

$\begin{array}{ll}\text { Data of } \mathbf{4 c} & \mathrm{S} 12\end{array}$

$\begin{array}{ll}\text { Data of 4d } & \mathrm{S} 12\end{array}$

Data of 4e $\quad$ S13

Data of $\mathbf{4 f} \quad \mathrm{S} 13$

$\begin{array}{ll}\text { Data of } \mathbf{4 g} & \mathrm{S} 14\end{array}$

$\begin{array}{ll}\text { Data of 4c' } & \text { S14 }\end{array}$

$\begin{array}{ll}\text { Data of } \mathbf{4} \mathbf{e}^{\prime} & \mathrm{S} 15\end{array}$

Data of 12b $\quad$ S15

$\begin{array}{ll}\text { Data of 12c } & \text { S16 }\end{array}$

$\begin{array}{ll}\text { Data of 12d } & \text { S16 }\end{array}$

$\begin{array}{ll}\text { Data of 12e } & \text { S17 }\end{array}$

$\begin{array}{ll}\text { Data of } 5 \mathbf{b} & \mathrm{S} 17\end{array}$

$\begin{array}{ll}\text { Data of } 5 \mathbf{c} & \mathrm{S} 18\end{array}$

$\begin{array}{ll}\text { Data of 5d } & \text { S18 }\end{array}$

$\begin{array}{ll}\text { Data of 5e } & \text { S19 }\end{array}$

$\begin{array}{ll}\text { NMR spectra of 8a } & \text { S20-S22 }\end{array}$

$\begin{array}{ll}\text { NMR spectra of } \mathbf{8 b} & \text { S23-S25 }\end{array}$

$\begin{array}{ll}\text { NMR spectra of 8c } & \text { S26-S28 }\end{array}$

$\begin{array}{ll}\text { NMR spectra of 9a } & \text { S29-S31 }\end{array}$

NMR spectra of 9b S32-S34

$\begin{array}{ll}\text { NMR spectra of 9c S35-S37 } & \text { Sc }\end{array}$

$\begin{array}{ll}\text { NMR spectra of 9d } & \text { S38-S40 }\end{array}$

$\begin{array}{ll}\text { NMR spectra of 9e } & \text { S41-S43 }\end{array}$

NMR spectra of $9 f \quad$ S44-S46

$\begin{array}{ll}\text { NMR spectra of } \mathbf{9 g} & \text { S47-S49 }\end{array}$

$\begin{array}{ll}\text { NMR spectra of } 9 \mathbf{h} & \text { S50-S52 }\end{array}$

$\begin{array}{ll}\text { NMR spectra of 9i } & \text { S53-S55 }\end{array}$

$\begin{array}{ll}\text { NMR spectra of } \mathbf{9 j} & \text { S56-S58 }\end{array}$

$\begin{array}{ll}\text { NMR spectra of 9k S59-S61 } & \text { Sk }\end{array}$

$\begin{array}{ll}\text { NMR spectra of } 91 & \text { S62-S64 }\end{array}$ 
NMR spectra of $\mathbf{4 a}$

NMR spectra of $\mathbf{4 b}$

S68-S70

NMR spectra of 4c

S71-S73

NMR spectra of $\mathbf{4 d}$

S74-S76

NMR spectra of $\mathbf{4 e}$

S77-S79

NMR spectra of $\mathbf{4 f}$

S80-S82

NMR spectra of $\mathbf{4 g}$

S83-S85

NMR spectra of $\mathbf{4} \mathbf{b}^{\prime}$

S86-S88

NMR spectra of $\mathbf{4} \mathbf{c}^{\prime}$

S89-S91

NMR spectra of $\mathbf{4} \mathbf{e}^{\prime}$

S92-S94

NMR spectra of 12a

S95-S97

NMR spectra of $\mathbf{1 2 b}$

S98-S100

NMR spectra of 12c

S101-S103

NMR spectra of 12d

S104-S106

NMR spectra of 12e

S107-S109

NMR spectra of $\mathbf{5 a}$

S110-S112

NMR spectra of $\mathbf{5 b}$

S113-S115

NMR spectra of 5c

S116-S118

NMR spectra of $\mathbf{5 d}$

S119-S121

NMR spectra of 5e

S122-S124

NMR spectra of 5a'

S125-S127 
General methods. All reactions were carried out under argon or nitrogen atmosphere. The solvents were purified prior to use: THF and toluene were distilled from sodium/benzophenone, $\mathrm{CH}_{2} \mathrm{Cl}_{2}$ was distilled from calcium hydride. All reagents were used as received. The reactions were monitored with the aid of thin-layer chromatography (TLC) using $0.25 \mathrm{~mm}$ precoated silica gel plates. Visualization was carried out with UV light and aqueous ceric ammonium molybdate solution or potassium permanganate stain. Flash column chromatography was performed with the indicated solvents on silica gel 60 (particle size 0.040-0.063 mm). Melting points are uncorrected. Chemical shifts of NMR spectra are given in ppm $(\delta)$, referenced to the residual proton resonances of the solvents or fluorotrichloromethane in ${ }^{19} \mathrm{~F}$ NMR experiments. Coupling constants $(J)$ are given in Hertz $(\mathrm{Hz})$. The letters $\mathrm{m}, \mathrm{s}, \mathrm{d}, \mathrm{t}$, and $\mathrm{q}$ stand for multiplet, singlet, doublet, triplet, and quartet, respectively. The letters br indicate that the signal is broad.

General procedure for the preparation of compounds 8. $n$-Butyllithium (10.5 mmol, $2.5 \mathrm{M}$ in hexane) was slowly added to a solution of diisopropylamine $(10.5 \mathrm{mmol})$ in THF $(15 \mathrm{~mL})$ at $-30{ }^{\circ} \mathrm{C}$. The mixture was stirred at that temperature for $30 \mathrm{~min}$, after which time the temperature was lowered to $-50{ }^{\circ} \mathrm{C}$. A solution of the corresponding ester $2(8.4 \mathrm{mmol})$ in THF $(10 \mathrm{~mL})$ was then added dropwise and the reaction mixture was stirred until it took on a yellow color, which indicated the complete formation of the enolate. The temperature was then lowered to $-78{ }^{\circ} \mathrm{C}$ and a solution of the nitrile $\mathbf{6}^{1}(7.0 \mathrm{mmol})$ in THF $(10 \mathrm{~mL})$ was slowly added. After $c a .1-1.5 \mathrm{~h}$ it was quenched with a sat. aq. $\mathrm{NH}_{4} \mathrm{Cl}$ solution $(15 \mathrm{~mL})$ and extracted with EtOAc $(3 \times 20 \mathrm{~mL})$. The organic layers were pooled together, washed with brine, dried over anhydrous $\mathrm{Na}_{2} \mathrm{SO}_{4}$, and concentrated to give the crude product 8, which was purified by flash column chromatography (n-hexane:EtOAc, 4:1) on deactivated silica gel $\left(2 \% \mathrm{Et}_{3} \mathrm{~N}\right.$ in hexane $)$.

\section{Ethyl 3-amino-4,4-difluorohepta-2,6-dienoate (8a).}

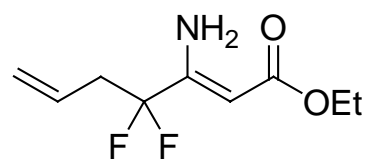

From ethyl acetate, enamino ester 8a was obtained as a yellow oil ( $95 \%$ yield). $R_{f}=0.48$ (n-hexane:EtOAc, 4:1). ${ }^{1} \mathrm{H}$ NMR $\left(300 \mathrm{MHz}, \mathrm{CDCl}_{3}\right) \delta 1.20(\mathrm{t}, J=7.0 \mathrm{~Hz}, 3 \mathrm{H}), 2.70$ (tdt, $J=16.0,7.2$, $1.5 \mathrm{~Hz}, 2 \mathrm{H}), 4.07$ (q, $J=7.2 \mathrm{~Hz}, 2 \mathrm{H}), 4.79$ (s, 1H), 5.17 (dd, $J=15.8,1.3 \mathrm{~Hz}, 1 \mathrm{H}), 5.18(\mathrm{dd}, J=10.3$, $0.6 \mathrm{~Hz}, 1 \mathrm{H}), 5.62-5.76(\mathrm{~m}, 1 \mathrm{H}) ;{ }^{13} \mathrm{C} \mathrm{NMR}\left(75.5 \mathrm{MHz}, \mathrm{CDCl}_{3}\right) \delta 13.4,40.6\left(\mathrm{t},{ }^{2} J_{\mathrm{CF}}=26.7 \mathrm{~Hz}\right), 58.4$, $83.3\left(\mathrm{t},{ }^{3} J_{\mathrm{CF}}=5.5 \mathrm{~Hz}\right), 117.8\left(\mathrm{t},{ }^{1} J_{\mathrm{CF}}=246.3 \mathrm{~Hz}\right), 120.4,126.6\left(\mathrm{t},{ }^{3} J_{\mathrm{CF}}=5.2 \mathrm{~Hz}\right), 153.9\left(\mathrm{t},{ }^{2} J_{\mathrm{CF}}=26.7\right.$ 
$\mathrm{Hz}), 168.9 ;{ }^{19} \mathrm{~F}$ NMR $\left(282.4 \mathrm{MHz}, \mathrm{CDCl}_{3}\right) \delta-104.9\left(\mathrm{t}, J_{\mathrm{HF}}=16.4 \mathrm{~Hz}, 2 \mathrm{~F}\right)$. HRMS (EI) calcd for $\mathrm{C}_{9} \mathrm{H}_{13} \mathrm{~F}_{2} \mathrm{NO}_{2}\left(\mathrm{M}^{+}\right)$: 205.0914, found: 205.0928 .

\section{Ethyl 3-amino-4,4-difluoro-2-vinylhepta-2,6-dienoate (8b).}<smiles>C=CCC(F)(F)/C(N)=C(\C=C)C(=O)OCC</smiles>

From ethyl but-3-enoate, enamino ester $8 \mathbf{b}$ was obtained as a yellow oil ( $30 \%$ yield). $R_{f}=0.49$ (n-hexane:EtOAc, 4:1). ${ }^{1} \mathrm{H}$ NMR $\left(300 \mathrm{MHz} \mathrm{CDCl}_{3}\right) \delta 1.25(\mathrm{t}, J=7.2 \mathrm{~Hz}, 3 \mathrm{H}), 2.85(\mathrm{td}, J=17.0,7.0$ $\mathrm{Hz}, 2 \mathrm{H}), 4.15(\mathrm{q}, J=7.2 \mathrm{~Hz}, 2 \mathrm{H}), 5.10(\mathrm{dd}, J=11.3,2.3 \mathrm{~Hz}, 1 \mathrm{H}), 5.16$ (d, $J=16.6 \mathrm{~Hz}, 1 \mathrm{H}), 5.17$ (d, $J=$ $9.8 \mathrm{~Hz}, 1 \mathrm{H}), 5.31(\mathrm{dt}, J=17.3,1.1 \mathrm{~Hz}, 1 \mathrm{H}), 5.61-5.74(\mathrm{~m}, 1 \mathrm{H}), 6.35$ (ddt, $J=17.5,11.3,1.5 \mathrm{~Hz}, 1 \mathrm{H})$, $6.92(\mathrm{br}, 2 \mathrm{H}) ;{ }^{13} \mathrm{C} \mathrm{NMR}\left(75.5 \mathrm{MHz}, \mathrm{CDCl}_{3}\right) \delta 14.2,41.2\left(\mathrm{t},{ }^{2} J_{\mathrm{CF}}=25.1 \mathrm{~Hz}\right), 60.0,96.0\left(\mathrm{t},{ }^{3} J_{\mathrm{CF}}=2.9\right.$ $\mathrm{Hz}), 116.7,119.7\left(\mathrm{t},{ }^{1} J_{\mathrm{CF}}=246.2 \mathrm{~Hz}\right), 121.3,127.5\left(\mathrm{t},{ }^{3} J_{\mathrm{CF}}=5.1 \mathrm{~Hz}\right), 129.0\left(\mathrm{t},{ }^{4} J_{\mathrm{CF}}=3.5 \mathrm{~Hz}\right), 152.2(\mathrm{t}$, $\left.{ }^{2} J_{\mathrm{CF}}=24.0 \mathrm{~Hz}\right), 170.2 ;{ }^{19} \mathrm{~F} \mathrm{NMR}\left(282.4 \mathrm{MHz}, \mathrm{CDCl}_{3}\right) \delta-98.7\left(\mathrm{t}, J_{\mathrm{HF}}=17.2 \mathrm{~Hz}, 2 \mathrm{~F}\right)$. HRMS (EI): calcd for $\mathrm{C}_{11} \mathrm{H}_{15} \mathrm{~F}_{2} \mathrm{NO}_{2}\left(\mathrm{M}^{+}\right)$: 231.1071, found: 231.1046 .

\section{Ethyl 2-allyl-3-amino-4,4-difluorohepta-2,6-dienoate (8c).}<smiles>C=CC/C(C(=O)OCC)=C(\N)C(F)(F)CC=C</smiles>

From ethyl pent-4-enoate, enamino ester 8c was obtained as a yellow oil (68\% yield). $R_{f}=0.47$ (n-hexane:EtOAc, 4:1). ${ }^{1} \mathrm{H}$ NMR $\left(300 \mathrm{MHz} \mathrm{CDCl}_{3}\right) \delta 1.17$ (t, J=7.1 Hz, 3H), 2.76 (tdt, J= 16.9, 7.1, $1.1 \mathrm{~Hz}, 2 \mathrm{H}), 2.94(\mathrm{dt}, J=5.6,1.5 \mathrm{~Hz}, 2 \mathrm{H}), 4.07$ (q, $J=7.1 \mathrm{~Hz}, 2 \mathrm{H}), 4.84-4.93(\mathrm{~m}, 2 \mathrm{H}), 5.14$ (dd, $J=$ 16.0, $1.5 \mathrm{~Hz}, 1 \mathrm{H}), 5.15(\mathrm{dd}, J=11.1,0.7 \mathrm{~Hz}, 1 \mathrm{H}), 5.62-5.81(\mathrm{~m}, 2 \mathrm{H}), 6.67(\mathrm{br}, 2 \mathrm{H}) ;{ }^{13} \mathrm{C}$ NMR $(75.5$ $\left.\mathrm{MHz}, \mathrm{CDCl}_{3}\right) \delta 14.7,30.7\left(\mathrm{t},{ }^{4} J_{\mathrm{CF}}=3.4 \mathrm{~Hz}\right), 42.2\left(\mathrm{t},{ }^{2} J_{\mathrm{CF}}=26.1 \mathrm{~Hz}\right), 60.0,93.9\left(\mathrm{t},{ }^{3} J_{\mathrm{CF}}=2.3 \mathrm{~Hz}\right)$, 114.5, $120.1\left(\mathrm{t},{ }^{1} J_{\mathrm{CF}}=247.4 \mathrm{~Hz}\right), 121.7,128.0\left(\mathrm{t},{ }^{3} J_{\mathrm{CF}}=4.9 \mathrm{~Hz}\right), 138.6,152.5\left(\mathrm{t},{ }^{2} J_{\mathrm{CF}}=24.1 \mathrm{~Hz}\right), 171.1$; ${ }^{19} \mathrm{~F} \mathrm{NMR}\left(282.4 \mathrm{MHz}, \mathrm{CDCl}_{3}\right) \delta-99.7\left(\mathrm{t}, J_{\mathrm{HF}}=17.0 \mathrm{~Hz}, 2 \mathrm{~F}\right)$. HRMS (EI) calcd for $\mathrm{C}_{12} \mathrm{H}_{17} \mathrm{~F}_{2} \mathrm{NO}_{2}\left(\mathrm{M}^{+}\right)$: 245.1227, found: 245.1204 .

General procedure for the preparation of compounds 9. $\mathrm{NaH}(3.6 \mathrm{mmol})$ was slowly added to a solution of $\beta$-enaminoester $8(2.0 \mathrm{mmol})$ in DMF $(3 \mathrm{ml})$ at $0{ }^{\circ} \mathrm{C}$. The mixture was stirred at that 
temperature for $30 \mathrm{~min}$, after which time phenyl isocyanate $(2.4 \mathrm{mmol})$ was added. The mixture was then stirred at room temperature for $c a .30 \mathrm{~min}$, then it was quenched with $\mathrm{H}_{2} \mathrm{O}$ and the solvent was removed under vacuum. The resulting crude mixture was taken up in $\mathrm{H}_{2} \mathrm{O}(10 \mathrm{ml})$ and washed with $\mathrm{CH}_{2} \mathrm{Cl}_{2}(3 \times 10 \mathrm{ml})$. The aqueous layer was concentrated to a volume of $2 \mathrm{ml}$ and then extracted with EtOAc $(3 \times 10 \mathrm{ml})$. The organic layers were pooled together, dried over anhydrous $\mathrm{Na}_{2} \mathrm{SO}_{4}$, and concentrated to give the crude product $\mathbf{9}$, which was purified in each case as described below.

\section{6-(1,1-Difluorobut-3-enyl)-3-phenylpyrimidine-2,4(1H,3H)-dione (9a).}<smiles>C=CCC(F)(F)c1cc(=O)n(-c2ccccc2)c(=O)[nH]1</smiles>

From enamino ester 8a, the crude product was washed with $n$-hexane- $\mathrm{CH}_{2} \mathrm{Cl}_{2}$ (5:1) to give pure $9 \mathbf{a}$ as a white solid (84\% yield). mp: 212-214 ${ }^{\circ} \mathrm{C} .{ }^{1} \mathrm{H}$ NMR $\left(300 \mathrm{MHz}, \mathrm{CD}_{3} \mathrm{OD}\right) \delta 2.84$ (td, J= 16.6, 7.0 Hz, 2H), $5.11(\mathrm{~d}, J=10.0 \mathrm{~Hz}, 1 \mathrm{H}), 5.15$ (d, $J=15.6 \mathrm{~Hz}, 1 \mathrm{H}), 5.64-5.72(\mathrm{~m}, 1 \mathrm{H}), 5.76(\mathrm{~s}, 1 \mathrm{H}), 7.07-7.10$ $(\mathrm{m}, 2 \mathrm{H}), 7.26-7.38(\mathrm{~m}, 3 \mathrm{H}) ;{ }^{13} \mathrm{C} \mathrm{NMR}\left(75.5 \mathrm{MHz}, \mathrm{CD}_{3} \mathrm{OD}\right) \delta 41.8\left(\mathrm{t},{ }^{2} J_{\mathrm{CF}}=25.6 \mathrm{~Hz}\right), 95.9\left(\mathrm{t},{ }^{3} J_{\mathrm{CF}}=\right.$ $5.7 \mathrm{~Hz}), 121.2,121.3\left(\mathrm{t},{ }^{1} J_{\mathrm{CF}}=243.4 \mathrm{~Hz}\right), 129.2,130.0,130.4,130.6\left(\mathrm{t},{ }^{3} J_{\mathrm{CF}}=5.2 \mathrm{~Hz}\right), 139.1,161.3$, $162.7\left(\mathrm{t},{ }^{2} J_{\mathrm{CF}}=27.9 \mathrm{~Hz}\right), 169.4 ;{ }^{19} \mathrm{~F}$ NMR $\left(282.4 \mathrm{MHz}, \mathrm{CD}_{3} \mathrm{OD}\right) \delta-104.9\left(\mathrm{t}, J_{\mathrm{HF}}=16.5 \mathrm{~Hz}, 2 \mathrm{~F}\right)$. IR (film): 3412, 1661, 1566, 1474, $1368 \mathrm{~cm}^{-1}$. HRMS (EI) calcd for $\mathrm{C}_{14} \mathrm{H}_{12} \mathrm{~F}_{2} \mathrm{~N}_{2} \mathrm{O}_{2}\left(\mathrm{M}^{+}\right): 278.0867$, found: 278.0837 .

\section{6-(1,1-Difluorobut-3-enyl)-3-phenyl-5-vinylpyrimidine-2,4(1H,3H)-dione (9b).}<smiles>C=CCC(F)(F)c1[nH]c(=O)n(-c2ccccc2)c(=O)c1C=C</smiles>

From enamino ester 8b, flash column chromatography (diisopropyl ether:EtOAc, 20:1) of the crude reaction mixture on silica gel gave $9 \mathbf{b}$ as a white solid (40\% yield). $R_{f}=0.23$ (diisopropyl ether:EtOAc, 20:1). mp: 108-110 ${ }^{\circ} \mathrm{C} .{ }^{1} \mathrm{H}$ NMR (300 MHz, $\left.\mathrm{CDCl}_{3}\right) \delta 2.95(\mathrm{td}, J=16.6,7.5 \mathrm{~Hz}, 2 \mathrm{H})$, 5.25 (dd, $J=15.8,2.6 \mathrm{~Hz}, 1 \mathrm{H}), 5.29$ (d, $J=9.0 \mathrm{~Hz}, 1 \mathrm{H}), 5.49$ (dd, $J=11.7,1.9 \mathrm{~Hz}, 1 \mathrm{H}), 5.61-5.75$ (m, 1H), 6.27 (dt, $J=15.8,1.1 \mathrm{~Hz}, 1 \mathrm{H}), 6.44(\mathrm{dd}, J=17.3,12.0 \mathrm{~Hz}, 1 \mathrm{H}), 7.31-7.42(\mathrm{~m}, 5 \mathrm{H}), 8.07$ (br, $1 \mathrm{H})$; ${ }^{13} \mathrm{C}$ NMR $\left(75.5 \mathrm{MHz}, \mathrm{CDCl}_{3}\right) \delta 40.3\left(\mathrm{t},{ }^{2} J_{\mathrm{CF}}=25.7 \mathrm{~Hz}\right), 108.7,117.7\left(\mathrm{t},{ }^{1} J_{\mathrm{CF}}=248.6 \mathrm{~Hz}\right), 121.9,122.2$, $123.3\left(\mathrm{t},{ }^{4} J_{\mathrm{CF}}=3.7 \mathrm{~Hz}\right), 125.0\left(\mathrm{t},{ }^{3} J_{\mathrm{CF}}=4.9 \mathrm{~Hz}\right), 127.1,128.3,133.1,139.7\left(\mathrm{t},{ }^{2} J_{\mathrm{CF}}=27.0 \mathrm{~Hz}\right), 148.2$, 
161.3; ${ }^{19} \mathrm{~F}$ NMR $\left(282.4 \mathrm{MHz}, \mathrm{CDCl}_{3}\right) \delta-98.3\left(\mathrm{t}, J_{\mathrm{HF}}=16.4 \mathrm{~Hz}, 2 \mathrm{~F}\right)$. IR (film): $3241,1709,1668$, 1493, $1419 \mathrm{~cm}^{-1}$. HRMS (EI): calcd for $\mathrm{C}_{16} \mathrm{H}_{14} \mathrm{~F}_{2} \mathrm{~N}_{2} \mathrm{O}_{2}\left(\mathrm{M}^{+}\right)$: 304.1023, found: 304.0979 .

5-Allyl-6-(1,1-difluorobut-3-enyl)-3-phenylpyrimidine-2,4(1H,3H)-dione (9c).<smiles>C=CCc1c(C(F)(F)CC=C)[nH]c(=O)n(-c2ccccc2)c1=O</smiles>

From enamino ester 8c, the crude product was washed with $n$-hexane- $\mathrm{CH}_{2} \mathrm{Cl}_{2}(5: 1)$ to give pure $9 \mathbf{c}$ as a white solid (62\% yield). mp: $183-185{ }^{\circ} \mathrm{C} .{ }^{1} \mathrm{H}$ NMR $\left(300 \mathrm{MHz}, \mathrm{CDCl}_{3}\right) \delta 2.84(\mathrm{td}, J=16.7,7.1 \mathrm{~Hz}$, 2H), 3.19 (dt, $J=6.4,1.3 \mathrm{~Hz}, 2 \mathrm{H}), 5.00(\mathrm{dd}, J=10.2,1.3 \mathrm{~Hz}, 1 \mathrm{H}), 5.05(\mathrm{dd}, J=18.3,1.3 \mathrm{~Hz}, 1 \mathrm{H}$ ), $5.18(\mathrm{~d}, J=17.1 \mathrm{~Hz}, 1 \mathrm{H}), 5.24(\mathrm{~d}, J=10.2 \mathrm{~Hz}, 1 \mathrm{H}), 5.58-5.69(\mathrm{~m}, 1 \mathrm{H}), 5.72-5.86(\mathrm{~m}, 1 \mathrm{H}), 7.14-7.17$ (m, 2H), 7.33-7.45 (m, 3H), $8.50(\mathrm{br}, 1 \mathrm{H}) ;{ }^{13} \mathrm{C} \mathrm{NMR}\left(75.5 \mathrm{MHz}, \mathrm{CDCl}_{3}\right) \delta 28.2\left(\mathrm{t},{ }^{4} \mathrm{~J}_{\mathrm{CF}}=2.9 \mathrm{~Hz}\right), 40.6$ $\left(\mathrm{t},{ }^{2} J_{\mathrm{CF}}=25.9 \mathrm{~Hz}\right), 110.3\left(\mathrm{t},{ }^{3} J_{\mathrm{CF}}=1.7 \mathrm{~Hz}\right), 115.7,117.6\left(\mathrm{t},{ }^{1} J_{\mathrm{CF}}=248.9 \mathrm{~Hz}\right), 122.0,125.1\left(\mathrm{t},{ }^{3} J_{\mathrm{CF}}=4.6\right.$ $\mathrm{Hz}), 127.0,127.9,128.3,133.1,133.4,140.1\left(\mathrm{t},{ }^{2} J_{\mathrm{CF}}=27.9 \mathrm{~Hz}\right), 149.2,162.3 ;{ }^{19} \mathrm{~F}$ NMR $(282.4 \mathrm{MHz}$, $\left.\mathrm{CDCl}_{3}\right) \delta-99.2\left(\mathrm{t}, J_{\mathrm{HF}}=17.0 \mathrm{~Hz}, 2 \mathrm{~F}\right.$ ). IR (film): 3082, 2982, 1721, 1649, 1497, $1427 \mathrm{~cm}^{-1}$. HRMS (EI): calcd for $\mathrm{C}_{17} \mathrm{H}_{16} \mathrm{~F}_{2} \mathrm{~N}_{2} \mathrm{O}_{2}\left(\mathrm{M}^{+}\right)$: 318.1180 , found: 318.1138 .

6-(1,1-Difluorobut-3-enyl)-3-(4-methoxyphenyl)pyrimidine-2,4(1H,3H)-dione (9d).<smiles>C=CCC(F)(F)c1cc(=O)n(-c2ccc(OC)cc2)c(=O)[nH]1</smiles>

The crude reaction product was washed with $n$-hexane- $\mathrm{CH}_{2} \mathrm{Cl}_{2}$ (5:1) to give pure $\mathbf{9 d}$ as a white solid (56\% yield). mp: $133-135^{\circ} \mathrm{C} .{ }^{1} \mathrm{H}$ NMR $\left(300 \mathrm{MHz}, \mathrm{CDCl}_{3}\right) \delta 2.79$ (td, J=15.0, 7.2 Hz, 2H), 3.77 (s, $3 \mathrm{H}), 5.16(\mathrm{dd}, J=17.0,5.1 \mathrm{~Hz}, 1 \mathrm{H}), 5.24(\mathrm{dd}, J=10.0,2.3 \mathrm{~Hz}, 1 \mathrm{H}), 5.57-5.72(\mathrm{~m}, 1 \mathrm{H}), 5.95(\mathrm{~s}, 1 \mathrm{H})$, $6.94(\mathrm{~d}, J=8.2 \mathrm{~Hz}, 2 \mathrm{H}), 7.07(\mathrm{~d}, J=8.4 \mathrm{~Hz}, 2 \mathrm{H}) ;{ }^{13} \mathrm{C} \mathrm{NMR}\left(75.5 \mathrm{MHz}, \mathrm{CDCl}_{3}\right) \delta 39.5\left(\mathrm{t},{ }^{2} J_{\mathrm{CF}}=25.9\right.$ $\mathrm{Hz}), 54.4,99.3\left(\mathrm{t},{ }^{3} J_{\mathrm{CF}}=4.6 \mathrm{~Hz}\right), 113.8,116.3\left(\mathrm{t},{ }^{1} J_{\mathrm{CF}}=246.6 \mathrm{~Hz}\right), 118.5,121.9,125.2\left(\mathrm{t},{ }^{3} J_{\mathrm{CF}}=5.8\right.$ $\mathrm{Hz}), 128.0,144.9\left(\mathrm{t},{ }^{2} J_{\mathrm{CF}}=30.5 \mathrm{~Hz}\right), 150.8,158.8,161.6 ;{ }^{19} \mathrm{~F} \mathrm{NMR}\left(282.4 \mathrm{MHz}, \mathrm{CDCl}_{3}\right) \delta-105.2(\mathrm{t}$, $J_{\mathrm{HF}}=19.6 \mathrm{~Hz}, 2 \mathrm{~F}$ ). IR (film): 3082, 2963, 2937, 2839, 1725, 1664, 1511, 1468, 1417, $1248 \mathrm{~cm}^{-1}$. HRMS (EI) calcd for $\mathrm{C}_{15} \mathrm{H}_{14} \mathrm{~F}_{2} \mathrm{~N}_{2} \mathrm{O}_{3}\left(\mathrm{M}^{+}\right)$: 308.0972 , found: 308.0967 . 
6-(1,1-Difluorobut-3-enyl)-3-(4-fluorophenyl)pyrimidine-2,4(1H,3H)-dione (9e).

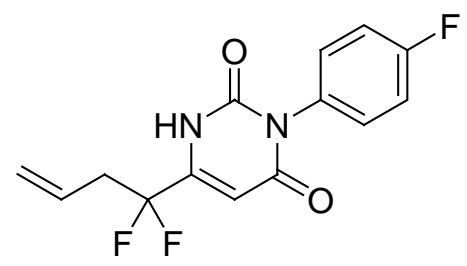

Flash chromatography ( $n$-hexane:EtOAc, 3:1) of the crude reaction mixture on silica gel gave 9e as a white solid (73\% yield). $R_{f}=0.28$ ( $n$-hexane:EtOAc, 3:1). mp: 187-189 ${ }^{\circ} \mathrm{C} .{ }^{1} \mathrm{H}$ NMR $(300 \mathrm{MHz}$, MeOD) $\delta 2.92(\mathrm{td}, J=16.6,7.2 \mathrm{~Hz}, 2 \mathrm{H}), 5.19(\mathrm{dd}, J=9.2,0.8 \mathrm{~Hz}, 1 \mathrm{H}), 5.23(\mathrm{dd}, J=14.3,1.3 \mathrm{~Hz}, 1 \mathrm{H})$ 5.69-5.81 (m, 1H), $5.84(\mathrm{~s}, 1 \mathrm{H}), 7.10-7.19(\mathrm{~m}, 4 \mathrm{H}), 7.92(\mathrm{br}, 1 \mathrm{H}) ;{ }^{13} \mathrm{C} \mathrm{NMR}(75.5 \mathrm{MHz}, \mathrm{MeOD}) \delta$ $41.7\left(\mathrm{t},{ }^{2} J_{\mathrm{CF}}=25.1 \mathrm{~Hz}\right), 100.4\left(\mathrm{t},{ }^{3} J_{\mathrm{CF}}=5.1 \mathrm{~Hz}\right), 117.5\left(\mathrm{~d},{ }^{2} J_{\mathrm{CF}}=22.8 \mathrm{~Hz}\right), 119.5\left(\mathrm{t},{ }^{1} J_{\mathrm{CF}}=243.8 \mathrm{~Hz}\right)$, $122.9,128.9\left(\mathrm{t},{ }^{3} J_{\mathrm{CF}}=5.2 \mathrm{~Hz}\right), 132.1\left(\mathrm{~d},{ }^{3} J_{\mathrm{CF}}=9.1 \mathrm{~Hz}\right), 132.4\left(\mathrm{~d},{ }^{4} J_{\mathrm{CF}}=3.1 \mathrm{~Hz}\right), 149.5\left(\mathrm{t},{ }^{2} J_{\mathrm{CF}}=30.3\right.$ $\mathrm{Hz}), 153.3,164.4\left(\mathrm{~d},{ }^{1} J_{\mathrm{CF}}=245.0 \mathrm{~Hz}\right), 165.4 ;{ }^{19} \mathrm{~F}$ NMR $(282.4 \mathrm{MHz}, \mathrm{MeOD}) \delta-126.9(\mathrm{~m}, 1 \mathrm{~F}),-114.8$ (t, $\left.J_{\mathrm{HF}}=16.3 \mathrm{~Hz}, 2 \mathrm{~F}\right)$. IR (film): 3107, 2978, 2857, 1738, 1650, 1514, $1428 \mathrm{~cm}^{-1}$. HRMS (EI) calcd for $\mathrm{C}_{14} \mathrm{H}_{11} \mathrm{~F}_{3} \mathrm{~N}_{2} \mathrm{O}_{2}\left(\mathrm{M}^{+}\right)$: 296.0773, found: 296.0763.

\section{(R)-6-(1,1-Difluorobut-3-enyl)-3-(1-phenylethyl)pyrimidine-2,4(1H,3H)-dione (9f).}<smiles>C=CCC(F)(F)c1cc(=O)n(C(C)c2ccccc2)c(=O)[nH]1</smiles>

Flash chromatography ( $n$-hexane:EtOAc, 2:1) of the crude reaction mixture on silica gel gave $\mathbf{9 f}$ as a white solid (67\% yield). $R_{f}=0.47$ ( $n$-hexane:EtOAc, $\left.2: 1\right)$. mp: $82-84{ }^{\circ} \mathrm{C}$. $[\alpha]^{25} \mathrm{D}+162.4$ (c 1.04, $\left.\mathrm{CHCl}_{3}\right) .{ }^{1} \mathrm{H} \mathrm{NMR}\left(300 \mathrm{MHz}, \mathrm{CDCl}_{3}\right) \delta 1.76(\mathrm{~d}, J=7.2 \mathrm{~Hz}, 3 \mathrm{H}), 2.59-2.86(\mathrm{~m}, 2 \mathrm{H}), 5.12(\mathrm{~d}, J=17.5$ $\mathrm{Hz}, 1 \mathrm{H}), 5.19$ (d, $J=10.2 \mathrm{~Hz}, 1 \mathrm{H}), 5.52-5.66(\mathrm{~m}, 1 \mathrm{H}), 5.84(\mathrm{~s}, 1 \mathrm{H}), 6.17$ (q, J= 7.2 Hz, 1H), 7.11-7.30 $(\mathrm{m}, 5 \mathrm{H}), 10.77(\mathrm{br}, 1 \mathrm{H}) ;{ }^{13} \mathrm{C} \mathrm{NMR}\left(75.5 \mathrm{MHz}, \mathrm{CDCl}_{3}\right) \delta 14.5,39.3\left(\mathrm{t},{ }^{2} J_{\mathrm{CF}}=25.8 \mathrm{~Hz}\right), 49.2,99.0(\mathrm{t}$, $\left.{ }^{3} J_{\mathrm{CF}}=5.2 \mathrm{~Hz}\right), 116.2\left(\mathrm{t},{ }^{1} J_{\mathrm{CF}}=246.6 \mathrm{~Hz}\right), 121.3,125.5\left(\mathrm{t},{ }^{3} J_{\mathrm{CF}}=5.7 \mathrm{~Hz}\right), 126.3,126.3,127.0,138.4$, $144.8\left(\mathrm{t},{ }^{2} J_{\mathrm{CF}}=31.0 \mathrm{~Hz}\right), 151.4,161.6 ;{ }^{19} \mathrm{~F} \mathrm{NMR}\left(282.4 \mathrm{MHz}, \mathrm{CDCl}_{3}\right) \delta-104.0\left(\mathrm{dt}, J_{\mathrm{FF}}=257.4 \mathrm{~Hz}\right.$, $\left.J_{\mathrm{HF}}=16.5 \mathrm{~Hz}, 1 \mathrm{~F}\right),-102.3\left(\mathrm{dt}, J_{\mathrm{FF}}=257.4 \mathrm{~Hz}, J_{\mathrm{HF}}=15.5 \mathrm{~Hz}, 1 \mathrm{~F}\right) . \mathrm{IR}$ (film): 3115, 2981, 1721, 1650, 1424, $1351 \mathrm{~cm}^{-1}$. HRMS (EI) calcd for $\mathrm{C}_{16} \mathrm{H}_{16} \mathrm{~F}_{2} \mathrm{~N}_{2} \mathrm{O}_{2}\left(\mathrm{M}^{+}\right)$: 306.1180, found: 306.1166 . 
6-(1,1-Difluorobut-3-enyl)-3-ethylpyrimidine-2,4(1H,3H)-dione (9g).<smiles>C=CCC(F)(F)c1cc(=O)n(CC)c(=O)[nH]1</smiles>

Flash chromatography ( $n$-hexane:EtOAc, 2:1) of the crude reaction mixture on silica gel gave $\mathbf{9 g}$ as a white solid (57\% yield). $R_{f}=0.33$ (n-hexane:EtOAc, 2:1). mp: 112-114 ${ }^{\circ} \mathrm{C} .{ }^{1} \mathrm{H}$ NMR $(300 \mathrm{MHz}$, $\left.\mathrm{CDCl}_{3}\right) \delta 1.17(\mathrm{t}, J=7.1 \mathrm{~Hz}, 3 \mathrm{H}), 2.85(\mathrm{td}, J=16.2,6.9 \mathrm{~Hz}, 2 \mathrm{H}), 3.92(\mathrm{q}, J=7.2 \mathrm{~Hz}, 2 \mathrm{H}), 5.23(\mathrm{~d}, J=$ $17.7 \mathrm{~Hz}, 1 \mathrm{H}), 5.25$ (dd, $J=9.4,1.1 \mathrm{~Hz}, 1 \mathrm{H}), 5.60-5.74(\mathrm{~m}, 1 \mathrm{H}), 5.85(\mathrm{~s}, 1 \mathrm{H}), 9.68(\mathrm{br}, 1 \mathrm{H}) ;{ }^{13} \mathrm{C} \mathrm{NMR}$ $\left(75.5 \mathrm{MHz}, \mathrm{CDCl}_{3}\right) \delta 11.6,35.1,39.5\left(\mathrm{t},{ }^{2} J_{\mathrm{CF}}=25.8 \mathrm{~Hz}\right), 99.0\left(\mathrm{t},{ }^{3} J_{\mathrm{CF}}=5.2 \mathrm{~Hz}\right), 116.3\left(\mathrm{t},{ }^{1} J_{\mathrm{CF}}=246.6\right.$ $\mathrm{Hz}), 121.6,125.4\left(\mathrm{t},{ }^{3} J_{\mathrm{CF}}=5.2 \mathrm{~Hz}\right), 144.5\left(\mathrm{t},{ }^{2} J_{\mathrm{CF}}=31.0 \mathrm{~Hz}\right), 151.1,161.2 ;{ }^{19} \mathrm{~F} \mathrm{NMR}(282.4 \mathrm{MHz}$, $\left.\mathrm{CDCl}_{3}\right) \delta-103.1\left(\mathrm{t}, J_{\mathrm{HF}}=16.4 \mathrm{~Hz}, 2 \mathrm{~F}\right) . \mathrm{IR}$ (film): 3099, 2984, 2874, 1728, 1640, $1449 \mathrm{~cm}^{-1}$. HRMS (EI) calcd for $\mathrm{C}_{10} \mathrm{H}_{12} \mathrm{~F}_{2} \mathrm{~N}_{2} \mathrm{O}_{2}\left(\mathrm{M}^{+}\right)$: 230.0867, found: 230.0837 .

\section{5-Allyl-6-(1,1-difluorobut-3-enyl)-3-(4-methoxyphenyl)pyrimidine-2,4(1H,3H)-dione (9h).}<smiles>C=CCc1c(C(F)(F)CC=C)[nH]c(=O)n(-c2ccc(OC)cc2)c1=O</smiles>

Flash chromatography ( $n$-hexane:EtOAc, 3:1) of the crude reaction mixture on silica gel gave $\mathbf{9 h}$ as a white solid (34\% yield). $R_{f}=0.30$ ( $n$-hexane:EtOAc, 3:1). mp: $108-110{ }^{\circ} \mathrm{C} .{ }^{1} \mathrm{H}$ NMR $(300 \mathrm{MHz}$, $\left.\mathrm{CDCl}_{3}\right) \delta 2.78(\mathrm{td}, J=16.6,7.0 \mathrm{~Hz}, 2 \mathrm{H}), 3.19(\mathrm{dd}, J=5.0,1.5 \mathrm{~Hz}, 2 \mathrm{H}), 3.76(\mathrm{~s}, 3 \mathrm{H}), 5.00(\mathrm{dd}, J=10.2$, $1.5 \mathrm{~Hz}, 1 \mathrm{H}), 5.05$ (dd, $J=17.3,1.5 \mathrm{~Hz}, 1 \mathrm{H}), 5.19$ (d, $J=17.1 \mathrm{~Hz}, 1 \mathrm{H}), 5.26$ (d, $J=10.2 \mathrm{~Hz}, 1 \mathrm{H}), 5.59$ $5.70(\mathrm{~m}, 1 \mathrm{H}), 5.70-5.86(\mathrm{~m}, 1 \mathrm{H}), 6.92(\mathrm{~d}, J=10.0 \mathrm{~Hz}, 2 \mathrm{H}), 7.07$ (d, J=10.0 Hz, 2H), 8.34 (br, 1H); ${ }^{13} \mathrm{C}$ NMR $\left(75.5 \mathrm{MHz}, \mathrm{CDCl}_{3}\right) \delta 28.3\left(\mathrm{t},{ }^{4} J_{\mathrm{CF}}=2.9 \mathrm{~Hz}\right), 40.7\left(\mathrm{t},{ }^{2} J_{\mathrm{CF}}=25.7 \mathrm{~Hz}\right), 54.5,110.2\left(\mathrm{~d},{ }^{3} J_{\mathrm{CF}}=\right.$ $1.7 \mathrm{~Hz}), 113.7,115.7,117.6\left(\mathrm{t},{ }^{1} J_{\mathrm{CF}}=247.3 \mathrm{~Hz}\right), 122.1,125.1\left(\mathrm{t},{ }^{3} J_{\mathrm{CF}}=4.6 \mathrm{~Hz}\right), 125.6,128.0,133.5$, $139.9\left(\mathrm{t},{ }^{2} J_{\mathrm{CF}}=28.0 \mathrm{~Hz}\right), 149.4,158.7,162.6 ;{ }^{19} \mathrm{~F}$ NMR $\left(282.4 \mathrm{MHz}, \mathrm{CDCl}_{3}\right) \delta-99.3\left(\mathrm{t}, J_{\mathrm{HF}}=16.4 \mathrm{~Hz}\right.$, 2F). IR (film): 3127, 3083, 1721, 1651, 1512, 1429, $1250 \mathrm{~cm}^{-1}$. HRMS (EI) calcd for $\mathrm{C}_{18} \mathrm{H}_{18} \mathrm{~F}_{2} \mathrm{~N}_{2} \mathrm{O}_{3}$ $\left(\mathrm{M}^{+}\right)$: 348.1285, found: 348.1377. 
5-Allyl-6-(1,1-difluorobut-3-enyl)-3-(4-fluorophenyl)pyrimidine-2,4(1H,3H)-dione (9i).

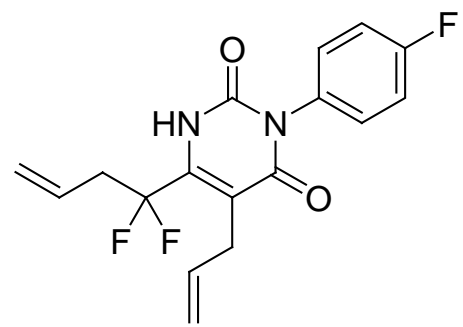

Flash chromatography ( $n$-hexane:EtOAc, 3:1) of the crude reaction mixture on silica gel gave 9i as a white solid (33\% yield). $R_{f}=0.34$ ( $n$-hexane:EtOAc, 3:1). mp: 110-112 ${ }^{\circ} \mathrm{C} .{ }^{1} \mathrm{H}$ NMR $(300 \mathrm{MHz}$, $\left.\mathrm{CDCl}_{3}\right) \delta 2.89(\mathrm{td}, J=16.8,7.2 \mathrm{~Hz}, 2 \mathrm{H}), 3.20(\mathrm{dt}, J=6.2,1.4 \mathrm{~Hz}, 2 \mathrm{H}), 5.02(\mathrm{dd}, J=10.0,1.5 \mathrm{~Hz}, 1 \mathrm{H})$, $5.06(\mathrm{dd}, J=16.8,1.4 \mathrm{~Hz}, 1 \mathrm{H}), 5.24$ (d, $J=18.0 \mathrm{~Hz}, 1 \mathrm{H}), 5.29$ (d, $J=10.7 \mathrm{~Hz}, 1 \mathrm{H}), 5.63-5.75$ (m, 1H), 5.75-5.84 (m, 1H), 7.11-7.15 (m, 4H), $8.15(\mathrm{br}, 1 \mathrm{H}) ;{ }^{13} \mathrm{C} \mathrm{NMR}\left(100 \mathrm{MHz}, \mathrm{CDCl}_{3}\right) \delta 28.3\left(\mathrm{t},{ }^{4} J_{\mathrm{CF}}=2.4\right.$ $\mathrm{Hz}), 40.8\left(\mathrm{t},{ }^{2} J_{\mathrm{CF}}=26.2 \mathrm{~Hz}\right), 110.3\left(\mathrm{~d},{ }^{3} J_{\mathrm{CF}}=1.8 \mathrm{~Hz}\right), 115.5\left(\mathrm{~d},{ }^{2} J_{\mathrm{CF}}=23.2 \mathrm{~Hz}\right), 115.9,117.6\left(\mathrm{t},{ }^{1} J_{\mathrm{CF}}=\right.$ $248.0 \mathrm{~Hz}), 122.3,125.0\left(\mathrm{t},{ }^{3} J_{\mathrm{CF}}=4.3 \mathrm{~Hz}\right), 128.8\left(\mathrm{~d},{ }^{4} J_{\mathrm{CF}}=3.7 \mathrm{~Hz}\right), 129.0\left(\mathrm{~d},{ }^{3} J_{\mathrm{CF}}=9.1 \mathrm{~Hz}\right), 133.3$, $140.0\left(\mathrm{t},{ }^{2} J_{\mathrm{CF}}=28.0 \mathrm{~Hz}\right), 148.8,161.6\left(\mathrm{~d},{ }^{1} J_{\mathrm{CF}}=247.4 \mathrm{~Hz}\right), 162.3 ;{ }^{19} \mathrm{~F}$ NMR $\left(282.4 \mathrm{MHz}, \mathrm{CDCl}_{3}\right) \delta-$ 112.6 (m, 1F), -99.3 (t, $\left.J_{\mathrm{HF}}=16.5 \mathrm{~Hz}, 2 \mathrm{~F}\right)$. IR (film): 3128, 3088, 1723, 1660, 1509, $1429 \mathrm{~cm}^{-1}$. HRMS (EI) calcd for $\mathrm{C}_{17} \mathrm{H}_{15} \mathrm{~F}_{3} \mathrm{~N}_{2} \mathrm{O}_{2}\left(\mathrm{M}^{+}\right)$: 336.1086, found: 336.1064 .

(R)-5-Allyl-6-(1,1-difluorobut-3-enyl)-3-(1-phenylethyl)pyrimidine-2,4(1H,3H)-dione (9j).<smiles>C=CCc1c(C(F)(F)CC=C)[nH]c(=O)n(C(C)c2ccccc2)c1=O</smiles>

Flash chromatography ( $n$-hexane:EtOAc, 5:1) of the crude reaction mixture on silica gel gave $\mathbf{9 j}$ as a yellow oil (72\% yield). $R_{f}=0.50$ ( $n$-hexane:EtOAc, $\left.5: 1\right)$. $[\alpha]^{25}{ }_{\mathrm{D}}+171.2\left(c 0.78, \mathrm{CHCl}_{3}\right) .{ }^{1} \mathrm{H}$ NMR $(300$ MHz, MeOD) $\delta 1.73(\mathrm{~d}, J=7.2 \mathrm{~Hz}, 3 \mathrm{H}), 2.85(\mathrm{td}, J=16.7,7.2 \mathrm{~Hz}, 2 \mathrm{H}), 3.10(\mathrm{dd}, J=5.8,1.6 \mathrm{~Hz}, 2 \mathrm{H})$, $4.86(\mathrm{dd}, J=9.1,1.5 \mathrm{~Hz}, 1 \mathrm{H}), 4.87$ (dd, $J=17.0,1.5 \mathrm{~Hz}, 1 \mathrm{H}), 5.14$ (dd, $J=17.3,1.1 \mathrm{~Hz}, 1 \mathrm{H}), 5.15$ (d, $J=10.9 \mathrm{~Hz}, 1 \mathrm{H}), 5.63-5.78(\mathrm{~m}, 2 \mathrm{H}), 6.10$ (q, $J=7.0 \mathrm{~Hz}, 1 \mathrm{H}), 7.07-7.16(\mathrm{~m}, 5 \mathrm{H}) ;{ }^{13} \mathrm{C} \mathrm{NMR}(100 \mathrm{MHz}$, $\left.\mathrm{CDCl}_{3}\right) \delta 14.7,28.1,40.7\left(\mathrm{t},{ }^{2} J_{\mathrm{CF}}=25.6 \mathrm{~Hz}\right), 49.9,109.9,115.1,117.6\left(\mathrm{t},{ }^{1} J_{\mathrm{CF}}=247.4 \mathrm{~Hz}\right), 121.6$, $125.4\left(\mathrm{t},{ }^{3} J_{\mathrm{CF}}=4.9 \mathrm{~Hz}\right), 126.3,126.4,127.1,133.8,138.6,140.1\left(\mathrm{t},{ }^{2} J_{\mathrm{CF}}=27.7 \mathrm{~Hz}\right), 149.7,162.4 ;{ }^{19} \mathrm{~F}$ NMR (282.4 MHz, $\left.\mathrm{CDCl}_{3}\right) \delta-99.7\left(\mathrm{t}, J_{\mathrm{HF}}=16.5 \mathrm{~Hz}, 2 \mathrm{~F}\right)$. IR (film): 3086, 2978, 1711, 1694, 1431, 1331, $1175 \mathrm{~cm}^{-1}$. HRMS (EI) calcd for $\mathrm{C}_{19} \mathrm{H}_{20} \mathrm{~F}_{2} \mathrm{~N}_{2} \mathrm{O}_{2}\left(\mathrm{M}^{+}\right)$: 346.1492, found: 346.1508 . 
5-Allyl-6-(1,1-difluorobut-3-enyl)-3-ethylpyrimidine-2,4(1H,3H)-dione (9k).<smiles>C=CCc1c(C(F)(F)CC=C)[nH]c(=O)n(CC)c1=O</smiles>

Flash chromatography ( $n$-hexane:EtOAc, 2.5:1) of the crude reaction mixture on silica gel gave 9k as a white solid (44\% yield). $R_{f}=0.34$ ( $n$-hexane:EtOAc, 2.5:1). mp: 67-69 ${ }^{\circ} \mathrm{C} .{ }^{1} \mathrm{H}$ NMR $(300 \mathrm{MHz}$, $\left.\mathrm{CDCl}_{3}\right) \delta 1.17(\mathrm{t}, J=7.2 \mathrm{~Hz}, 3 \mathrm{H}), 2.85(\mathrm{td}, J=16.6,7.2 \mathrm{~Hz}, 2 \mathrm{H}), 3.18(\mathrm{dt}, J=6.2,1.5 \mathrm{~Hz}, 2 \mathrm{H}), 3.93(\mathrm{q}$, $J=7.2 \mathrm{~Hz}, 2 \mathrm{H}), 5.00(\mathrm{dd}, J=10.0,1.5 \mathrm{~Hz}, 1 \mathrm{H}), 5.02$ (dd, $J=19.4,1.5 \mathrm{~Hz}, 1 \mathrm{H}), 5.21$ (dd, $J=15.6,0.7$ $\mathrm{Hz}, 1 \mathrm{H}), 5.25(\mathrm{~d}, J=8.5 \mathrm{~Hz}, 1 \mathrm{H}), 5.60-5.72(\mathrm{~m}, 1 \mathrm{H}), 5.72-5.86(\mathrm{~m}, 1 \mathrm{H}), 8.65(\mathrm{br}, 1 \mathrm{H}) ;{ }^{13} \mathrm{C}$ NMR $(75.5$

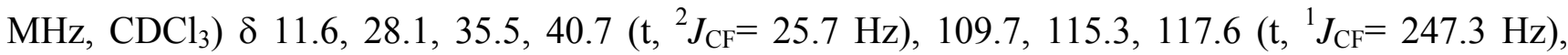
$121.8,125.3\left(\mathrm{t},{ }^{3} J_{\mathrm{CF}}=5.1 \mathrm{~Hz}\right), 133.8,139.7\left(\mathrm{t},{ }^{2} J_{\mathrm{CF}}=28.0 \mathrm{~Hz}\right), 149.5,162.0 ;{ }^{19} \mathrm{~F} \mathrm{NMR}(282.4 \mathrm{MHz}$, $\left.\mathrm{CDCl}_{3}\right) \delta-109.2\left(\mathrm{t}, J_{\mathrm{HF}}=17.3 \mathrm{~Hz}, 2 \mathrm{~F}\right) . \mathrm{IR}\left(\right.$ film): 3119, 3086, 2982, 1713, 1647, $1450 \mathrm{~cm}^{-1}$. HRMS (EI) calcd for $\mathrm{C}_{13} \mathrm{H}_{16} \mathrm{~F}_{2} \mathrm{~N}_{2} \mathrm{O}_{2}\left(\mathrm{M}^{+}\right): 270.1180$, found: 270.1178 .

5-Allyl-6-(1,1-difluorobut-3-enyl)-3-((ethoxycarbonyl)methyl)-pyrimidine-2,4(1H,3H)-dione (9l).<smiles>C=CCc1c(C(F)(F)CC=C)[nH]c(=O)n(CC(=O)OCC)c1=O</smiles>

Flash chromatography (n-hexane:EtOAc, 2:1) of the crude reaction mixture on silica gel gave 91 as a white solid (41\% yield). $R_{f}=0.29$ (n-hexane:EtOAc, $2: 1$ ). mp: $72-74{ }^{\circ} \mathrm{C} .{ }^{1} \mathrm{H}$ NMR $\left(300 \mathrm{MHz}, \mathrm{CDCl}_{3}\right)$ $\delta 1.21(\mathrm{t}, J=7.2 \mathrm{~Hz}, 3 \mathrm{H}), 2.85(\mathrm{td}, J=16.6,7.1 \mathrm{~Hz}, 2 \mathrm{H}), 3.19(\mathrm{dt}, J=6.0,1.5 \mathrm{~Hz}, 2 \mathrm{H}), 4.15(\mathrm{q}, J=7.2$ $\mathrm{Hz}, 2 \mathrm{H}), 4.60$ (s, 2H), 5.00 (dt, $J=10.5,1.5 \mathrm{~Hz}, 1 \mathrm{H}), 5.01$ (dd, $J=16.2,1.5 \mathrm{~Hz}, 1 \mathrm{H}), 5.22$ (dd, $J=15.8$, $0.8 \mathrm{~Hz}, 1 \mathrm{H}), 5.26(\mathrm{~d}, J=9.0 \mathrm{~Hz}, 1 \mathrm{H}), 5.59-5.75(\mathrm{~m}, 1 \mathrm{H}), 5.75-5.87(\mathrm{~m}, 1 \mathrm{H}) ;{ }^{13} \mathrm{C} \mathrm{NMR}(75.5 \mathrm{MHz}$, $\left.\mathrm{CDCl}_{3}\right) \delta 13.1,28.0\left(\mathrm{t},{ }^{4} J_{\mathrm{CF}}=3.4 \mathrm{~Hz}\right), 40.6\left(\mathrm{t},{ }^{2} J_{\mathrm{CF}}=25.1 \mathrm{~Hz}\right), 40.9,60.7,109.7,115.4,117.6\left(\mathrm{t},{ }^{1} J_{\mathrm{CF}}=\right.$ $247.3 \mathrm{~Hz}), 121.8,125.2\left(\mathrm{t},{ }^{3} J_{\mathrm{CF}}=4.6 \mathrm{~Hz}\right), 133.5,140.4\left(\mathrm{t},{ }^{2} J_{\mathrm{CF}}=28.0 \mathrm{~Hz}\right), 149.6,161.7,166.3 ;{ }^{19} \mathrm{~F}$ NMR (282.4 MHz, $\left.\mathrm{CDCl}_{3}\right) \delta-99.4$ (s, 2F). IR (film): 3135, 2984, 1720, 1652, 1448, $1207 \mathrm{~cm}^{-1}$. HRMS (EI) calcd for $\mathrm{C}_{15} \mathrm{H}_{18} \mathrm{~F}_{2} \mathrm{~N}_{2} \mathrm{O}_{4}\left(\mathrm{M}^{+}\right)$: 328.1235, found: 328.1270 . 
9,9-Difluoro-8,9-dihydro-3-(4-methoxyphenyl)-1H-cyclohepta[d]pyrimidine-2,4(3H,5H)-dione (4c).

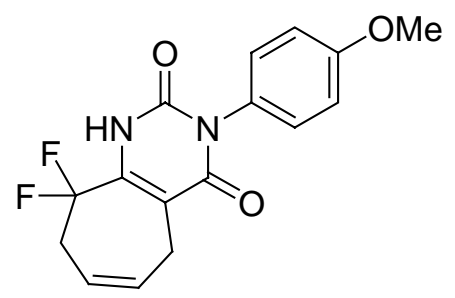

Flash chromatography ( $n$-hexane:EtOAc, 2:1) of the crude reaction mixture on silica gel gave $4 \mathbf{c}$ as a white solid (70\% yield). $R_{f}=0.31$ ( $n$-hexane:EtOAc, 2:1). mp: 134-136 ${ }^{\circ} \mathrm{C} .{ }^{1} \mathrm{H}$ NMR $(300 \mathrm{MHz}$, $\left.\mathrm{CDCl}_{3}\right) \delta 2.93(\mathrm{tdd}, J=15.1,5.8,1.3 \mathrm{~Hz}, 2 \mathrm{H}), 3.33-3-36(\mathrm{~m}, 2 \mathrm{H}), 3.76(\mathrm{~s}, 3 \mathrm{H}), 5.61-5-68(\mathrm{~m}, 1 \mathrm{H})$, 5.99-6.06 (m, 1H), $6.94(\mathrm{dd}, J=6.8,2.2 \mathrm{~Hz}, 2 \mathrm{H}), 7.07(\mathrm{dd}, J=6.8,2.2 \mathrm{~Hz}, 2 \mathrm{H}), 8.00(\mathrm{br}, 1 \mathrm{H}) ;{ }^{13} \mathrm{C}$ $\operatorname{NMR}\left(75.5 \mathrm{MHz}, \mathrm{CDCl}_{3}\right) \delta 21.9,33.8\left(\mathrm{t},{ }^{2} J_{\mathrm{CF}}=25.7 \mathrm{~Hz}\right), 54.5,111.1\left(\mathrm{t},{ }^{3} J_{\mathrm{CF}}=4.6 \mathrm{~Hz}\right), 113.8,115.1(\mathrm{t}$, $\left.{ }^{1} J_{\mathrm{CF}}=245.5 \mathrm{~Hz}\right), 120.5\left(\mathrm{t},{ }^{3} J_{\mathrm{CF}}=5.7 \mathrm{~Hz}\right), 125.9,128.0,130.3,139.8\left(\mathrm{t},{ }^{2} J_{\mathrm{CF}}=27.4 \mathrm{~Hz}\right), 149.1,158.8$, 162.4; ${ }^{19} \mathrm{~F}$ NMR $\left(282.4 \mathrm{MHz}, \mathrm{CDCl}_{3}\right) \delta-93.3\left(\mathrm{t}, J_{\mathrm{HF}}=14.6 \mathrm{~Hz}, 2 \mathrm{~F}\right) . \mathrm{IR}$ (film): 3128, 2963, 1721, 1650, 1510, 1419, $1257 \mathrm{~cm}^{-1}$. HRMS (EI) calcd for $\mathrm{C}_{16} \mathrm{H}_{14} \mathrm{~F}_{2} \mathrm{~N}_{2} \mathrm{O}_{3}\left(\mathrm{M}^{+}\right)$: 320.0972, found: 320.0890 .

9,9-Difluoro-8,9-dihydro-3-(4-fluorophenyl)-1H-cyclohepta[d]pyrimidine-2,4(3H,5H)-dione (4d).<smiles>O=c1[nH]c2c(c(=O)n1-c1ccc(F)cc1)CC=CCC2(F)F</smiles>

Flash chromatography ( $n$-hexane:EtOAc, 2:1) of the crude reaction mixture on silica gel gave $\mathbf{4 d}$ as a white solid (56\% yield). $R_{f}=0.42$ ( $n$-hexane:EtOAc, 2:1). mp: 188-190 ${ }^{\circ} \mathrm{C} .{ }^{1} \mathrm{H}$ NMR $(300 \mathrm{MHz}$, $\left.\mathrm{CDCl}_{3}\right) \delta 2.93(\mathrm{tdd}, J=15.1,5.6,1.3 \mathrm{~Hz}, 2 \mathrm{H}), 3.33-3.35(\mathrm{~m}, 2 \mathrm{H}), 5.61-5.69(\mathrm{~m}, 1 \mathrm{H}), 5.99-6.06(\mathrm{~m}$, 1H), 7.11-7.19 (m, 4H), $8.30(\mathrm{br}, 1 \mathrm{H}) ;{ }^{13} \mathrm{C} \mathrm{NMR}\left(75.5 \mathrm{MHz}, \mathrm{CDCl}_{3}\right) \delta 21.8,33.7\left(\mathrm{t},{ }^{2} J_{\mathrm{CF}}=25.8 \mathrm{~Hz}\right)$, $111.2\left(\mathrm{t},{ }^{3} J_{\mathrm{CF}}=5.2 \mathrm{~Hz}\right), 115.1\left(\mathrm{t},{ }^{1} J_{\mathrm{CF}}=244.3 \mathrm{~Hz}\right), 115.5\left(\mathrm{t},{ }^{2} J_{\mathrm{CF}}=23.1 \mathrm{~Hz}\right), 120.6\left(\mathrm{t},{ }^{3} J_{\mathrm{CF}}=6.0 \mathrm{~Hz}\right)$, $129.0\left(\mathrm{~d},{ }^{3} J_{\mathrm{CF}}=7.5 \mathrm{~Hz}\right), 129.1,130.2,140.0\left(\mathrm{t},{ }^{2} J_{\mathrm{CF}}=27.6 \mathrm{~Hz}\right), 148.8,161.6\left(\mathrm{~d},{ }^{1} J_{\mathrm{CF}}=249.0 \mathrm{~Hz}\right)$, 162.2; ${ }^{19} \mathrm{~F}$ NMR (282.4 MHz, $\left.\mathrm{CDCl}_{3}\right) \delta-112.7$ (s, 1F), -93.3 (s, 2F). IR (film): 3242, 3196, 1725, 1651, 1509, $1425 \mathrm{~cm}^{-1}$. HRMS (EI) calcd for $\mathrm{C}_{15} \mathrm{H}_{11} \mathrm{~F}_{3} \mathrm{~N}_{2} \mathrm{O}_{2}\left(\mathrm{M}^{+}\right)$: 308.0773, found: 308.0835 . 
(R)-9,9-Difluoro-8,9-dihydro-3-(1-phenylethyl)-1H-cyclohepta[d]pyrimidine-2,4(3H,5H)-dione

(4e).

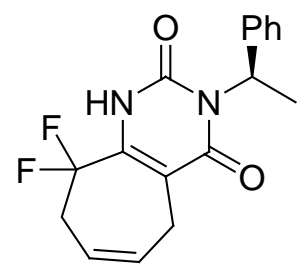

Flash chromatography ( $n$-hexane:diisopropyl ether, 1:2) of the crude reaction mixture on silica gel gave $4 \mathbf{e}$ as a colorless oil ( $82 \%$ yield). $R_{f}=0.25$ ( $n$-hexane:diisopropyl ether, $\left.1: 2\right)$. $[\alpha]_{\mathrm{D}}^{25}+93.2(c 1.3$, $\left.\mathrm{CHCl}_{3}\right) .{ }^{1} \mathrm{H} \mathrm{NMR}\left(300 \mathrm{MHz}, \mathrm{CDCl}_{3}\right) \delta 1.80(\mathrm{~d}, J=7.2 \mathrm{~Hz}, 3 \mathrm{H}), 2.87$ (tdd, $\left.J=14.3,6.0,1.5 \mathrm{~Hz}, 2 \mathrm{H}\right)$, 3.27-3.29 (m, 2H), 5.57-5.64 (m, 1H), 5.96-6.03 (m, 1H), 6.19 (q, J=7.2 Hz, 1H), 7.16-7.26 (m, 3H), 7.37-7.39 (d, 2H), $9.06(\mathrm{br}, 1 \mathrm{H}) ;{ }^{13} \mathrm{C} \mathrm{NMR}\left(100 \mathrm{MHz}, \mathrm{CDCl}_{3}\right) \delta 14.6,21.8,33.7\left(\mathrm{t},{ }^{2} J_{\mathrm{CF}}=25.6 \mathrm{~Hz}\right)$, $50.3,110.8\left(\mathrm{t},{ }^{3} J_{\mathrm{CF}}=4.9 \mathrm{~Hz}\right), 114.9\left(\mathrm{t},{ }^{1} J_{\mathrm{CF}}=242.5 \mathrm{~Hz}\right), 120.5\left(\mathrm{t},{ }^{3} J_{\mathrm{CF}}=5.8 \mathrm{~Hz}\right), 126.3,126.6,127.0$, 130.6, 138.6, $139.7\left(\mathrm{t},{ }^{2} J_{\mathrm{CF}}=27.1 \mathrm{~Hz}\right), 149.4,162.1 ;{ }^{19} \mathrm{~F} \mathrm{NMR}\left(282.4 \mathrm{MHz}, \mathrm{CDCl}_{3}\right) \delta-92.9\left(\mathrm{t}, J_{\mathrm{HF}}=\right.$ 14.6 Hz, 2F). HRMS (EI) calcd for $\mathrm{C}_{17} \mathrm{H}_{16} \mathrm{~F}_{2} \mathrm{~N}_{2} \mathrm{O}_{2}\left(\mathrm{M}^{+}\right)$: 318.1180 , found: 318.1166 .

\section{9,9-Difluoro-8,9-dihydro-3-ethyl-1H-cyclohepta[d]pyrimidine-2,4(3H,5H)-dione (4f).}

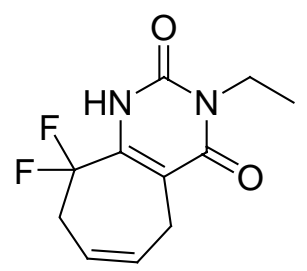

Flash chromatography ( $n$-hexane:EtOAc, 2:1) of the crude reaction mixture on silica gel gave $\mathbf{4 f}$ as a white solid, which was crystallized in $n$-hexane-ethanol (64\% yield). $R_{f}=0.37$ ( $n$-hexane:EtOAc, $2: 1$ ). mp: $156-158{ }^{\circ} \mathrm{C} .{ }^{1} \mathrm{H}$ NMR $\left(300 \mathrm{MHz}, \mathrm{CDCl}_{3}\right) \delta 1.17(\mathrm{t}, J=7.2 \mathrm{~Hz}, 3 \mathrm{H}), 2.89$ (tdd, $J=14.9,5.6,1.3$ $\mathrm{Hz}, 2 \mathrm{H}), 3.31-3.34(\mathrm{~m}, 2 \mathrm{H}), 3.94(\mathrm{q}, J=7.0 \mathrm{~Hz}, 2 \mathrm{H}), 5.58-5.65(\mathrm{~m}, 1 \mathrm{H}), 5.97-6.06(\mathrm{~m}, 1 \mathrm{H}), 8.80$ (br, $1 \mathrm{H}) ;{ }^{13} \mathrm{C}$ NMR $\left(75.5 \mathrm{MHz}, \mathrm{CDCl}_{3}\right) \delta 13.0,23.1,35.2\left(\mathrm{t},{ }^{2} J_{\mathrm{CF}}=25.6 \mathrm{~Hz}\right), 37.1,112.1\left(\mathrm{t},{ }^{3} J_{\mathrm{CF}}=5.1 \mathrm{~Hz}\right)$, $116.5\left(\mathrm{t},{ }^{1} J_{\mathrm{CF}}=242.2 \mathrm{~Hz}\right), 121.9\left(\mathrm{t},{ }^{3} J_{\mathrm{CF}}=5.7 \mathrm{~Hz}\right), 131.9,140.9\left(\mathrm{t},{ }^{2} J_{\mathrm{CF}}=26.8 \mathrm{~Hz}\right), 150.8,163.2 ;{ }^{19} \mathrm{~F}$ NMR (282.4 MHz, $\left.\mathrm{CDCl}_{3}\right) \delta-93.1\left(\mathrm{t}, J_{\mathrm{HF}}=14.6 \mathrm{~Hz}, 2 \mathrm{~F}\right) . \mathrm{IR}$ (film): 3116, 3057, 2983, 1702, 1635, 1452, $1333 \mathrm{~cm}^{-1}$. HRMS (EI) calcd for $\mathrm{C}_{11} \mathrm{H}_{12} \mathrm{~F}_{2} \mathrm{~N}_{2} \mathrm{O}_{2}\left(\mathrm{M}^{+}\right)$: 242.0867, found: 242.0872 . 
9,9-Difluoro-8,9-dihydro-3-((ethoxycarbonyl)methyl)-1H-cyclohepta[d]pyrimidine-2,4(1H,3H)dione (4g).<smiles>CCOC(=O)Cn1c(=O)[nH]c2c(c1=O)CC=CCC2(F)F</smiles>

Flash chromatography ( $n$-hexane:EtOAc, 2:1) of the crude reaction mixture on silica gel gave $\mathbf{4 g}$ as a colorless oil (80\% yield). $R_{f}=0.24$ ( $n$-hexane:EtOAc, $\left.2: 1\right)$. ${ }^{1} \mathrm{H}$ NMR $\left(300 \mathrm{MHz}, \mathrm{CDCl}_{3}\right) \delta 1.23(\mathrm{t}, J=$ $7.1 \mathrm{~Hz}, 3 \mathrm{H}), 2.90$ (tdd, $J=15.1,5.6,1.3 \mathrm{~Hz}, 2 \mathrm{H}), 3.31-3-33(\mathrm{~m}, 2 \mathrm{H}), 4.16$ (q, J= 7.5 Hz, 2H), 4.62 (s, 2H), 5.58-5.65 (m, 1H), 5.96-6.03 (m, 1H), 8.71 (br, 1H); $\left.{ }^{13} \mathrm{C} \mathrm{NMR} \mathrm{(75.5} \mathrm{MHz,} \mathrm{CDCl}_{3}\right) \delta 14.0,22.7$, $34.7\left(\mathrm{t},{ }^{2} J_{\mathrm{CF}}=25.7 \mathrm{~Hz}\right), 42.1,61.7,111.5\left(\mathrm{t},{ }^{3} J_{\mathrm{CF}}=5.1 \mathrm{~Hz}\right), 116.0\left(\mathrm{t},{ }^{1} J_{\mathrm{CF}}=242.0 \mathrm{~Hz}\right), 121.5\left(\mathrm{t},{ }^{3} J_{\mathrm{CF}}=\right.$ $6.3 \mathrm{~Hz}), 131.1,141.1\left(\mathrm{t},{ }^{2} J_{\mathrm{CF}}=27.4 \mathrm{~Hz}\right), 150.2,162.6,167.4 ;{ }^{19} \mathrm{~F}$ NMR $\left(282.4 \mathrm{MHz}, \mathrm{CDCl}_{3}\right) \delta-93.2$ $\left(\mathrm{t}, J_{\mathrm{HF}}=16.4 \mathrm{~Hz}, 2 \mathrm{~F}\right) . \mathrm{IR}$ (film): 3253, 2984, 1721, 1653, 1445, $1209 \mathrm{~cm}^{-1}$. HRMS (EI) calcd for $\mathrm{C}_{13} \mathrm{H}_{14} \mathrm{~F}_{2} \mathrm{~N}_{2} \mathrm{O}_{4}\left(\mathrm{M}^{+}\right)$: 300.0922, found: 300.0885 .

\section{9,9-Difluoro-8,9-dihydro-3-(4-methoxyphenyl)-1H-cyclohepta[d]pyrimidine-2,4(3H,7H)-dione} (4c').

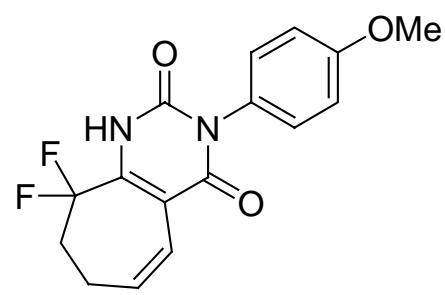

Flash chromatography ( $n$-hexane:EtOAc, $2: 1$ ) of the crude reaction mixture on silica gel gave 4c' as a white solid (88\% yield). $R_{f}=0.30$ ( $n$-hexane:EtOAc, 2:1). mp: 190-192 ${ }^{\circ} \mathrm{C} .{ }^{1} \mathrm{H}$ NMR $(300 \mathrm{MHz}$, $\left.\mathrm{CDCl}_{3}\right) \delta 2.40-2.59(\mathrm{~m}, 4 \mathrm{H}), 3.84(\mathrm{~s}, 3 \mathrm{H}), 6.02(\mathrm{dt}, J=12.8,4.5 \mathrm{~Hz}, 1 \mathrm{H}), 6.79(\mathrm{dt}, J=12.8,2.3 \mathrm{~Hz}$, $1 \mathrm{H}), 7.01(\mathrm{~d}, J=9.2 \mathrm{~Hz}, 2 \mathrm{H}), 7.15(\mathrm{~d}, J=9.0 \mathrm{~Hz}, 2 \mathrm{H}), 8.40(\mathrm{br}, 1 \mathrm{H}) ;{ }^{13} \mathrm{C} \mathrm{NMR}\left(75.5 \mathrm{MHz}, \mathrm{CDCl}_{3}\right) \delta$ $23.7\left(\mathrm{t},{ }^{3} J_{\mathrm{CF}}=6.9 \mathrm{~Hz}\right), 32.8\left(\mathrm{t},{ }^{2} J_{\mathrm{CF}}=24.1 \mathrm{~Hz}\right), 55.9,109.6\left(\mathrm{t},{ }^{3} J_{\mathrm{CF}}=5.7 \mathrm{~Hz}\right), 115.2,118.4\left(\mathrm{t},{ }^{1} J_{\mathrm{CF}}=\right.$ $243.7 \mathrm{~Hz}), 119.6,127.2,129.4,131.7,140.2\left(\mathrm{t},{ }^{2} J_{\mathrm{CF}}=26.4 \mathrm{~Hz}\right), 150.0,160.2,163.5 ;{ }^{19} \mathrm{~F} \mathrm{NMR}(282.4$ $\left.\mathrm{MHz}, \mathrm{CDCl}_{3}\right) \delta-93.5\left(\mathrm{t}, J_{\mathrm{FH}}=13.8 \mathrm{~Hz}, 2 \mathrm{~F}\right) . \mathrm{IR}$ (film): 3118, 1724, 1648, 1514, $1254 \mathrm{~cm}^{-1}$. HRMS (EI) calcd for $\mathrm{C}_{16} \mathrm{H}_{14} \mathrm{~F}_{2} \mathrm{~N}_{2} \mathrm{O}_{3}\left(\mathrm{M}^{+}\right)$: 320.0972, found: 320.0964 . 
(R)-9,9-Difluoro-8,9-dihydro-3-(1-phenylethyl)-1H-cyclohepta[d]pyrimidine-2,4(3H,7H)-dione (4e').

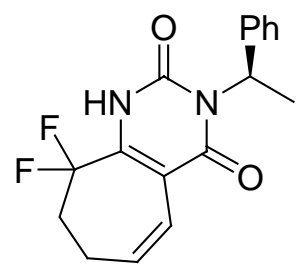

Flash chromatography (diisopropyl ether:EtOAc, 2:1) of the crude reaction mixture on silica gel gave 4e' as a white solid (74\% yield). $R_{f}=0.25$ (diisopropyl ether:EtOAc, $2: 1$ ). mp: $183-185^{\circ} \mathrm{C} .[\alpha]^{25}$

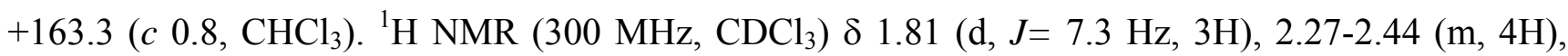
$5.90(\mathrm{dt}, J=12.4,4.9 \mathrm{~Hz}, 1 \mathrm{H}), 6.21$ (q, $J=7.2 \mathrm{~Hz}, 1 \mathrm{H}), 6.56$ (d, $J=12.4 \mathrm{~Hz}, 1 \mathrm{H}), 7.16-7.26(\mathrm{~m}, 3 \mathrm{H})$, $7.39(\mathrm{~d}, J=7.1 \mathrm{~Hz}, 2 \mathrm{H}), 9.01(\mathrm{br}, 1 \mathrm{H}) ;{ }^{13} \mathrm{C} \mathrm{NMR}\left(75.5 \mathrm{MHz}, \mathrm{CDCl}_{3}\right) \delta 14.6,22.2\left(\mathrm{t},{ }^{3} J_{\mathrm{CF}}=6.3 \mathrm{~Hz}\right)$, $31.5\left(\mathrm{t},{ }^{2} J_{\mathrm{CF}}=24.4 \mathrm{~Hz}\right), 50.3,107.9\left(\mathrm{t},{ }^{3} J_{\mathrm{CF}}=6.0 \mathrm{~Hz}\right), 116.9\left(\mathrm{t},{ }^{1} J_{\mathrm{CF}}=243.8 \mathrm{~Hz}\right), 117.9,126.3,126.5$, 127.0, 129.9, 138.5, $138.6\left(\mathrm{t},{ }^{2} J_{\mathrm{CF}}=27.0 \mathrm{~Hz}\right), 148.5,161.7 ;{ }^{19} \mathrm{~F} \mathrm{NMR}\left(282.4 \mathrm{MHz}, \mathrm{CDCl}_{3}\right) \delta-93.4(\mathrm{t}$, $\left.J_{\mathrm{HF}}=13.4 \mathrm{~Hz}, 2 \mathrm{~F}\right)$. HRMS (EI) calcd for $\mathrm{C}_{17} \mathrm{H}_{16} \mathrm{~F}_{2} \mathrm{~N}_{2} \mathrm{O}_{2}\left(\mathrm{M}^{+}\right)$: 318.1180 , found: 318.1180 .

\section{1-Allyl-6-(1,1-difluorobut-3-enyl)-3-(4-methoxyphenyl)pyrimidine-2,4(1H,3H)-dione (12b).}<smiles>C=CCn1c(C(F)(F)CC=C)cc(=O)n(-c2ccc(OC)cc2)c1=O</smiles>

Flash chromatography ( $n$-hexane:EtOAc, 4:1 to 2:1) of the crude reaction mixture on silica gel gave 12b as a white solid (55\% yield). $R_{f}=0.30$ ( $n$-hexane:EtOAc, $\left.2: 1\right)$. mp: $76-78{ }^{\circ} \mathrm{C} .{ }^{1} \mathrm{H}$ NMR $(300 \mathrm{MHz}$, $\left.\mathrm{CDCl}_{3}\right) \delta 2.94(\mathrm{td}, J=16.8,7.0 \mathrm{~Hz}, 2 \mathrm{H}), 3.75(\mathrm{~s}, 3 \mathrm{H}), 4.54(\mathrm{~d}, J=5.6 \mathrm{~Hz}, 2 \mathrm{H}), 5.15-5.30(\mathrm{~m}, 4 \mathrm{H})$, 5.66-5.90 (m, 2H), $6.07(\mathrm{~s}, 1 \mathrm{H}), 6.93(\mathrm{~d}, J=9.8 \mathrm{~Hz}, 2 \mathrm{H}), 7.05(\mathrm{~d}, J=9.8 \mathrm{~Hz}, 2 \mathrm{H}) ;{ }^{13} \mathrm{C} \mathrm{NMR}(75.5$

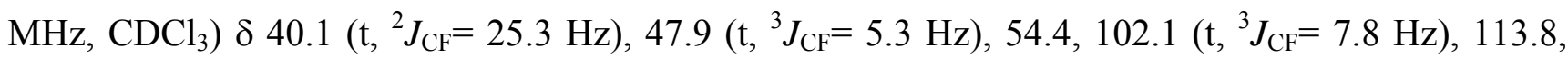
$117.3\left(\mathrm{t},{ }^{1} J_{\mathrm{CF}}=244.8 \mathrm{~Hz}\right), 117.7,121.4,125.7\left(\mathrm{t},{ }^{3} J_{\mathrm{CF}}=5.2 \mathrm{~Hz}\right), 126.1,127.9,130.8,145.7\left(\mathrm{t},{ }^{2} J_{\mathrm{CF}}=\right.$ $27.3 \mathrm{~Hz}$ ), 151.0, 158.7, 160.8; ${ }^{19} \mathrm{~F}$ NMR (282.4 MHz, $\left.\mathrm{CDCl}_{3}\right) \delta-97.6$ (s, 2F). IR (film): 1719, 1672 , 1511, 1451, 1382, $1249 \mathrm{~cm}^{-1}$. HRMS (EI) calcd for $\mathrm{C}_{18} \mathrm{H}_{18} \mathrm{~F}_{2} \mathrm{~N}_{2} \mathrm{O}_{3}\left(\mathrm{M}^{+}\right)$: 348.1285, found: 348.1350 . 
1-Allyl-6-(1,1-difluorobut-3-enyl)-3-(4-fluorophenyl)pyrimidine-2,4(1H,3H)-dione (12c).

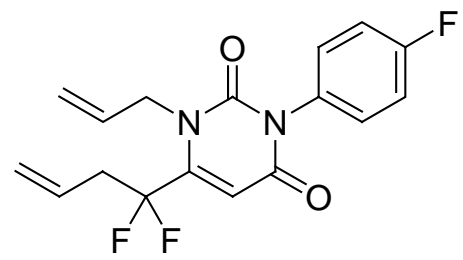

Flash chromatography ( $n$-hexane:EtOAc. $2: 1$ ) of the crude reaction mixture on silica gel gave 12c as a white solid (60\% yield). $R_{f}=0.35$ (n-hexane:EtOAc, $2: 1$ ). mp: $68-70{ }^{\circ} \mathrm{C} .{ }^{1} \mathrm{H}$ NMR $\left(300 \mathrm{MHz}, \mathrm{CDCl}_{3}\right)$ $\delta 2.95(\mathrm{td}, J=16.6,7.0 \mathrm{~Hz}, 2 \mathrm{H}), 4.54(\mathrm{~d}, J=5.5 \mathrm{~Hz}, 2 \mathrm{H}), 5.16-5.30$ (m, 4H), 5.66-5.90 (m, 2H), 6.08 (s, 1H), 7.10-7.15 (m, 4H); ${ }^{13} \mathrm{C}$ NMR $\left(75.5 \mathrm{MHz}, \mathrm{CDCl}_{3}\right) \delta 41.1\left(\mathrm{t},{ }^{2} J_{\mathrm{CF}}=25.1 \mathrm{~Hz}\right), 48.9\left(\mathrm{t},{ }^{3} J_{\mathrm{CF}}=4.9\right.$ $\mathrm{Hz}), 103.0\left(\mathrm{t},{ }^{3} J_{\mathrm{CF}}=8.0 \mathrm{~Hz}\right), 116.5\left(\mathrm{~d},{ }^{2} J_{\mathrm{CF}}=22.8 \mathrm{~Hz}\right), 118.2\left(\mathrm{t},{ }^{1} J_{\mathrm{CF}}=245.0 \mathrm{~Hz}\right), 118.8,122.5,126.6$ $\left(\mathrm{t},{ }^{3} J_{\mathrm{CF}}=5.1 \mathrm{~Hz}\right), 128.8\left(\mathrm{~d},{ }^{3} J_{\mathrm{CF}}=9.1 \mathrm{~Hz}\right), 129.3\left(\mathrm{~d},{ }^{4} J_{\mathrm{CF}}=3.4 \mathrm{~Hz}\right), 131.7,147.0\left(\mathrm{t},{ }^{2} J_{\mathrm{CF}}=27.4 \mathrm{~Hz}\right)$, 151.7, 161.5, $162.5\left(\mathrm{~d},{ }^{1} J_{\mathrm{CF}}=247.3 \mathrm{~Hz}\right) ;{ }^{19} \mathrm{~F} \mathrm{NMR}\left(282.4 \mathrm{MHz}, \mathrm{CDCl}_{3}\right) \delta-112.8(\mathrm{~m}, 1 \mathrm{~F}),-95.6(\mathrm{t}$, $J_{\mathrm{HF}}=17.2 \mathrm{~Hz}, 2 \mathrm{~F}$ ). IR (film): 1720, 1672, 1509, 1451, 1383, $1229 \mathrm{~cm}^{-1}$. HRMS (EI) calcd for $\mathrm{C}_{17} \mathrm{H}_{15} \mathrm{~F}_{3} \mathrm{~N}_{2} \mathrm{O}_{2}\left(\mathrm{M}^{+}\right)$: 336.1086, found: 336.1039 .

\section{(R)-1-Allyl-6-(1,1-difluorobut-3-enyl)-3-(1-phenylethyl)pyrimidine-2,4(1H,3H)-dione (12d).}

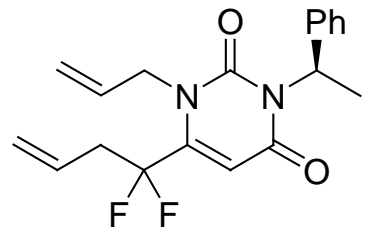

Flash chromatography ( $n$-hexane:EtOAc, $4: 1$ to $2: 1$ ) of the crude reaction mixture on silica gel gave 12d as a yellow oil (53\% yield). $R_{f}=0.40$ (n-hexane:EtOAc, $2: 1$ ). $[\alpha]^{25}{ }_{\mathrm{D}}+115.7$ (c 1.24, $\mathrm{CHCl}_{3}$ ). ${ }^{1} \mathrm{H}$ NMR (300 MHz, $\left.\mathrm{CDCl}_{3}\right) \delta 1.78(\mathrm{~d}, J=7.2 \mathrm{~Hz}, 3 \mathrm{H}), 2.85$ (td, $\left.J=16.8,7.0 \mathrm{~Hz}, 2 \mathrm{H}\right), 4.36-4.50$ (m, 2H), 5.00 (d, $J=17.3 \mathrm{~Hz}, 1 \mathrm{H}), 5.09$ (dd, $J=10.4,0.9 \mathrm{~Hz}, 1 \mathrm{H}), 5.19$ (d, $J=16.6 \mathrm{~Hz}, 1 \mathrm{H}), 5.23$ (d, $J=9.8 \mathrm{~Hz}$, $1 \mathrm{H}), 5.60-5.81(\mathrm{~m}, 2 \mathrm{H}), 5.92(\mathrm{~s}, 1 \mathrm{H}), 6.20(\mathrm{q}, J=7.1 \mathrm{~Hz}, 1 \mathrm{H}), 7.13-7.26(\mathrm{~m}, 3 \mathrm{H}), 7.31-7.33(\mathrm{~d}, 2 \mathrm{H})$; ${ }^{13} \mathrm{C}$ NMR $\left(75.5 \mathrm{MHz}, \mathrm{CDCl}_{3}\right) \delta 16.0,41.4\left(\mathrm{t},{ }^{2} J_{\mathrm{CF}}=25.1 \mathrm{~Hz}\right), 48.6\left(\mathrm{t},{ }^{4} J_{\mathrm{CF}}=5.1 \mathrm{~Hz}\right), 51.6,103.2(\mathrm{t}$, $\left.{ }^{3} J_{\mathrm{CF}}=8.0 \mathrm{~Hz}\right), 117.8,118.5\left(\mathrm{t},{ }^{1} J_{\mathrm{CF}}=244.5 \mathrm{~Hz}\right), 122.7,127.2\left(\mathrm{t},{ }^{3} J_{\mathrm{CF}}=5.1 \mathrm{~Hz}\right), 127.6,128.5,132.6$, $140.1,146.7\left(\mathrm{t},{ }^{2} J_{\mathrm{CF}}=27.4 \mathrm{~Hz}\right), 151.4,162.2 ;{ }^{19} \mathrm{~F} \mathrm{NMR}\left(282.4 \mathrm{MHz}, \mathrm{CDCl}_{3}\right) \delta-95.9\left(\mathrm{t}, J_{\mathrm{HF}}=16.4 \mathrm{~Hz}\right.$, 2F). IR (film): 1715, 1669, 1452, $1340 \mathrm{~cm}^{-1}$. HRMS (EI) calcd for $\mathrm{C}_{19} \mathrm{H}_{20} \mathrm{~F}_{2} \mathrm{~N}_{2} \mathrm{O}_{2}\left(\mathrm{M}^{+}\right): 346.1493$, found: 346.1454 . 


\section{1-Allyl-6-(1,1-difluorobut-3-enyl)-3-ethylpyrimidine-2,4(1H,3H)-dione (12e).}<smiles>C=CCn1c(C(F)(F)CC=C)cc(=O)n(CC)c1=O</smiles>

Flash chromatography ( $n$-hexane:EtOAc, 4:1) of the crude reaction mixture on silica gel gave 12e as a colorless oil (77\% yield). $R_{f}=0.30$ ( $n$-hexane:EtOAc, $\left.4: 1\right) .{ }^{1} \mathrm{H}$ NMR $\left(300 \mathrm{MHz}, \mathrm{CDCl}_{3}\right) \delta 1.16(\mathrm{t}, J=$ $7.2 \mathrm{~Hz}, 3 \mathrm{H}), 2.89$ (td, $J=16.6,6.8 \mathrm{~Hz}, 2 \mathrm{H}), 3.94$ (q, $J=7.2 \mathrm{~Hz}, 2 \mathrm{H}), 4.51$ (d, J=5.3 Hz, 2H), 5.08-5.26 $(\mathrm{m}, 4 \mathrm{H}), 5.61-5.63(\mathrm{~m}, 1 \mathrm{H}), 5.75-5.88(\mathrm{~m}, 1 \mathrm{H}), 5.94(\mathrm{~s}, 1 \mathrm{H}) ;{ }^{13} \mathrm{C} \mathrm{NMR}\left(75.5 \mathrm{MHz}, \mathrm{CDCl}_{3}\right) \delta 11.6$, $35.9,40.4\left(\mathrm{t},{ }^{2} J_{\mathrm{CF}}=25.2 \mathrm{~Hz}\right), 47.5\left(\mathrm{t},{ }^{4} J_{\mathrm{CF}}=5.1 \mathrm{~Hz}\right), 101.7\left(\mathrm{t},{ }^{3} J_{\mathrm{CF}}=7.4 \mathrm{~Hz}\right), 116.9,117.2\left(\mathrm{t},{ }^{1} J_{\mathrm{CF}}=\right.$ $244.5 \mathrm{~Hz}), 121.3,125.7\left(\mathrm{t},{ }^{3} J_{\mathrm{CF}}=5.1 \mathrm{~Hz}\right), 131.2,145.1\left(\mathrm{t},{ }^{2} J_{\mathrm{CF}}=26.8 \mathrm{~Hz}\right), 150.4,160.3 ;{ }^{19} \mathrm{~F} \mathrm{NMR}$ $\left(282.4 \mathrm{MHz}, \mathrm{CDCl}_{3}\right) \delta-105.7\left(\mathrm{t}, J_{\mathrm{HF}}=17.2 \mathrm{~Hz}, 2 \mathrm{~F}\right)$. IR (film): 1713, 1667, 1450, 1396, $1343 \mathrm{~cm}^{-1}$. HRMS (EI) calcd for $\mathrm{C}_{13} \mathrm{H}_{16} \mathrm{~F}_{2} \mathrm{~N}_{2} \mathrm{O}_{2}\left(\mathrm{M}^{+}\right): 270.1180$, found: 270.1186 .

\section{5,5-Difluoro-5,6-dihydro-2-(4-methoxyphenyl)pyrimido[1,6a]azepine-1,3(2H,9H)-dione (5b).}<smiles>COc1ccc(-n2c(=O)cc3n(c2=O)CC=CCC3(F)F)cc1</smiles>

Flash chromatography ( $n$-hexane:EtOAc, 2:1) of the crude reaction mixture on silica gel gave $5 \mathbf{b}$ as a white solid, which was crystallized in $n$-hexane-ethanol (93\% yield). $R_{f}=0.30$ ( $n$-hexane:EtOAc, $2: 1$ ). mp: 131-133 ${ }^{\circ} \mathrm{C} .{ }^{1} \mathrm{H}$ NMR (300 MHz, $\left.\mathrm{CDCl}_{3}\right) \delta 2.99$ (tq, J=15.8, $\left.2.1 \mathrm{~Hz}, 2 \mathrm{H}\right), 3.76$ (s, 3H), 4.72 (dd, $J=5.9,1.3 \mathrm{~Hz}, 2 \mathrm{H}), 5.62-5.66(\mathrm{~m}, 1 \mathrm{H}), 5.86-5.94(\mathrm{~m}, 1 \mathrm{H}), 6.18(\mathrm{~s}, 1 \mathrm{H}), 6.93$ (d, J=9.9 Hz, 2H), 7.06 $(\mathrm{d}, J=9.9 \mathrm{~Hz}, 2 \mathrm{H}) ;{ }^{13} \mathrm{C} \mathrm{NMR}\left(75.5 \mathrm{MHz}, \mathrm{CDCl}_{3}\right) \delta 37.6\left(\mathrm{t},{ }^{2} J_{\mathrm{CF}}=27.6 \mathrm{~Hz}\right), 39.7\left(\mathrm{t},{ }^{4} J_{\mathrm{CF}}=5.2 \mathrm{~Hz}\right)$, $54.4,99.5\left(\mathrm{t},{ }^{3} J_{\mathrm{CF}}=8.6 \mathrm{~Hz}\right), 113.8,116.1\left(\mathrm{t},{ }^{1} J_{\mathrm{CF}}=244.8 \mathrm{~Hz}\right), 123.7,123.8\left(\mathrm{t},{ }^{3} J_{\mathrm{CF}}=6.3 \mathrm{~Hz}\right), 126.4$, $127.9,147.1\left(\mathrm{t},{ }^{2} J_{\mathrm{CF}}=28.7 \mathrm{~Hz}\right), 150.7,158.7,161.0 ;{ }^{19} \mathrm{~F}$ NMR $\left(282.4 \mathrm{MHz}, \mathrm{CDCl}_{3}\right) \delta-97.2(\mathrm{t}, J=15.5$ $\mathrm{Hz}, 2 \mathrm{~F}$ ). IR (film): 1721, 1677, 1512, 1375, $1251 \mathrm{~cm}^{-1}$. HRMS (EI) calcd for $\mathrm{C}_{16} \mathrm{H}_{14} \mathrm{~F}_{2} \mathrm{~N}_{2} \mathrm{O}_{3}\left(\mathrm{M}^{+}\right)$: 320.0972, found: 320.1022 . 
5,5-Difluoro-5,6-dihydro-2-(4-fluorophenyl)pyrimido[1,6a]azepine-1,3(2H,9H)-dione (5c).<smiles>O=c1cc2n(c(=O)n1-c1ccc(F)cc1)CC=CCC2(F)F</smiles>

Flash chromatography ( $n$-hexane:EtOAc, 2:1) of the crude reaction mixture on silica gel gave 5c as a colorless oil (81\% yield). $R_{f}=0.32$ ( $n$-hexane:EtOAc, $\left.2: 1\right) .{ }^{1} \mathrm{H}$ NMR $\left(400 \mathrm{MHz}, \mathrm{CDCl}_{3}\right) \delta 3.00(\mathrm{td}, J=$ 16.0, $2.0 \mathrm{~Hz}, 2 \mathrm{H}), 4.71$ (d, J=5.2 Hz, 2H), 5.63-5.66 (m, 1H), 5.87-5.93 (m, 1H), $6.18(\mathrm{~s}, 1 \mathrm{H}), 7.10-$ $7.12(\mathrm{~m}, 4 \mathrm{H}) ;{ }^{13} \mathrm{C} \mathrm{NMR}\left(75.5 \mathrm{MHz}, \mathrm{CDCl}_{3}\right) \delta 38.6\left(\mathrm{t},{ }^{2} J_{\mathrm{CF}}=27.4 \mathrm{~Hz}\right), 40.7\left(\mathrm{t},{ }^{4} J_{\mathrm{CF}}=5.1 \mathrm{~Hz}\right), 100.4(\mathrm{t}$, $\left.{ }^{3} J_{\mathrm{CF}}=8.6 \mathrm{~Hz}\right), 116.6\left(\mathrm{~d},{ }^{2} J_{\mathrm{CF}}=22.8 \mathrm{~Hz}\right), 117.0\left(\mathrm{t},{ }^{1} J_{\mathrm{CF}}=243.9 \mathrm{~Hz}\right), 124.6,124.9\left(\mathrm{t},{ }^{3} J_{\mathrm{CF}}=6.3 \mathrm{~Hz}\right)$, $129.8\left(\mathrm{~d},{ }^{3} J_{\mathrm{CF}}=9.1 \mathrm{~Hz}\right), 130.7\left(\mathrm{~d},{ }^{4} J_{\mathrm{CF}}=3.4 \mathrm{~Hz}\right), 148.3\left(\mathrm{t},{ }^{2} J_{\mathrm{CF}}=28.0 \mathrm{~Hz}\right), 151.4,161.6,162.6\left(\mathrm{~d},{ }^{1} J_{\mathrm{CF}}=\right.$ $246.7 \mathrm{~Hz}) ;{ }^{19} \mathrm{~F}$ NMR $\left(282.4 \mathrm{MHz}, \mathrm{CDCl}_{3}\right) \delta-117.4(\mathrm{~m}, 1 \mathrm{~F}),-101.9$ (t, J=16.4 Hz, 2F). IR (film): 1719, 1672, 1509, 1468, 1386, 1350, $1247 \mathrm{~cm}^{-1}$. HRMS (EI) calcd for $\mathrm{C}_{15} \mathrm{H}_{11} \mathrm{~F}_{3} \mathrm{~N}_{2} \mathrm{O}_{2}\left(\mathrm{M}^{+}\right): 308.0773$, found: 308.0814 .

(R)-5,5-Difluoro-5,6-dihydro-2-(1-phenylethyl)pyrimido[1,6a]azepine-1,3(2H,9H)-dione (5d).<smiles>CC(c1ccccc1)n1c(=O)cc2n(c1=O)CC=CCC2(F)F</smiles>

Flash chromatography ( $n$-hexane:EtOAc, 2:1) of the crude reaction mixture on silica gel gave $\mathbf{5 d}$ as a colorless oil (90\% yield). $R_{f}=0.47$ ( $n$-hexane:EtOAc, $\left.2: 1\right)$. $[\alpha]^{25}+171.5\left(c 1, \mathrm{CHCl}_{3}\right) .{ }^{1} \mathrm{H}$ NMR $(300$ $\left.\mathrm{MHz}, \mathrm{CDCl}_{3}\right) \delta 1.78(\mathrm{~d}, J=7.2 \mathrm{~Hz}, 3 \mathrm{H}), 2.90(\mathrm{tq}, J=15.8,1.9 \mathrm{~Hz}, 2 \mathrm{H}), 4.51-4.67$ (m, 2H), 5.54-5.58 (m, 1H), 5.77-5.85 (m, 1H), $6.02(\mathrm{~s}, 1 \mathrm{H}), 6.23(\mathrm{q}, J=7.2 \mathrm{~Hz}, 1 \mathrm{H}), 7.17-7.27(\mathrm{~m}, 3 \mathrm{H}), 7.34-7.36(\mathrm{~d}$, $2 \mathrm{H}) ;{ }^{13} \mathrm{C} \mathrm{NMR}\left(75.5 \mathrm{MHz}, \mathrm{CDCl}_{3}\right) \delta 14.5,37.6\left(\mathrm{t},{ }^{2} J_{\mathrm{CF}}=27.9 \mathrm{~Hz}\right), 39.1\left(\mathrm{t},{ }^{4} J_{\mathrm{CF}}=4.6 \mathrm{~Hz}\right), 50.2,99.0(\mathrm{t}$, $\left.{ }^{3} J_{\mathrm{CF}}=8.6 \mathrm{~Hz}\right), 116.0\left(\mathrm{t},{ }^{1} J_{\mathrm{CF}}=243.3 \mathrm{~Hz}\right), 123.7\left(\mathrm{t},{ }^{3} J_{\mathrm{CF}}=6.3 \mathrm{~Hz}\right), 123.8,126.3,126.4,127.0,138.7$, $146.6\left(\mathrm{t},{ }^{2} J_{\mathrm{CF}}=28.6 \mathrm{~Hz}\right), 149.5,160.9 ;{ }^{19} \mathrm{~F} \mathrm{NMR}\left(282.4 \mathrm{MHz}, \mathrm{CDCl}_{3}\right) \delta-97.5(\mathrm{t}, J=15.5 \mathrm{~Hz}, 2 \mathrm{~F}) . \mathrm{IR}$ (film): 1711, 1664, 1470, 1343, $1288 \mathrm{~cm}^{-1}$. HRMS (EI) calcd for $\mathrm{C}_{17} \mathrm{H}_{16} \mathrm{~F}_{2} \mathrm{~N}_{2} \mathrm{O}_{2}\left(\mathrm{M}^{+}\right): 318.1180$, found: 318.1112 . 
5,5-Difluoro-5,6-dihydro-2-ethylpyrimido[1,6a]azepine-1,3(2H,9H)-dione (5e).<smiles>CCn1c(=O)cc2n(c1=O)CC=CCC2(F)F</smiles>

Flash chromatography ( $n$-hexane:EtOAc, 2:1) of the crude reaction mixture on silica gel gave 5e as a colorless oil (81\% yield). $R_{f}=0.45$ ( $n$-hexane:EtOAc, $\left.2: 1\right) .{ }^{1} \mathrm{H}$ NMR $\left(300 \mathrm{MHz}, \mathrm{CDCl}_{3}\right) \delta 1.16(\mathrm{t}, J=$ $7.0 \mathrm{~Hz}, 3 \mathrm{H}), 2.93$ (tq, $J=15.8,2.3 \mathrm{~Hz}, 2 \mathrm{H}), 3.94$ (q, $J=7.0 \mathrm{~Hz}, 2 \mathrm{H}), 4.69$ (dt, $J=4.4,1.5 \mathrm{~Hz}, 2 \mathrm{H})$, 5.58-5.63 (m, 1H), 5.84-5.93 (m, 1H), $6.03(\mathrm{~s}, 1 \mathrm{H}) ;{ }^{13} \mathrm{C} \mathrm{NMR}\left(75.5 \mathrm{MHz}, \mathrm{CDCl}_{3}\right) \delta 13.0,37.6,39.0$ $\left(\mathrm{t},{ }^{2} J_{\mathrm{CF}}=27.4 \mathrm{~Hz}\right), 40.7\left(\mathrm{t},{ }^{4} J_{\mathrm{CF}}=4.6 \mathrm{~Hz}\right), 100.4\left(\mathrm{t},{ }^{3} J_{\mathrm{CF}}=8.6 \mathrm{~Hz}\right), 117.5\left(\mathrm{t},{ }^{1} J_{\mathrm{CF}}=243.3 \mathrm{~Hz}\right), 125.1$, $125.2\left(\mathrm{t},{ }^{3} J_{\mathrm{CF}}=6.3 \mathrm{~Hz}\right), 147.8\left(\mathrm{t},{ }^{2} J_{\mathrm{CF}}=28.6 \mathrm{~Hz}\right), 151.4,161.9 ;{ }^{19} \mathrm{~F}$ NMR $\left(282.4 \mathrm{MHz}, \mathrm{CDCl}_{3}\right) \delta-97.5$ (t, $J=14.6 \mathrm{~Hz}, 2 \mathrm{~F})$. IR (film): 1709, 1660, 1630, 1472, 1395, 1342, $1288 \mathrm{~cm}^{-1}$. HRMS (EI) calcd for $\mathrm{C}_{11} \mathrm{H}_{12} \mathrm{~F}_{2} \mathrm{~N}_{2} \mathrm{O}_{2}\left(\mathrm{M}^{+}\right): 242.0867$, found: 242.0823 . 


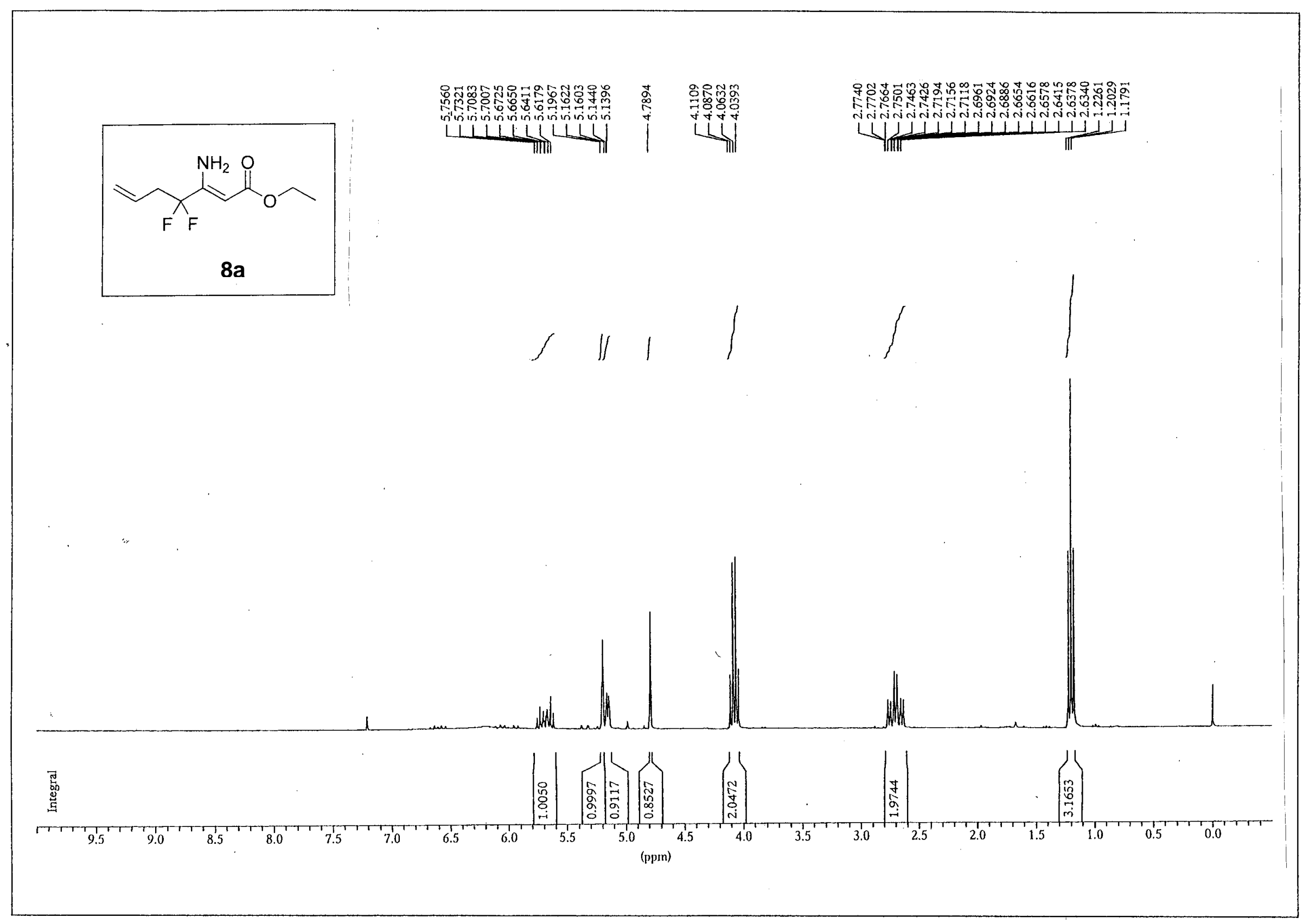




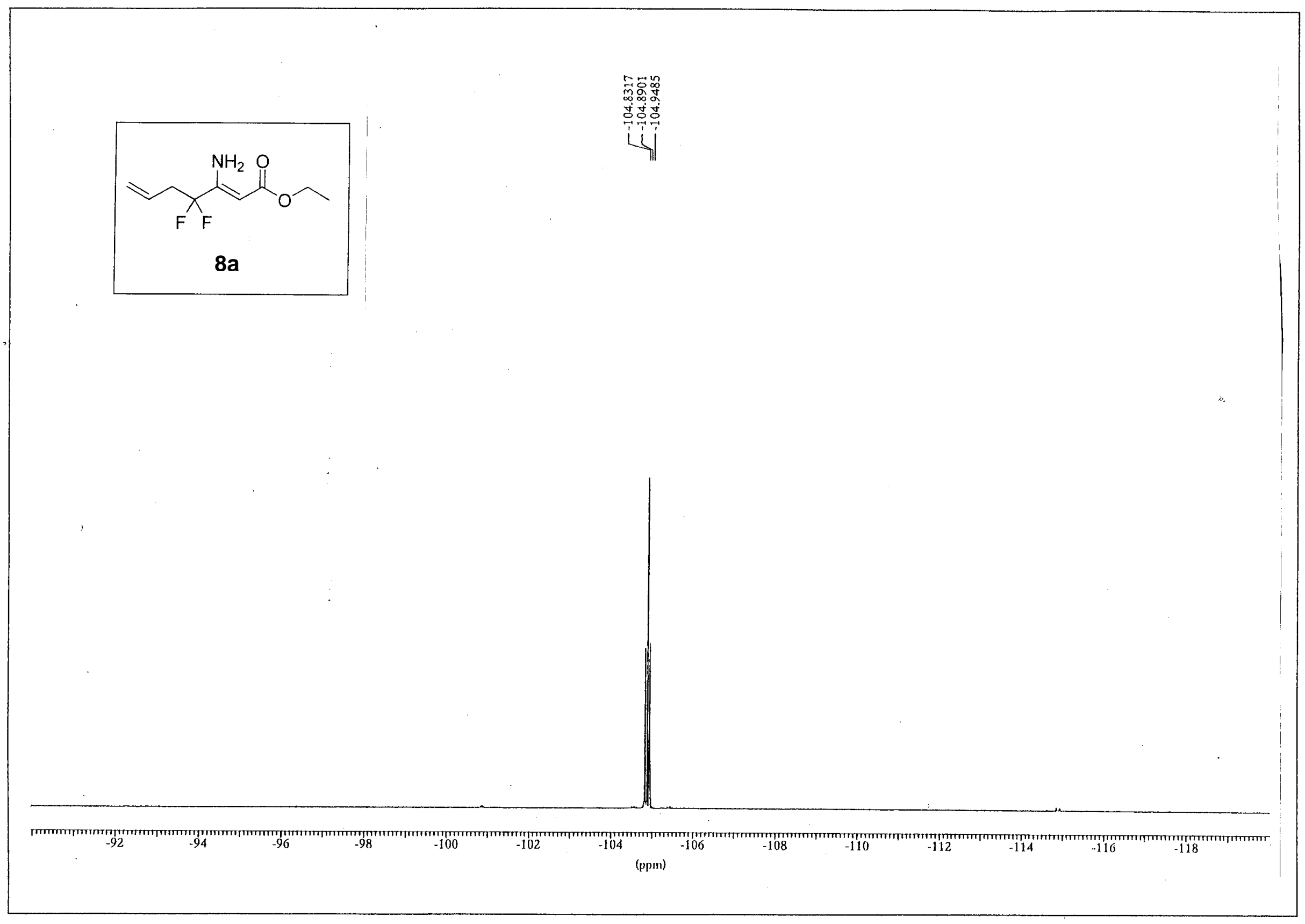




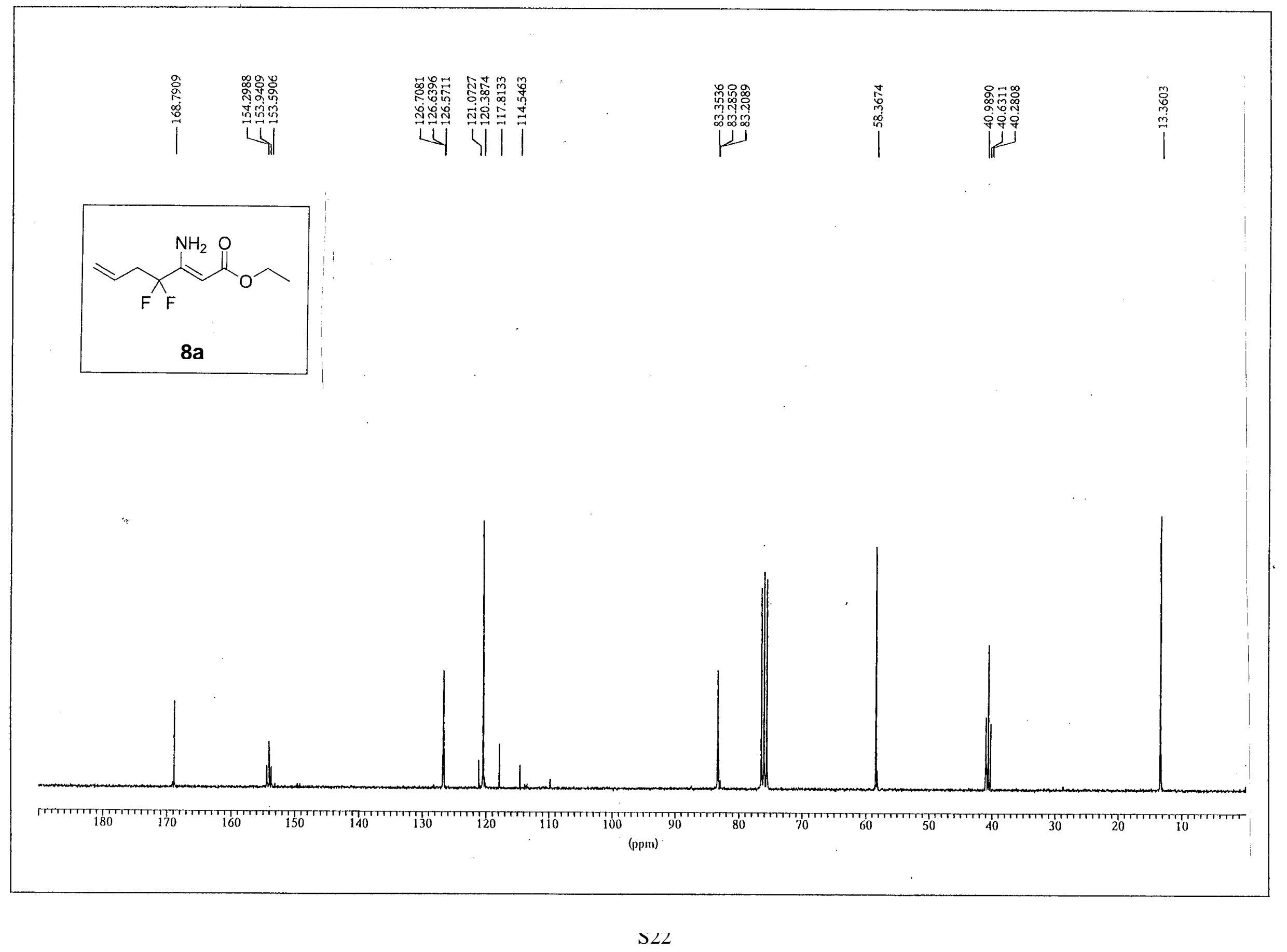




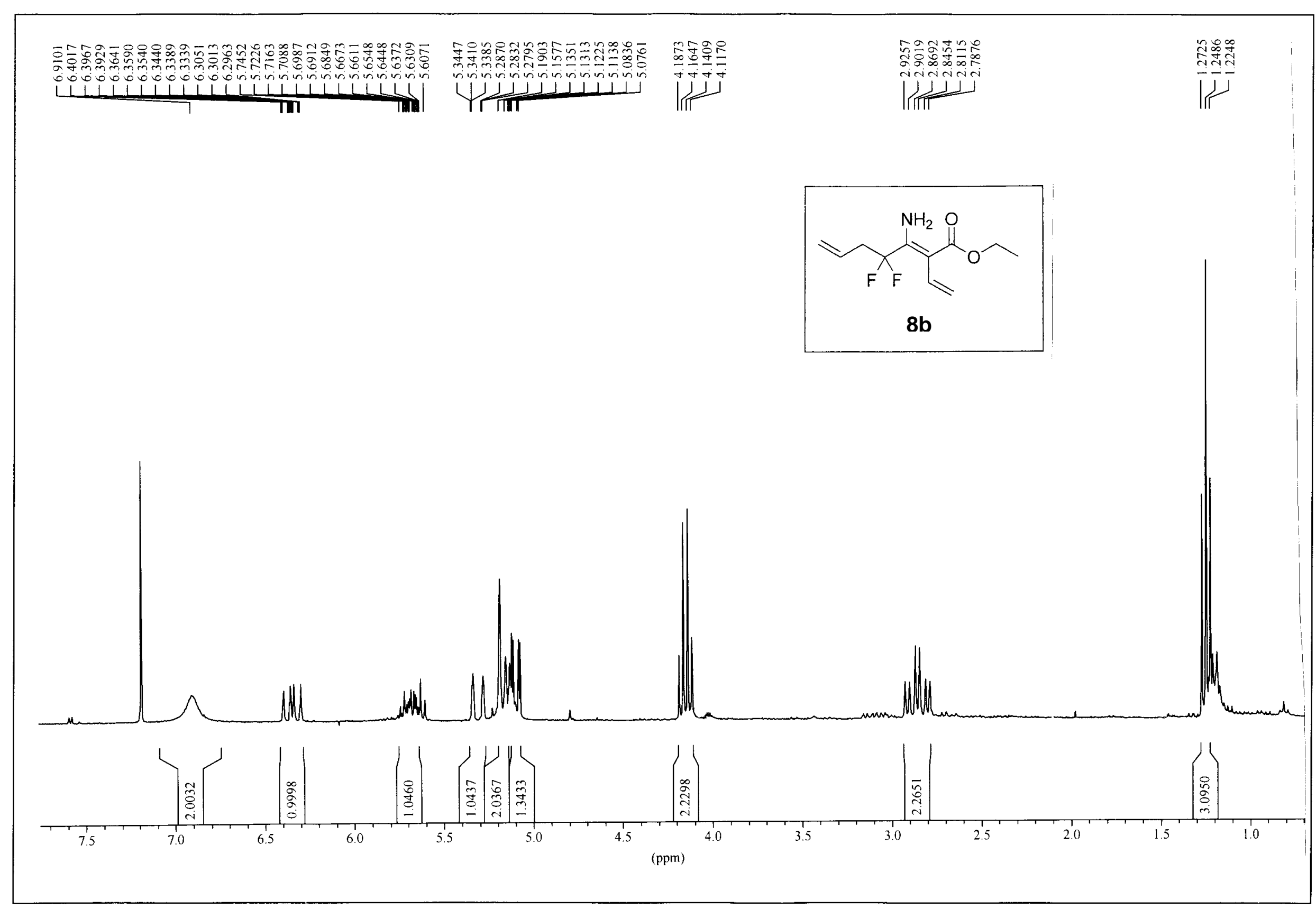




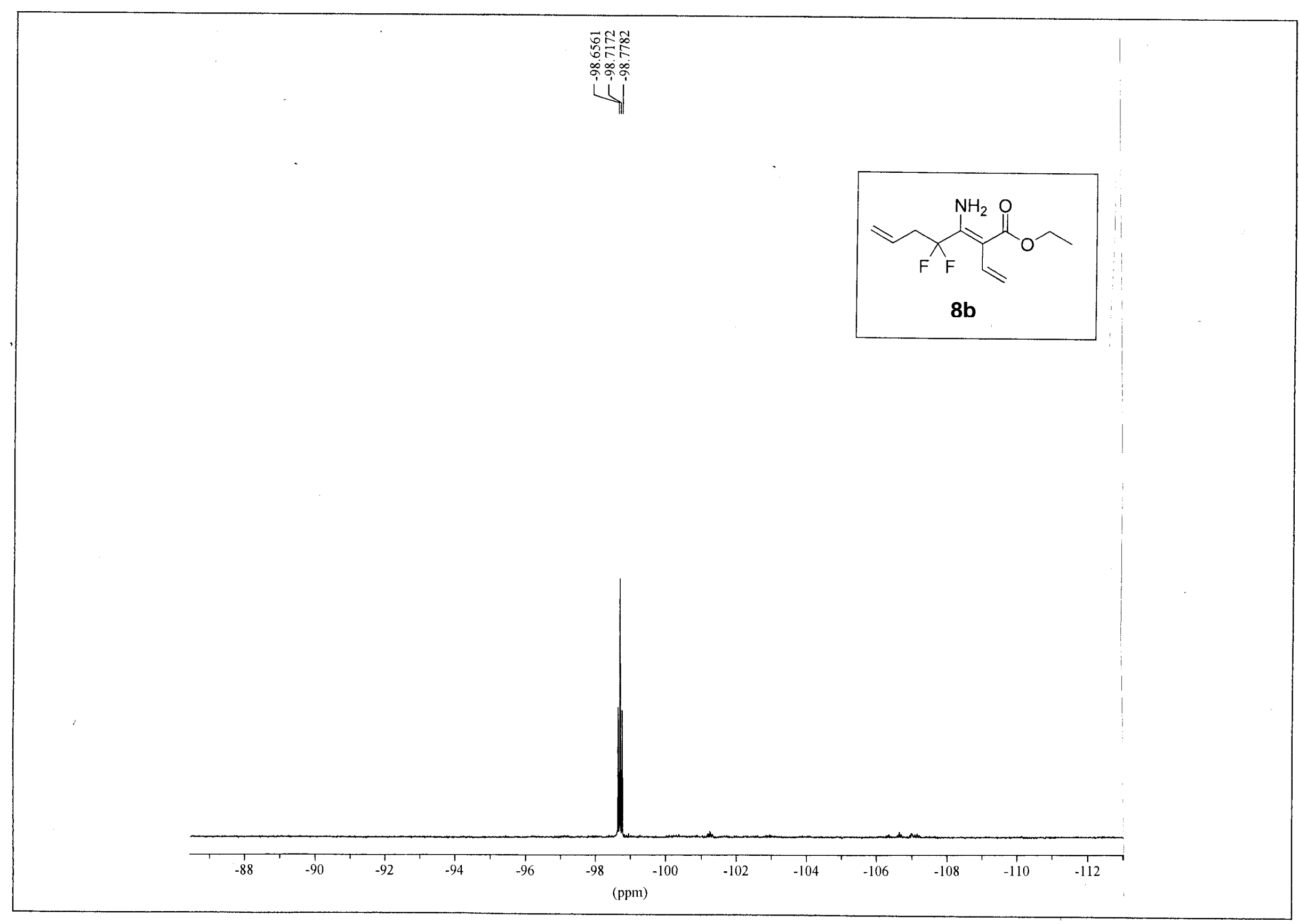




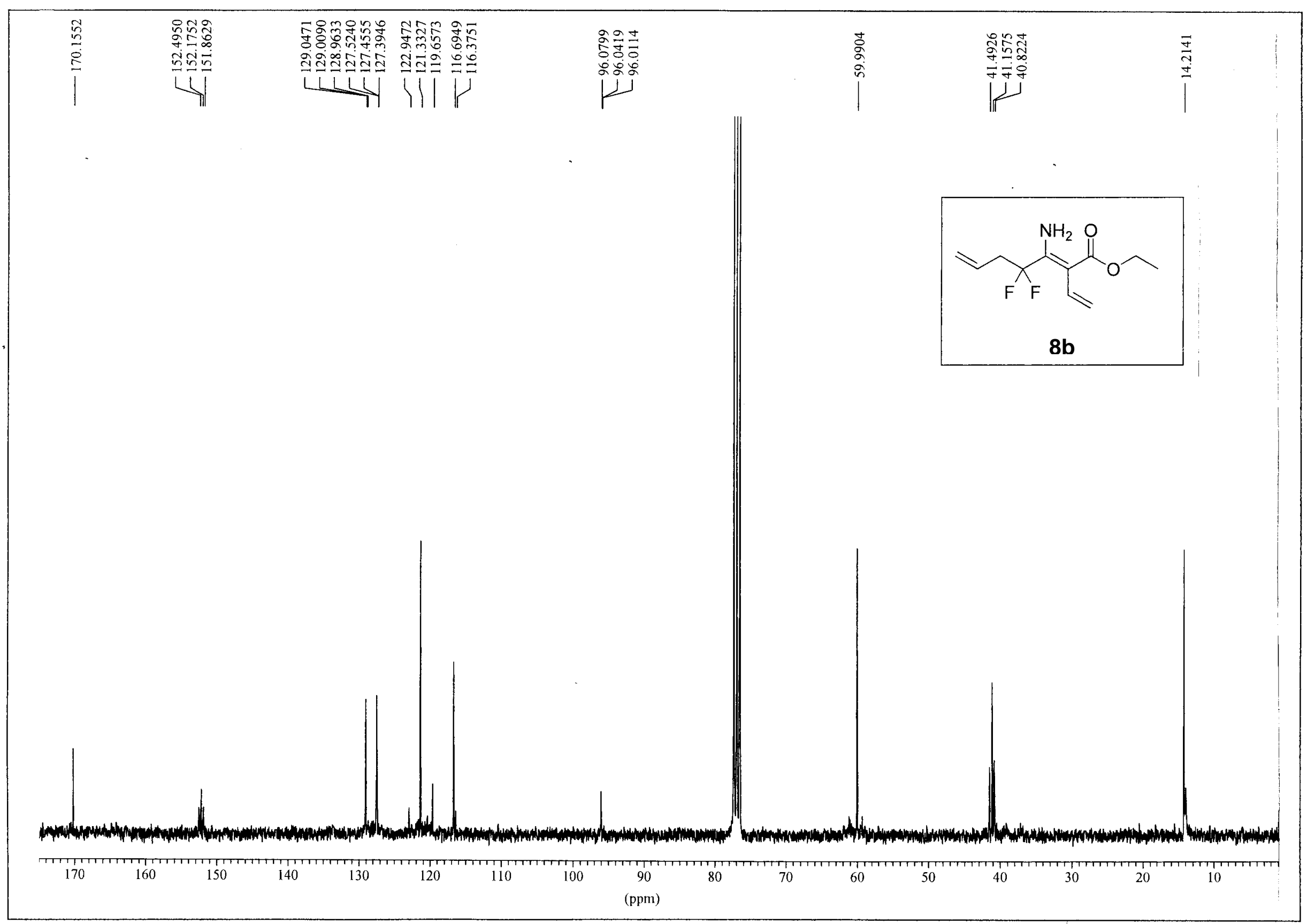




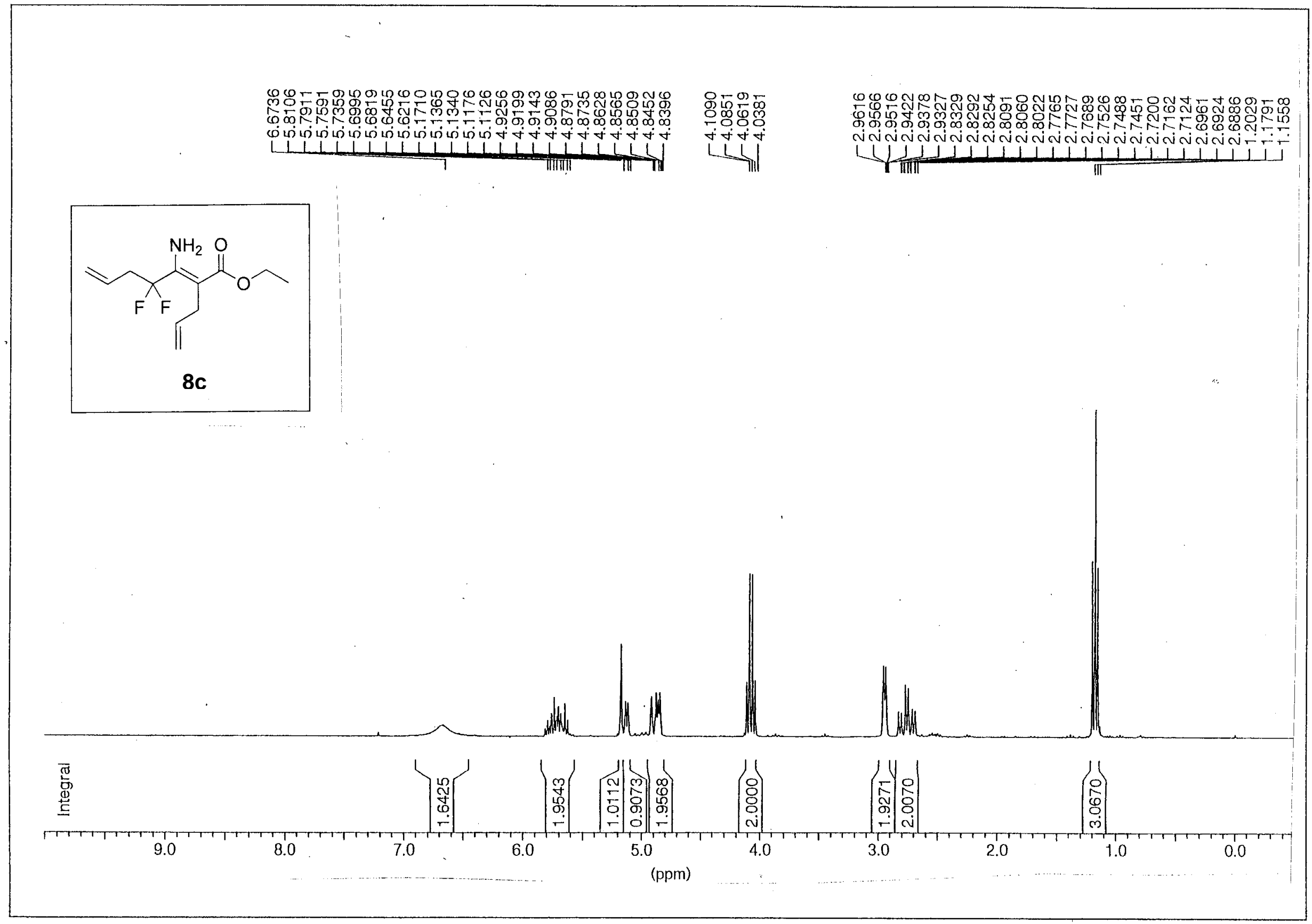




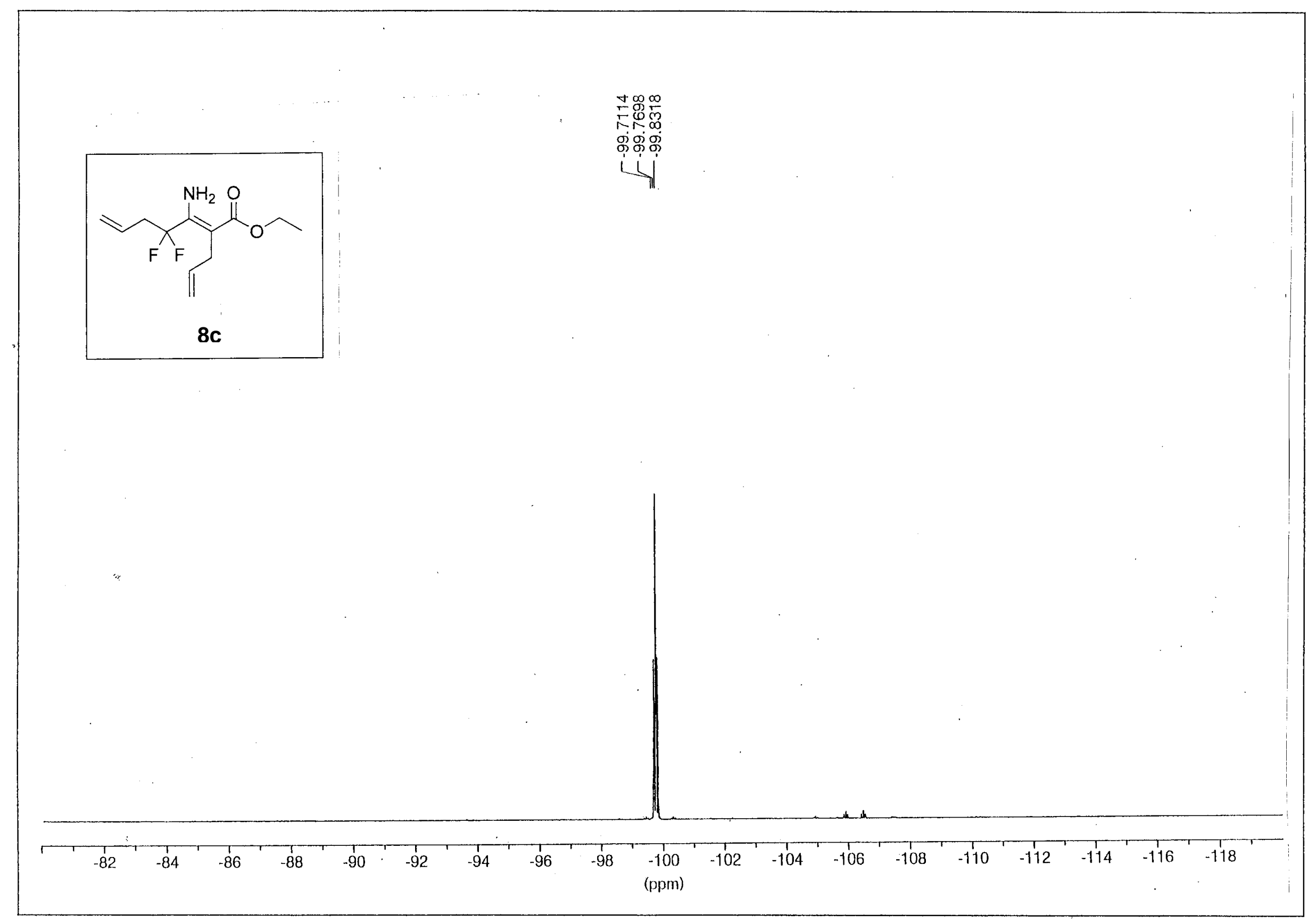




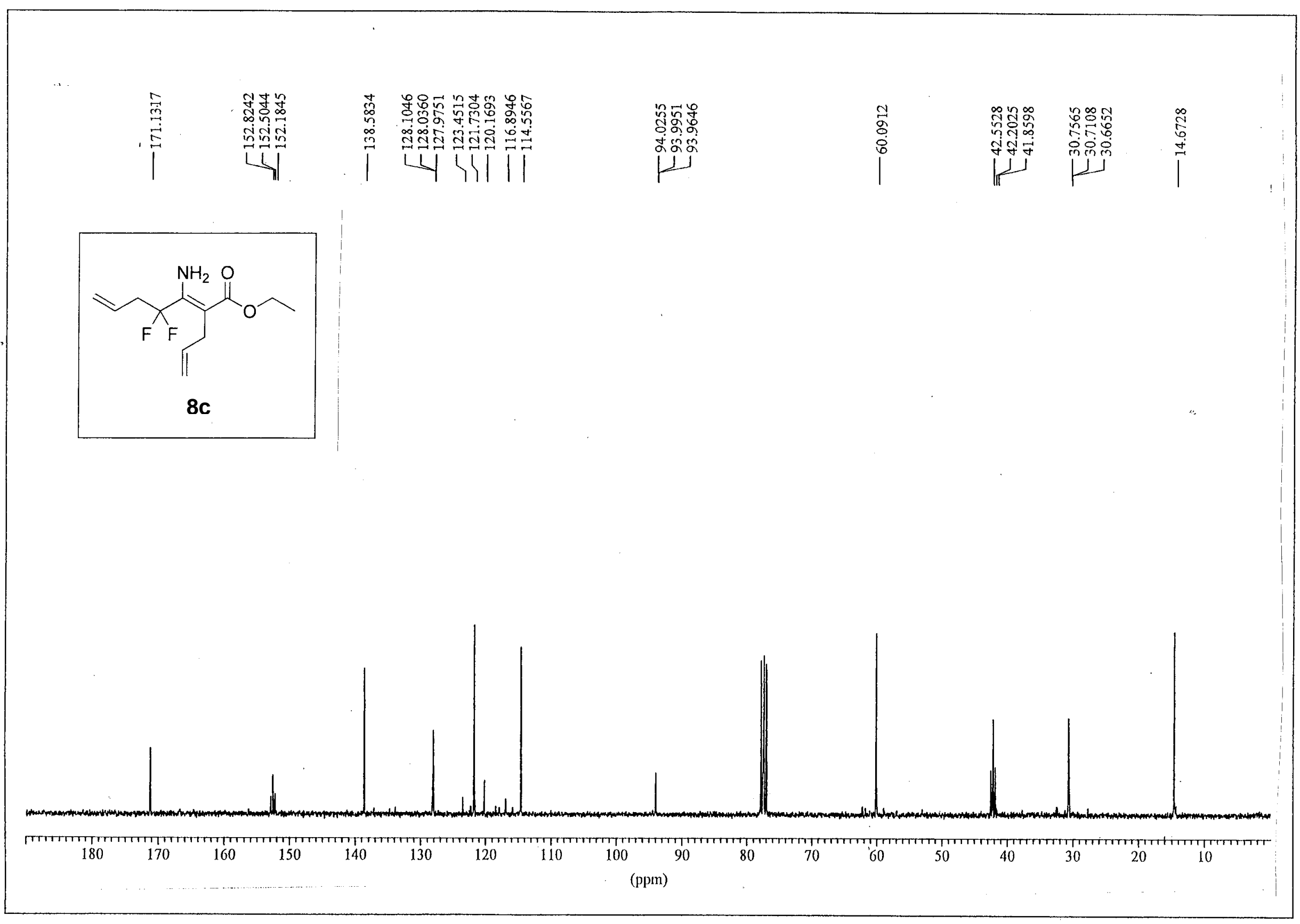




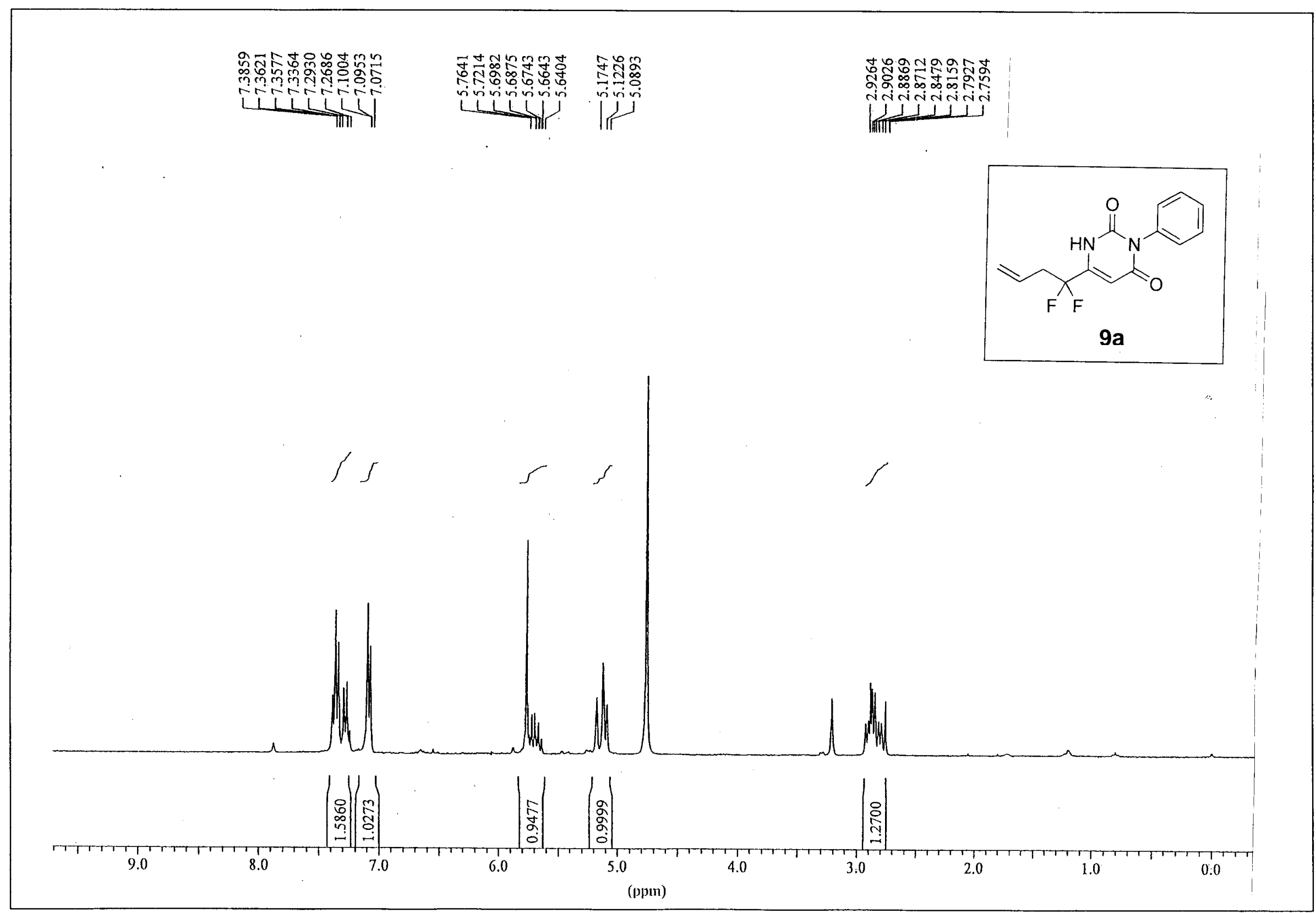




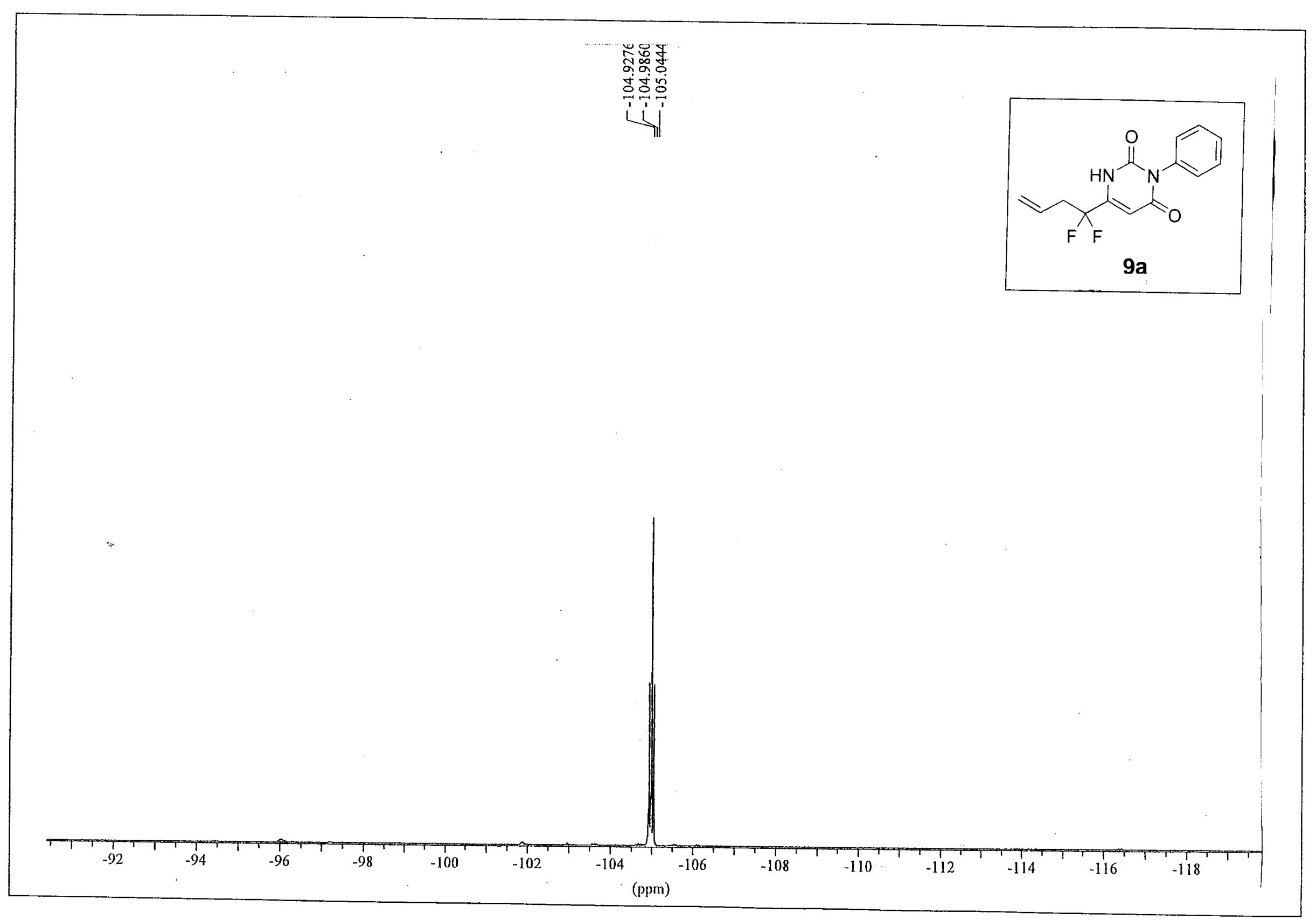




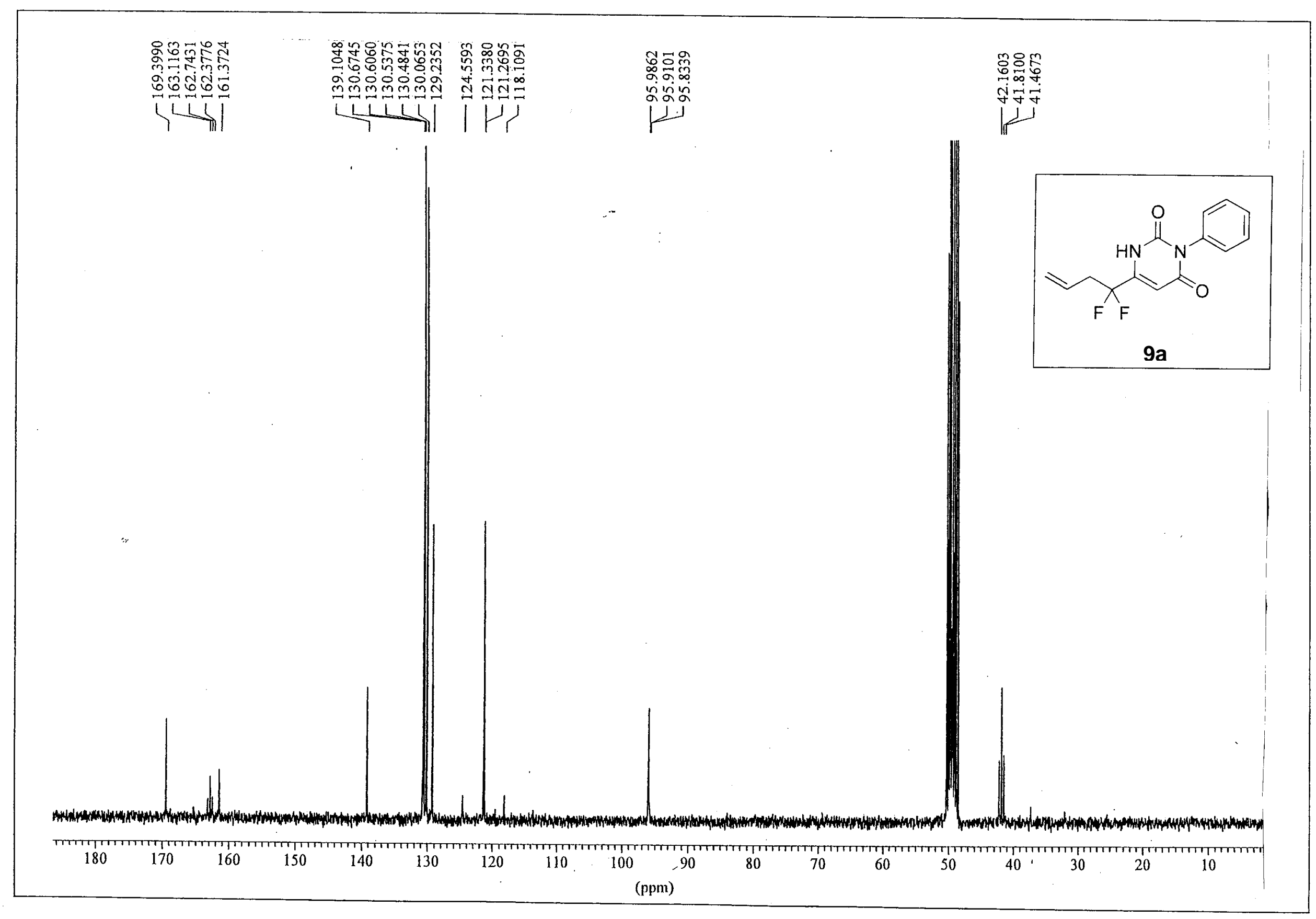




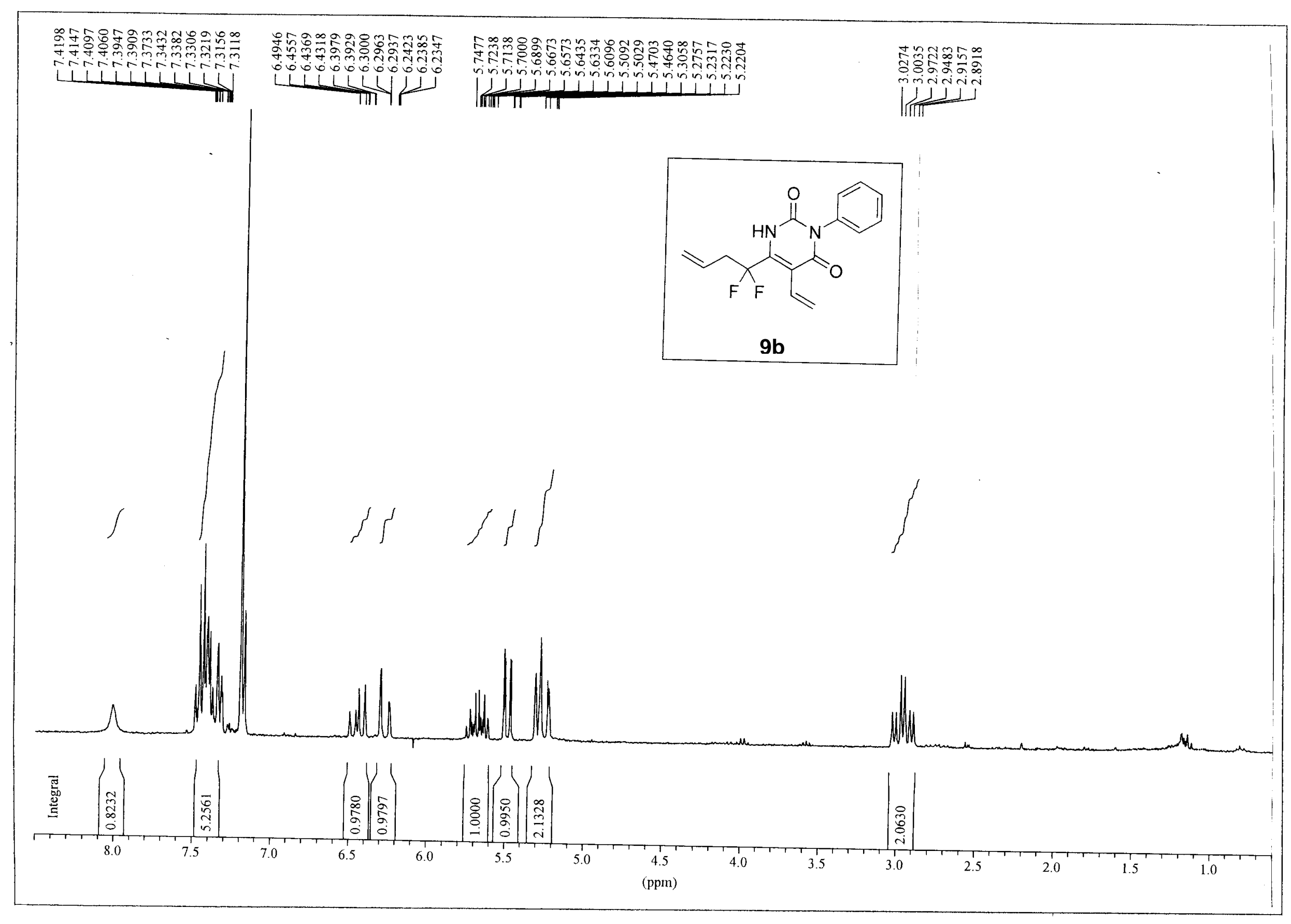




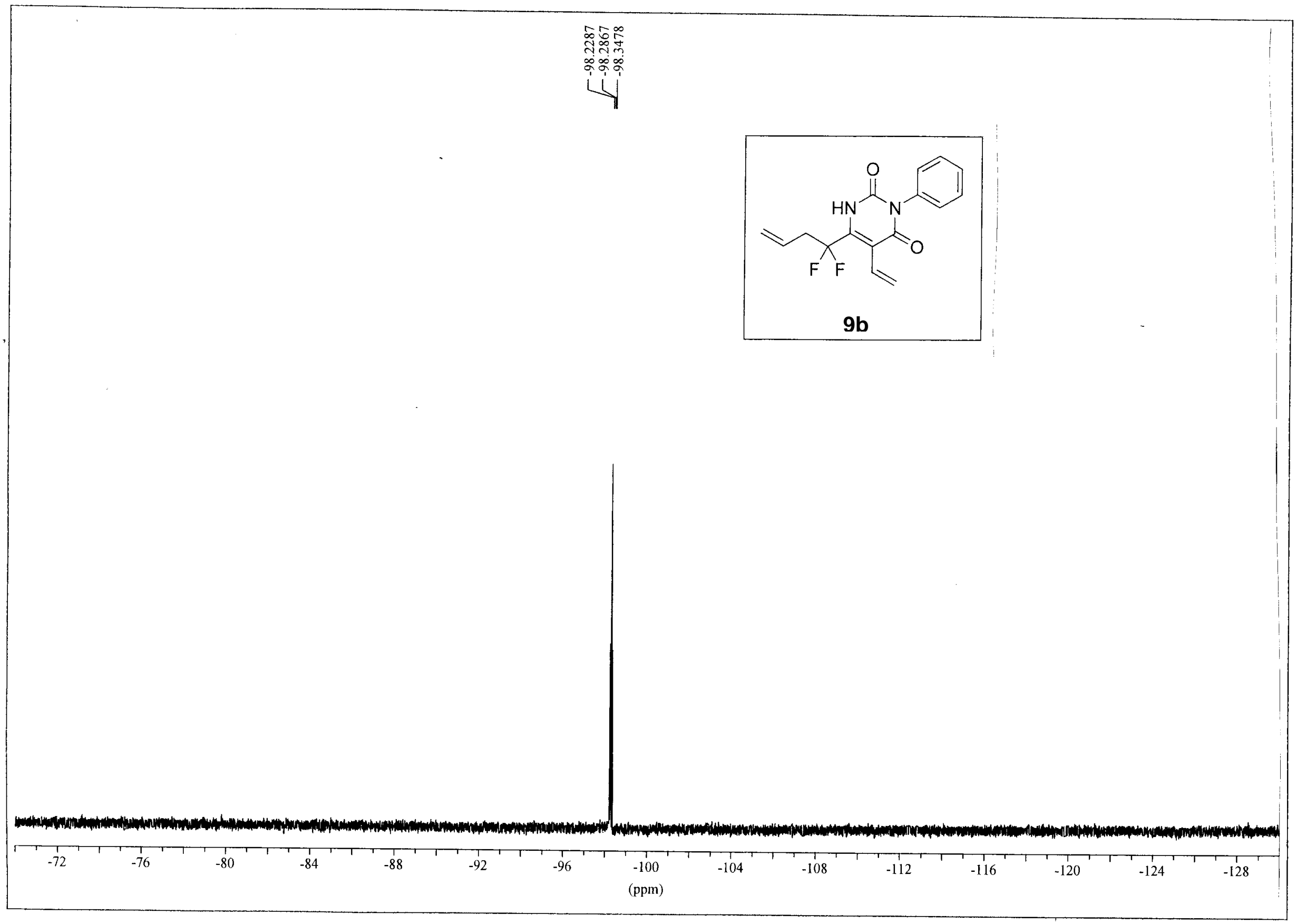




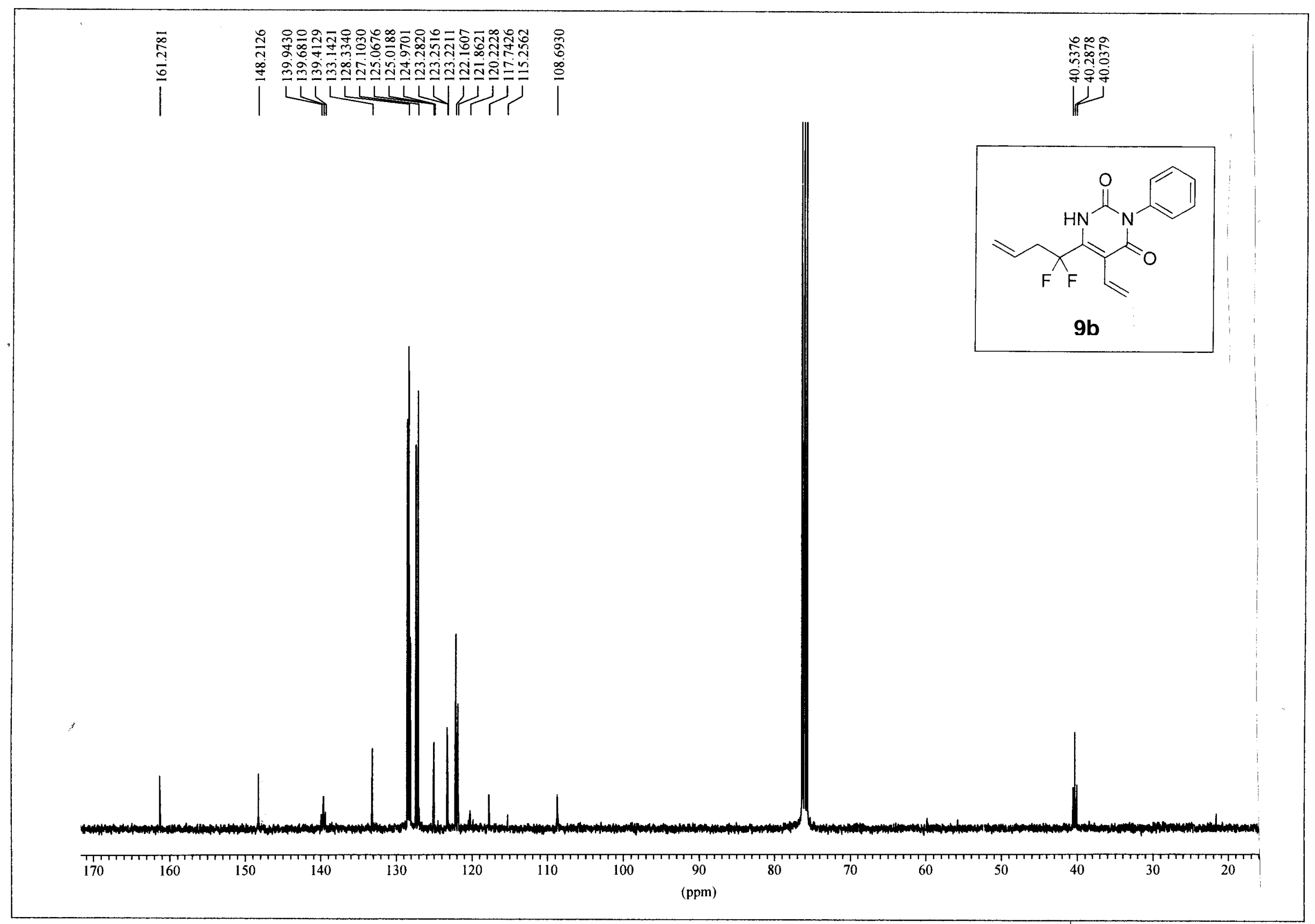




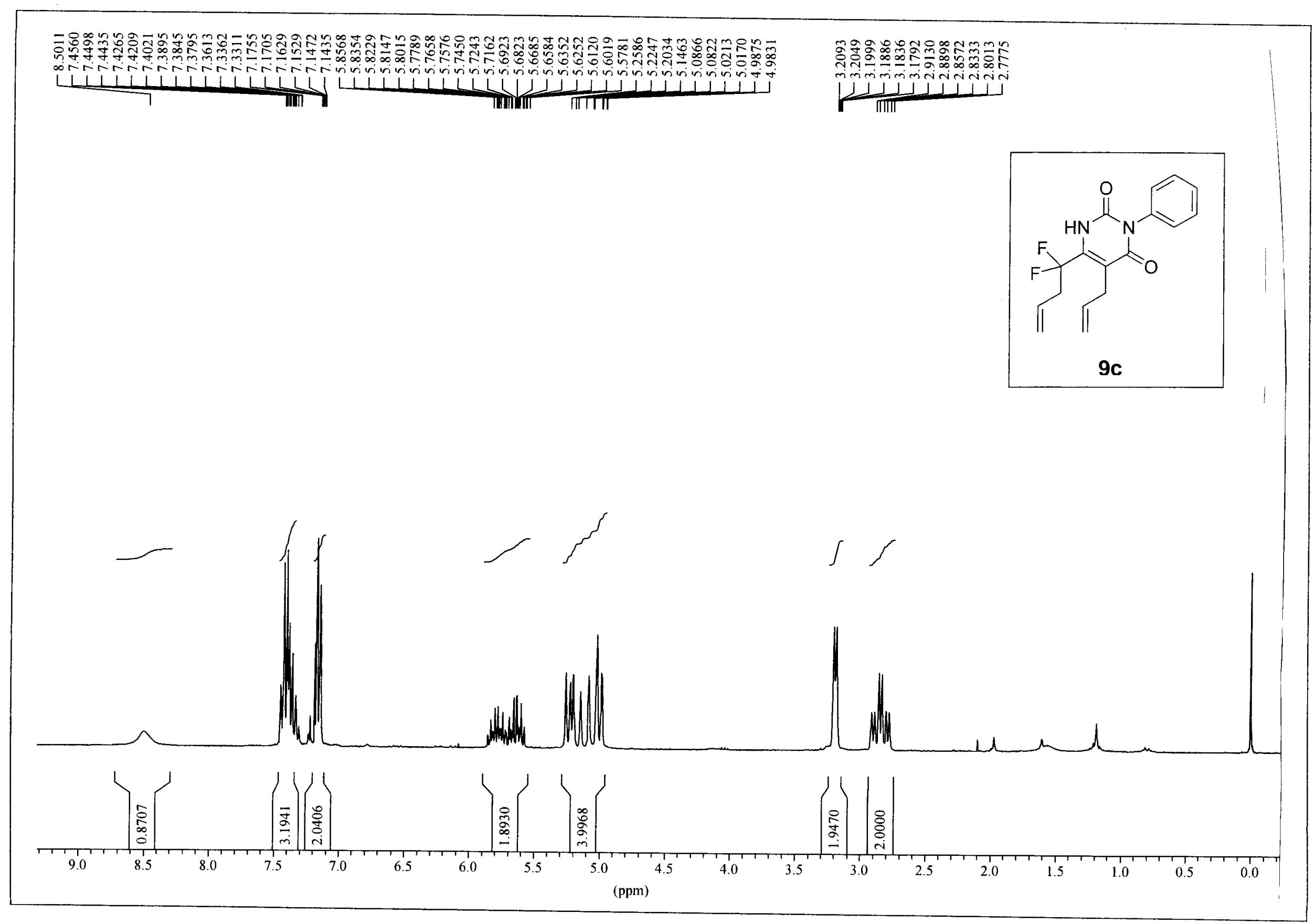




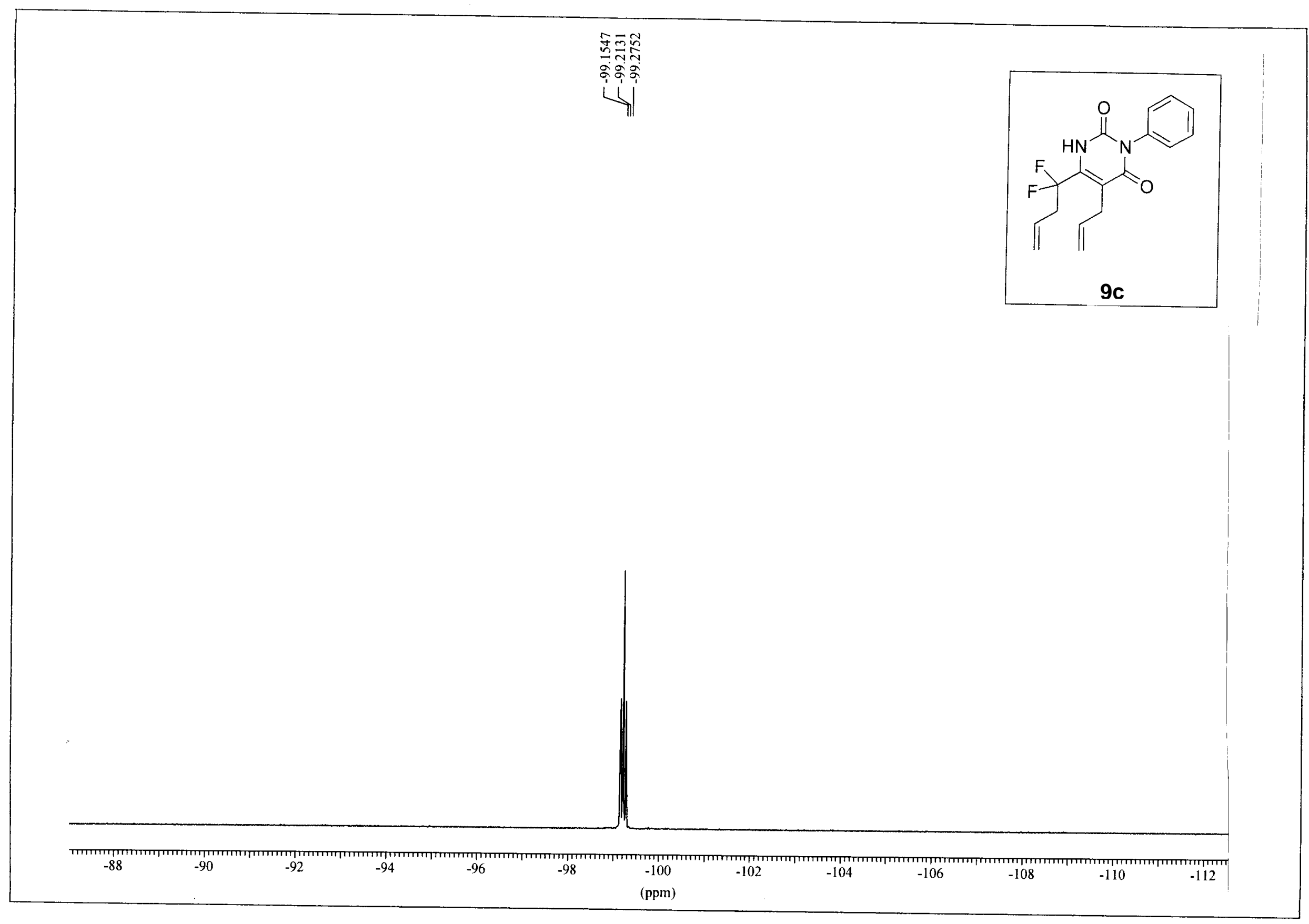




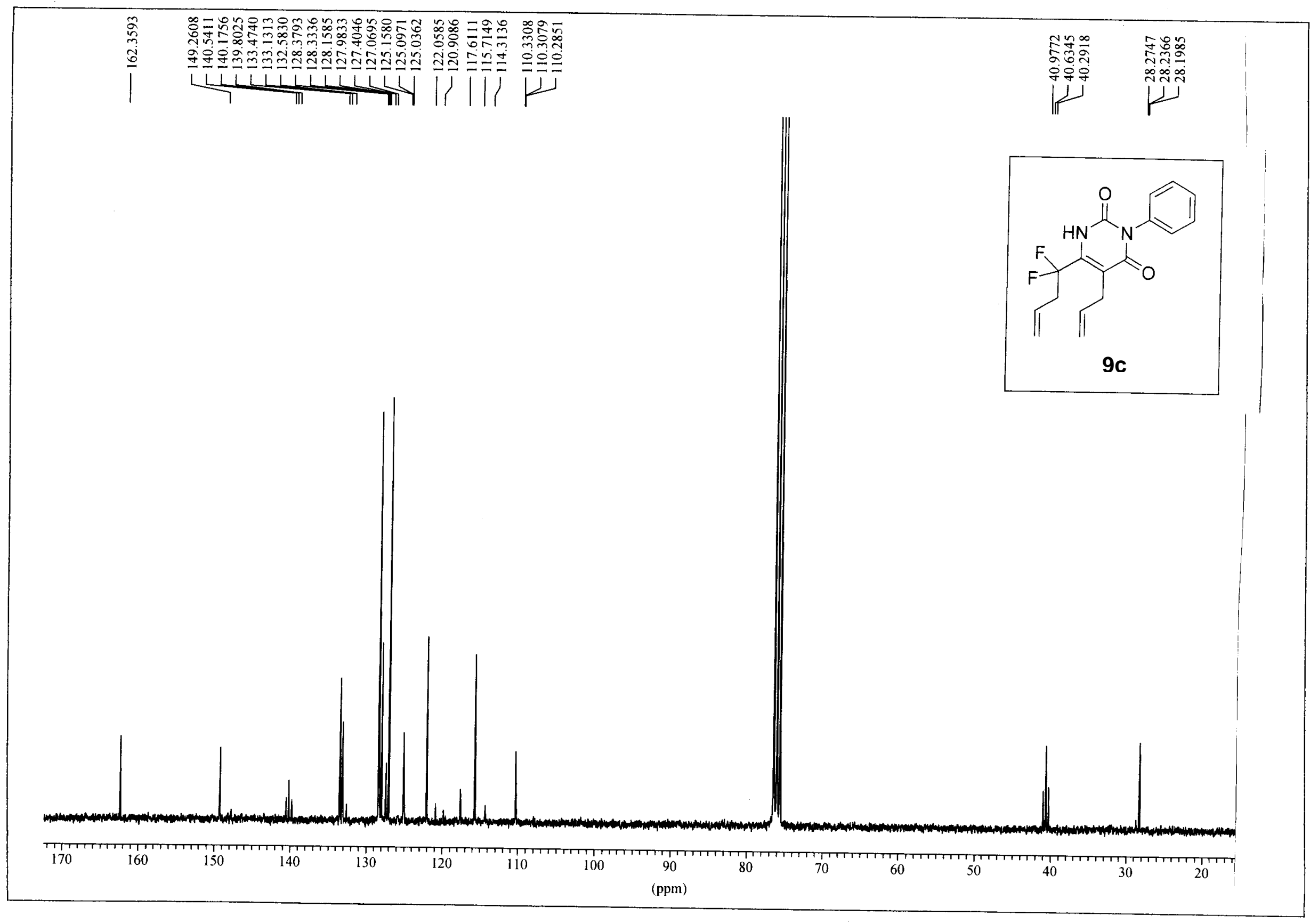




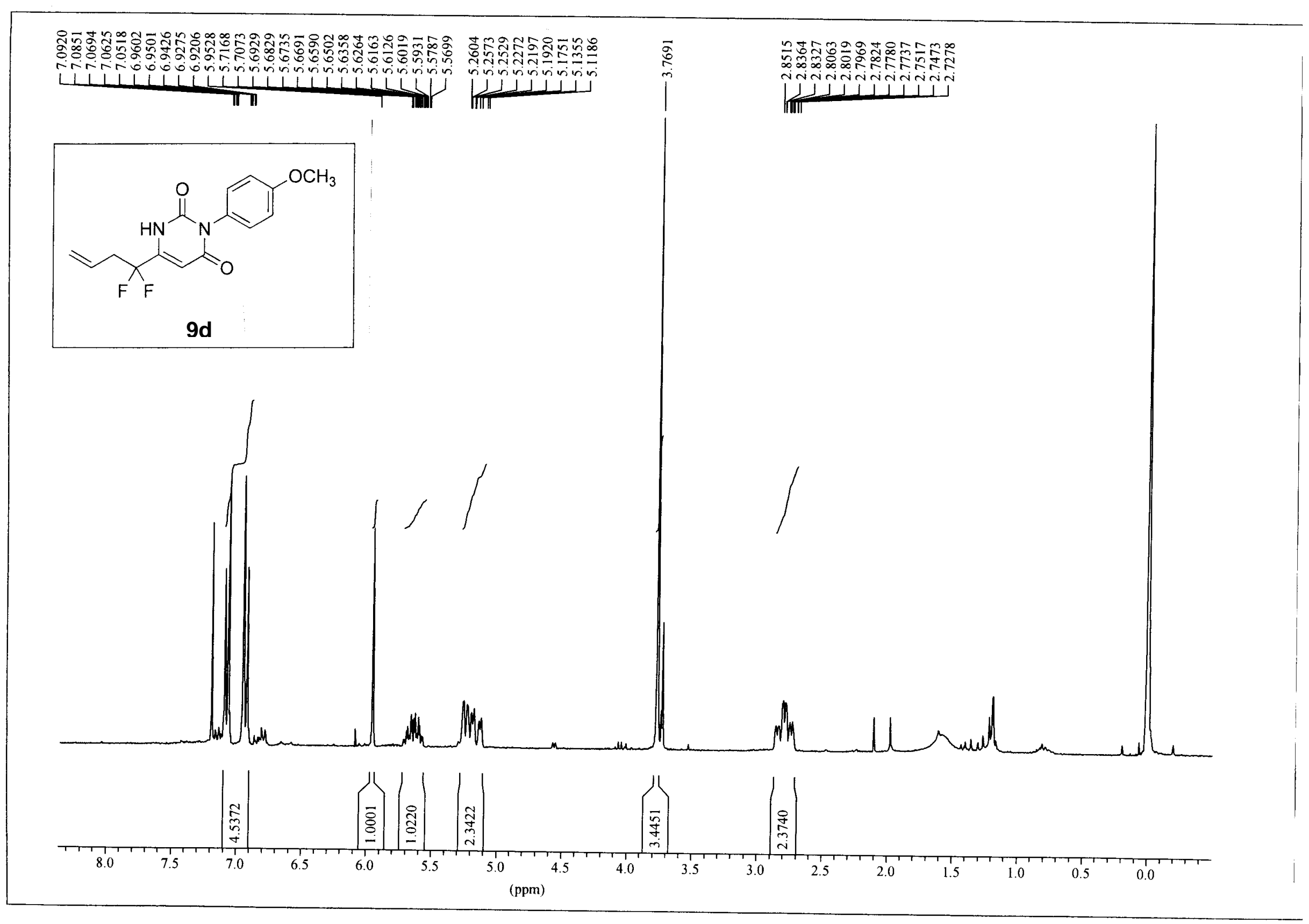



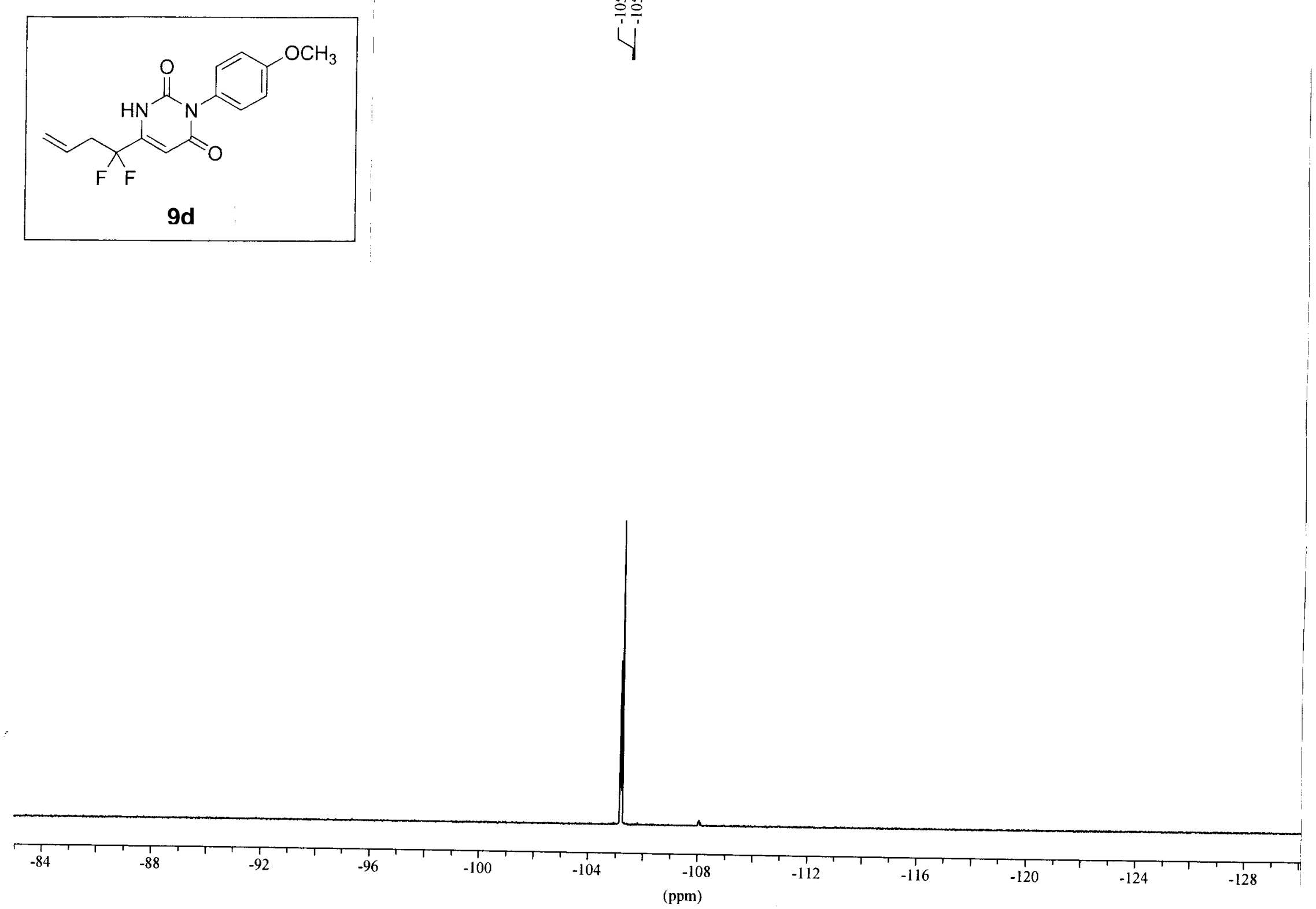


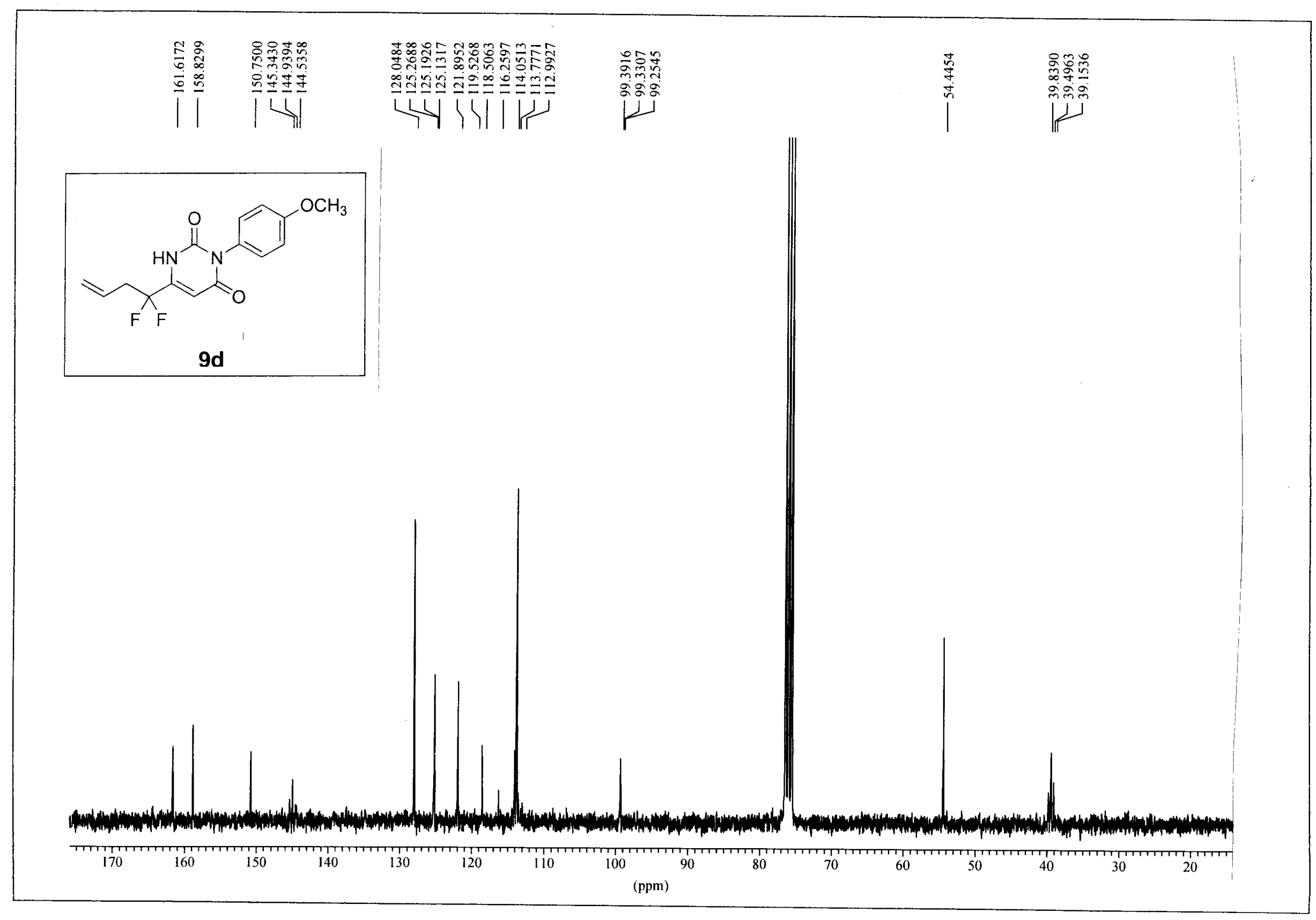




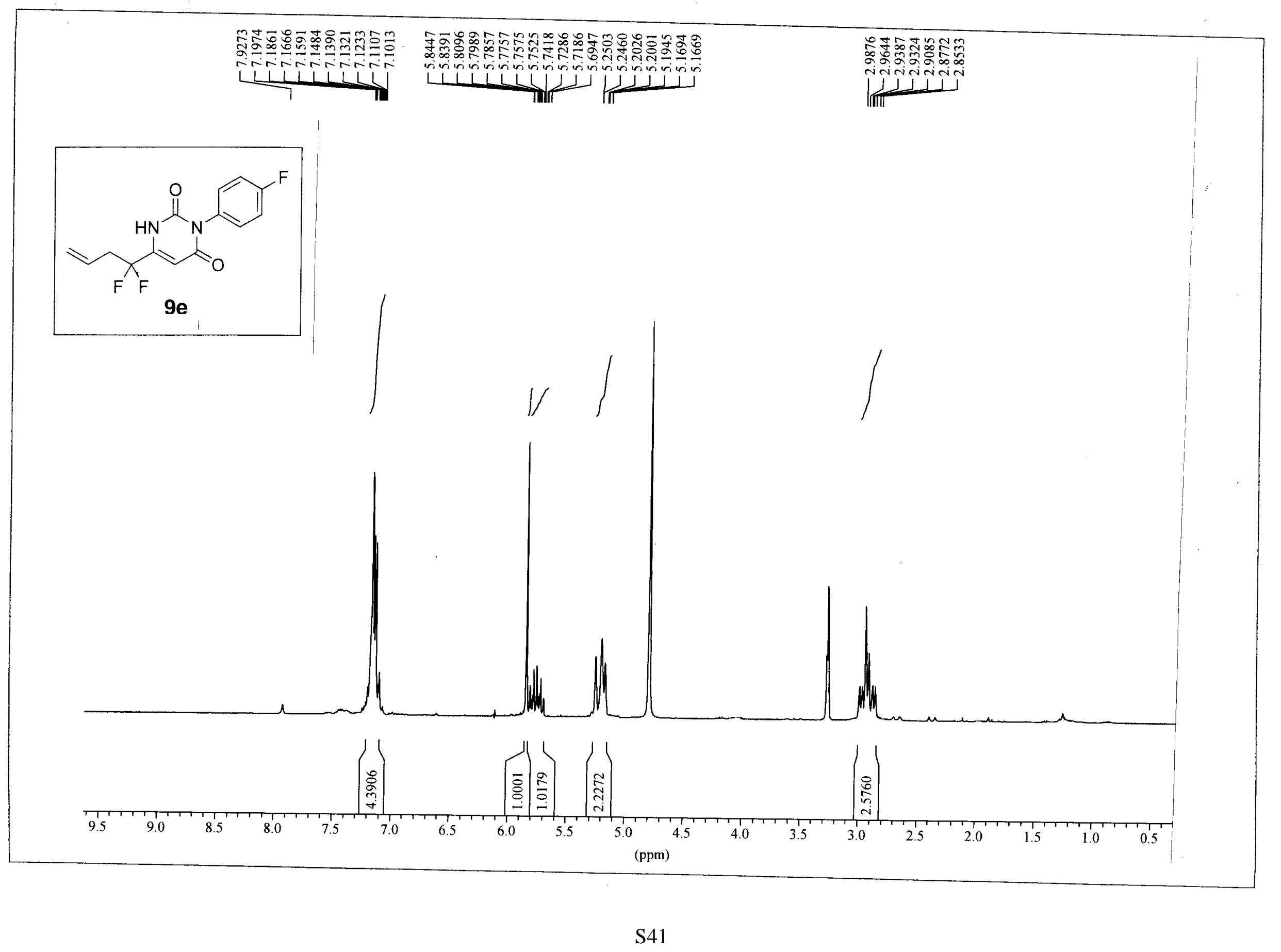




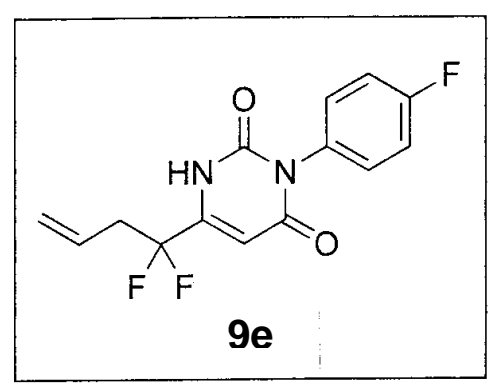

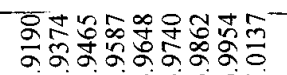

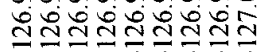

ن

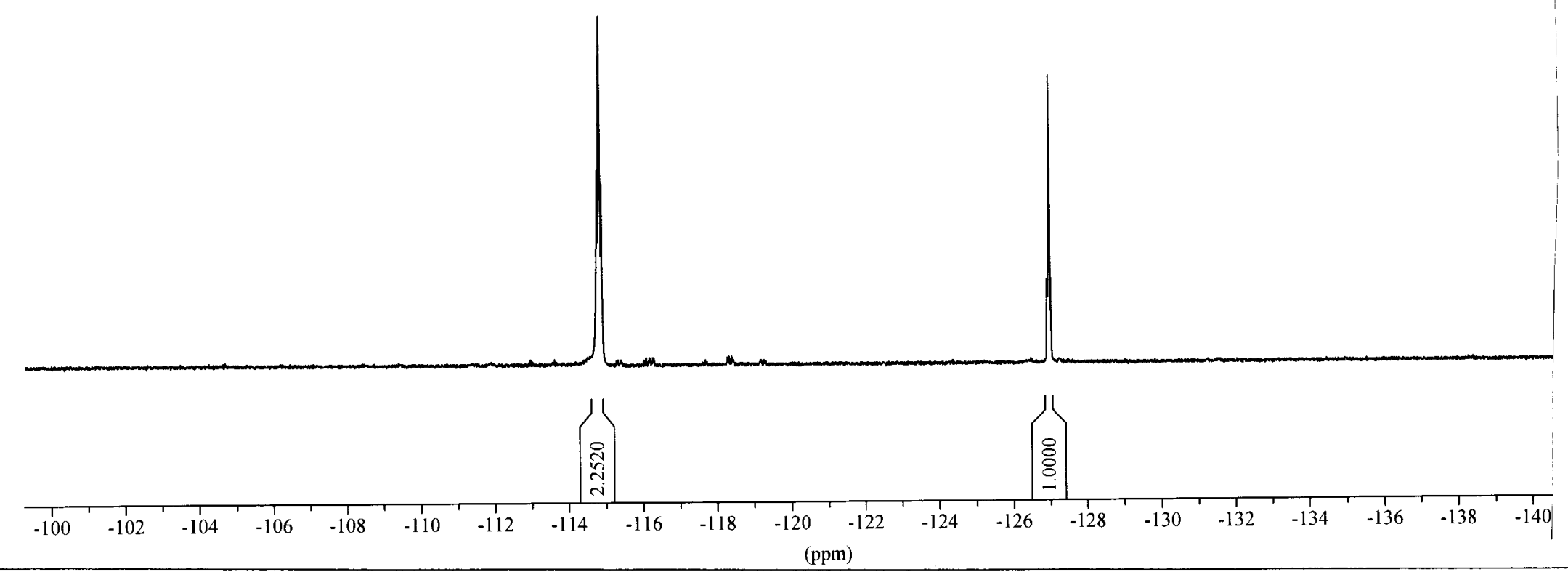




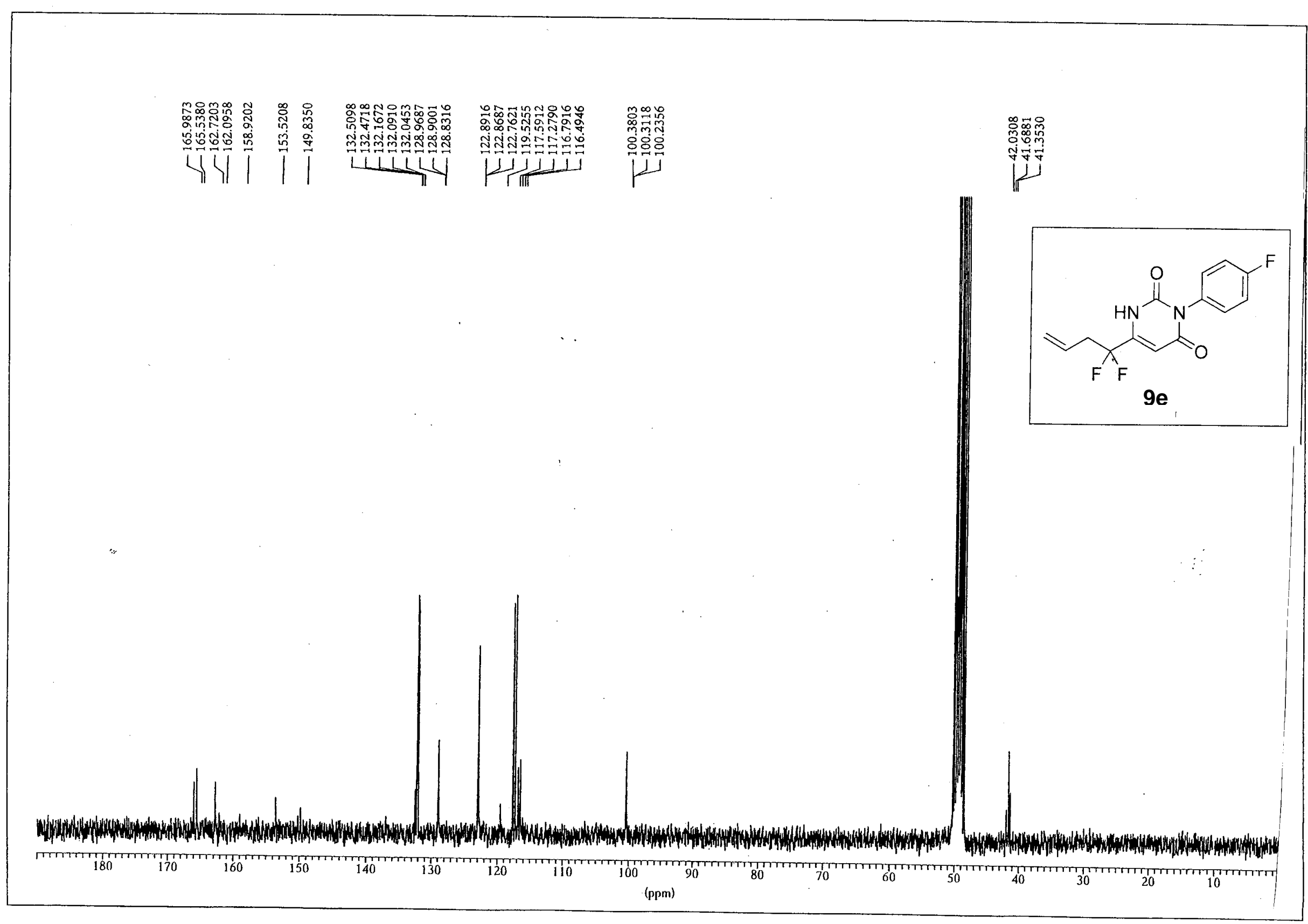




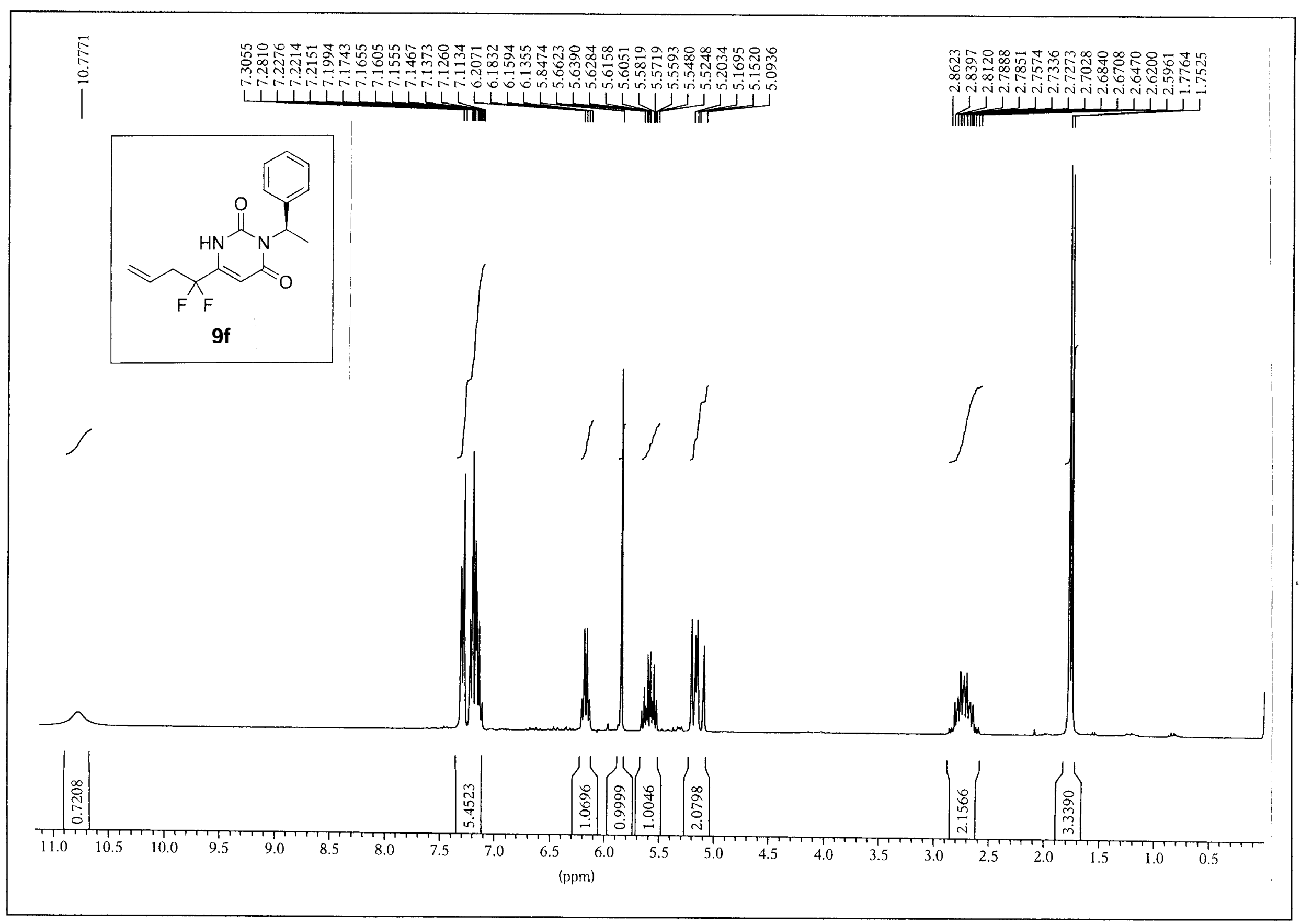




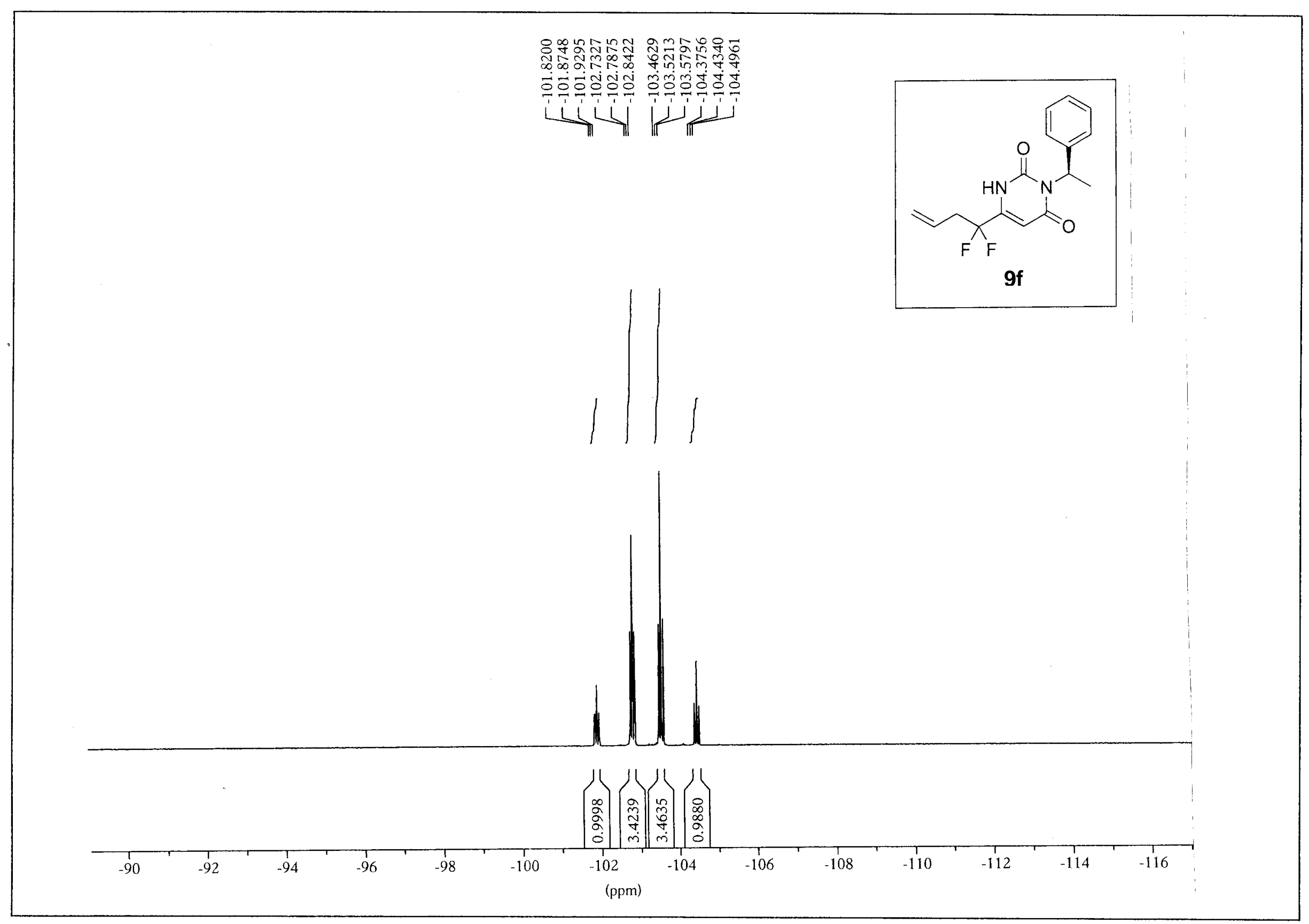




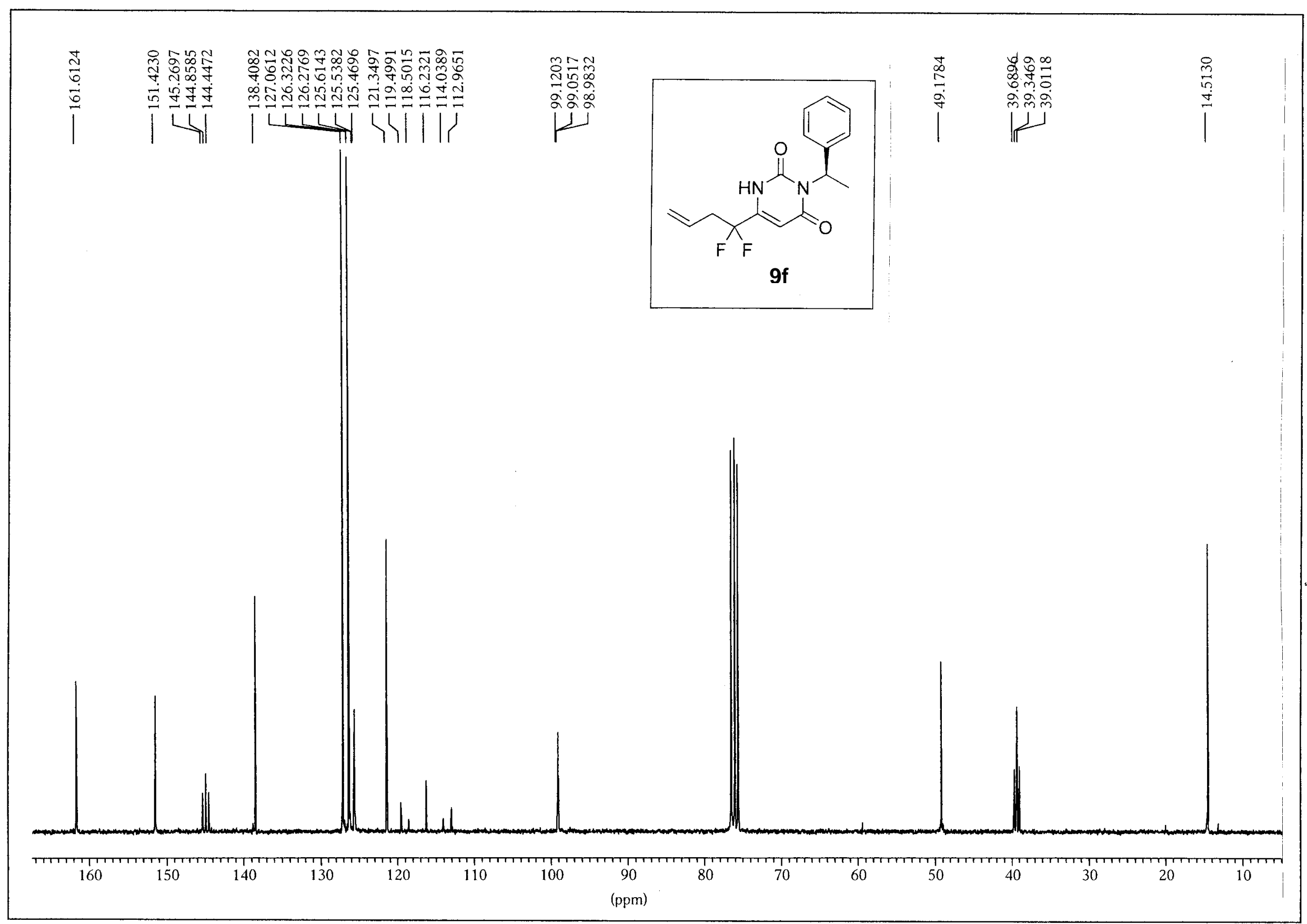




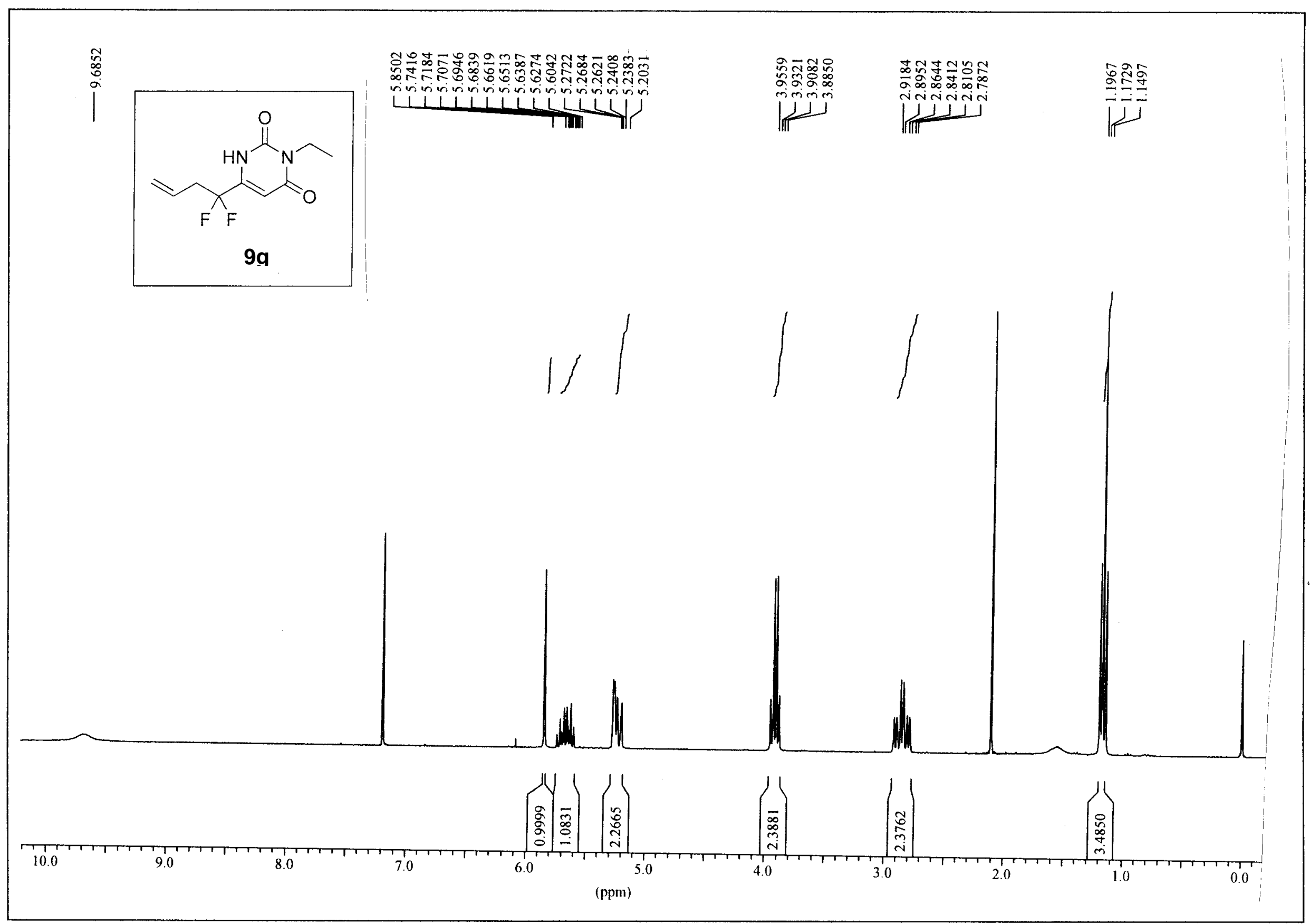




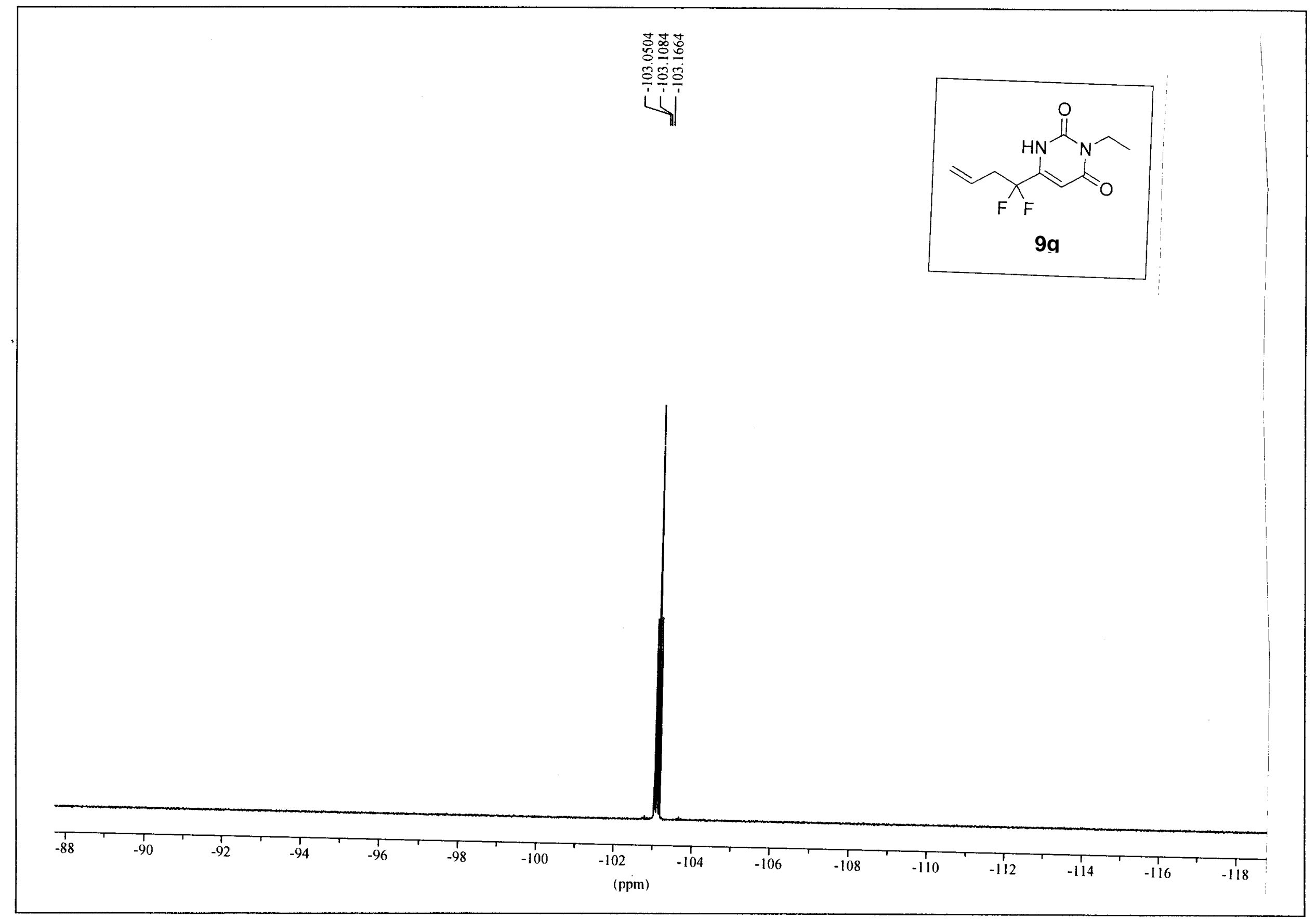




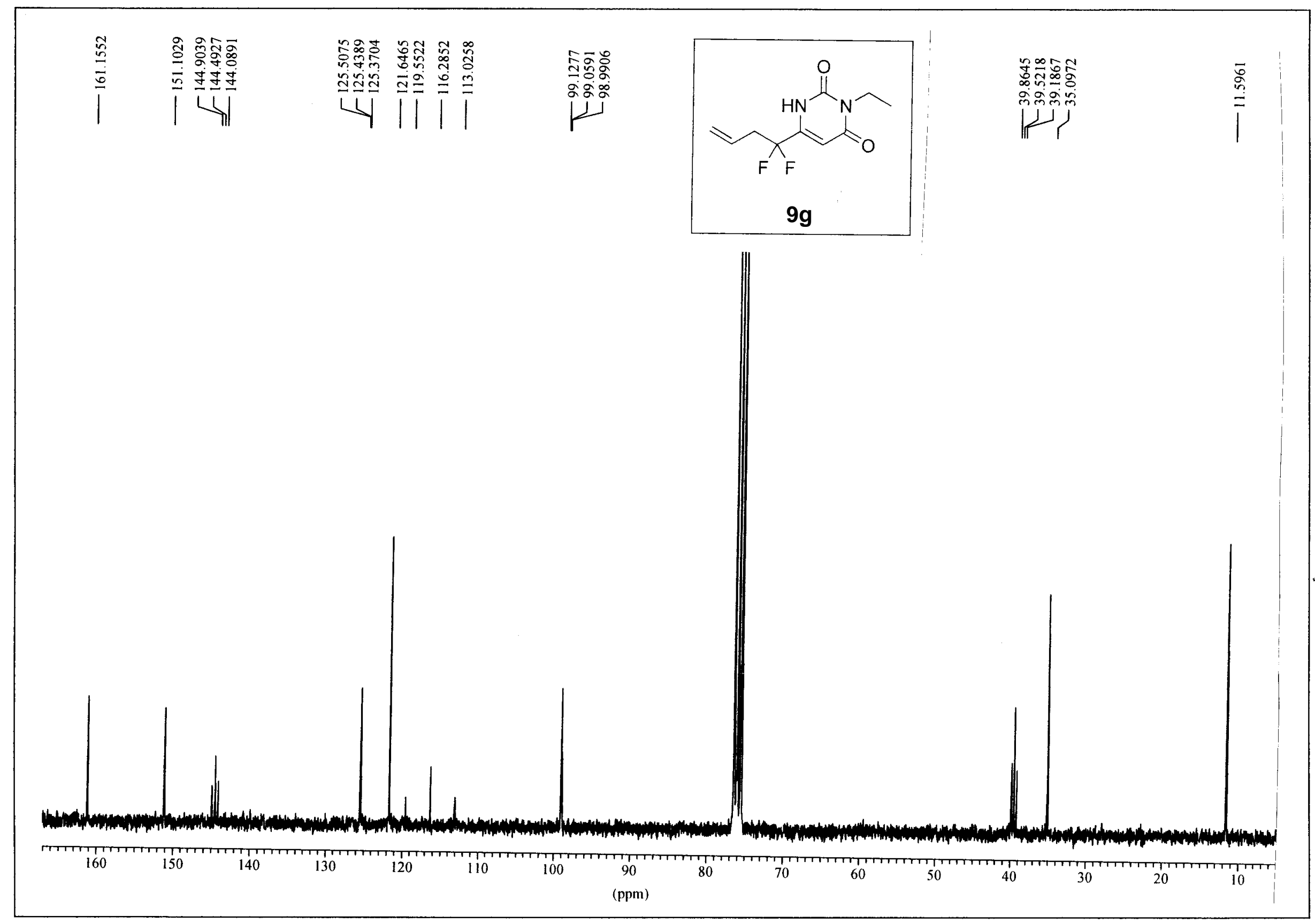




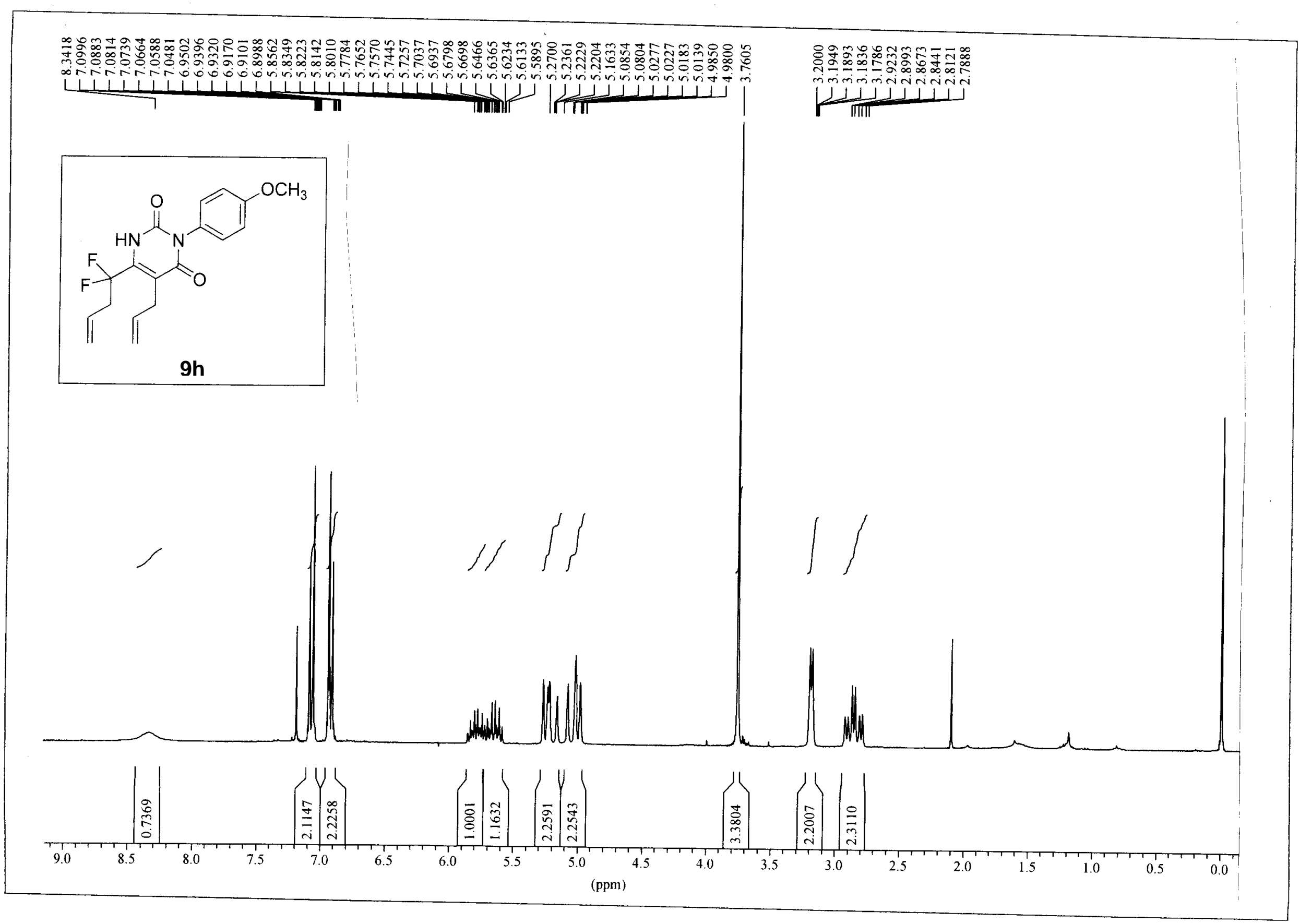




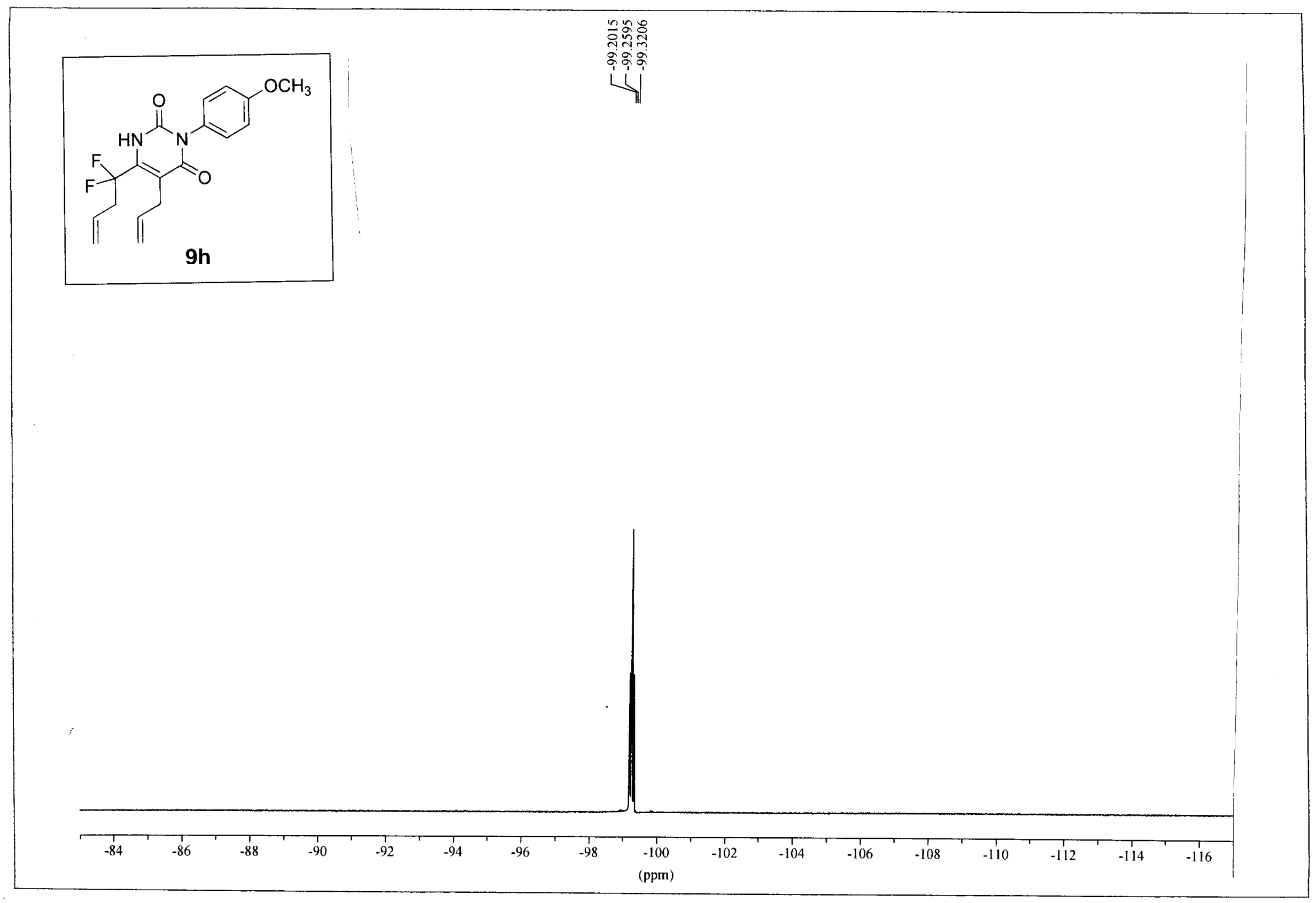




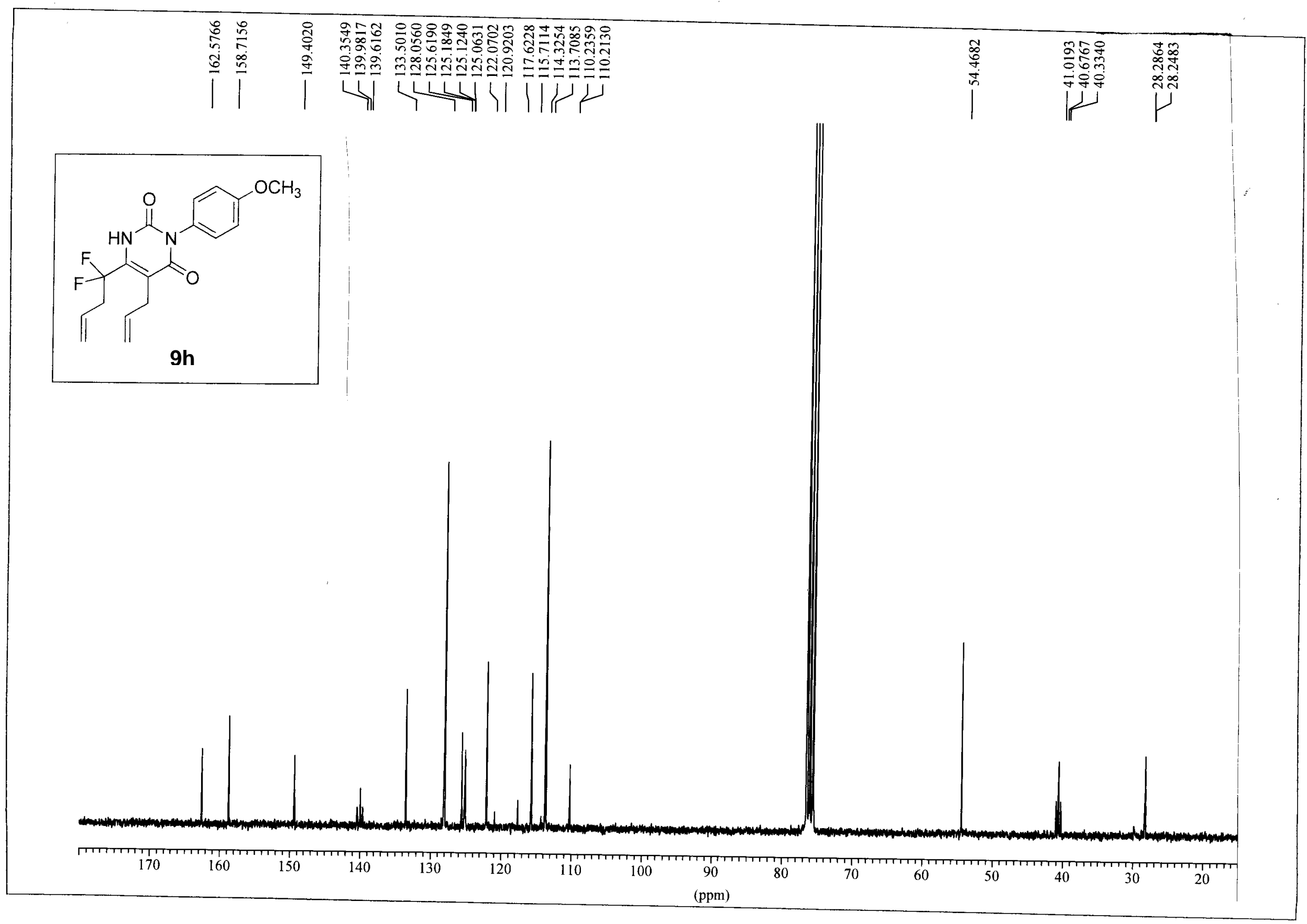




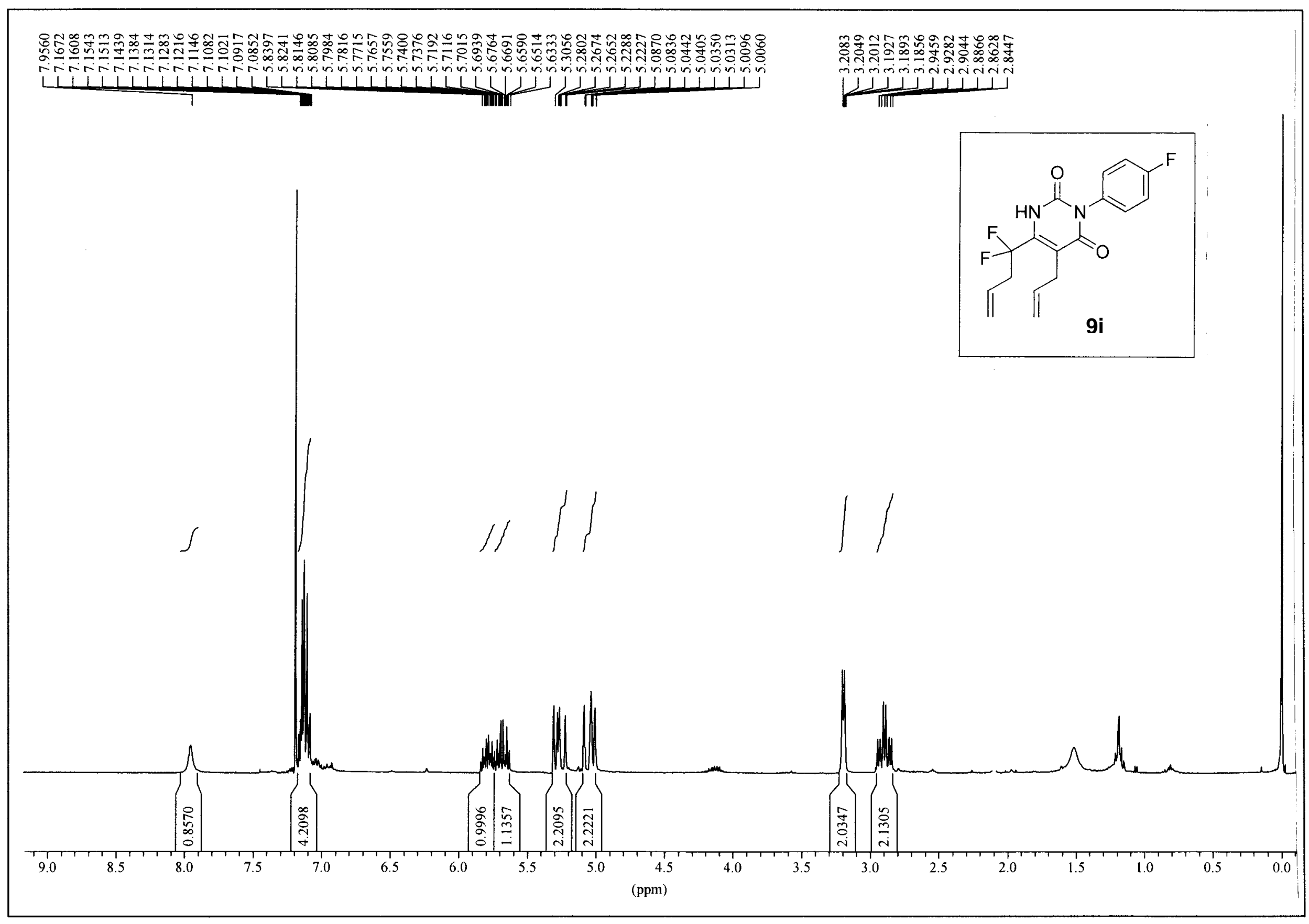




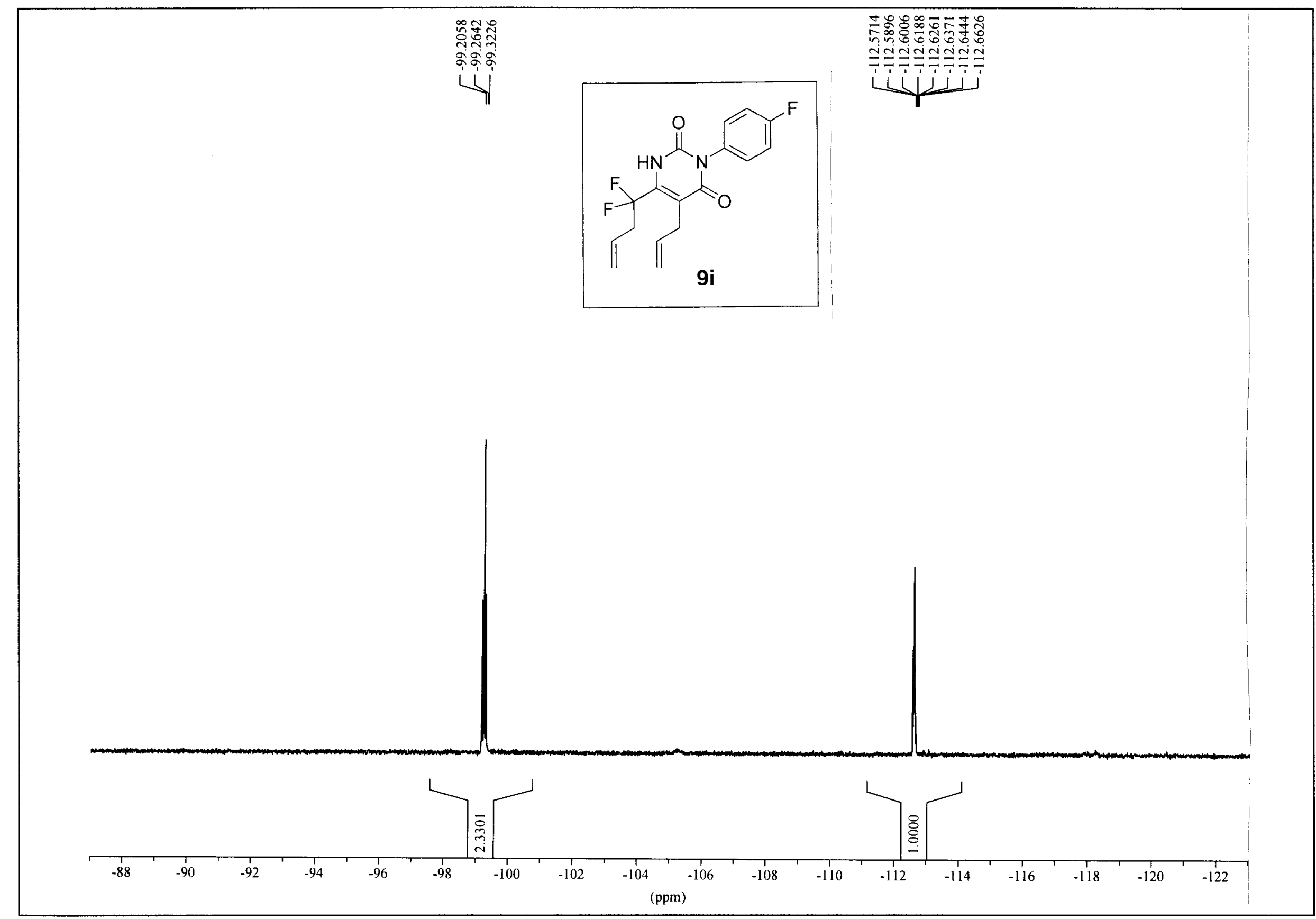




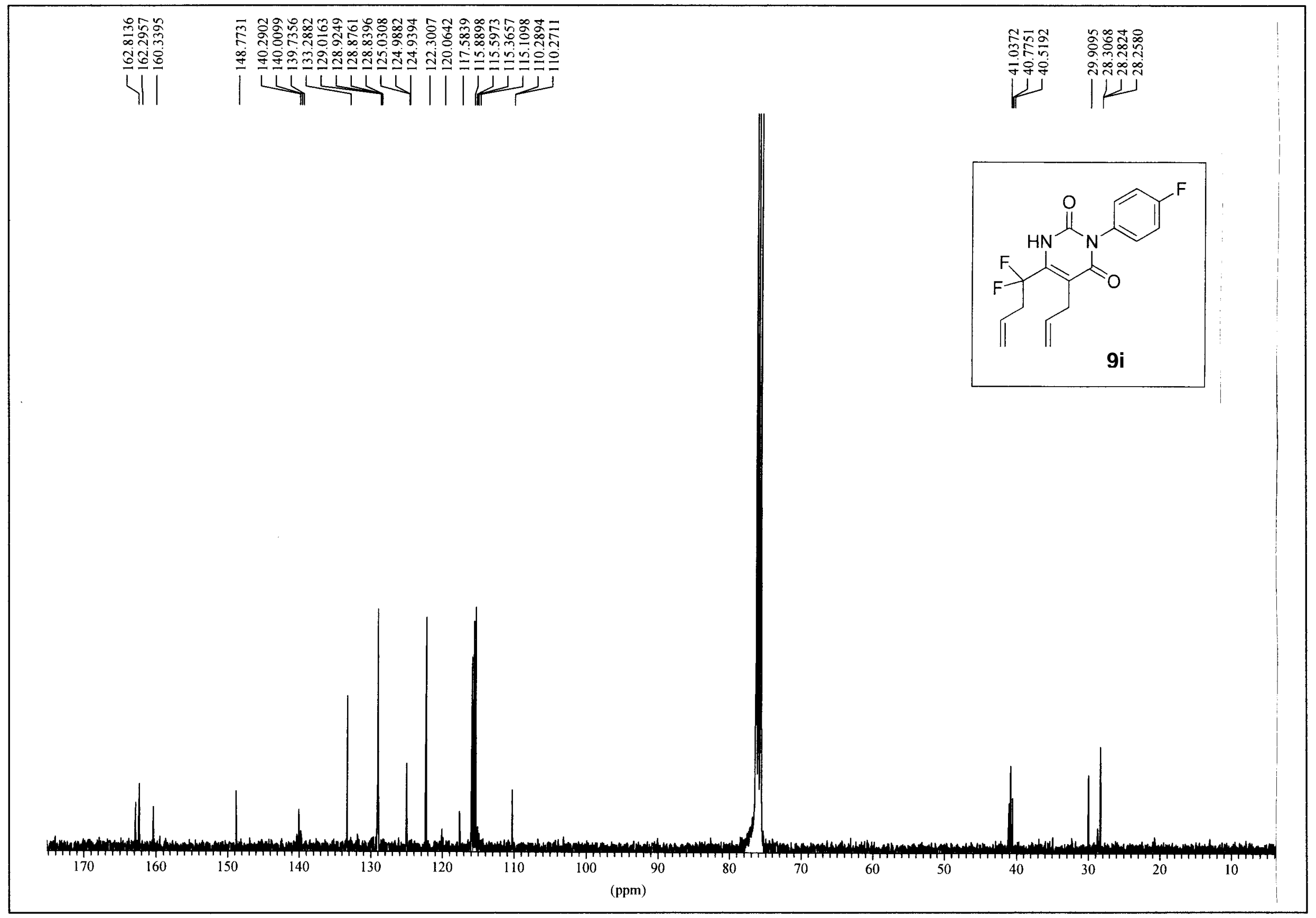




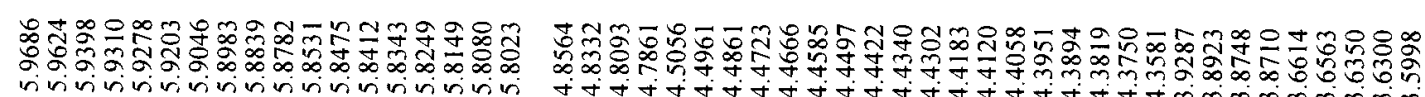

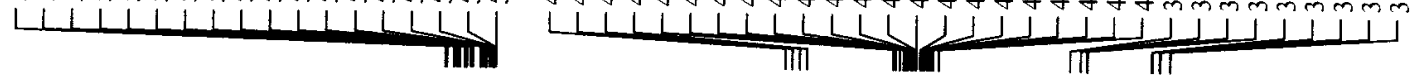

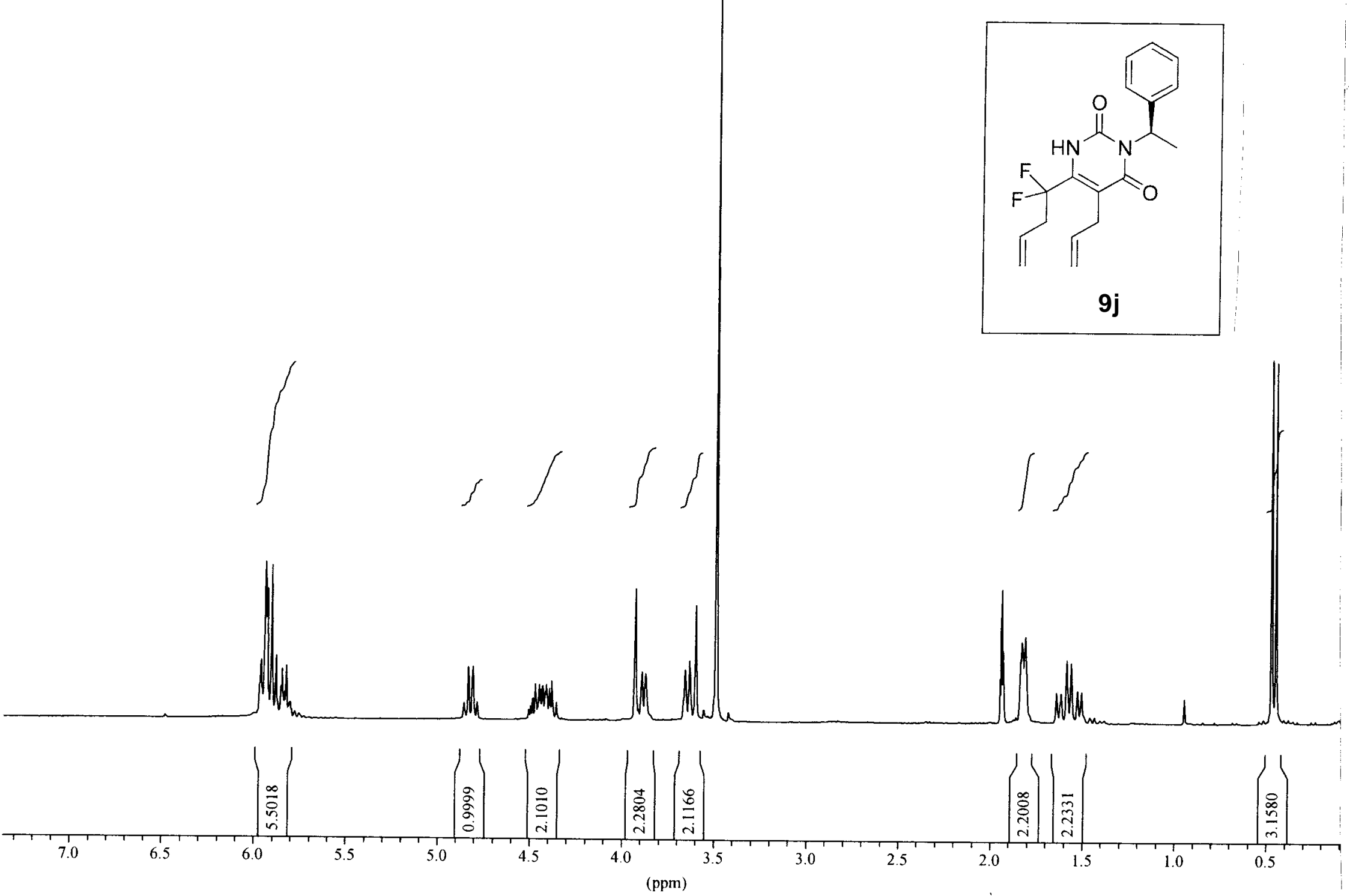




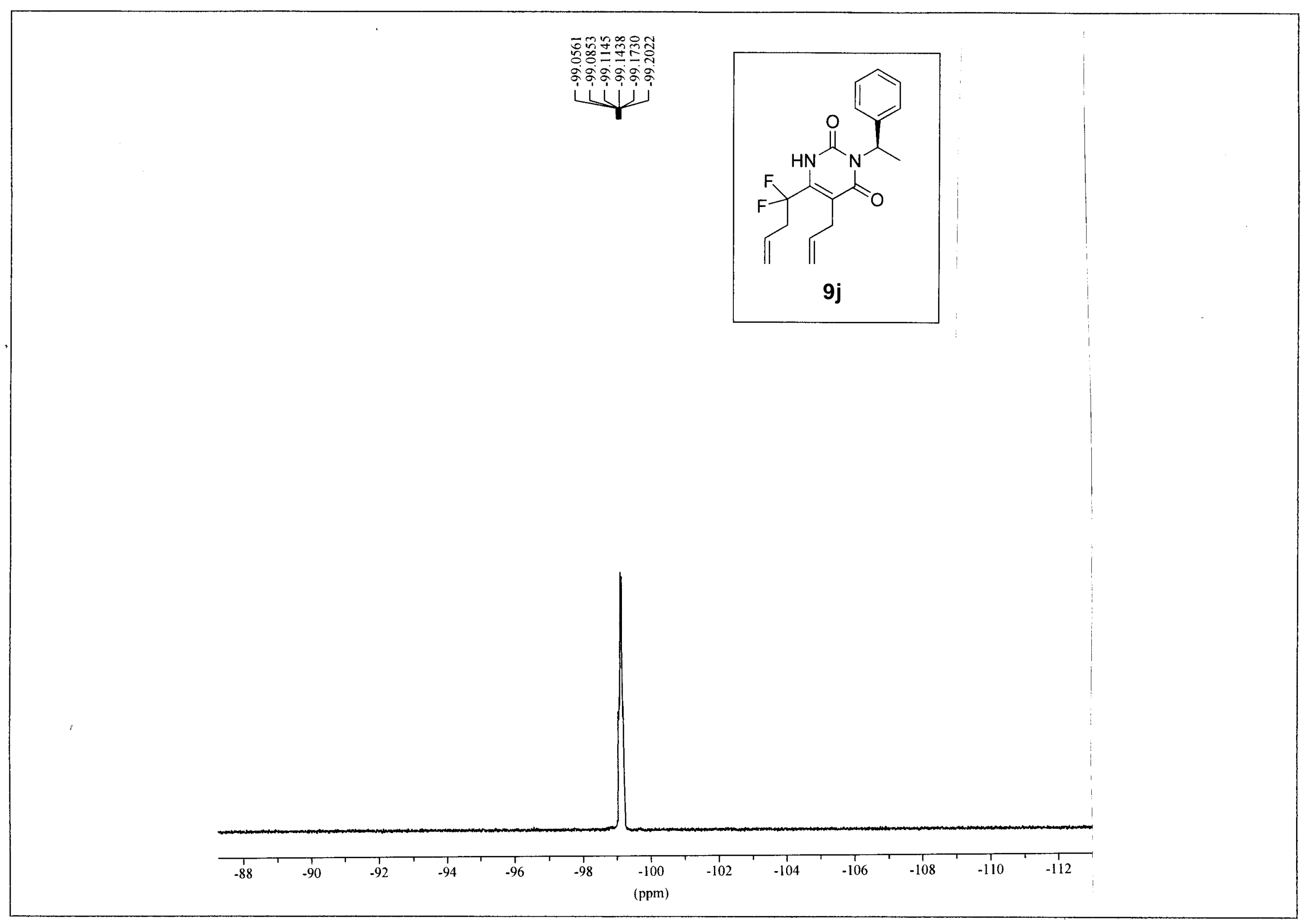




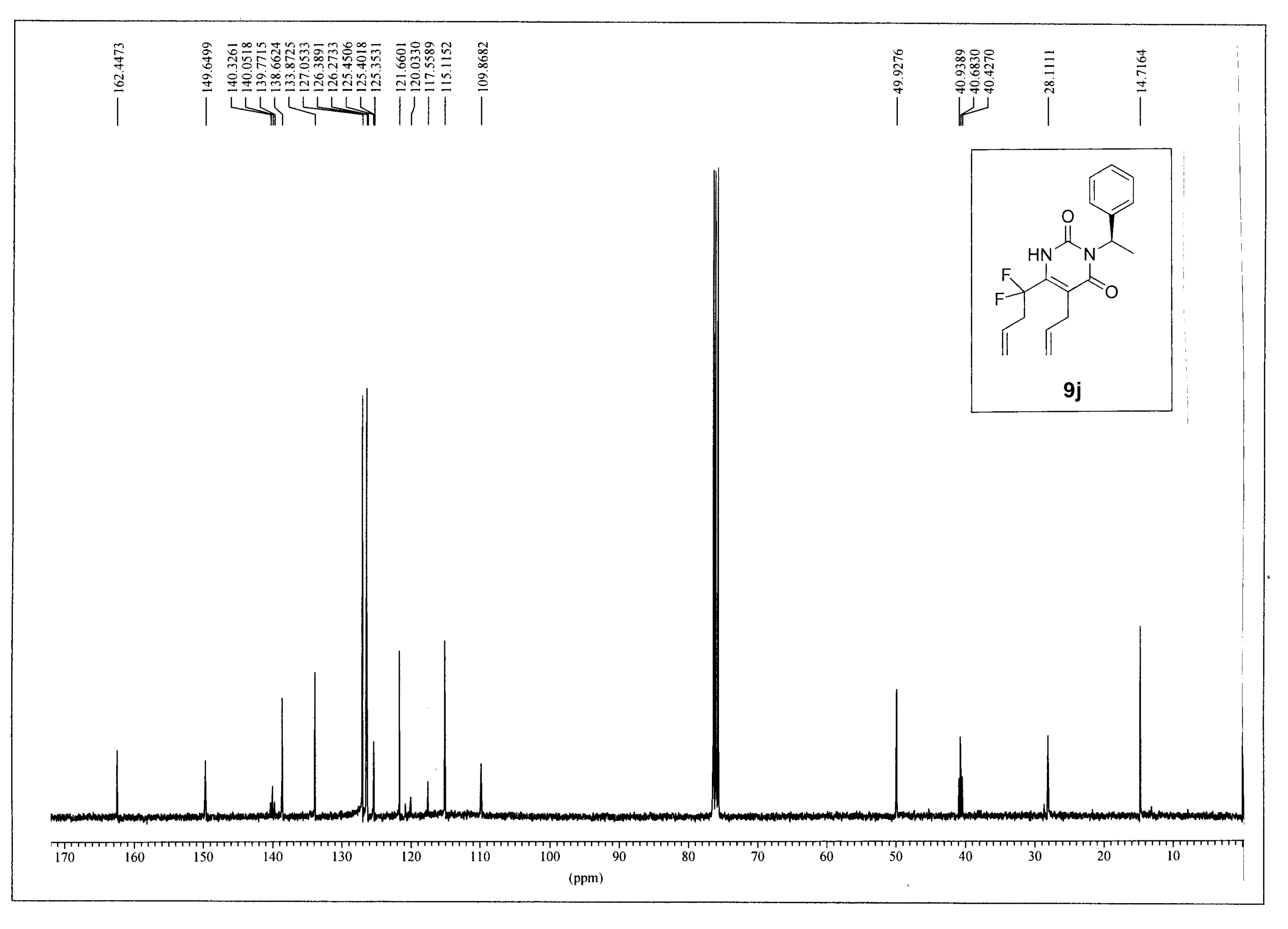




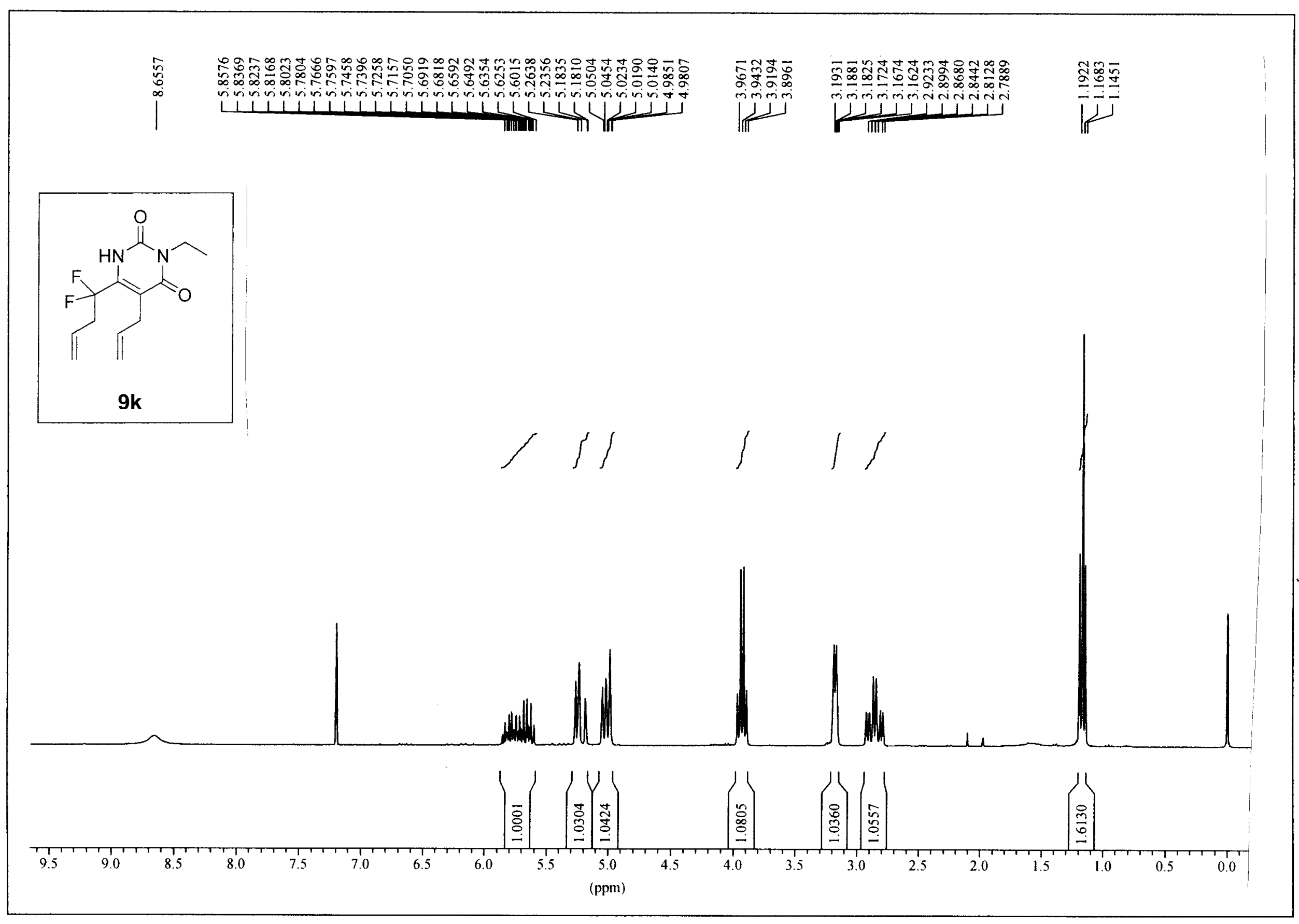




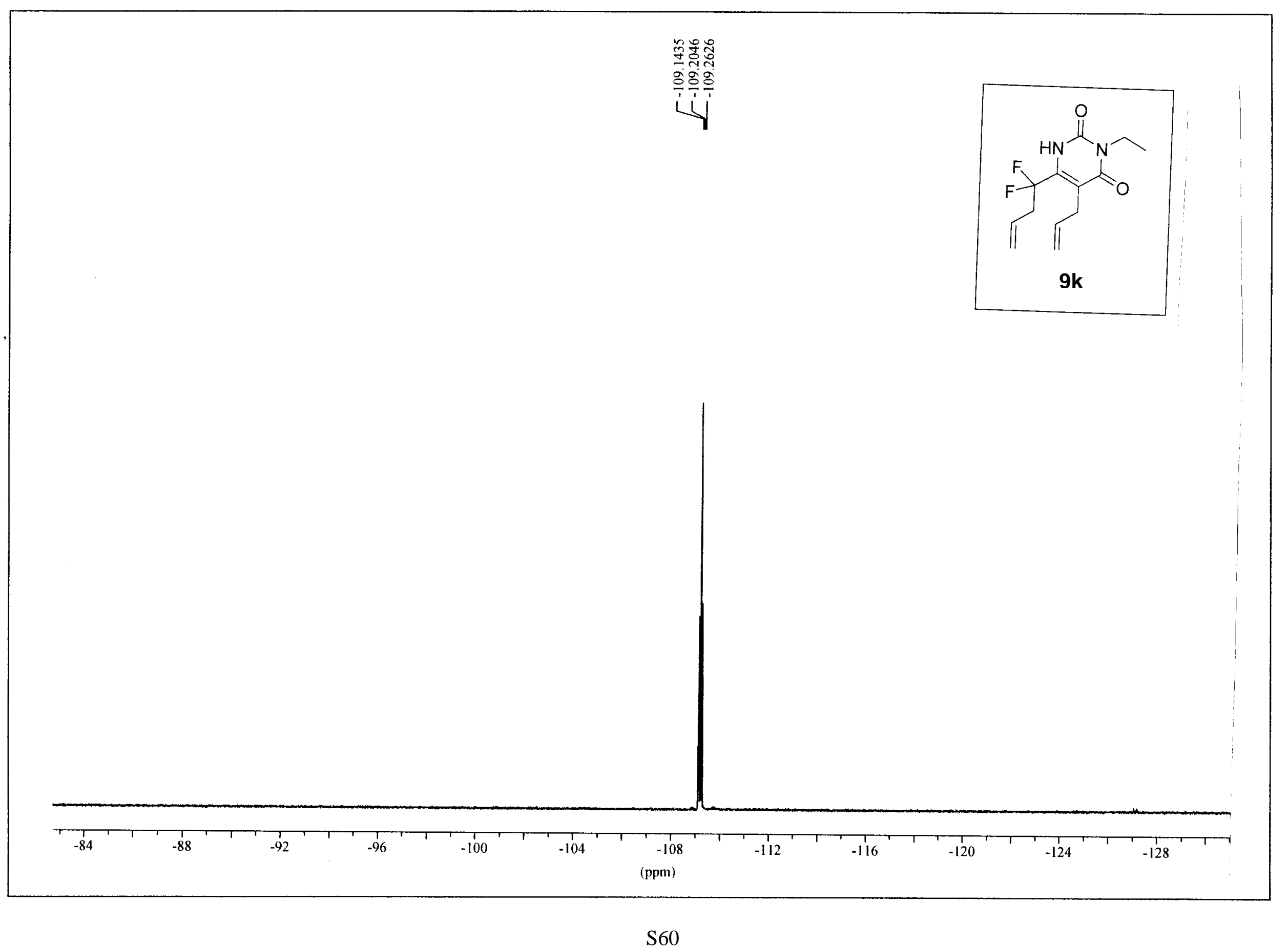




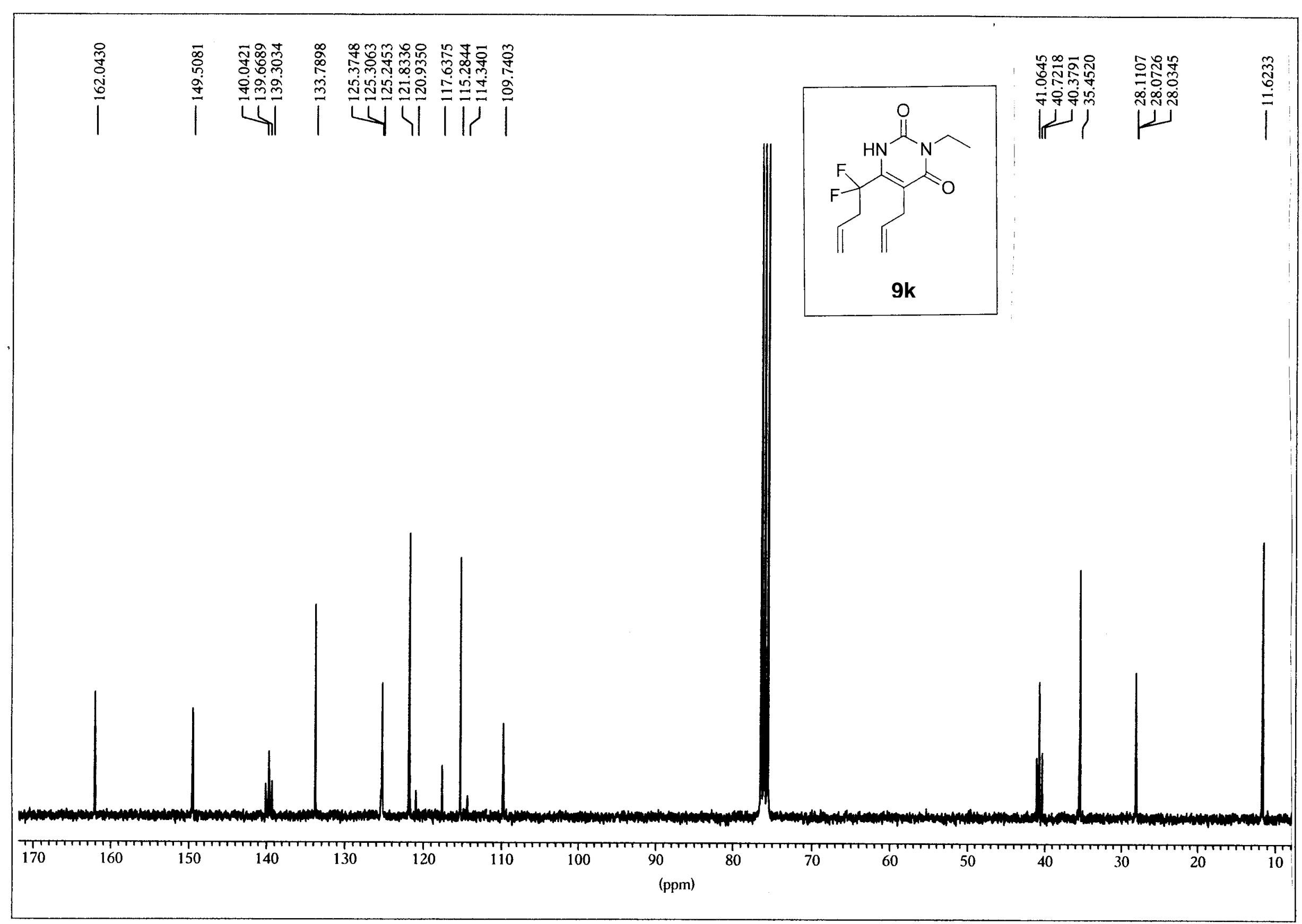




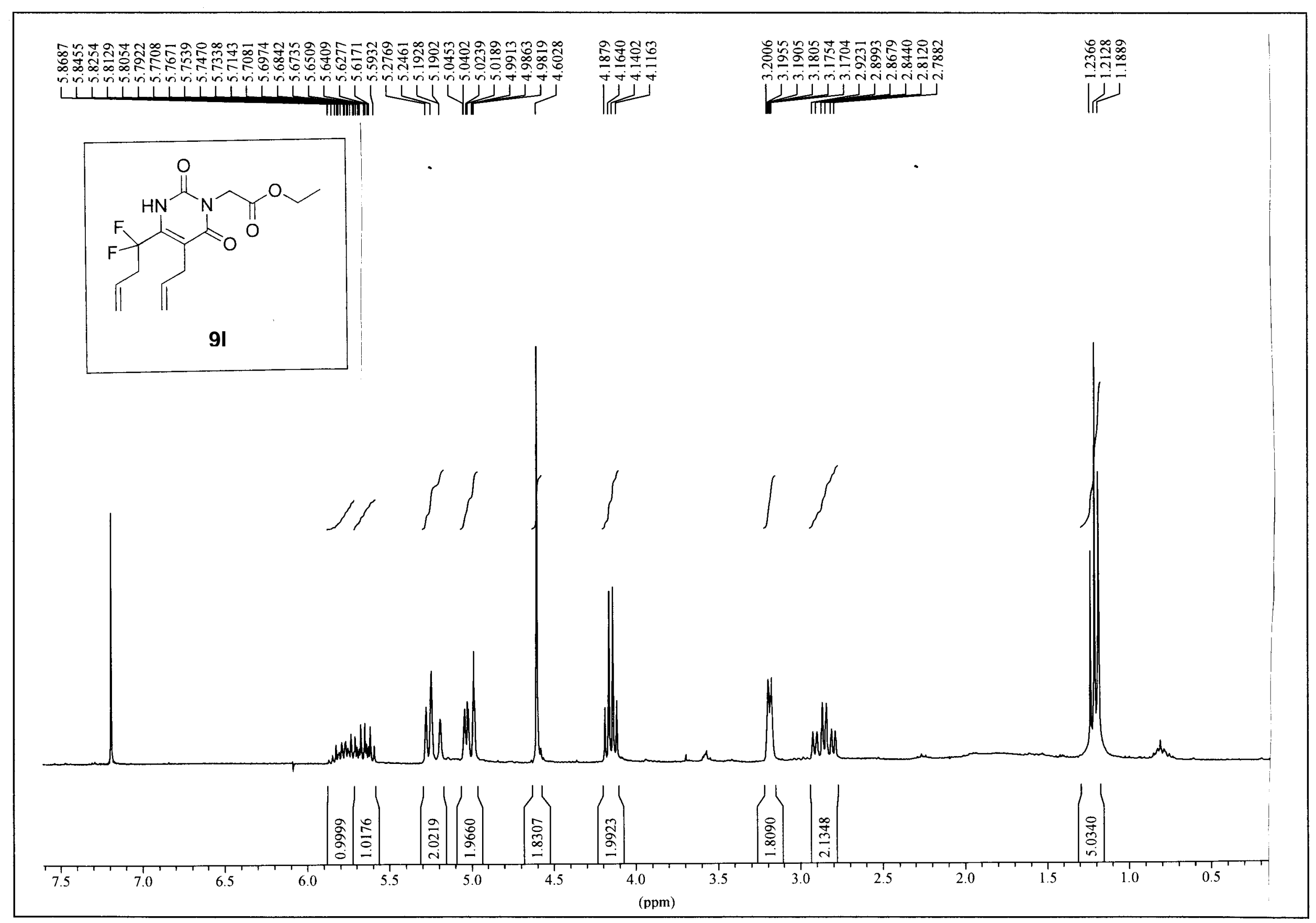




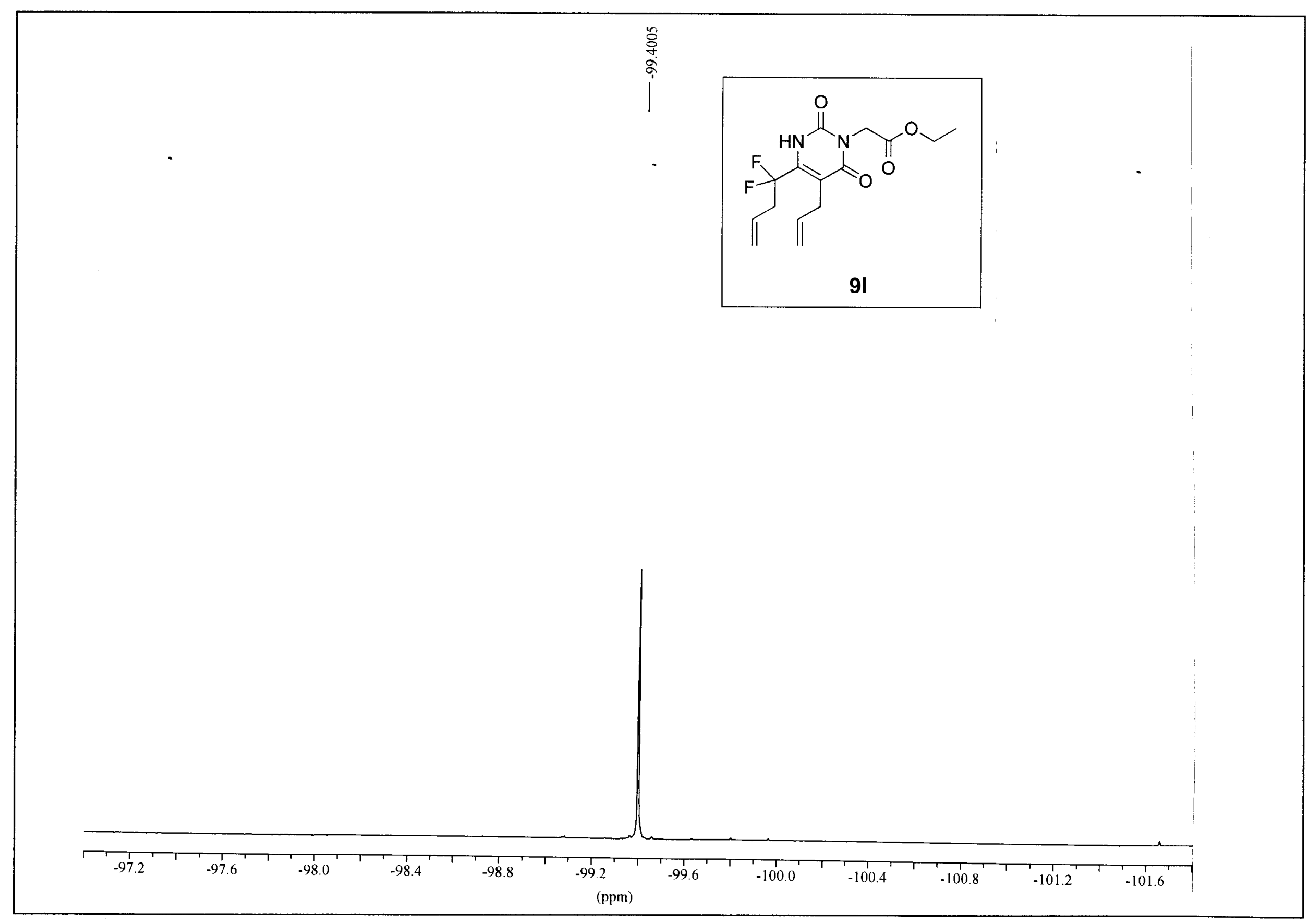




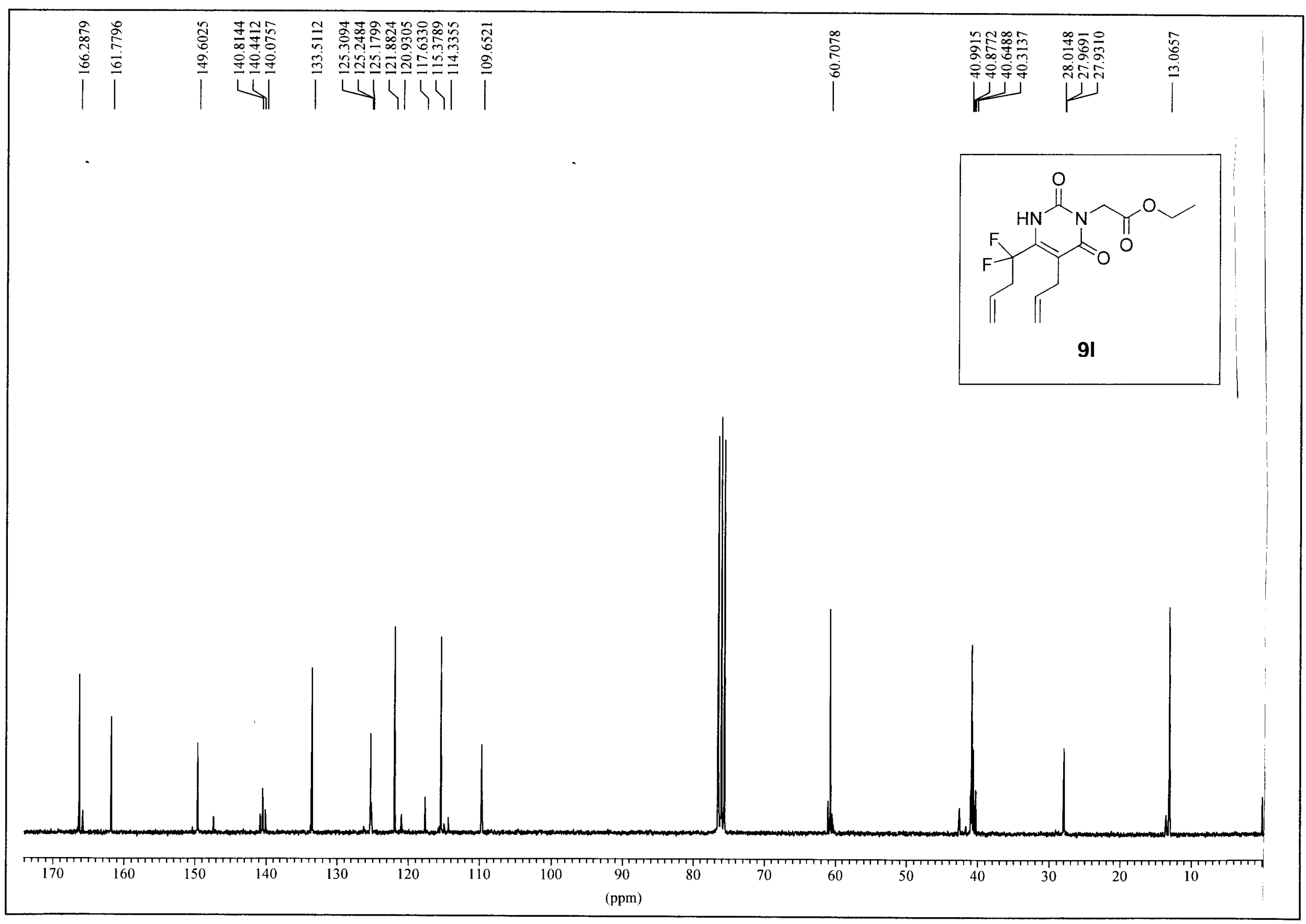




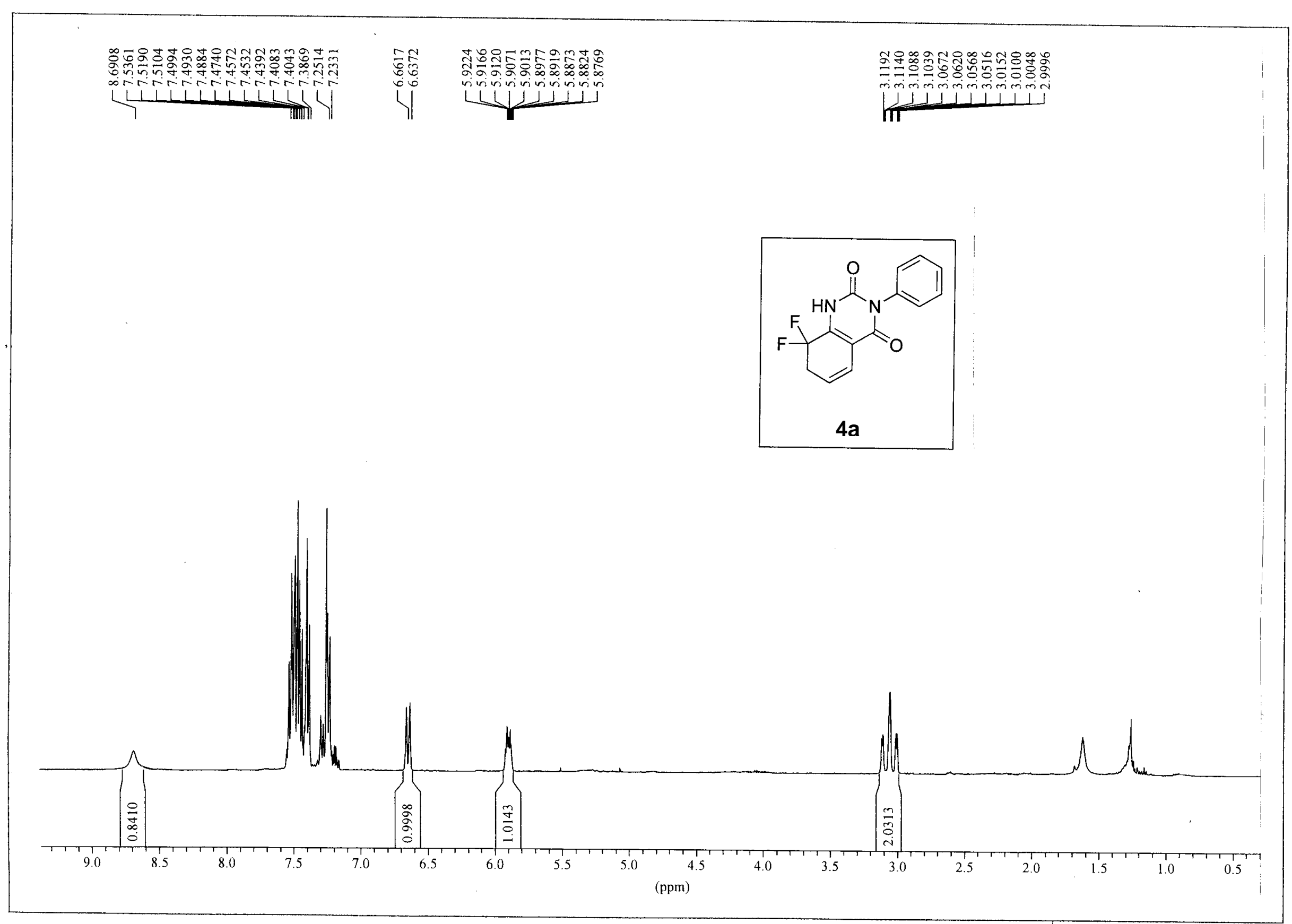




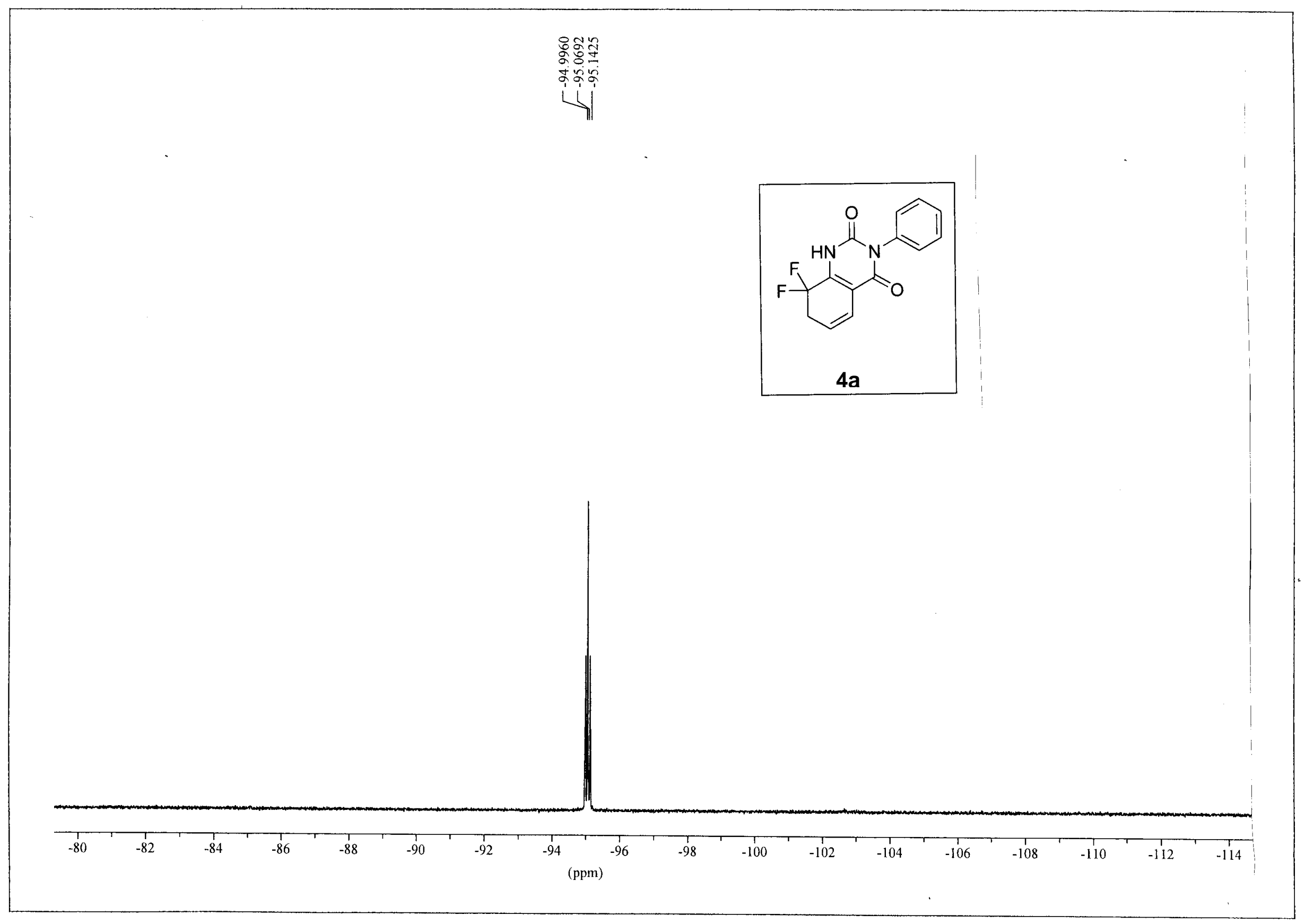




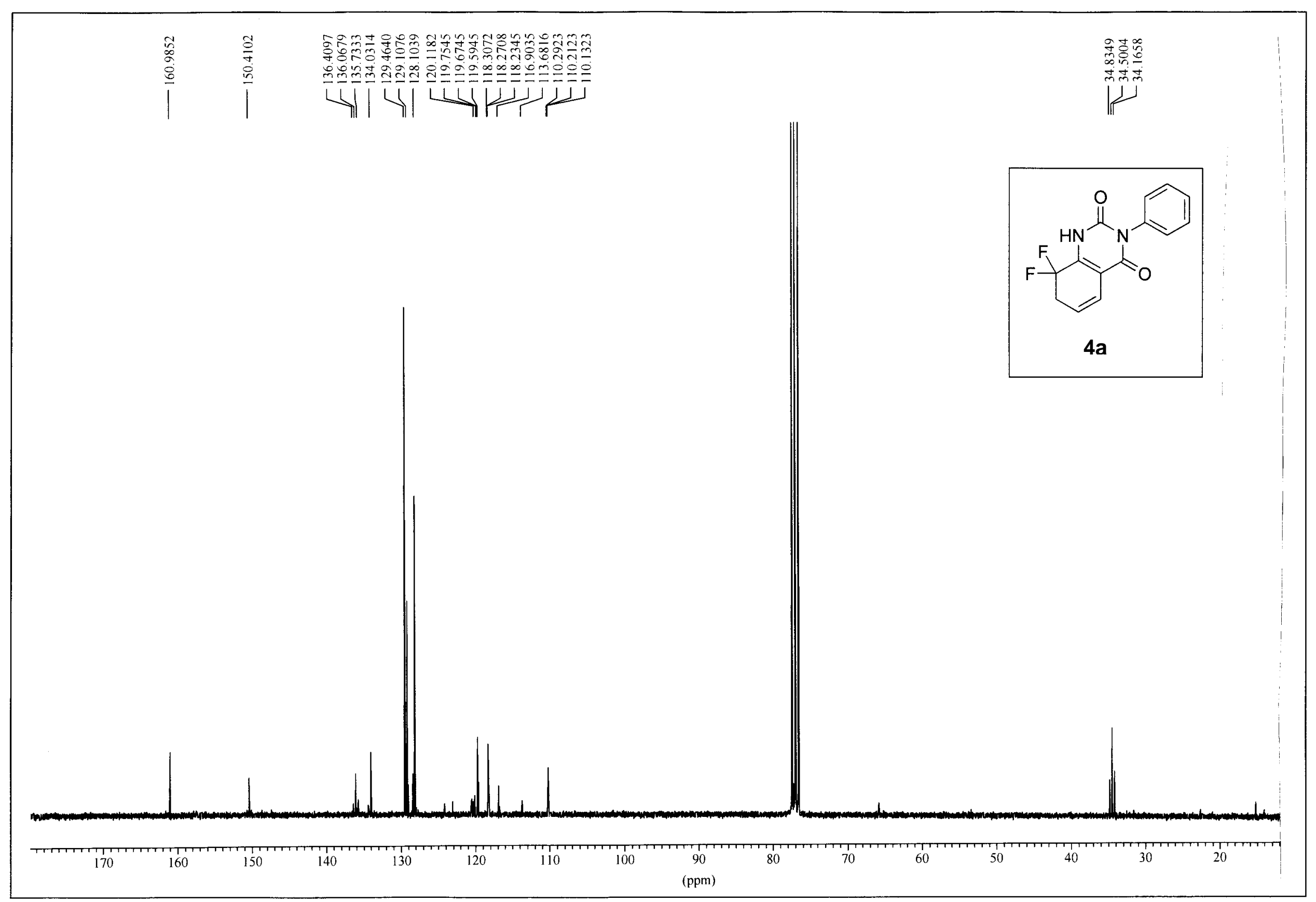




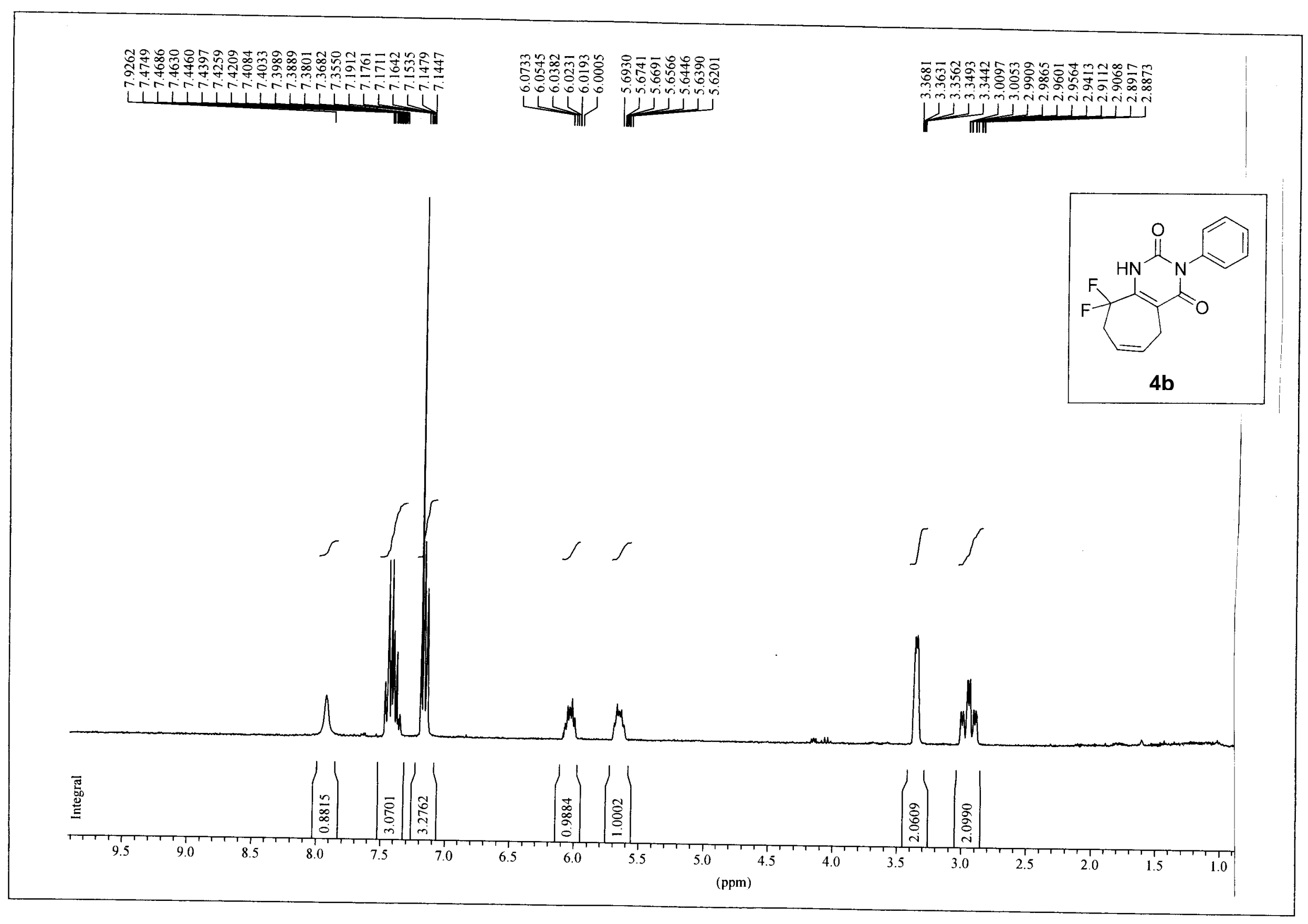




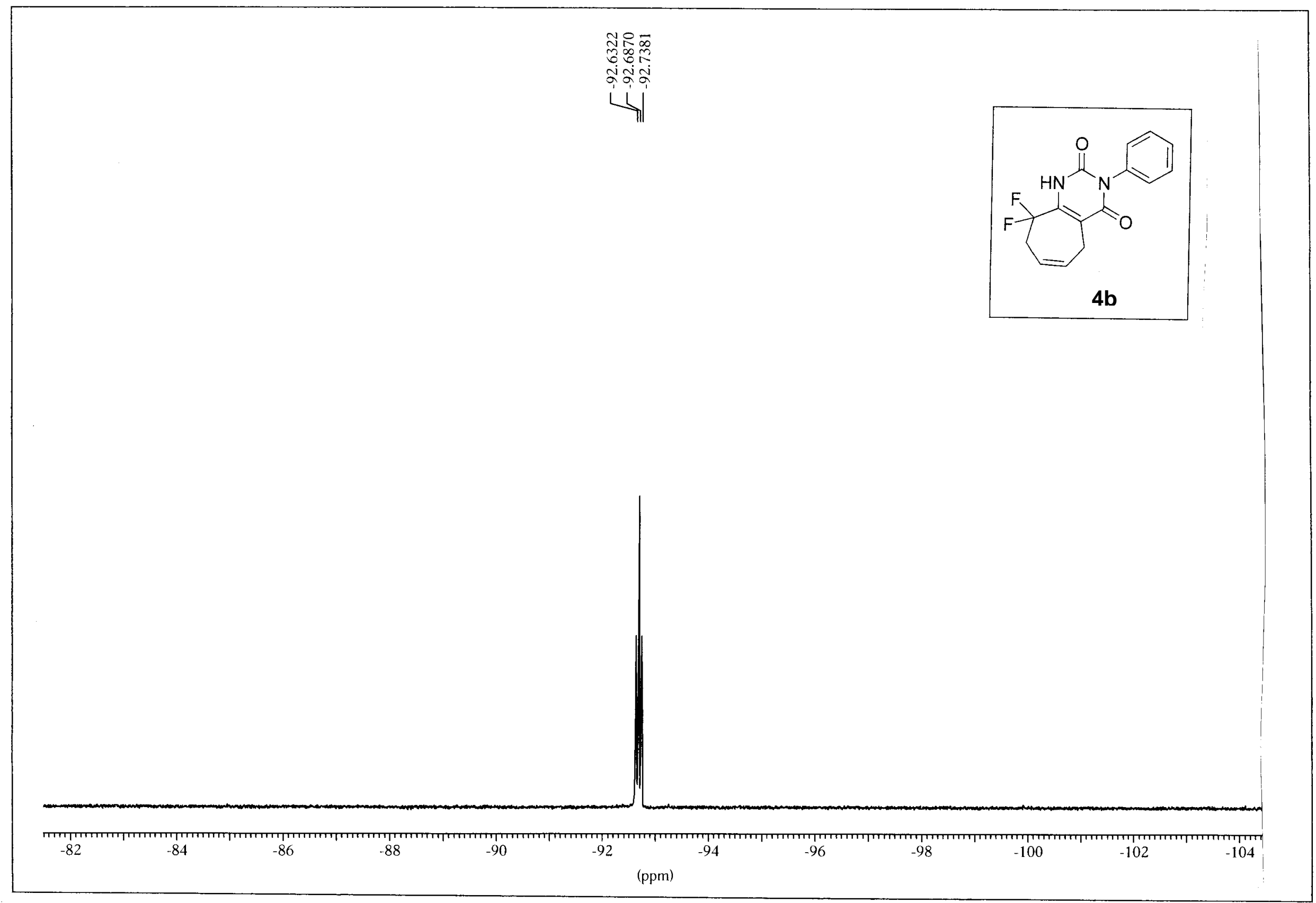




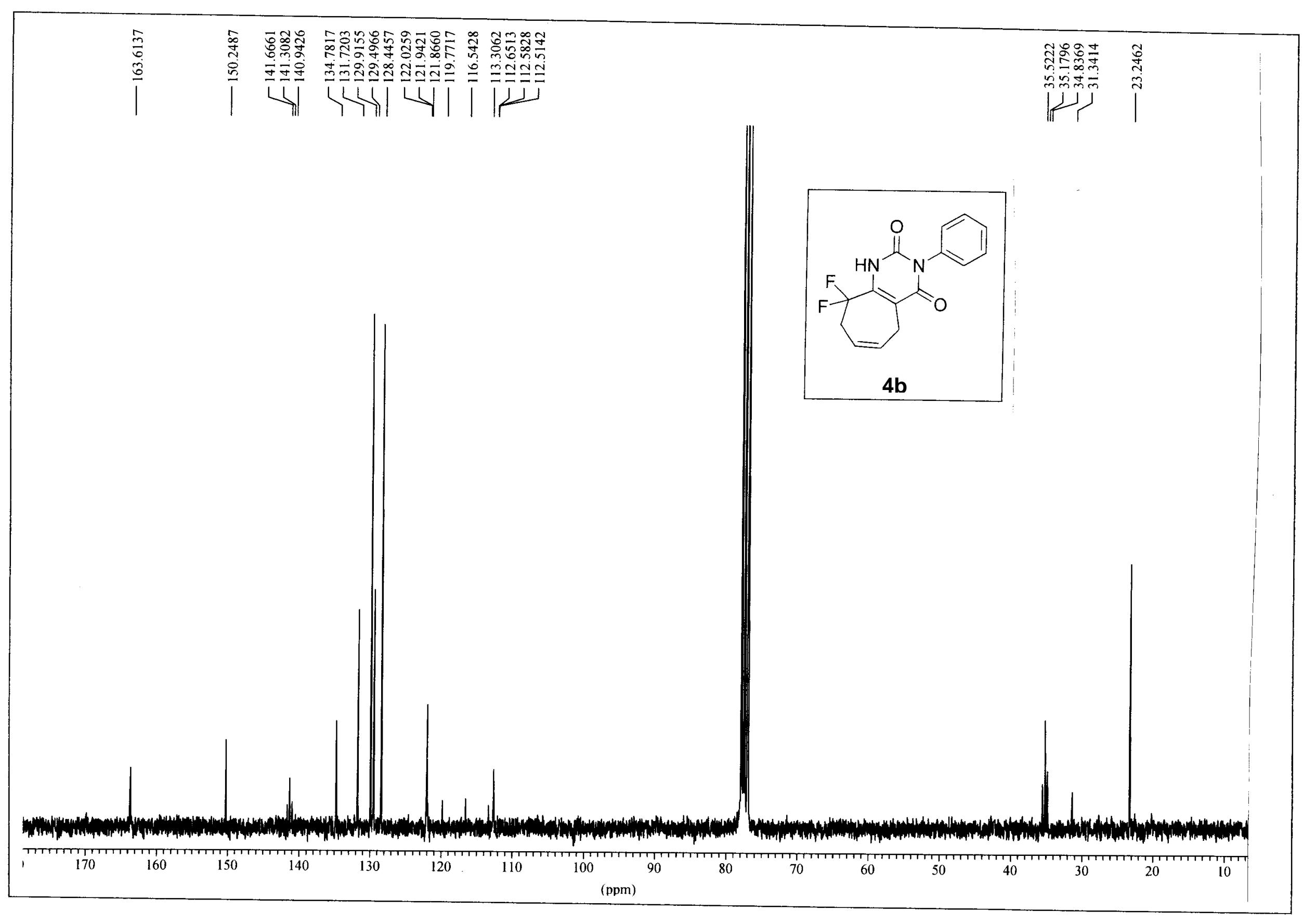




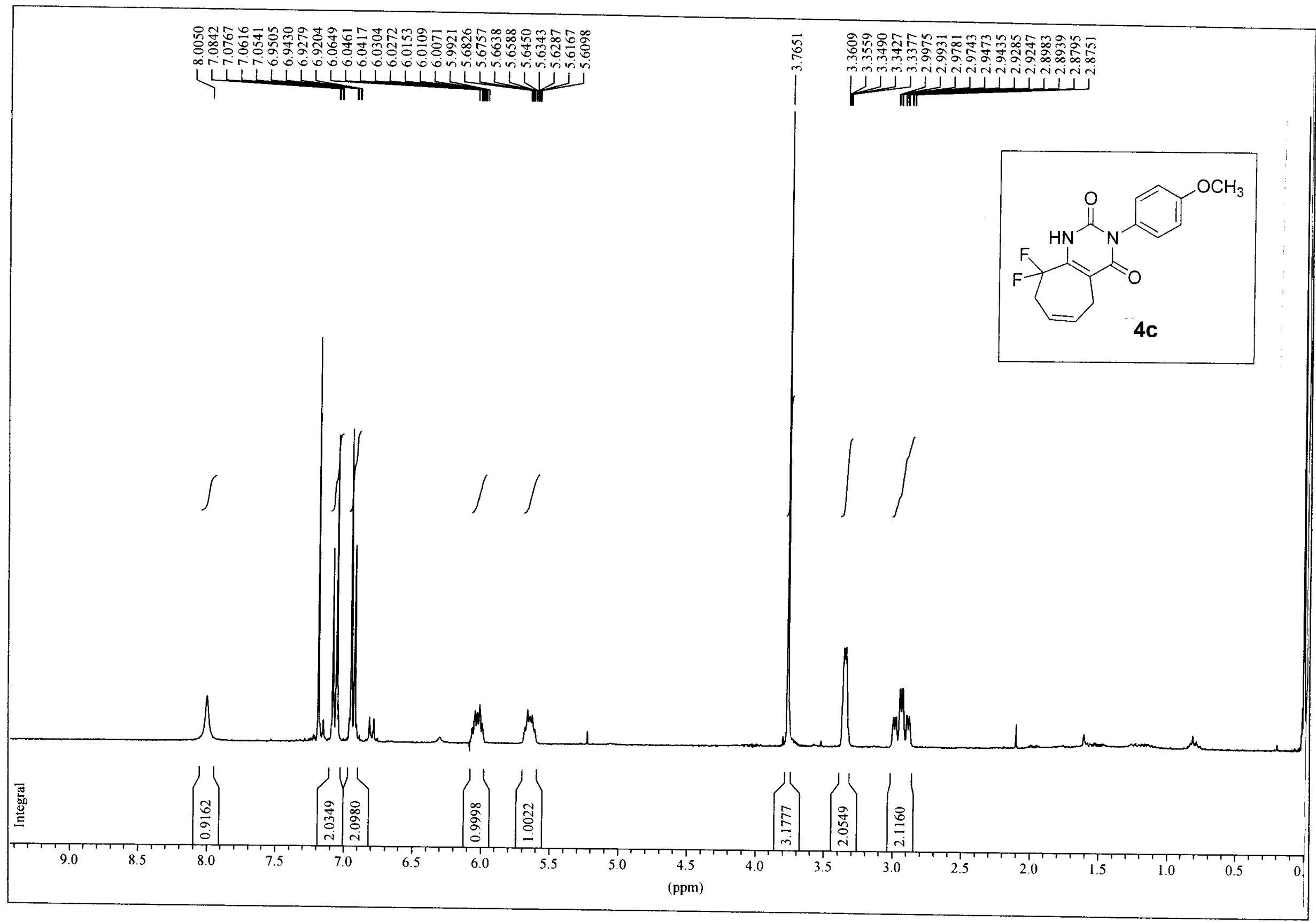




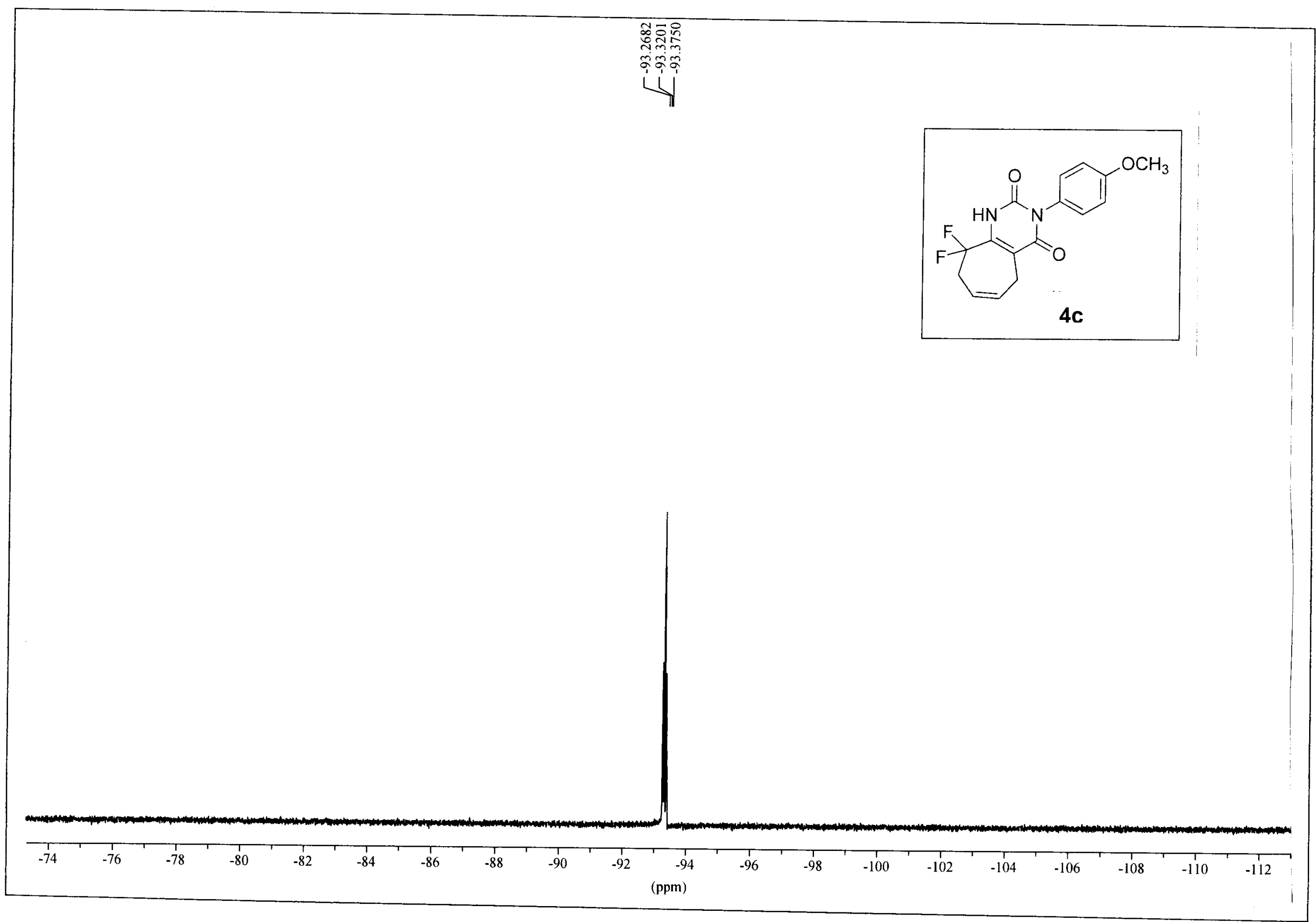




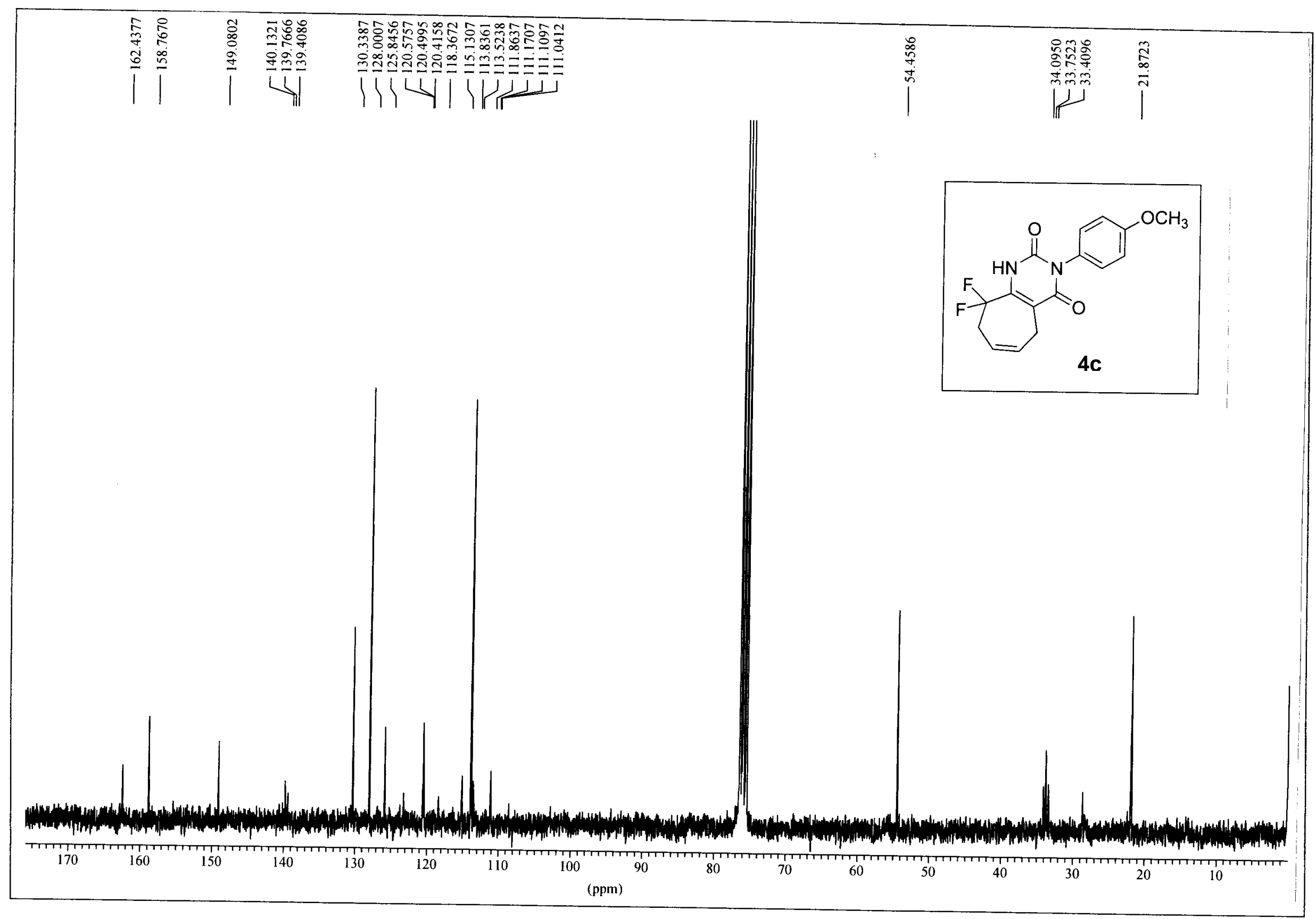




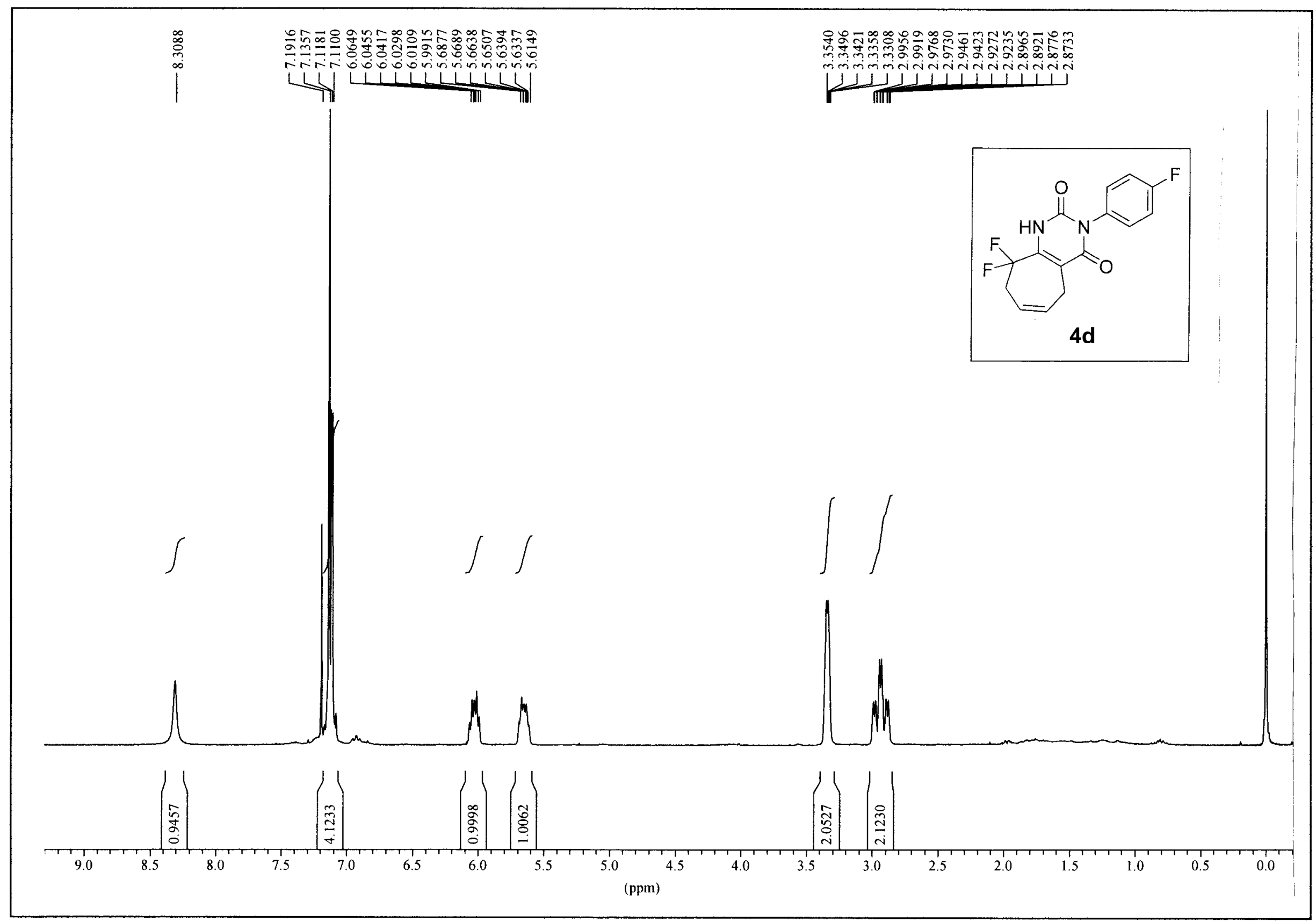




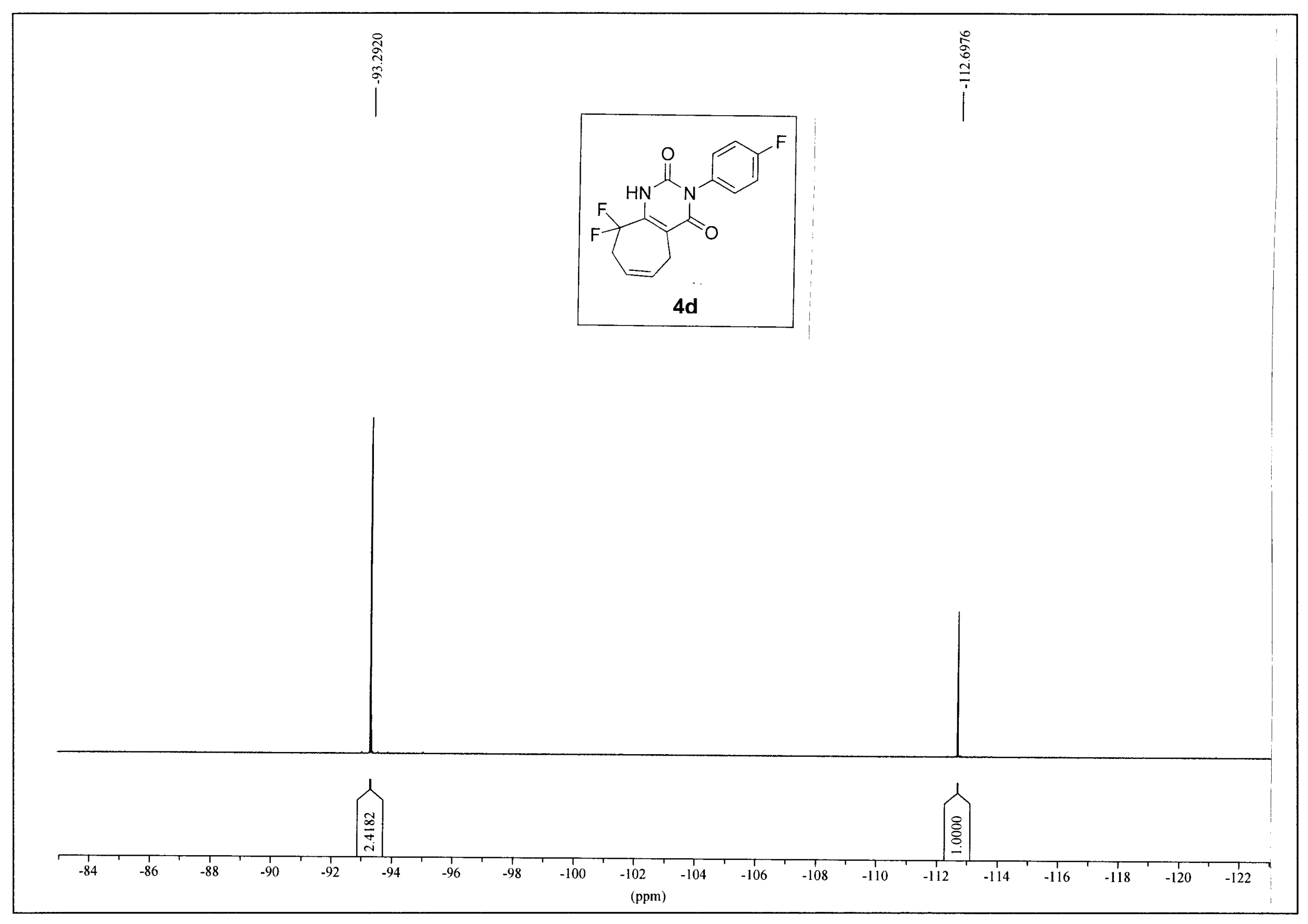




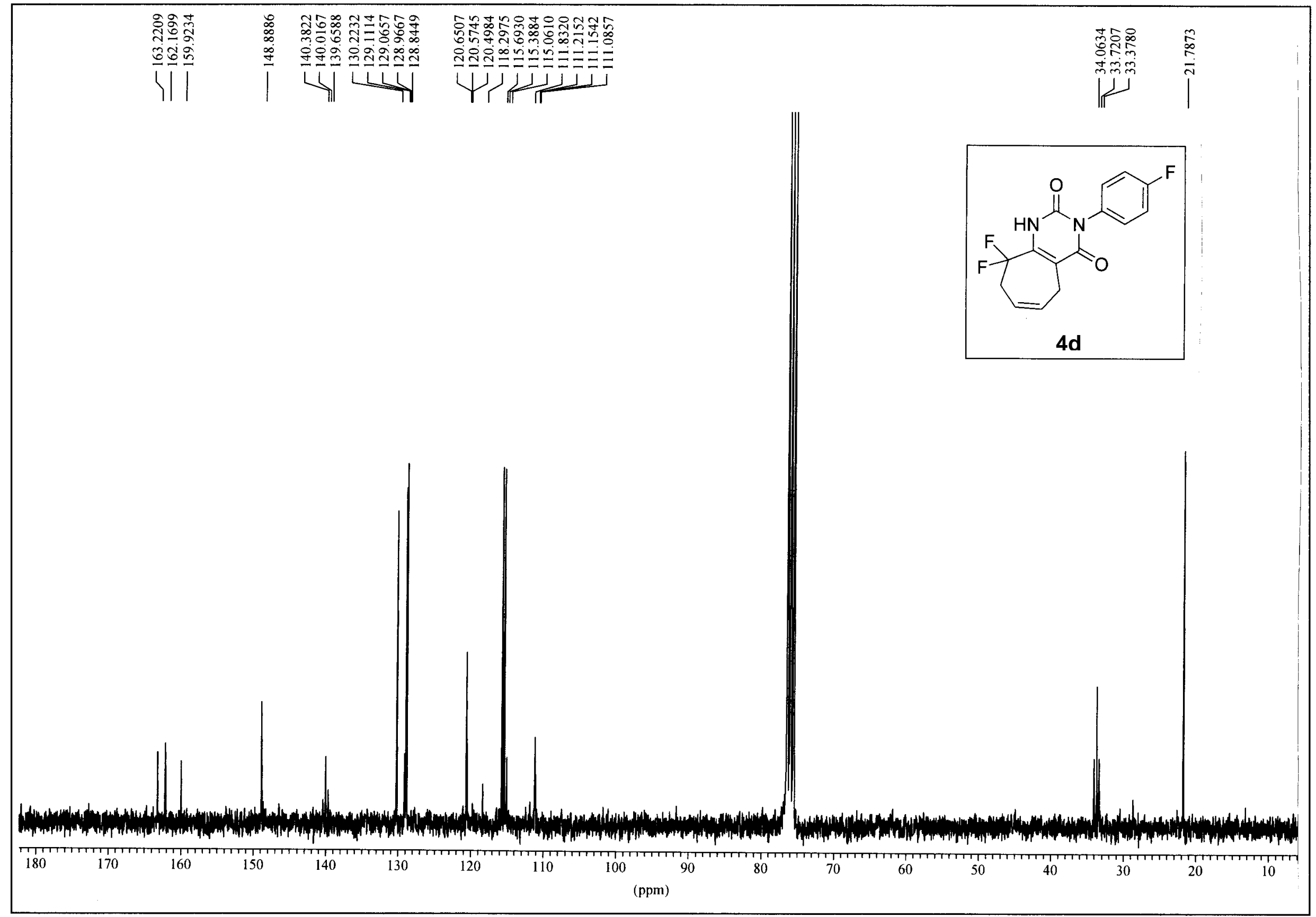




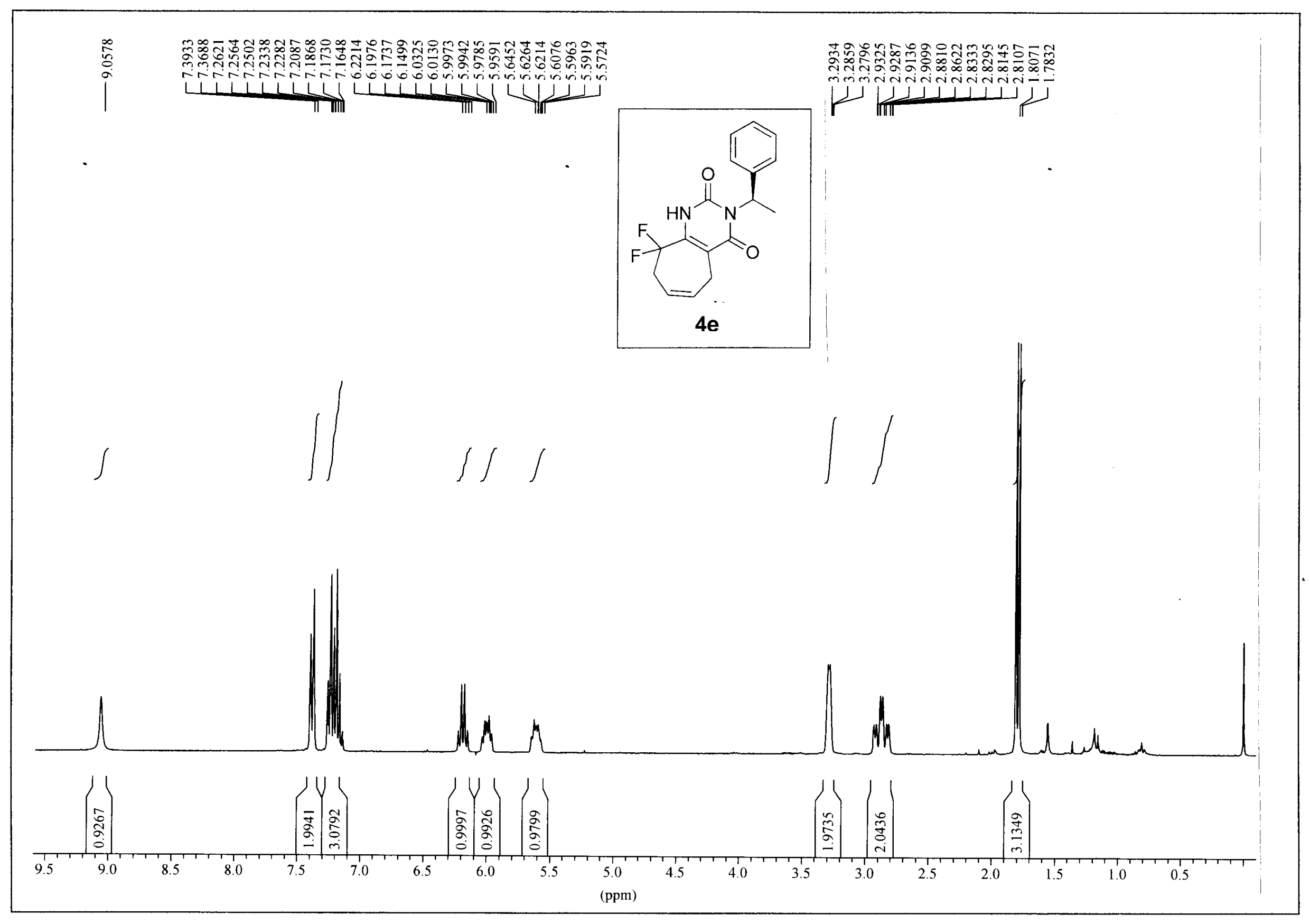




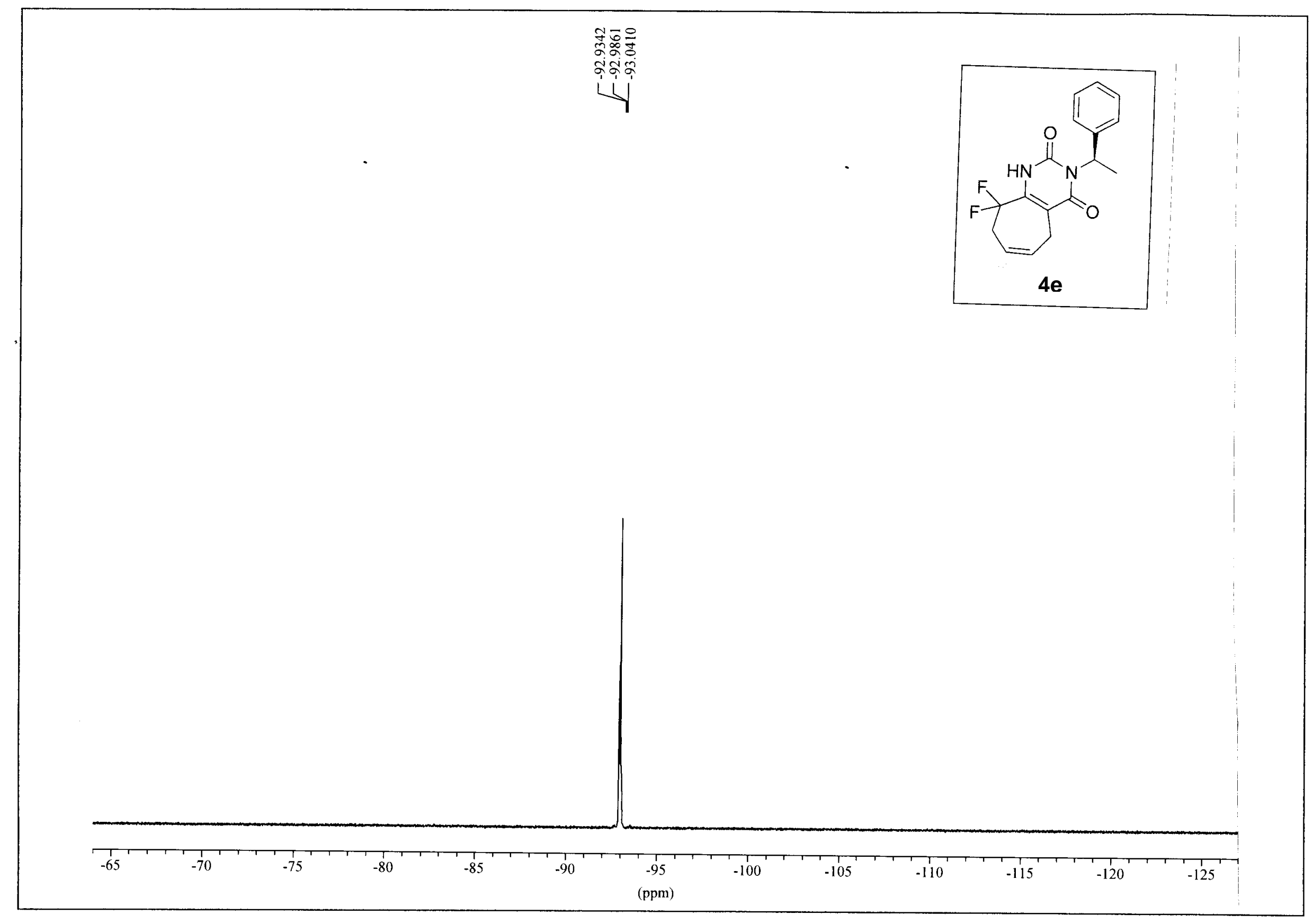




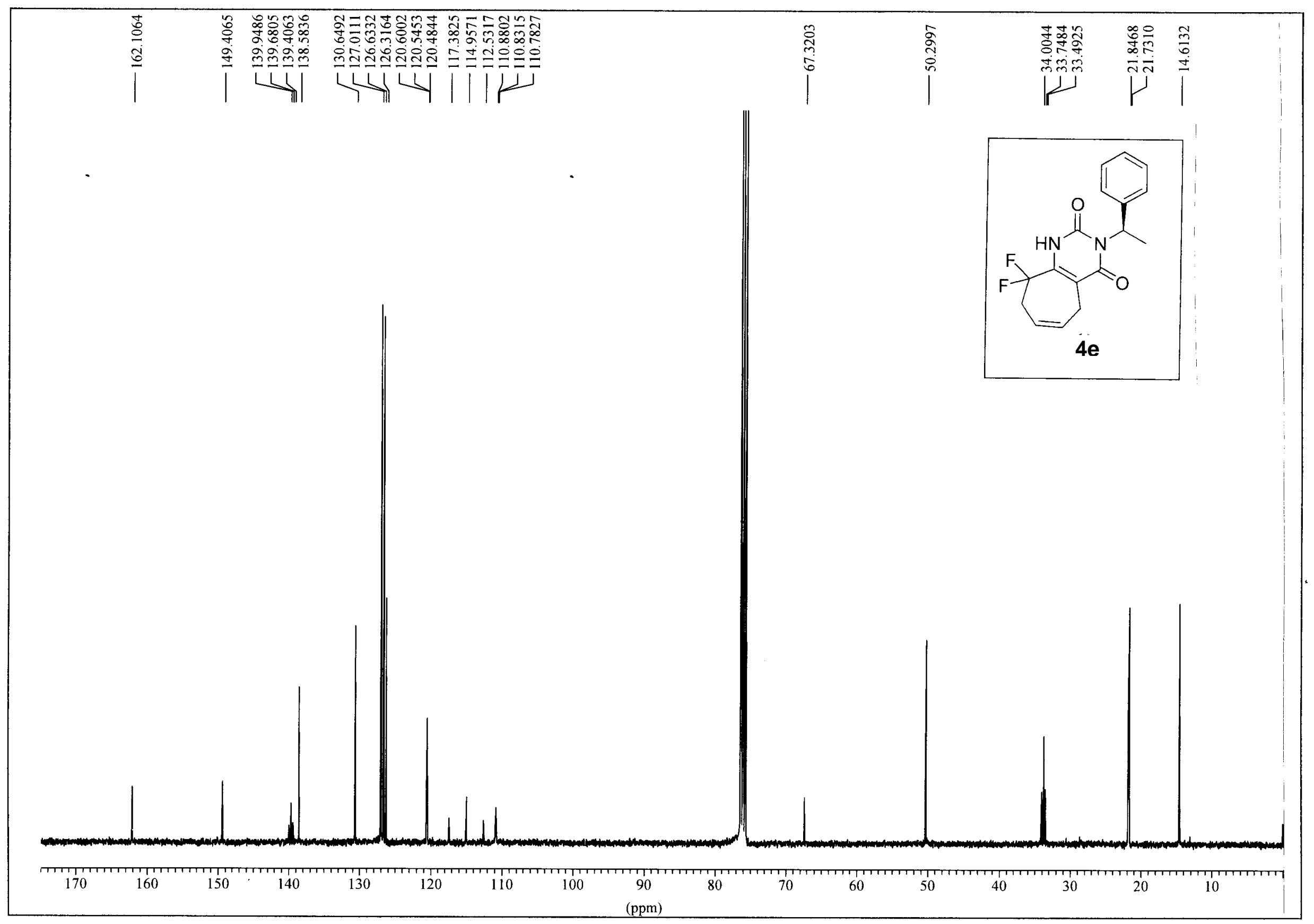




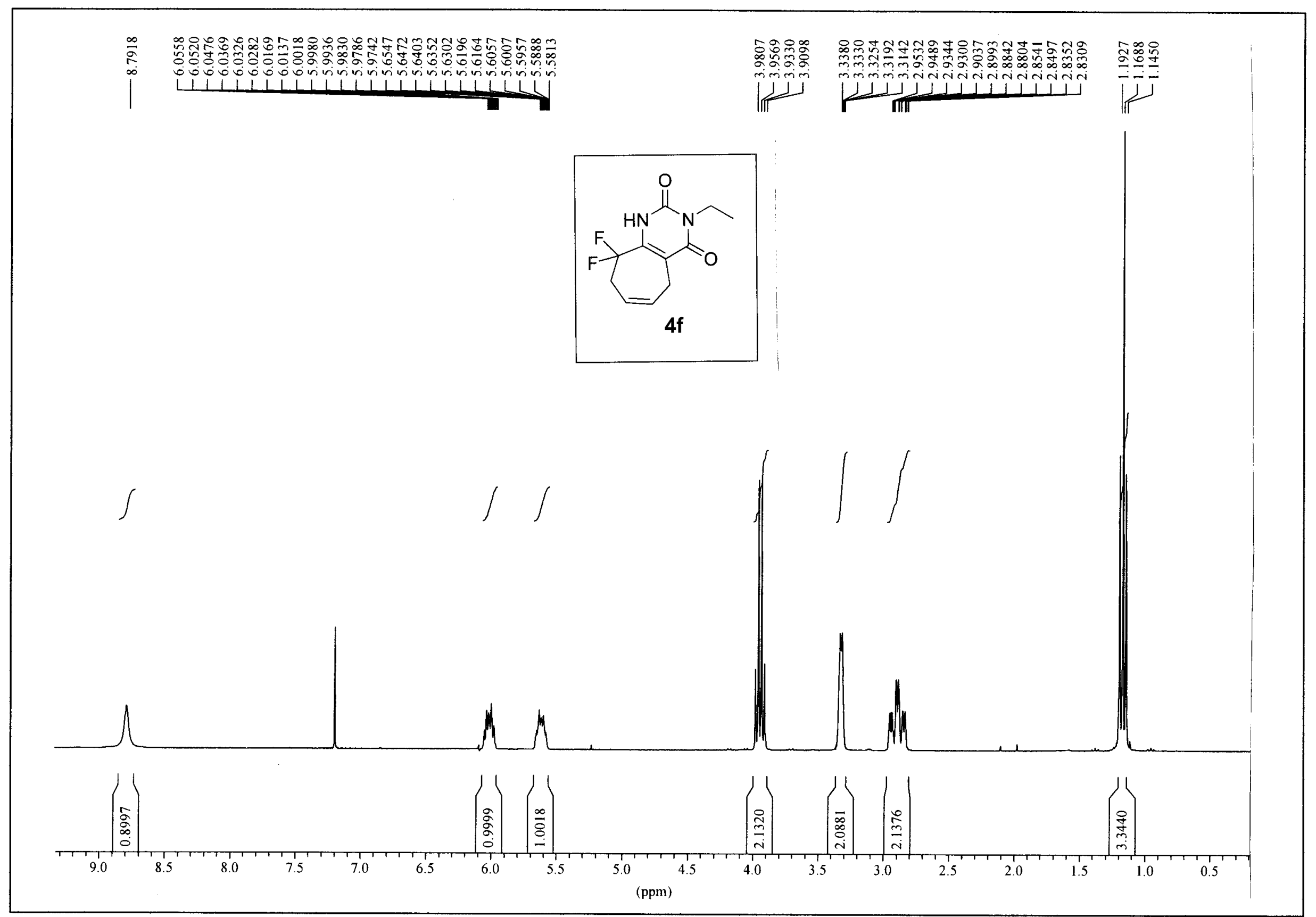




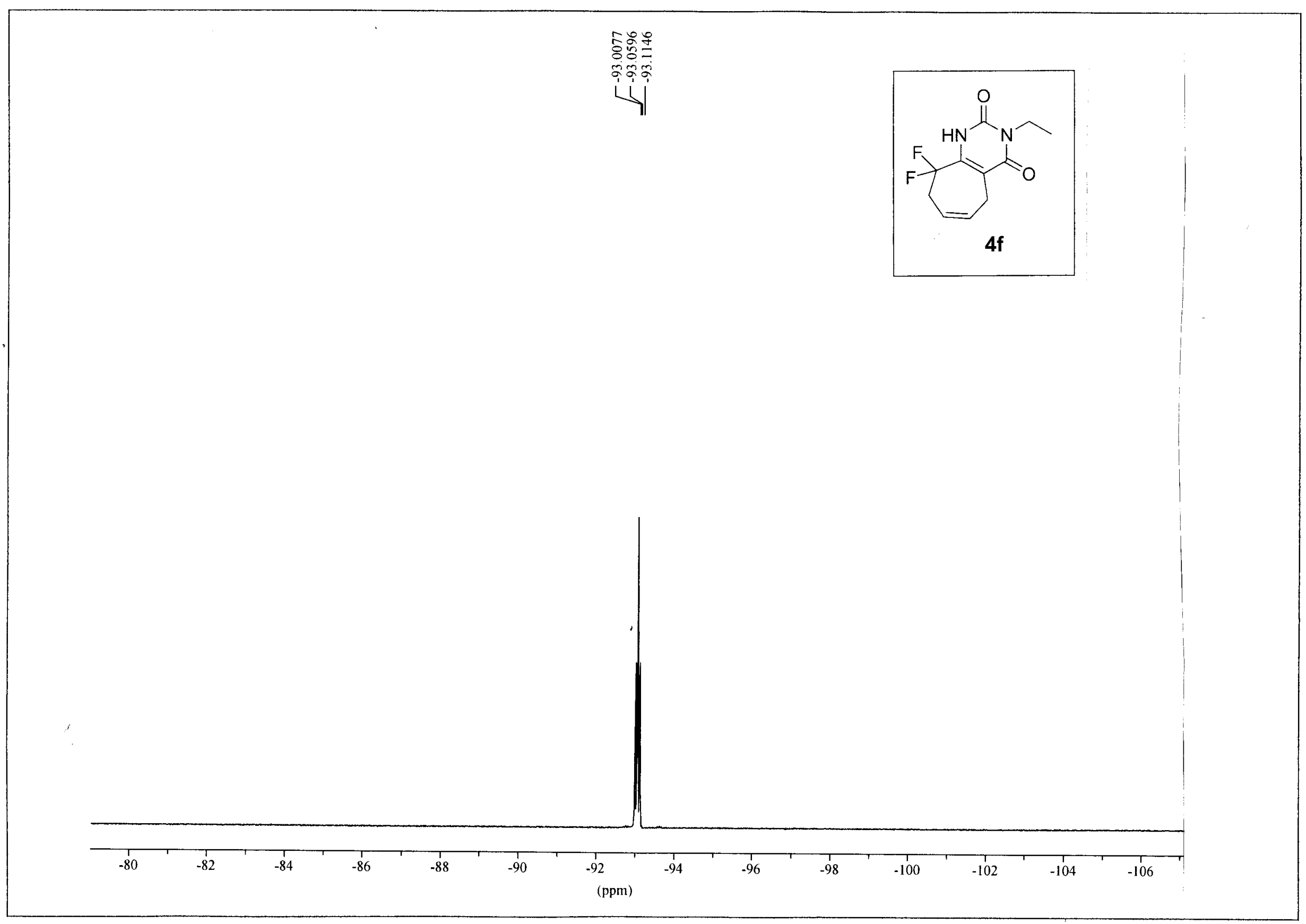




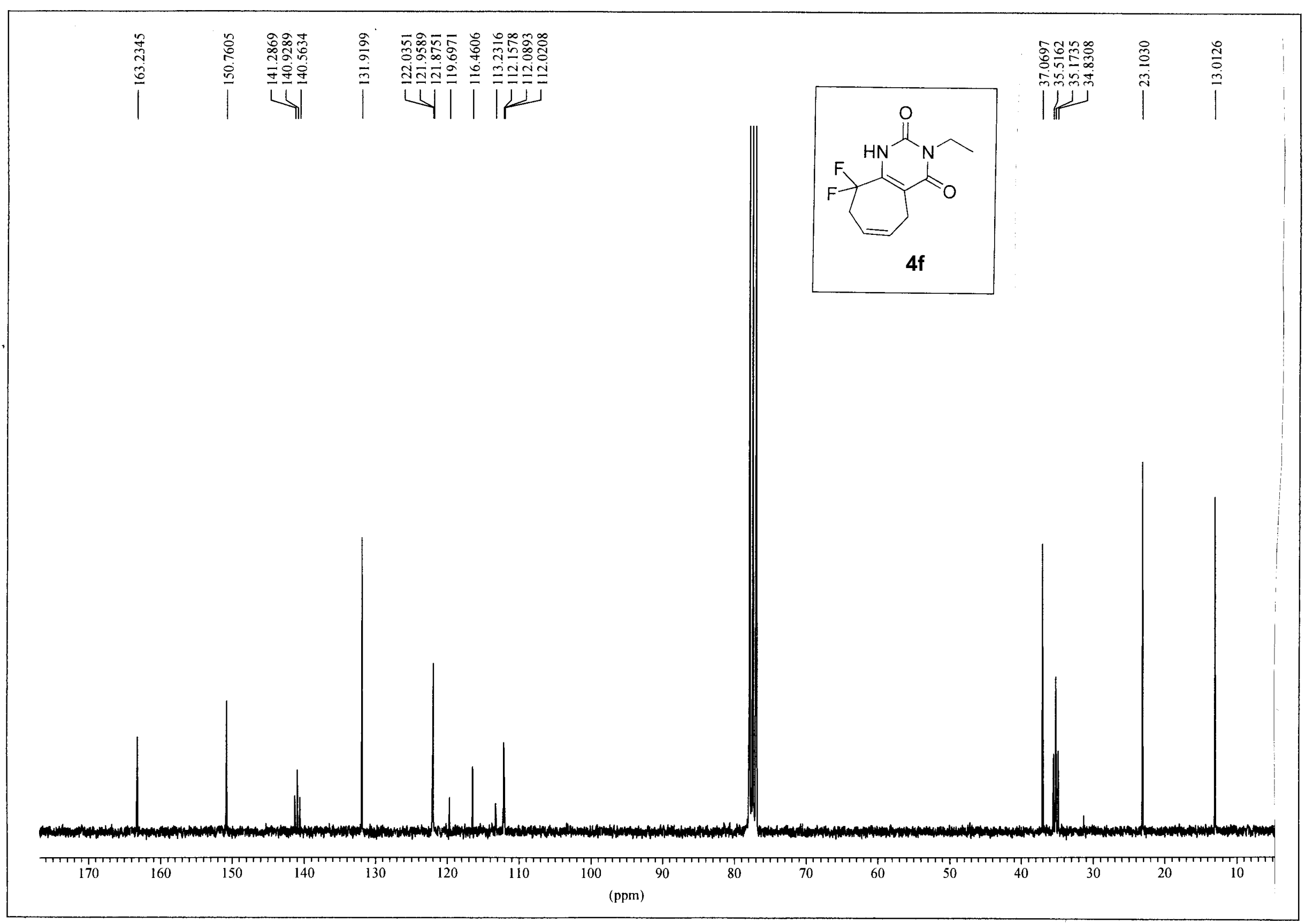




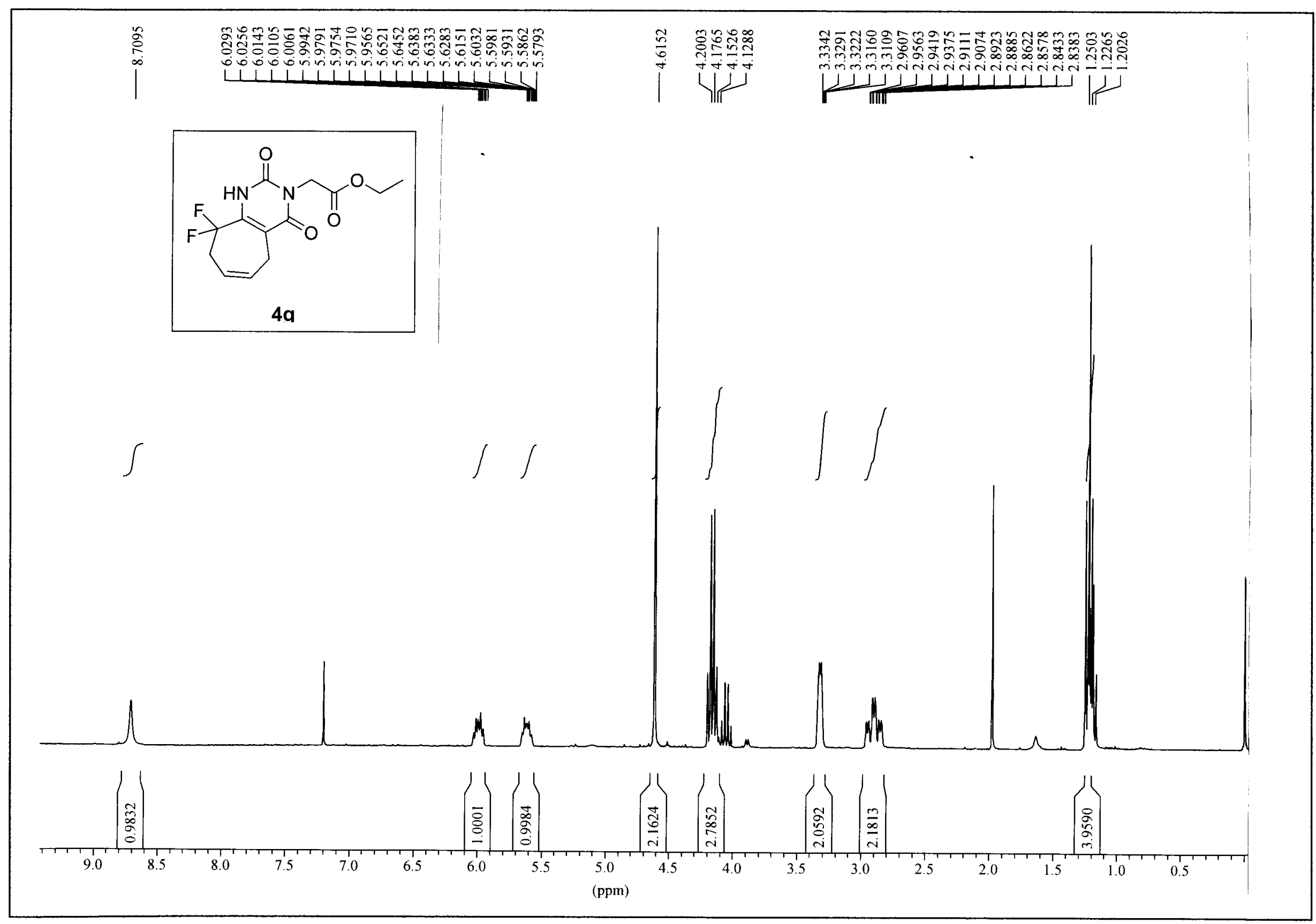




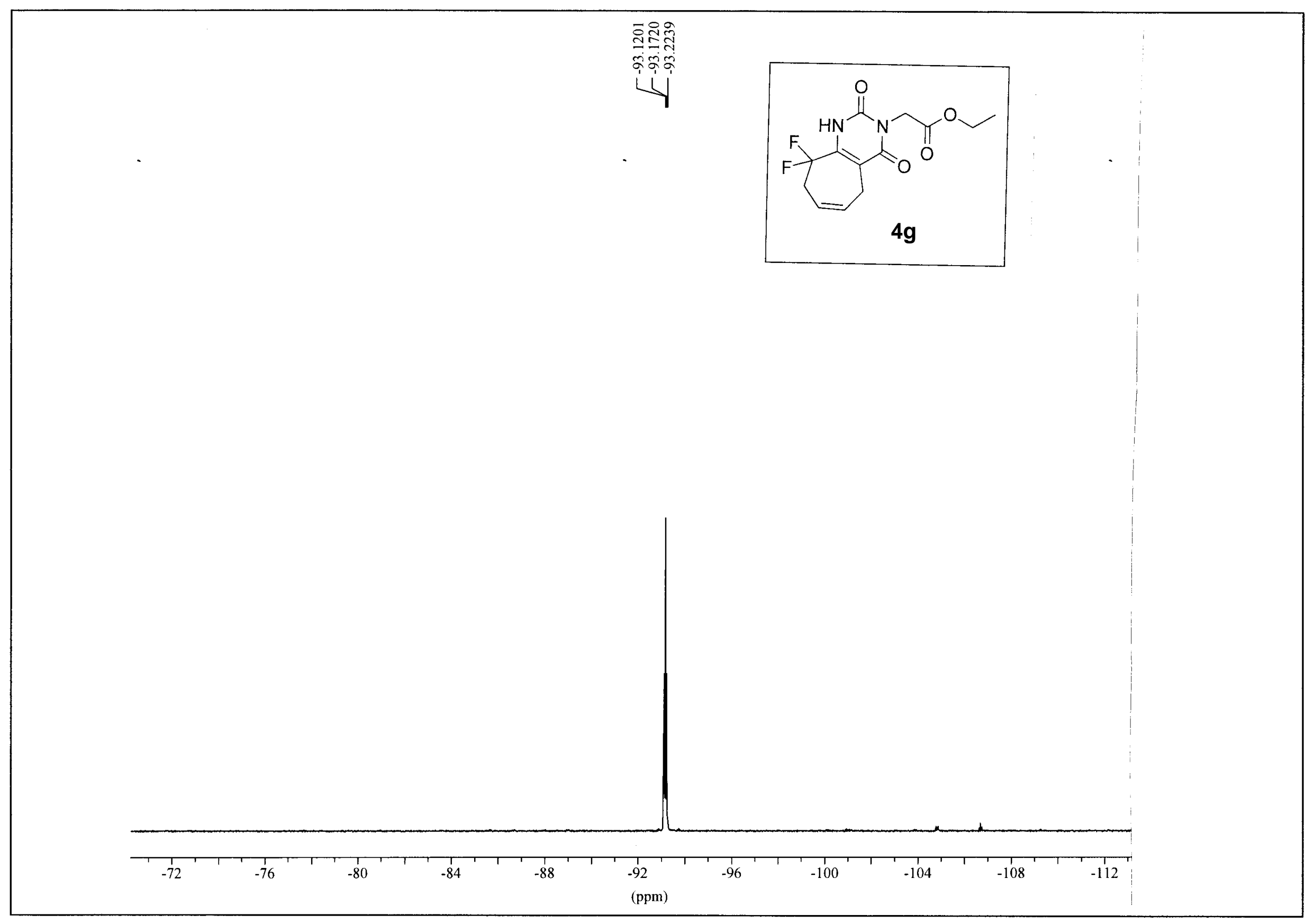




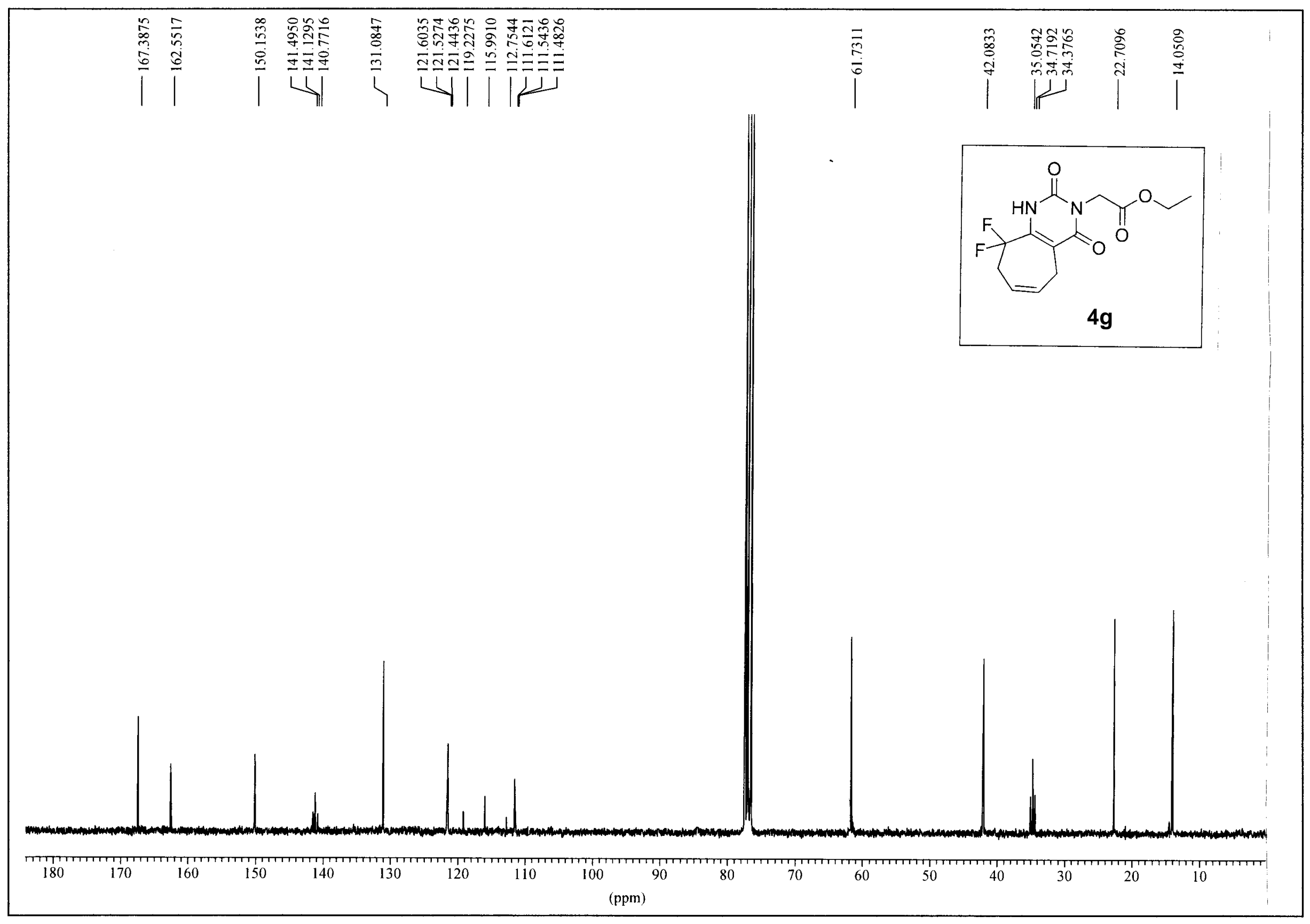




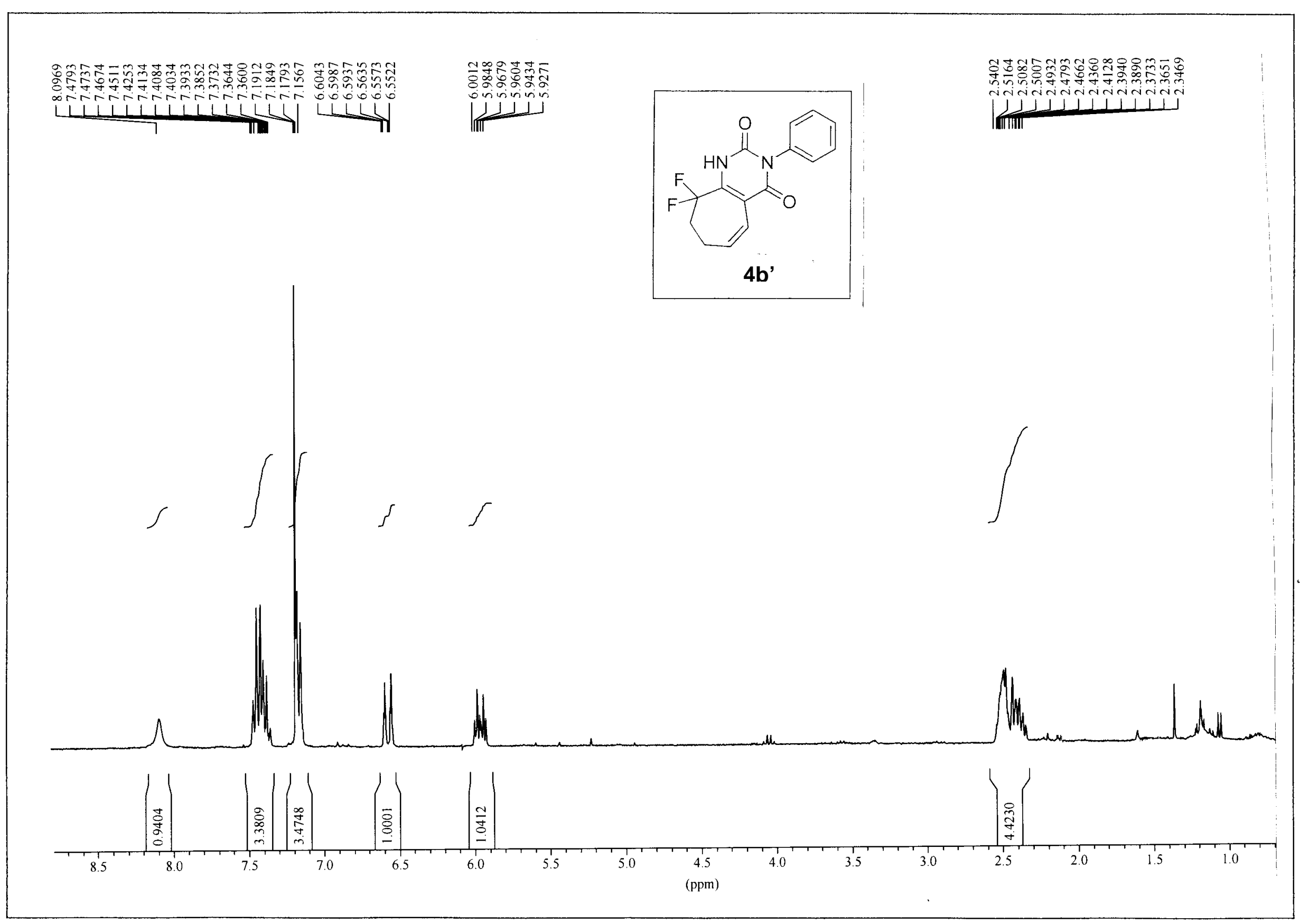




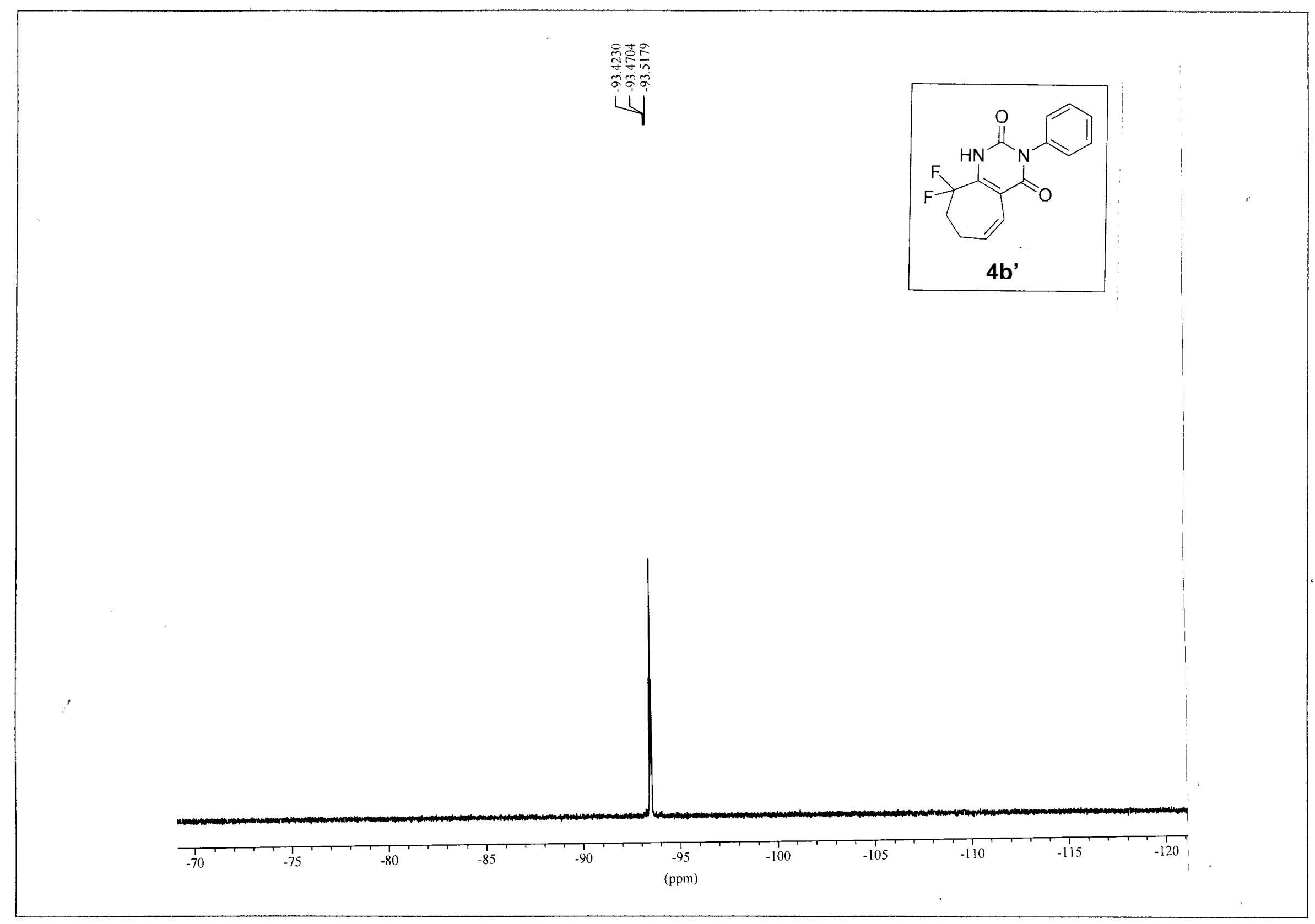




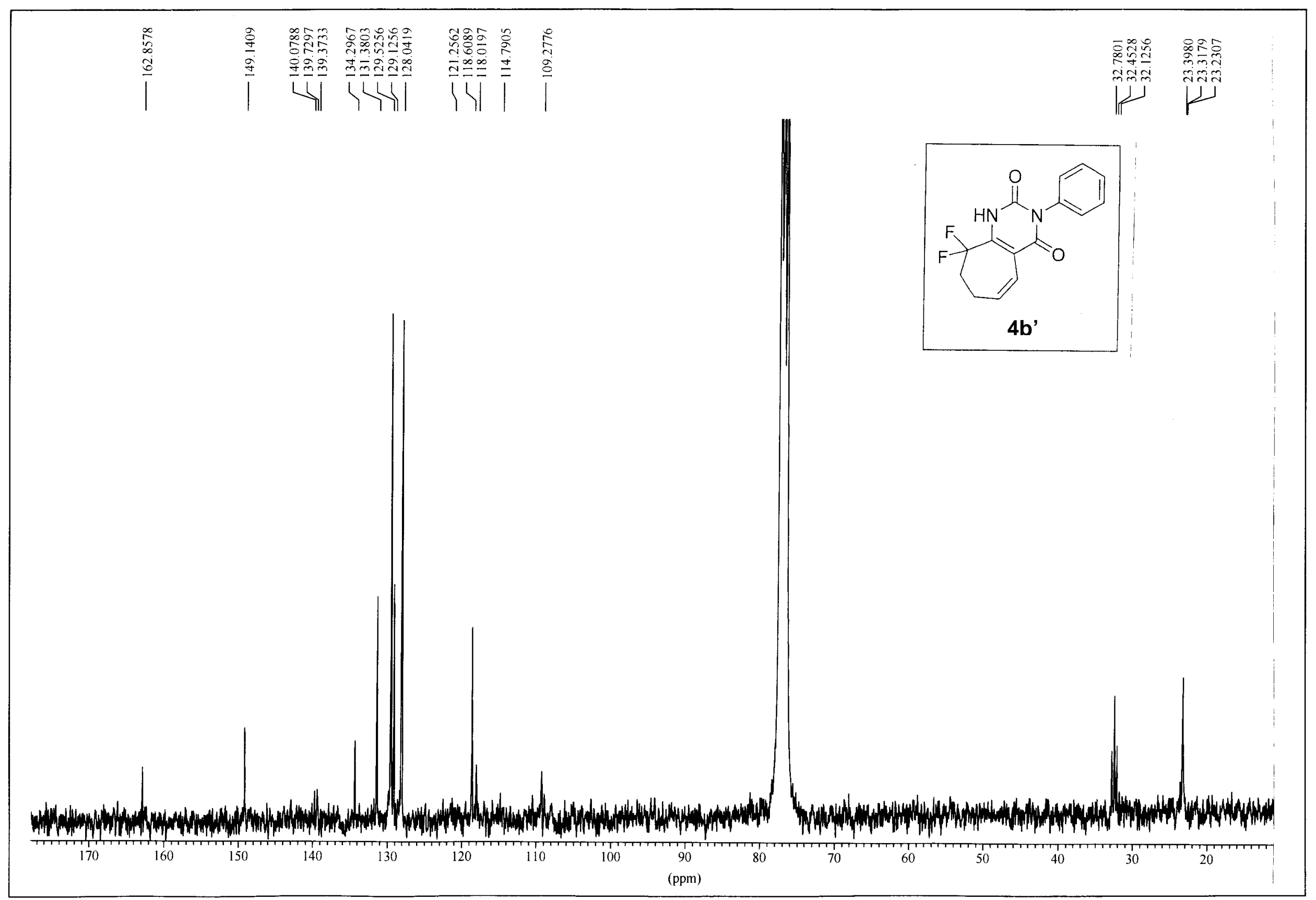

S88 


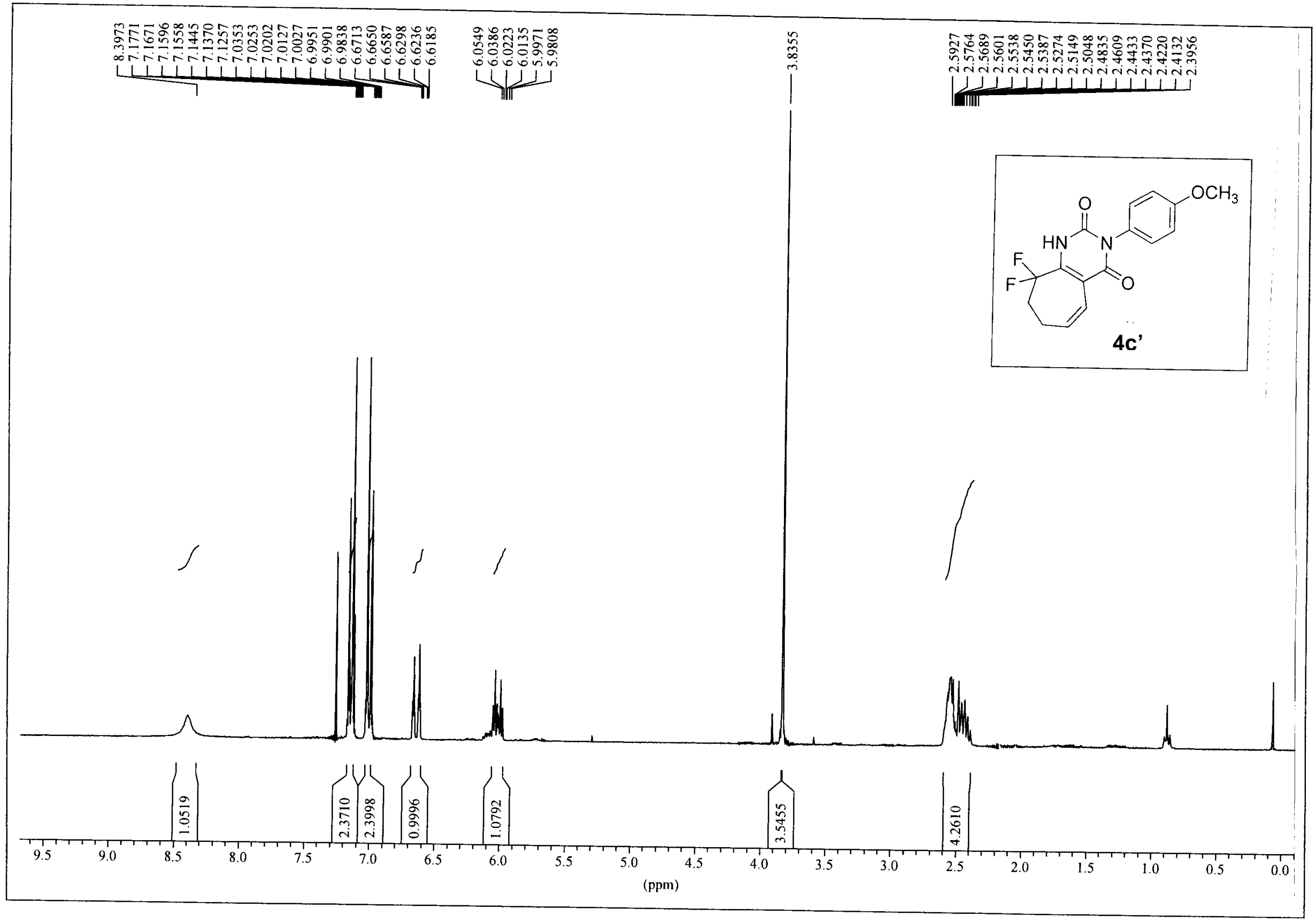




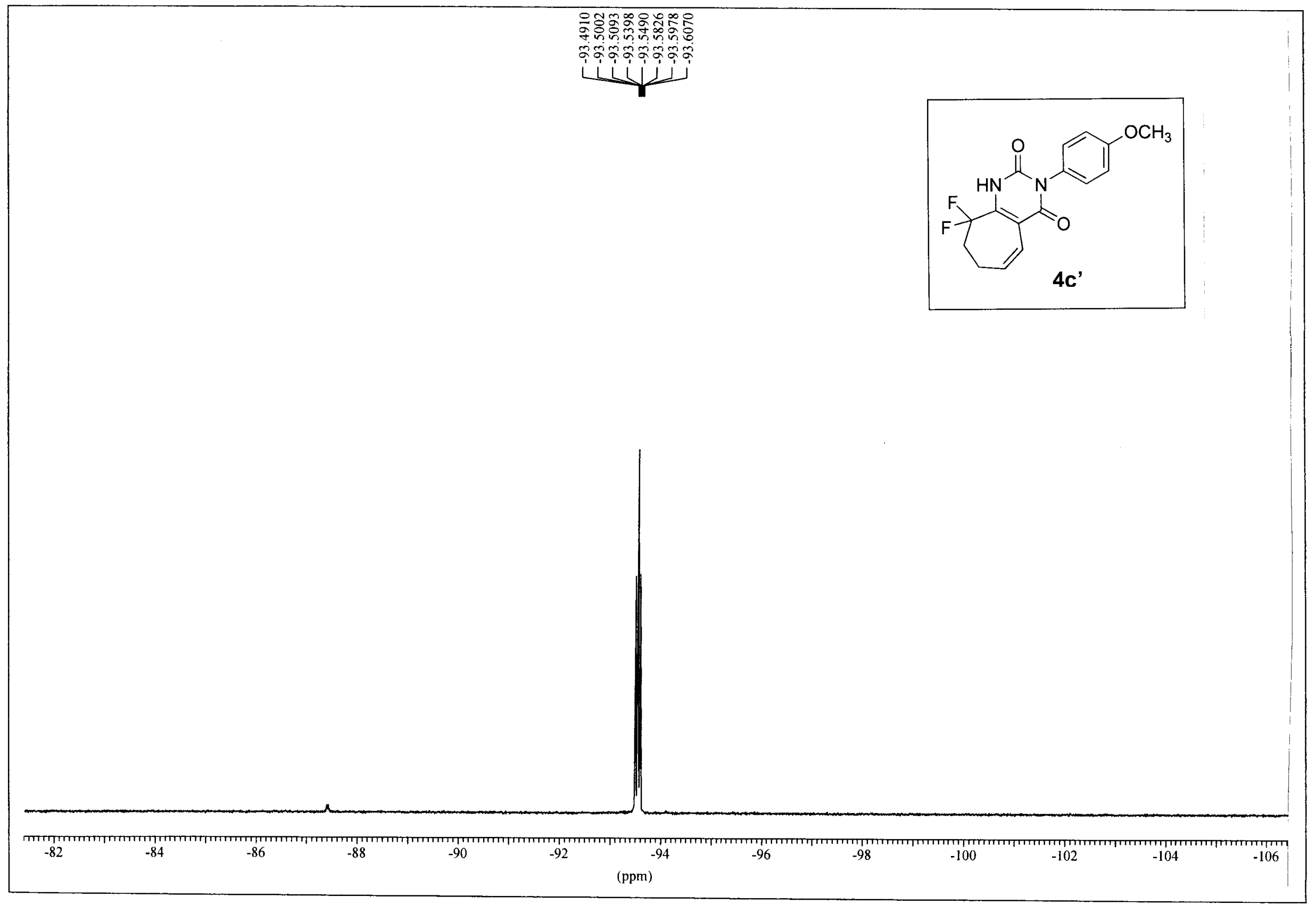




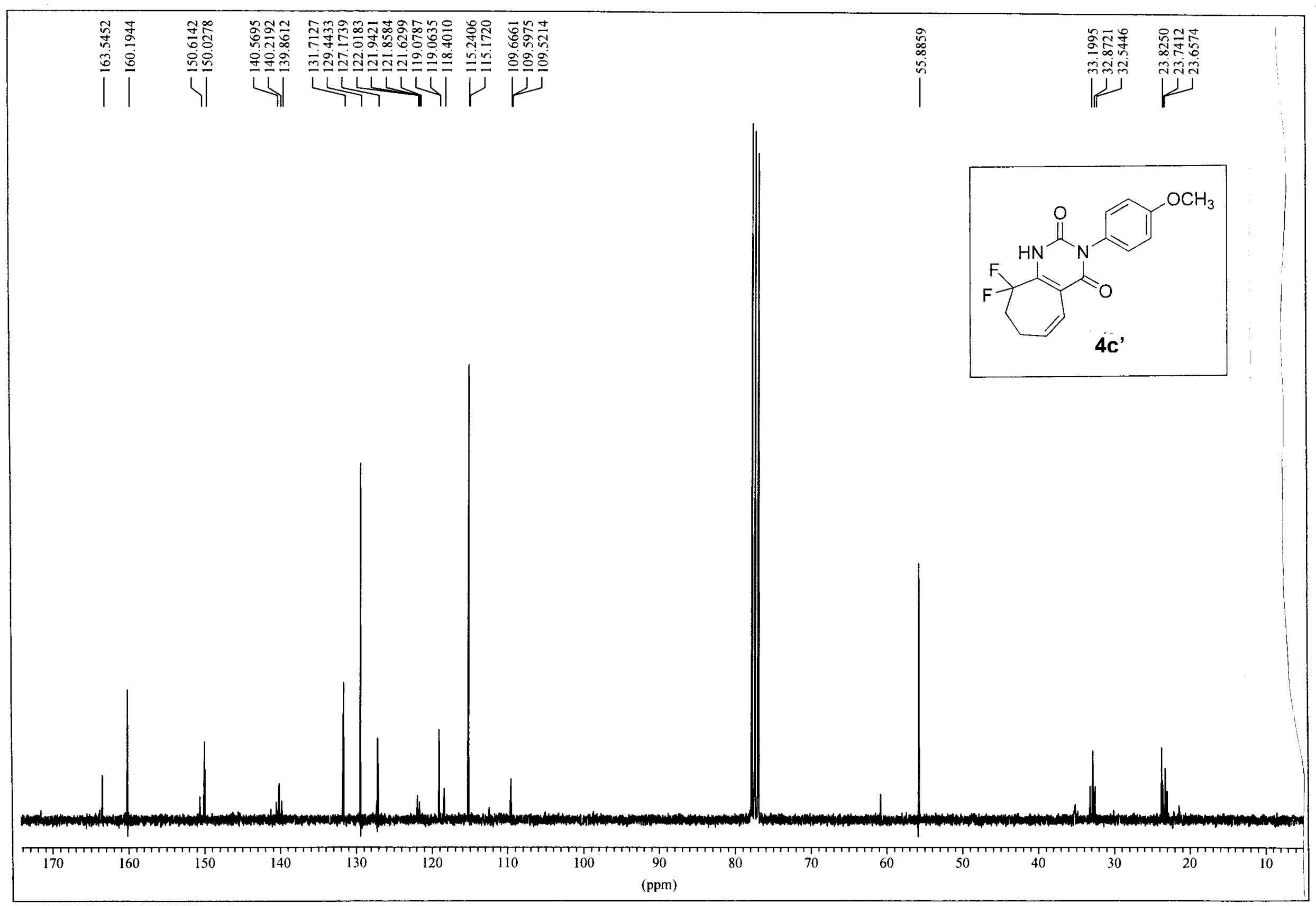




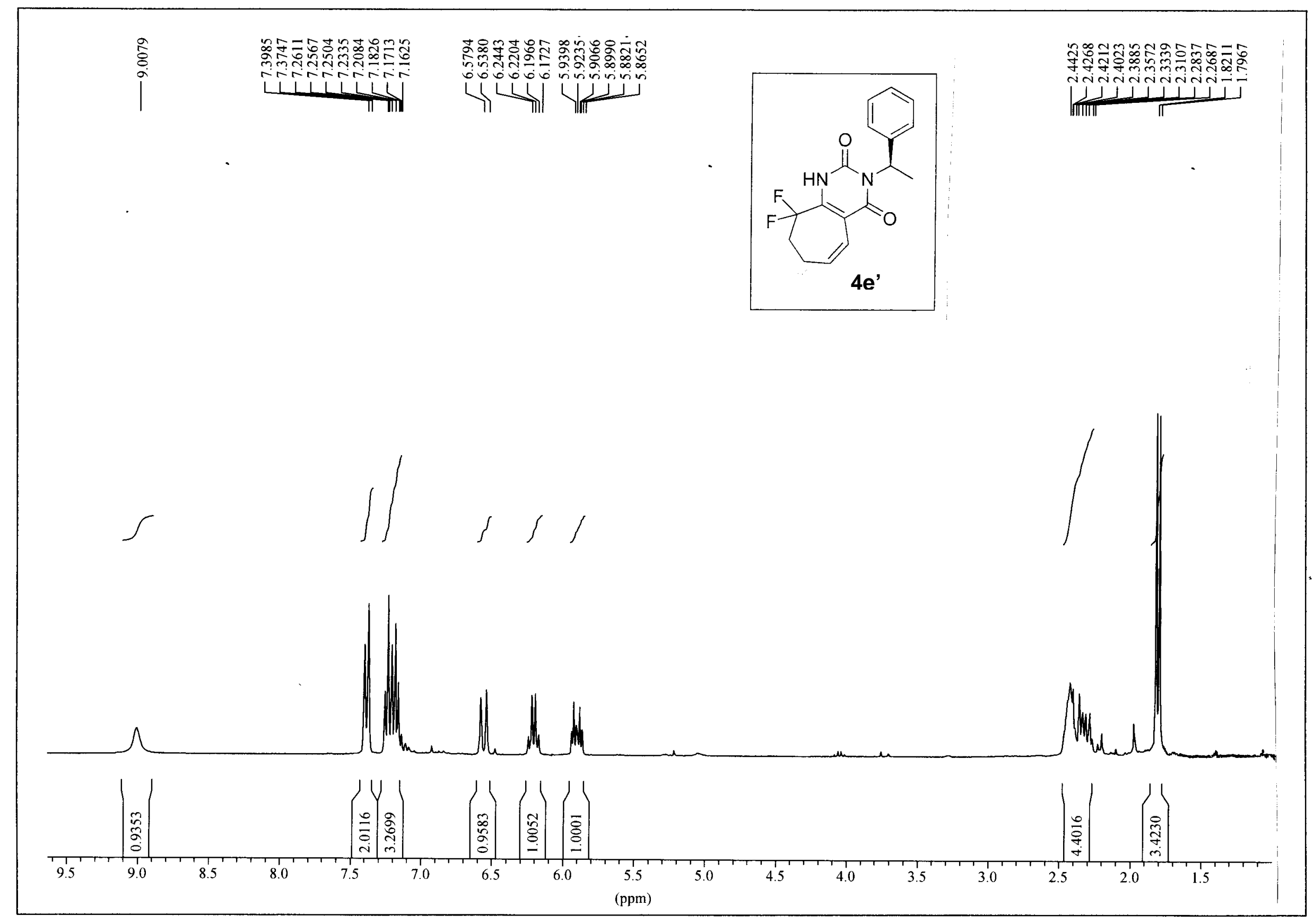




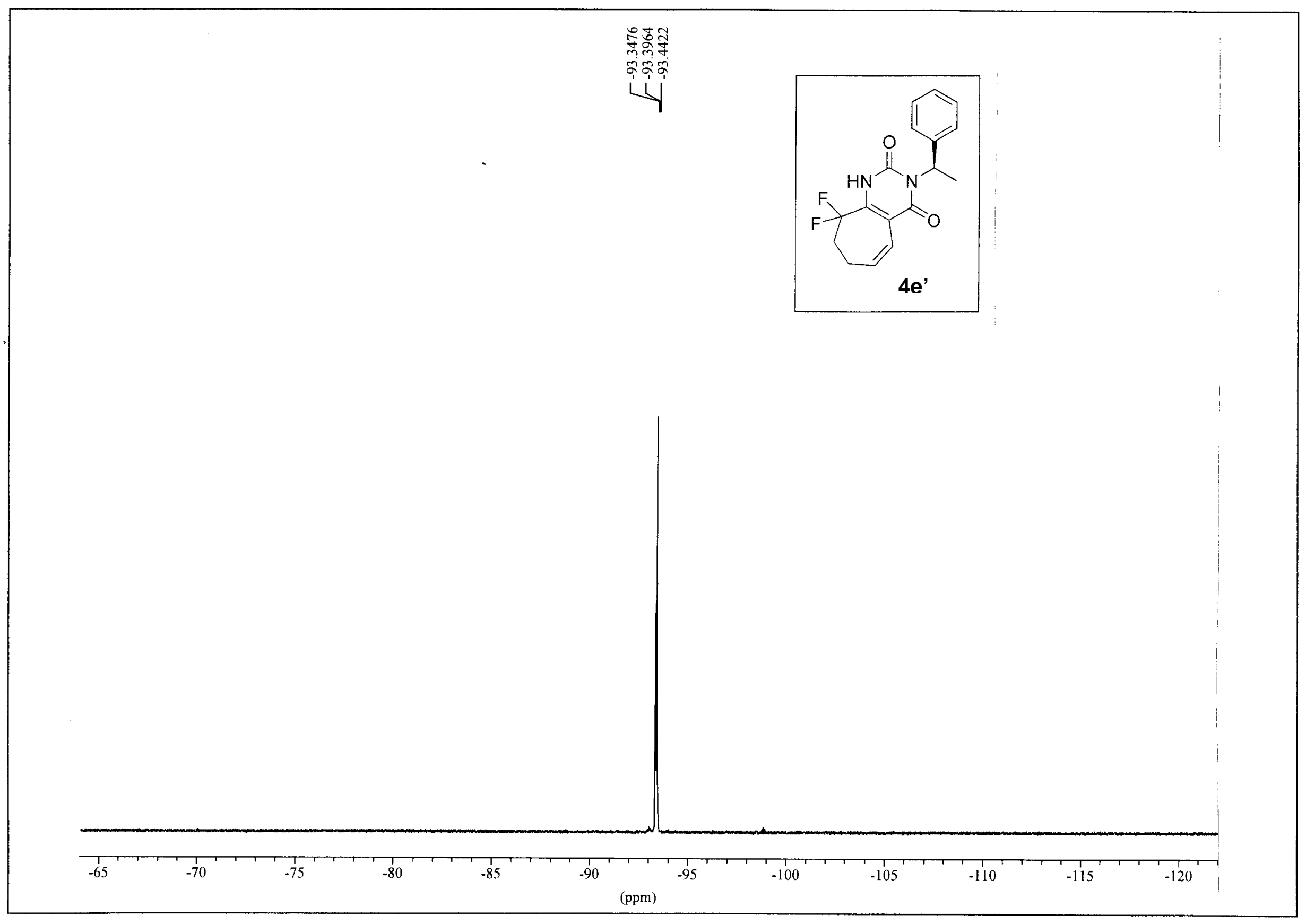




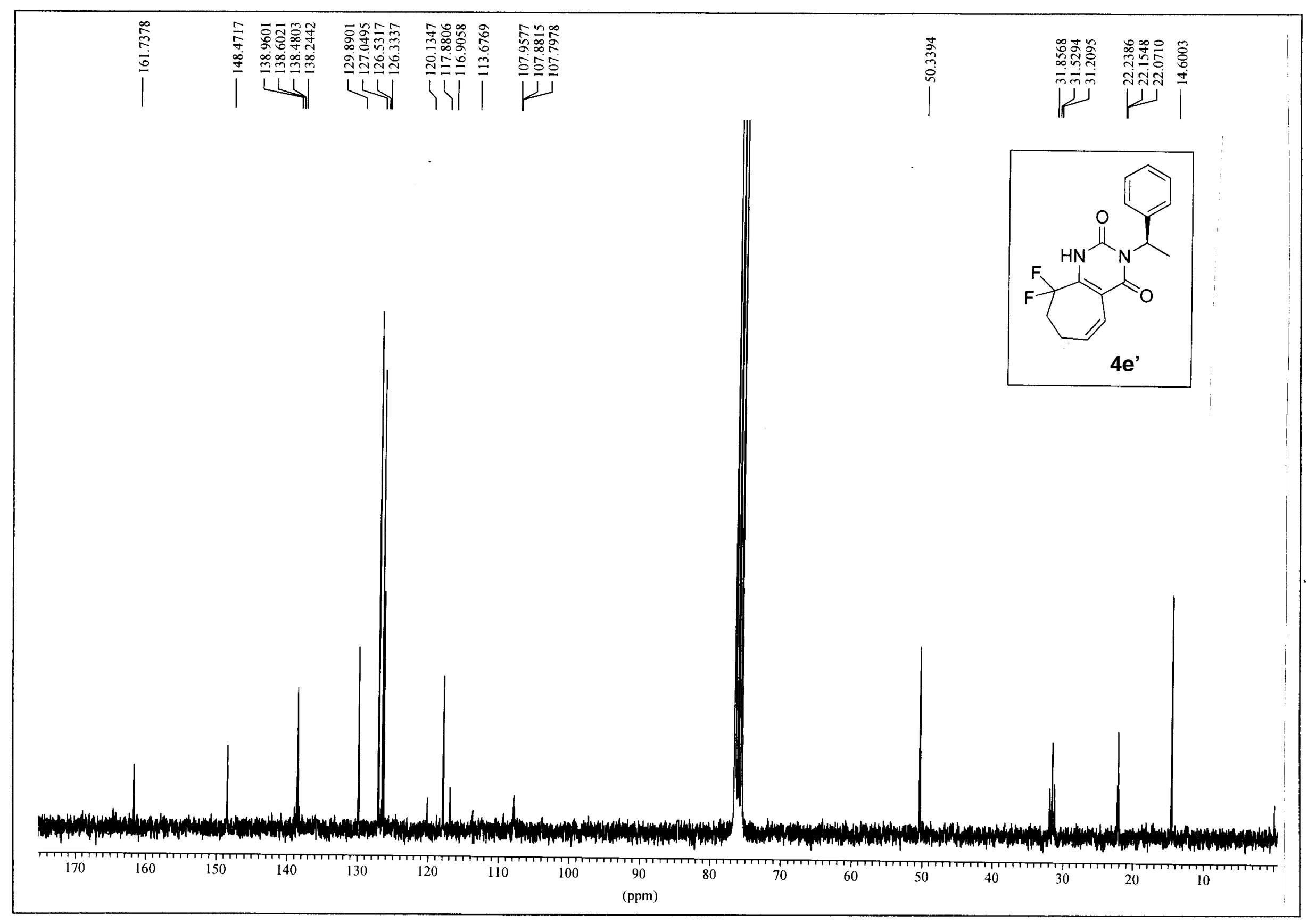




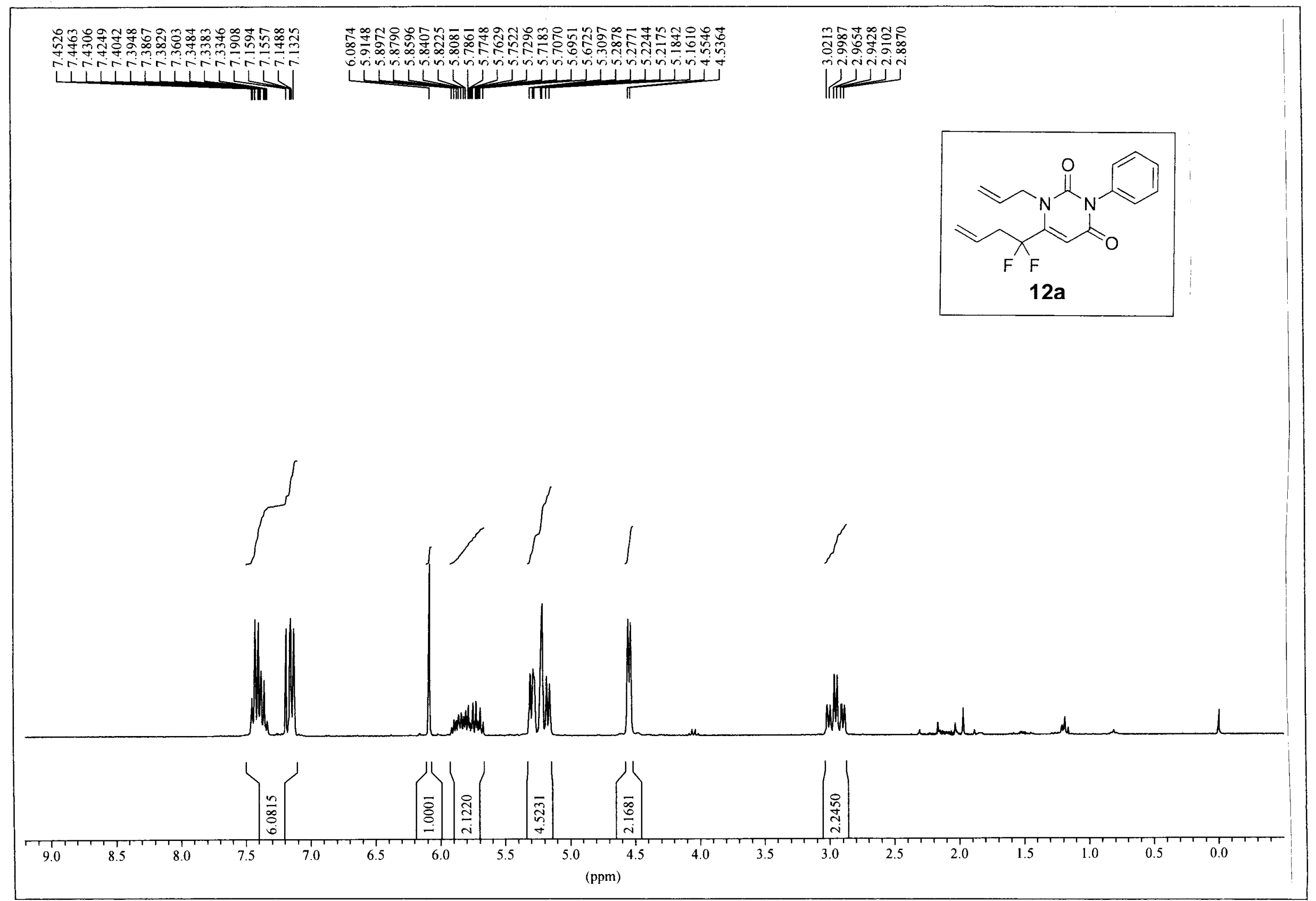




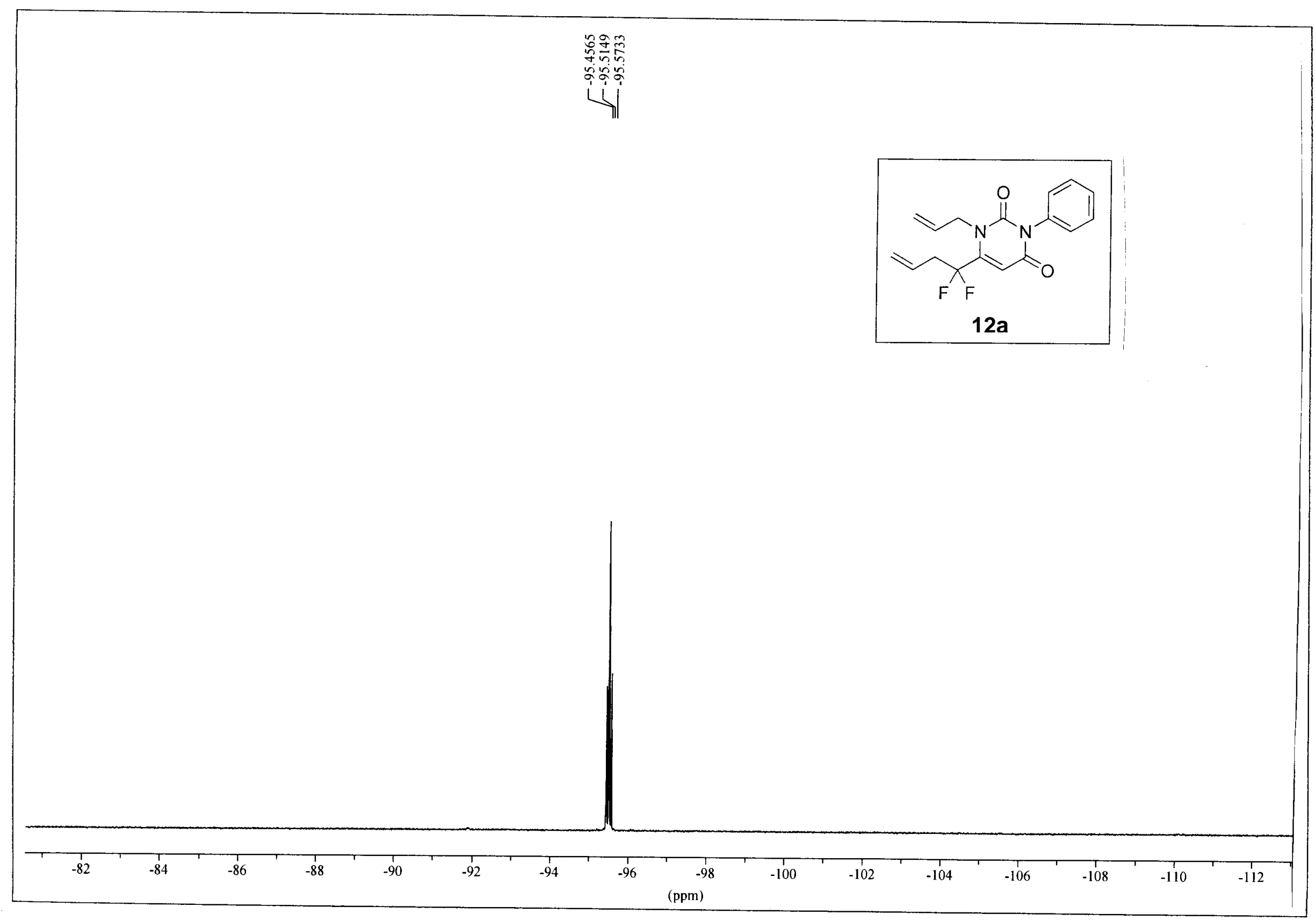




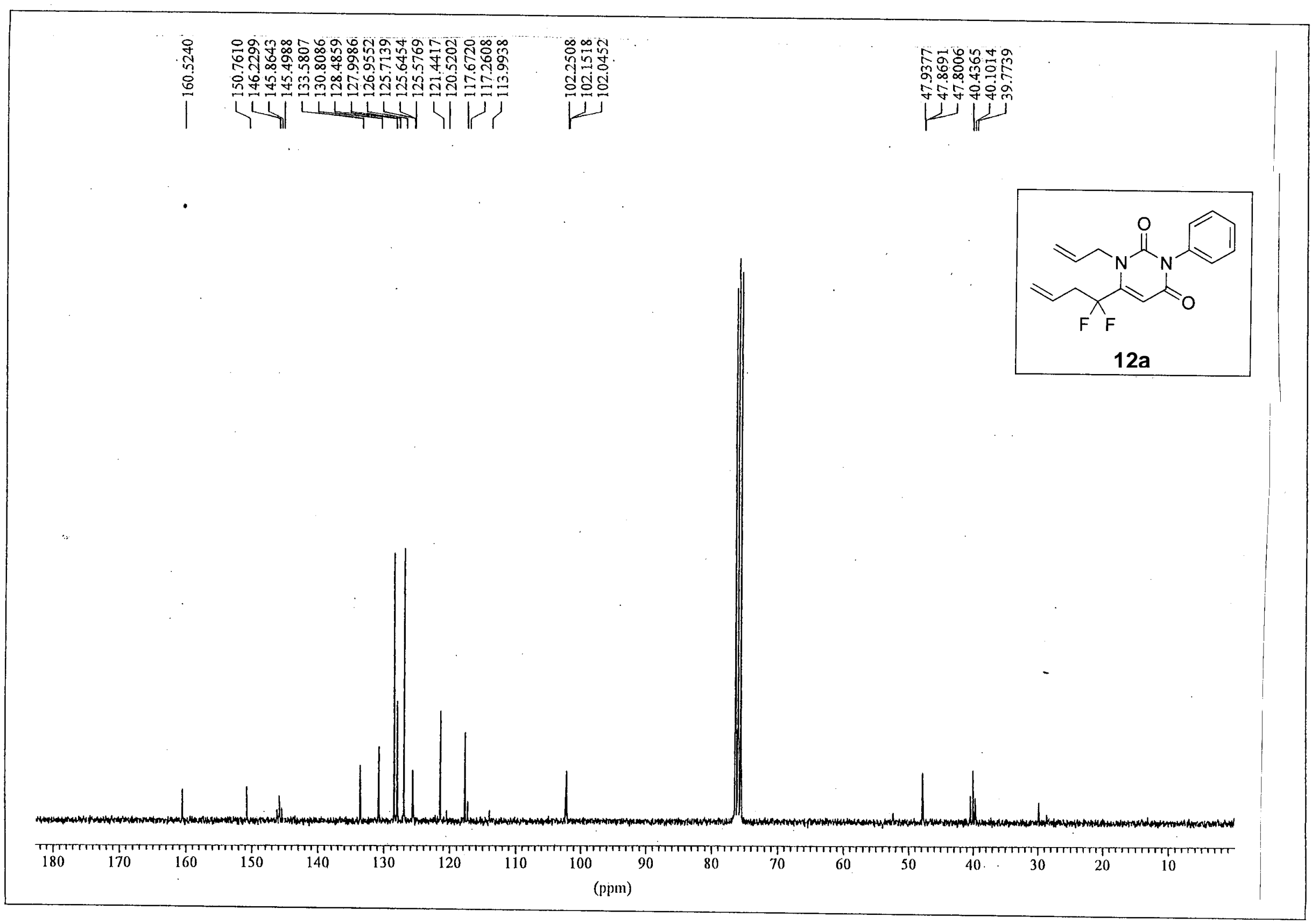




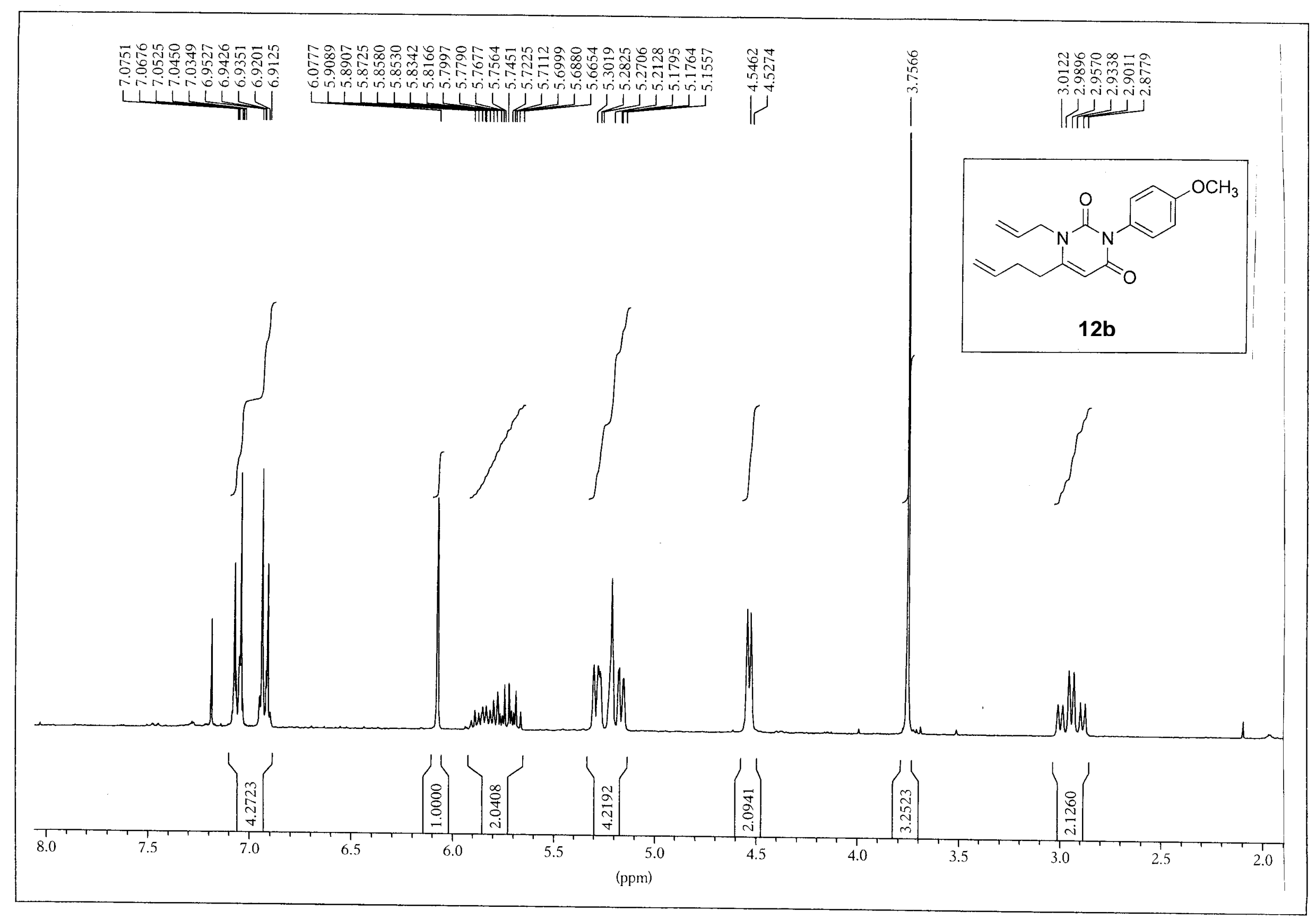




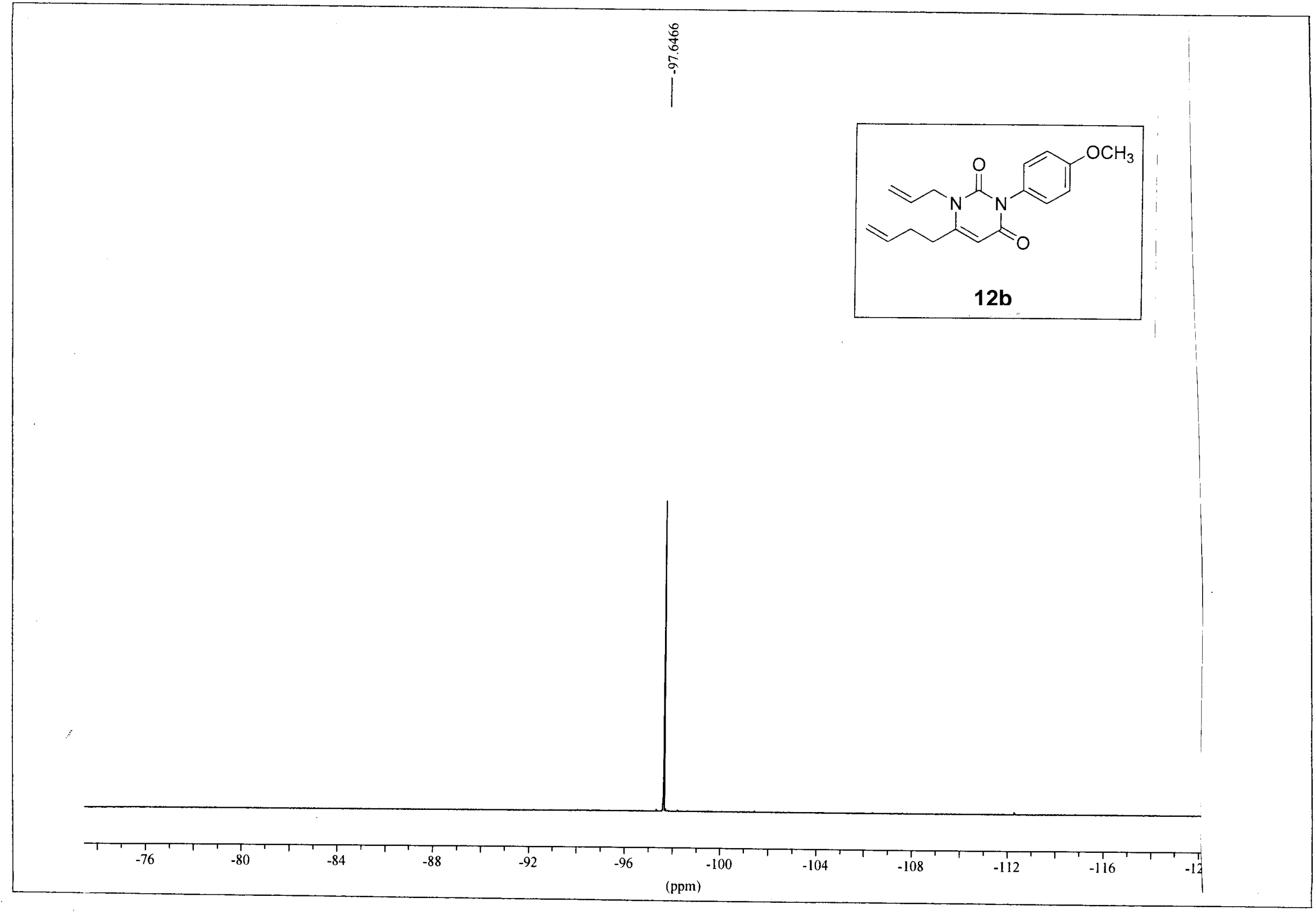




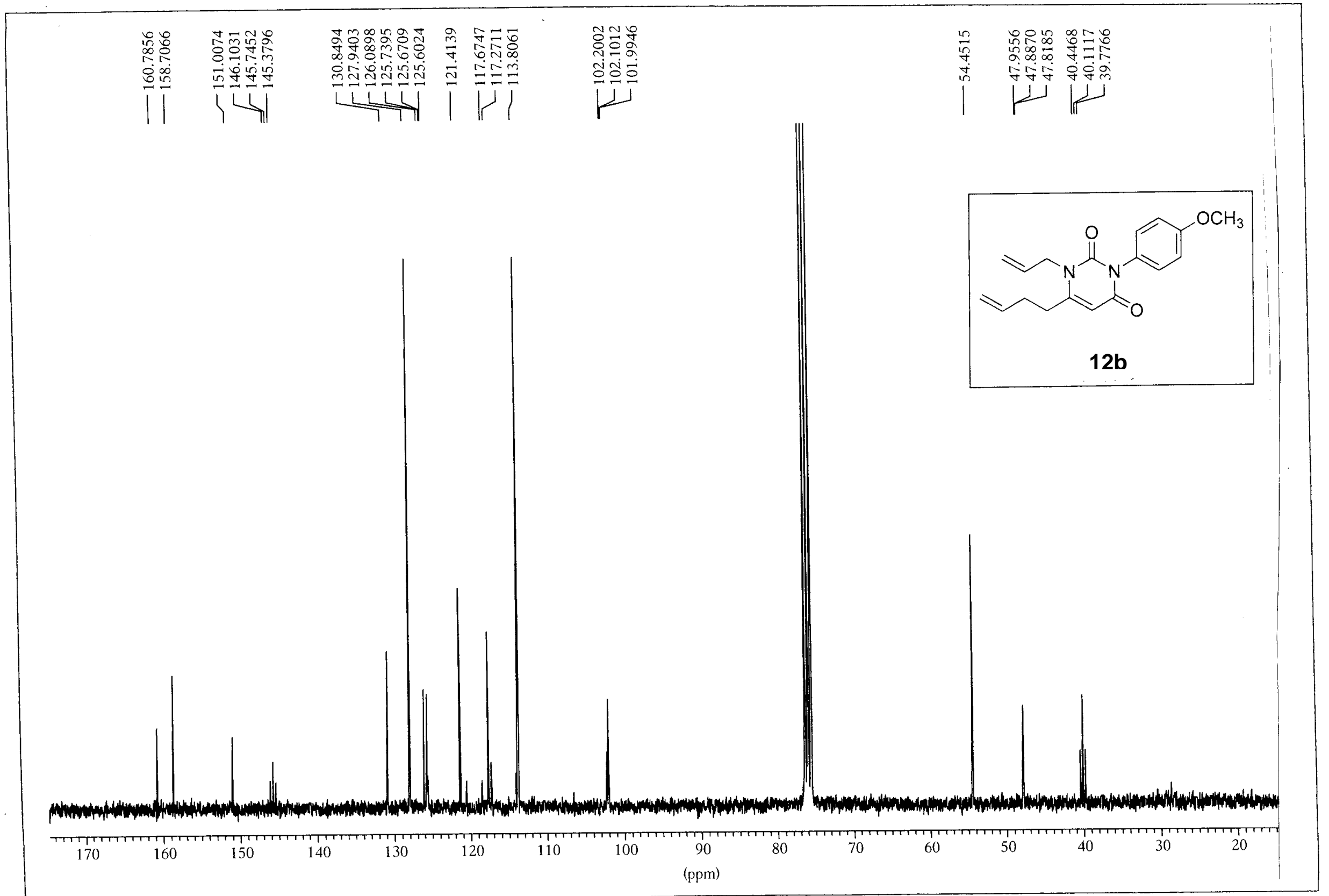




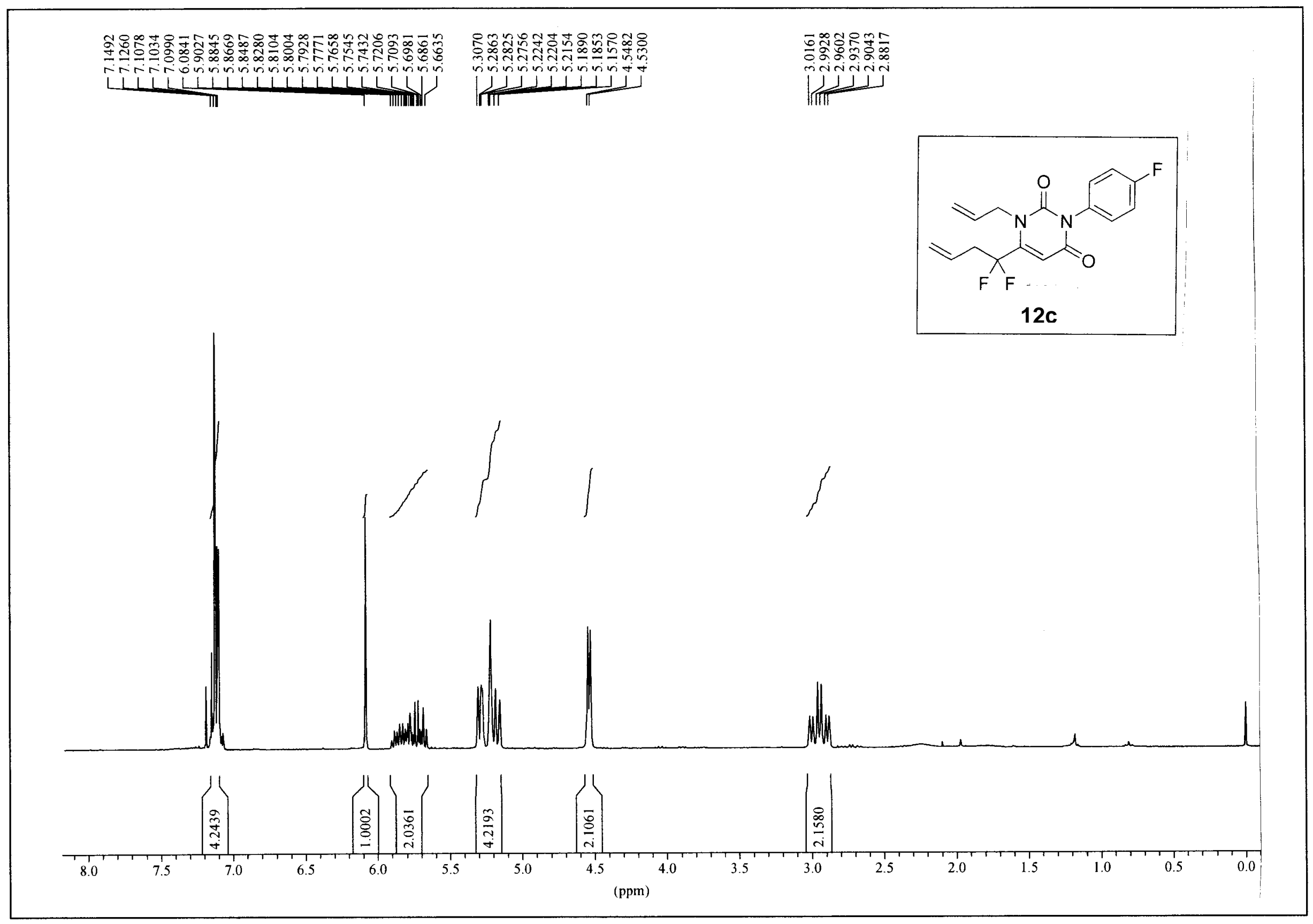




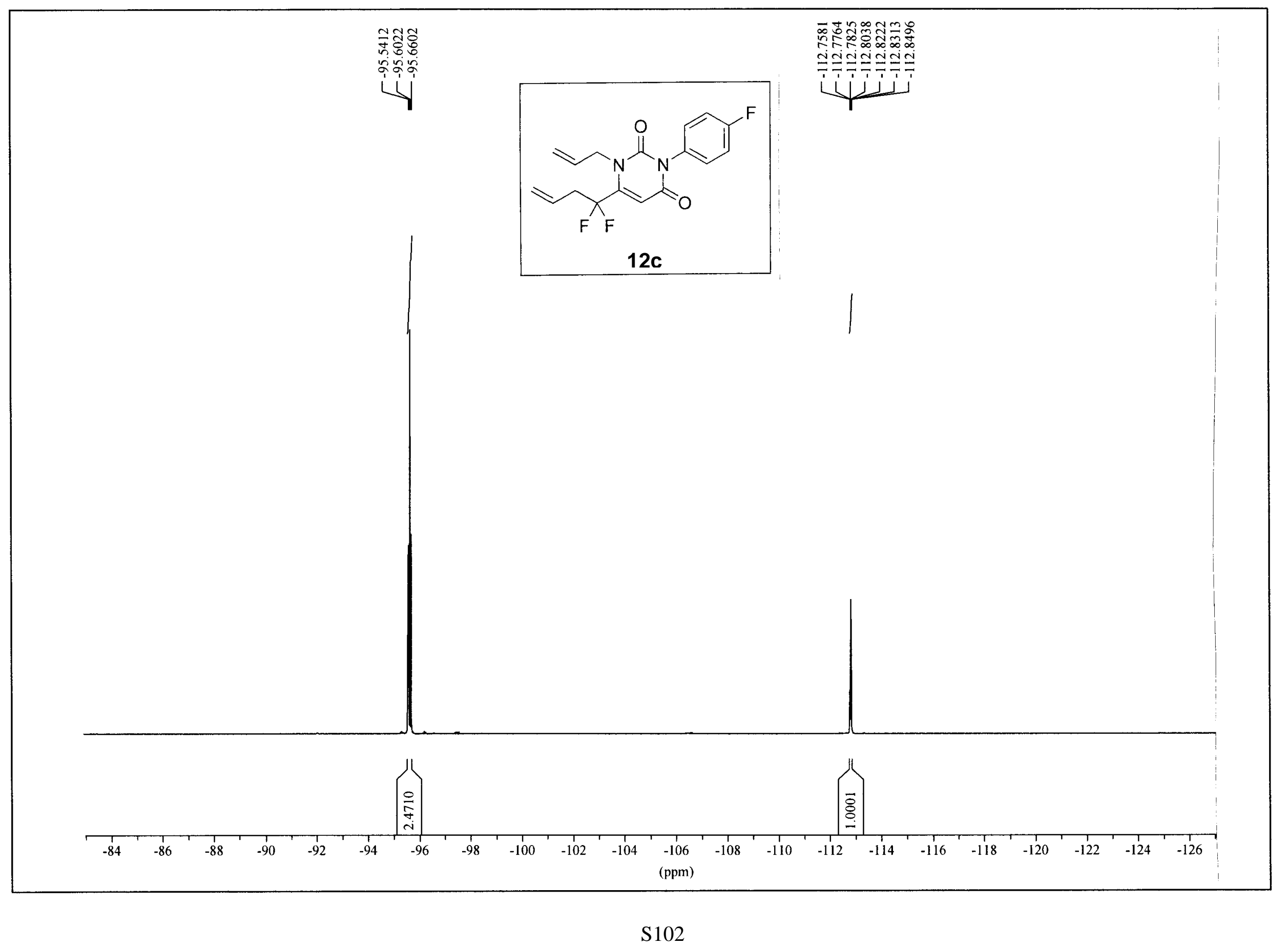




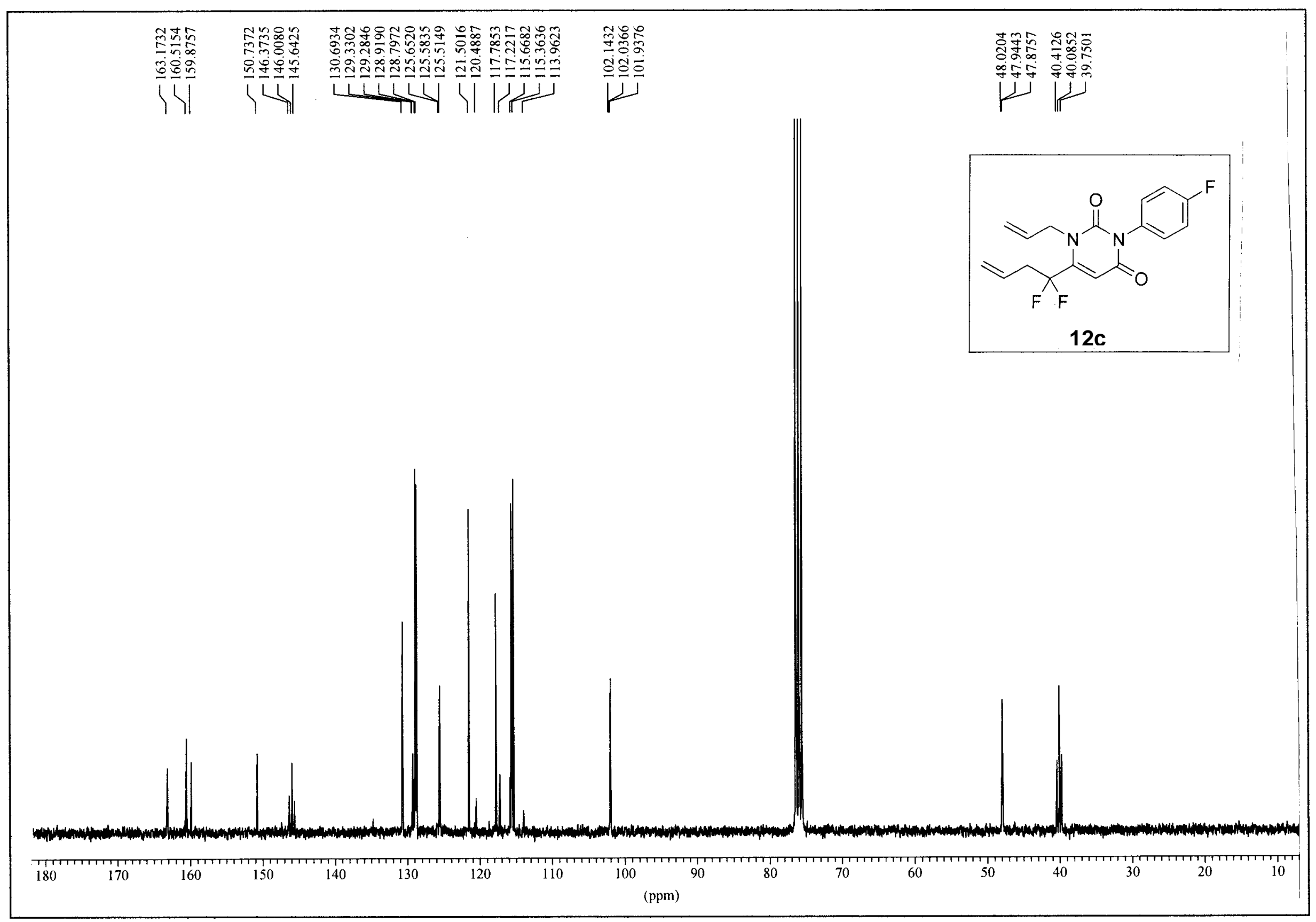




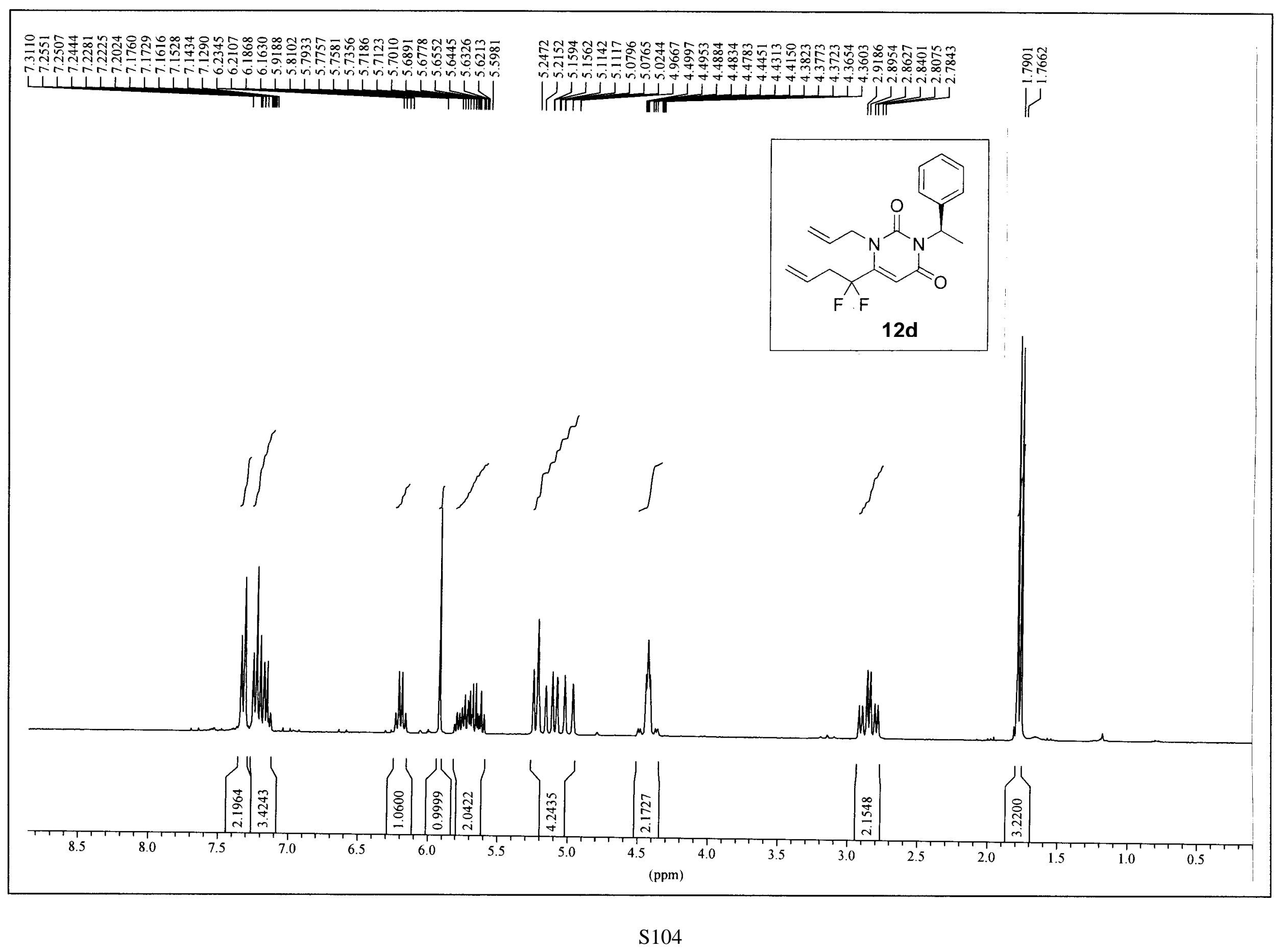




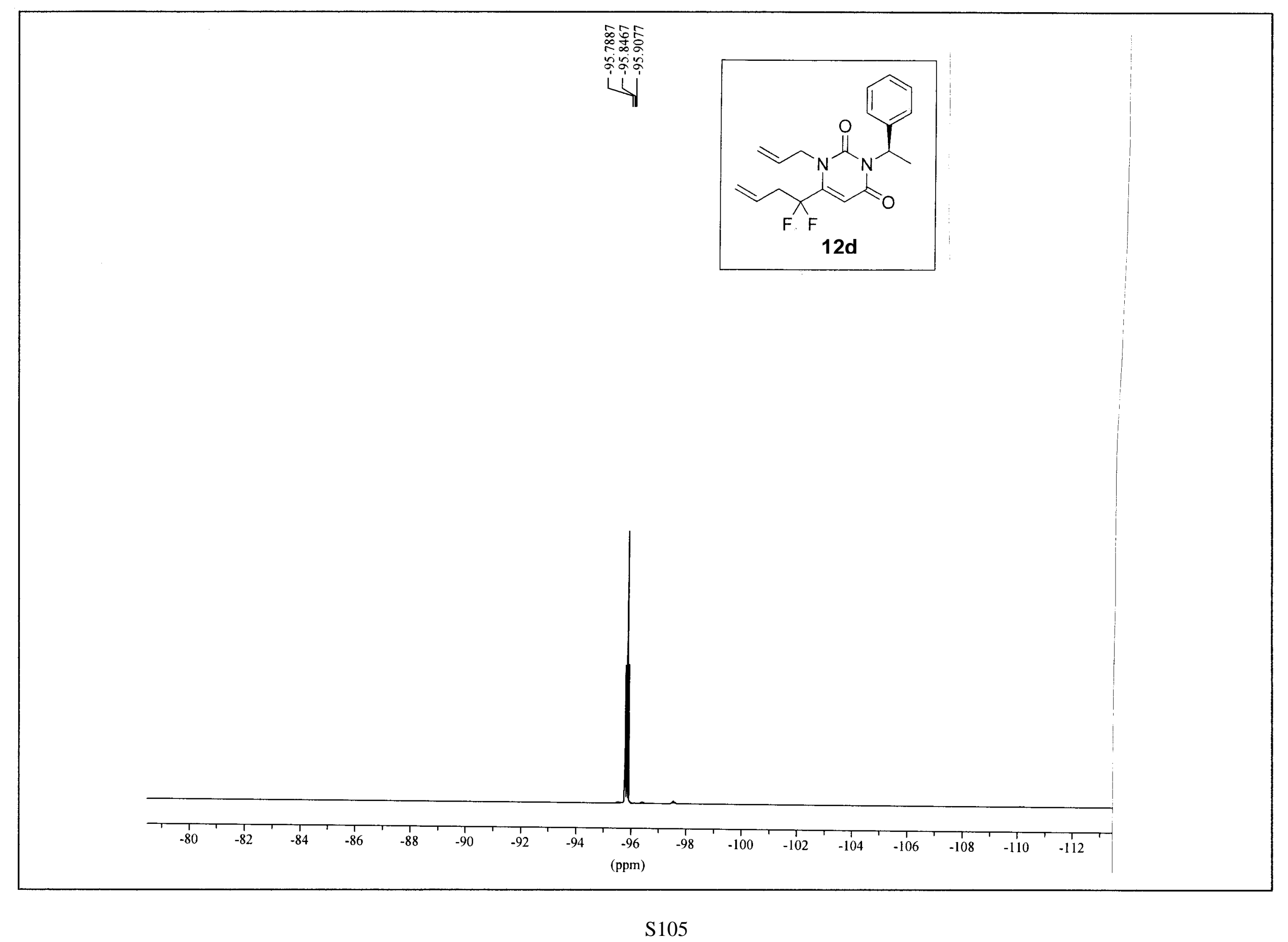




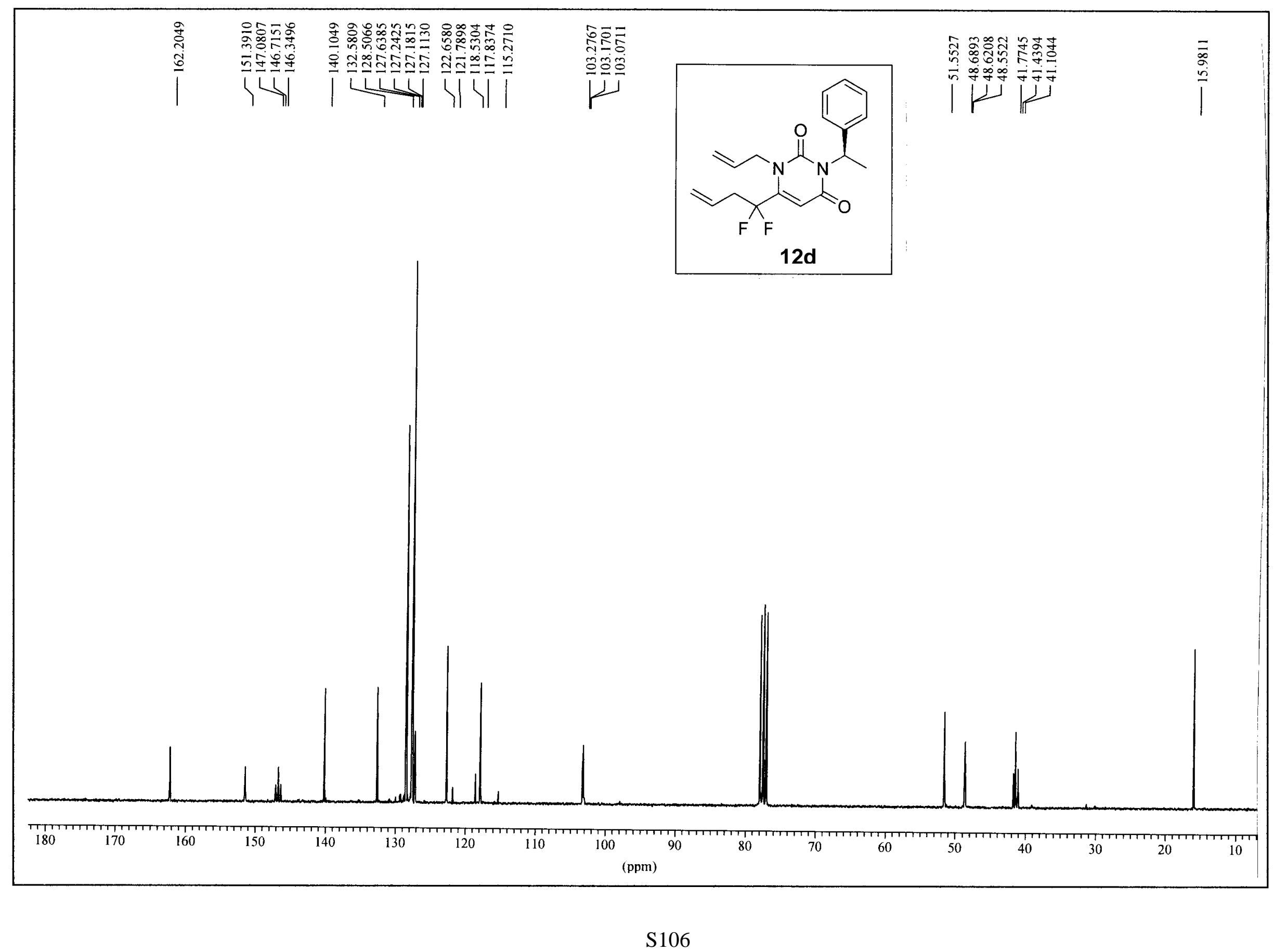




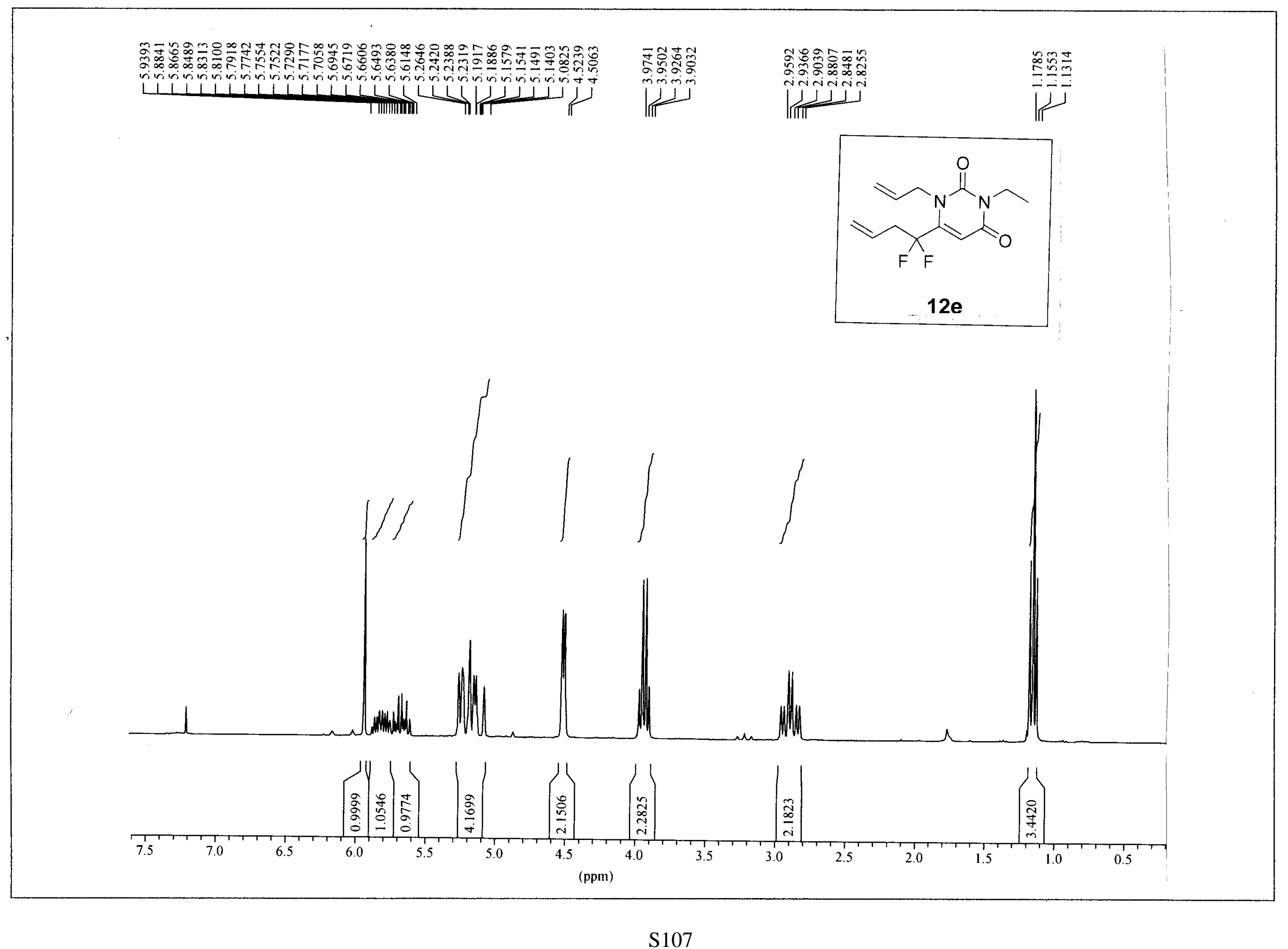




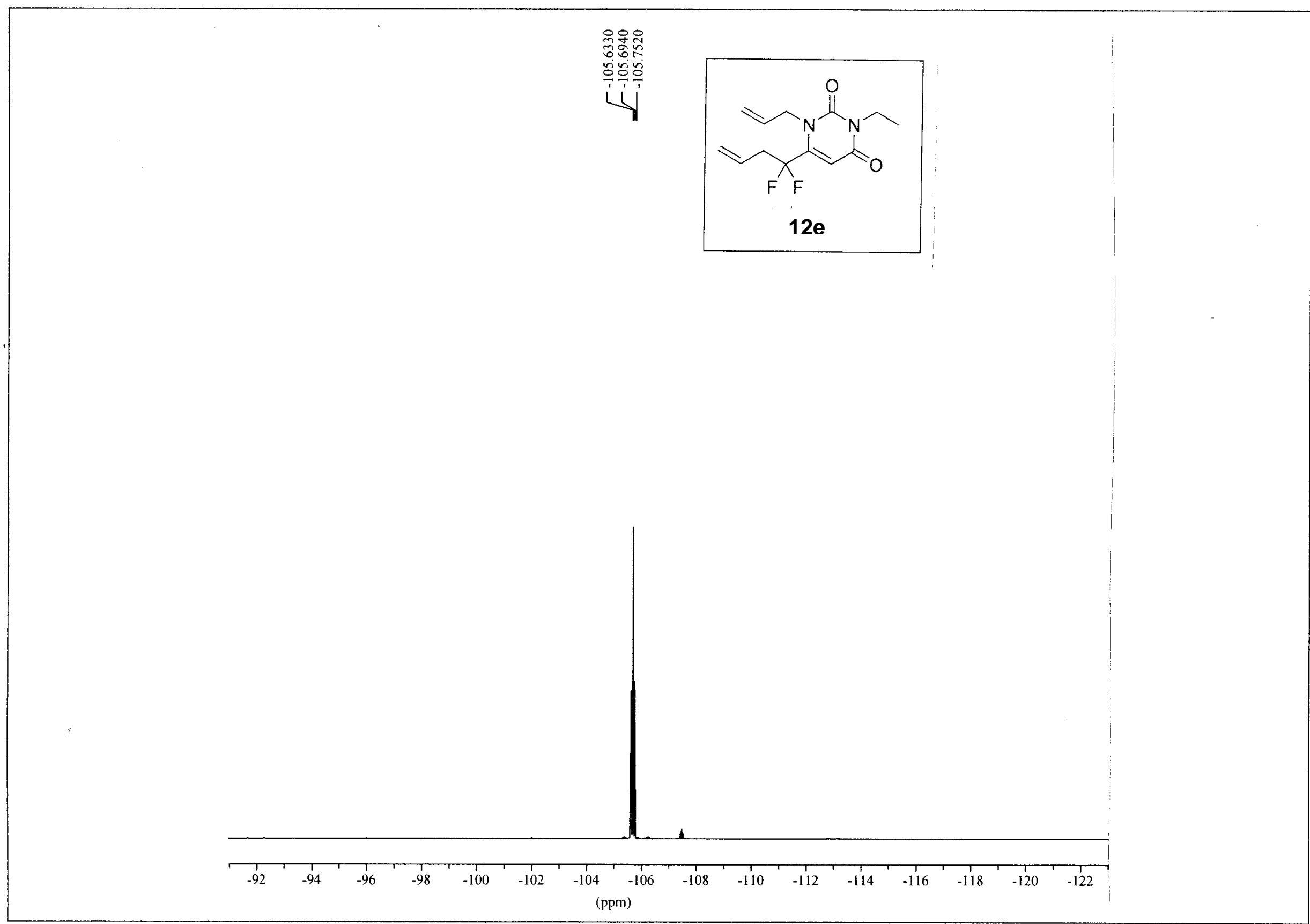




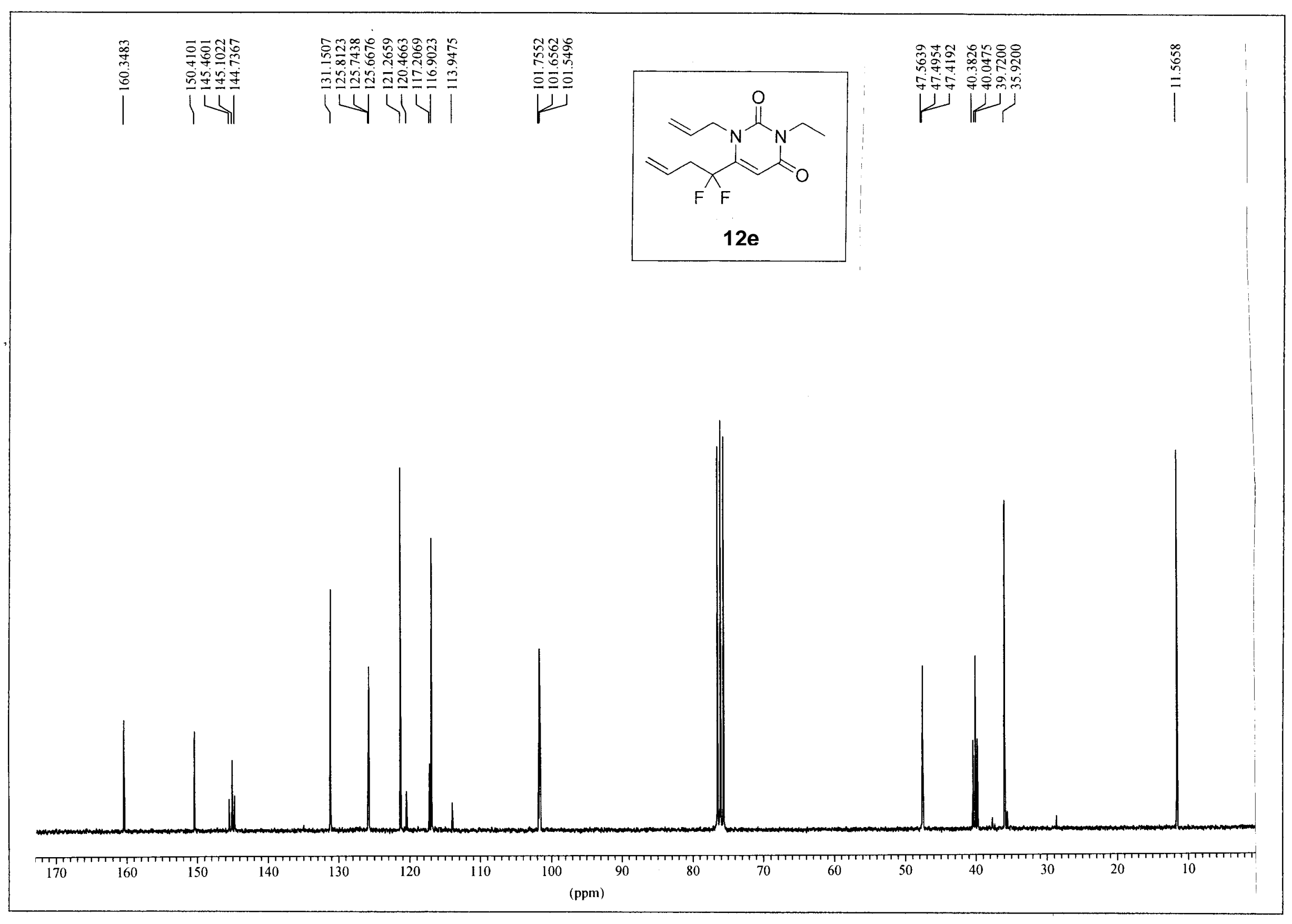




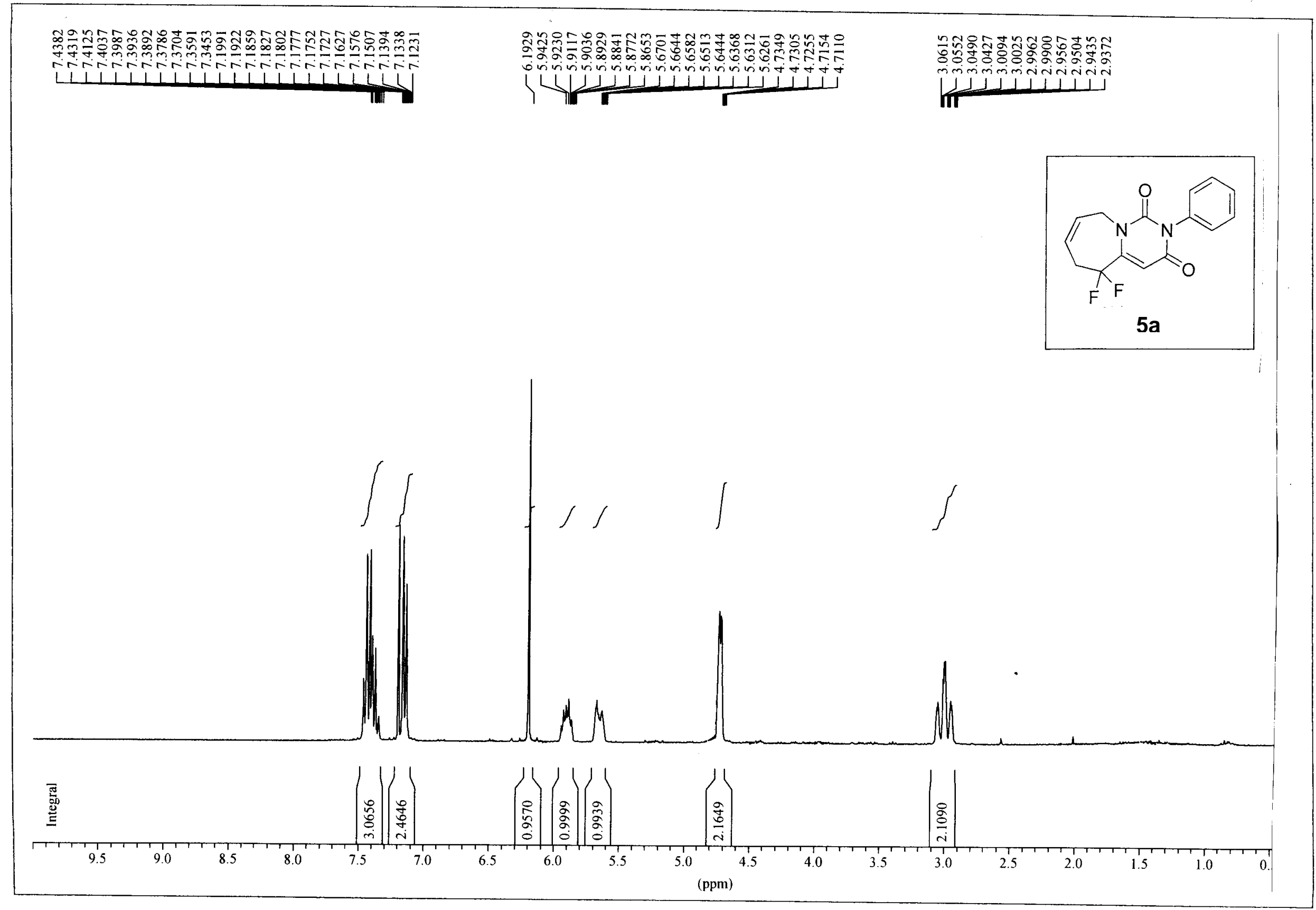




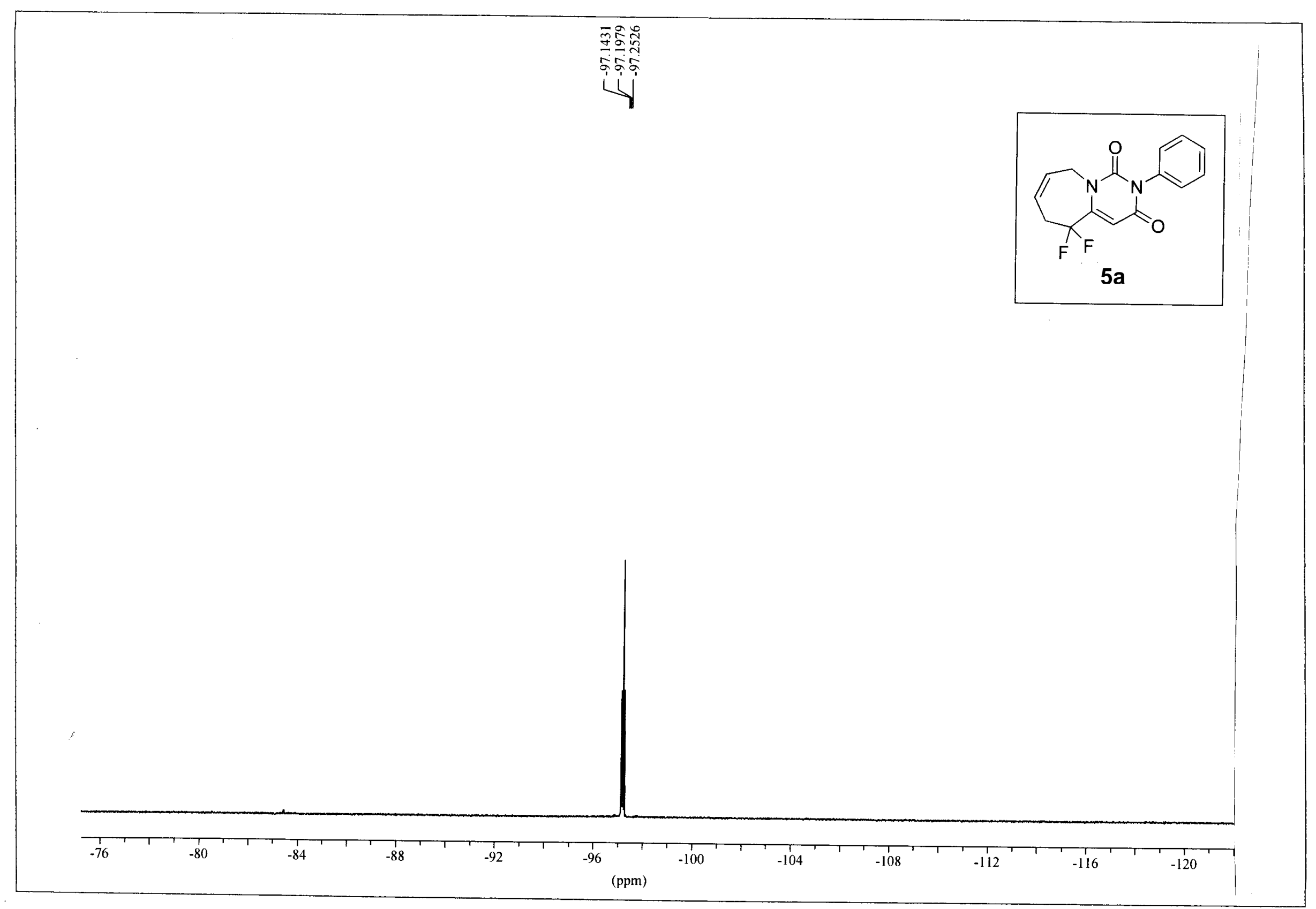




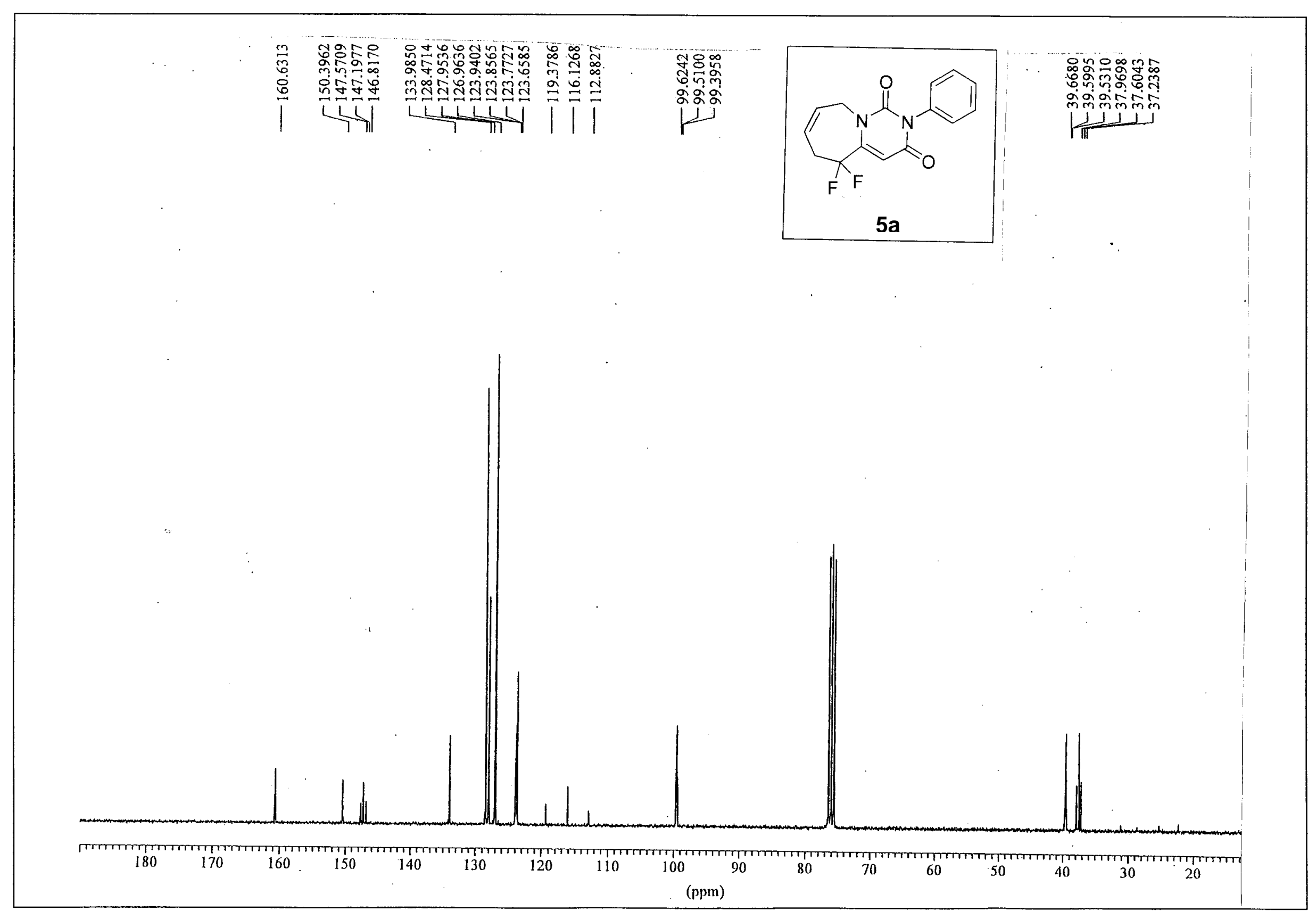




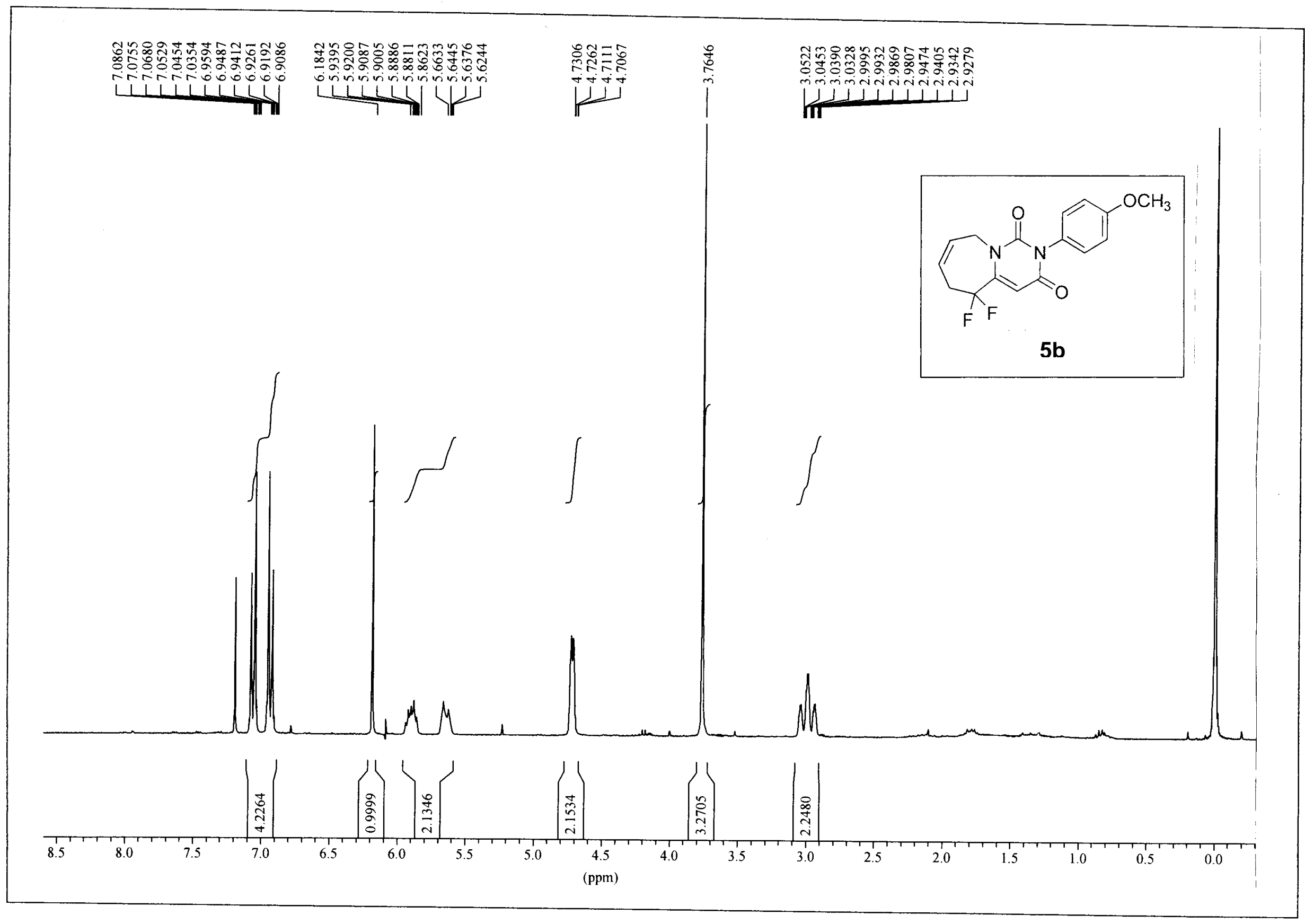




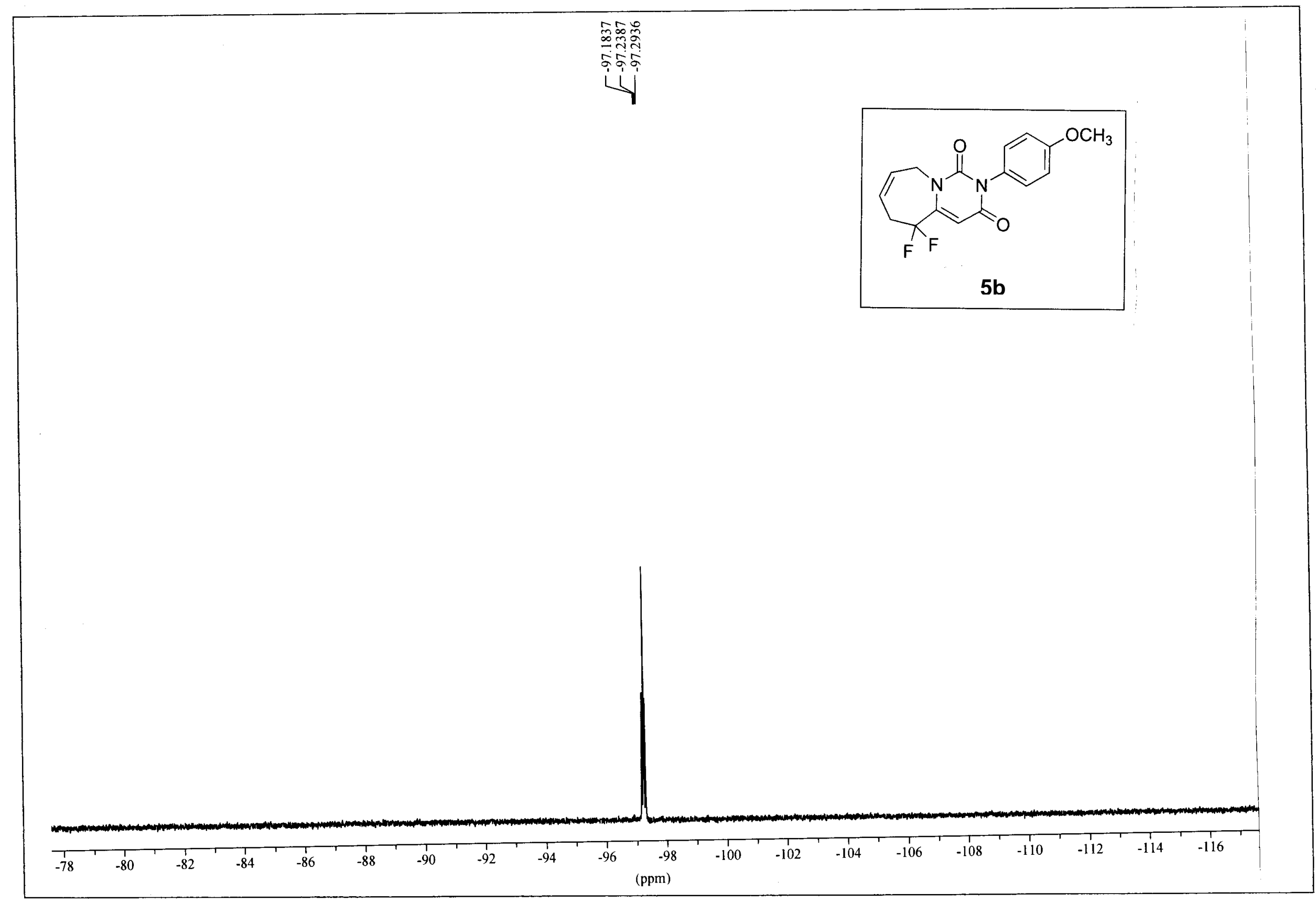




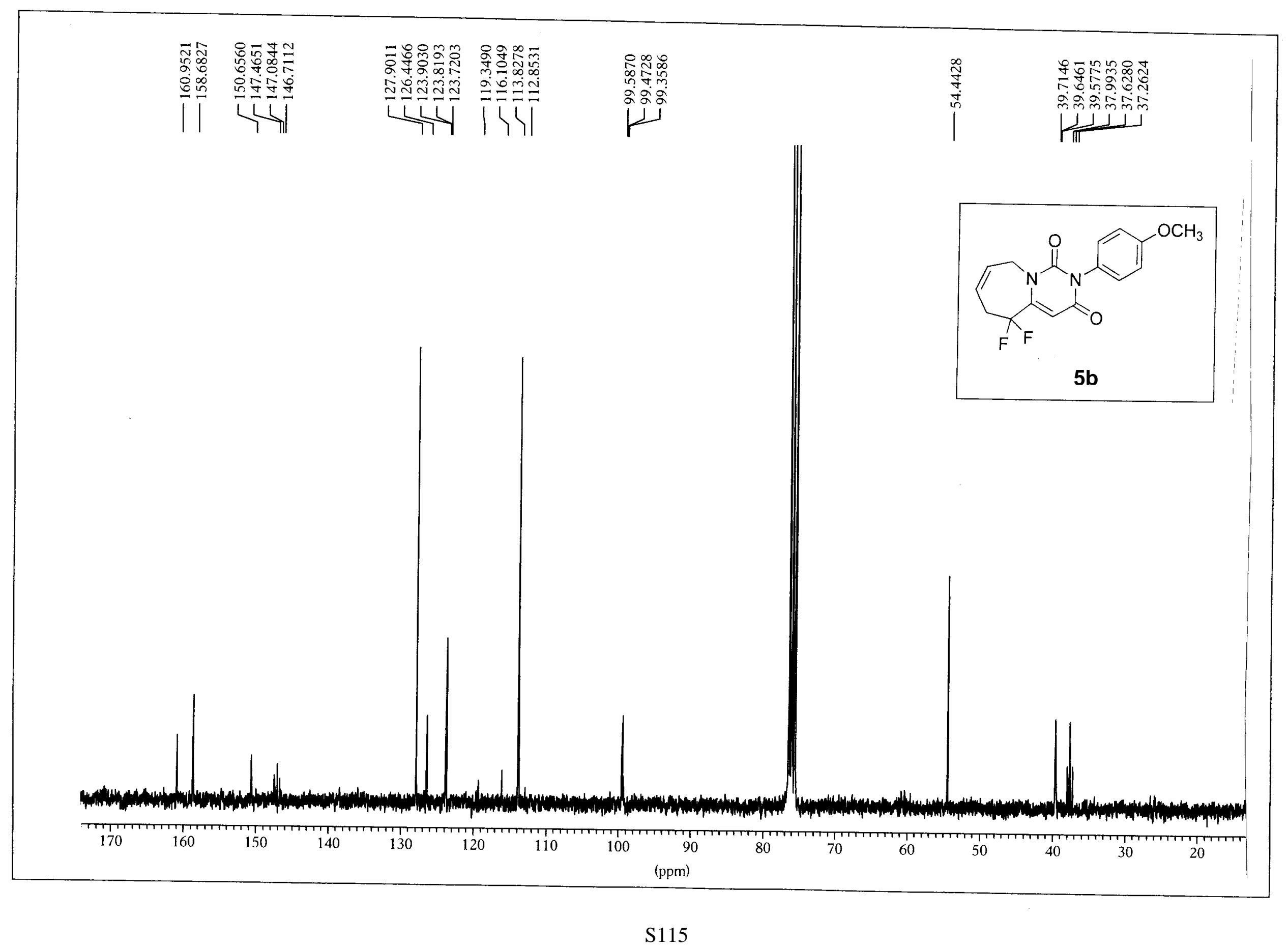




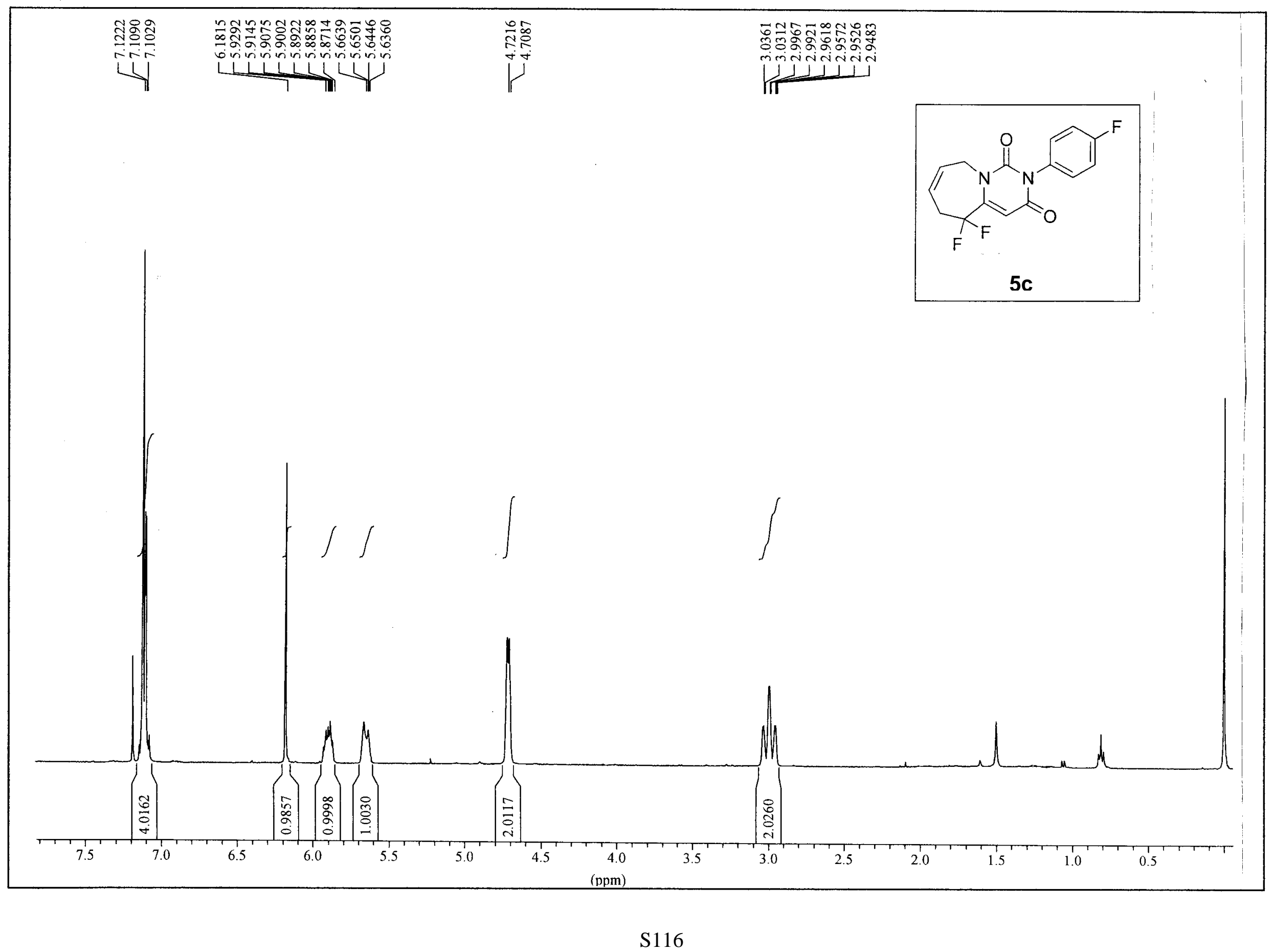




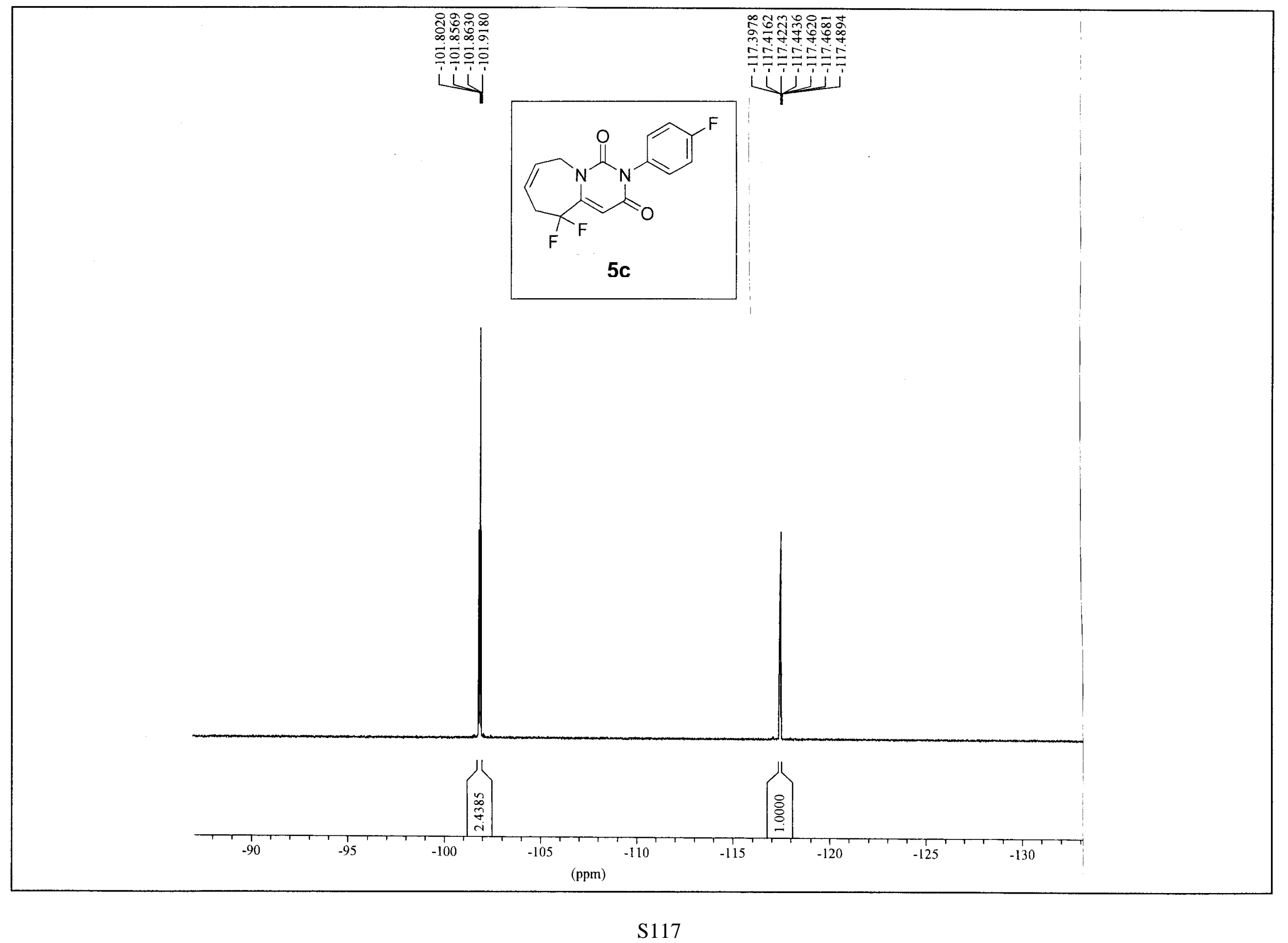




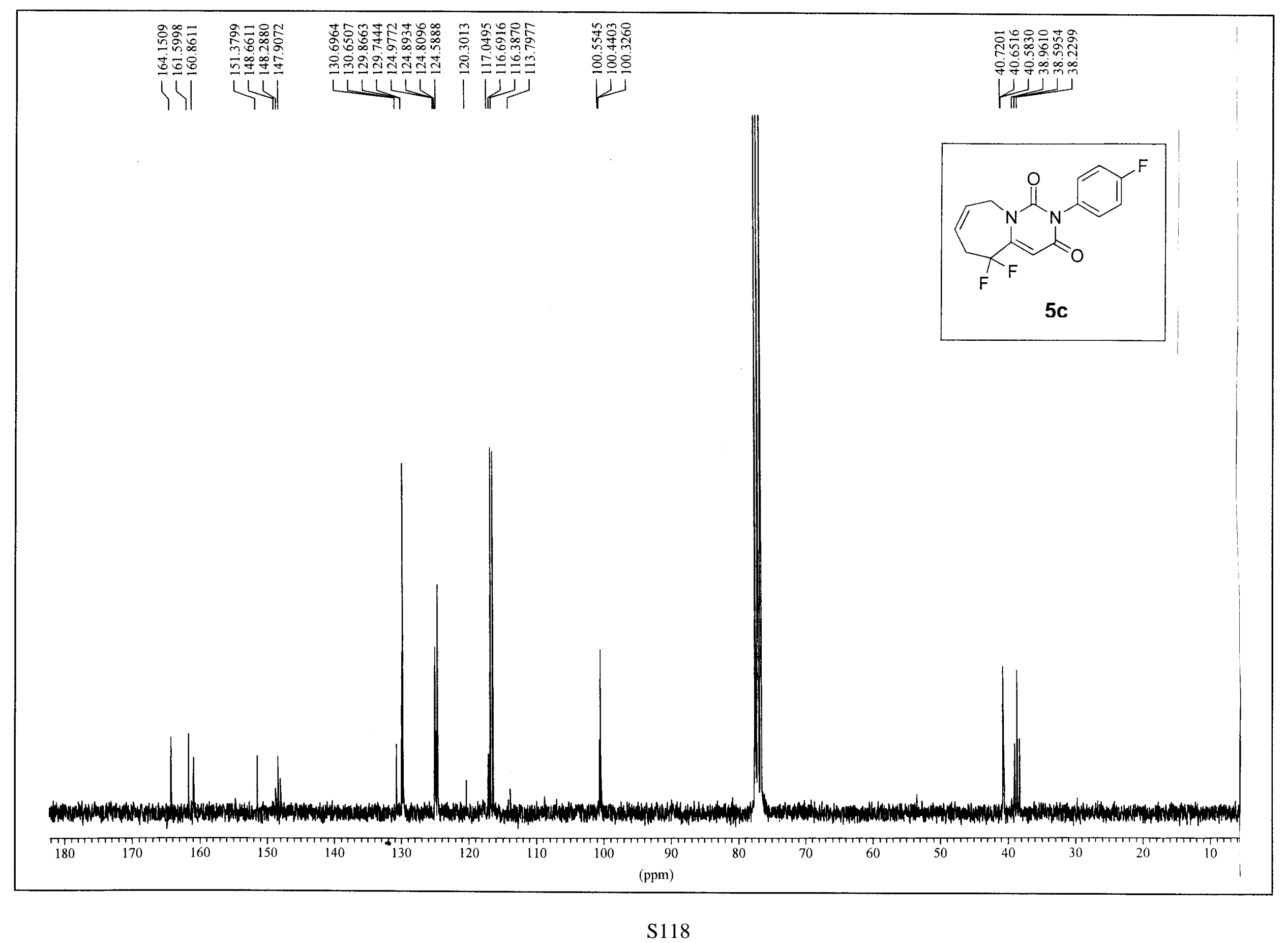




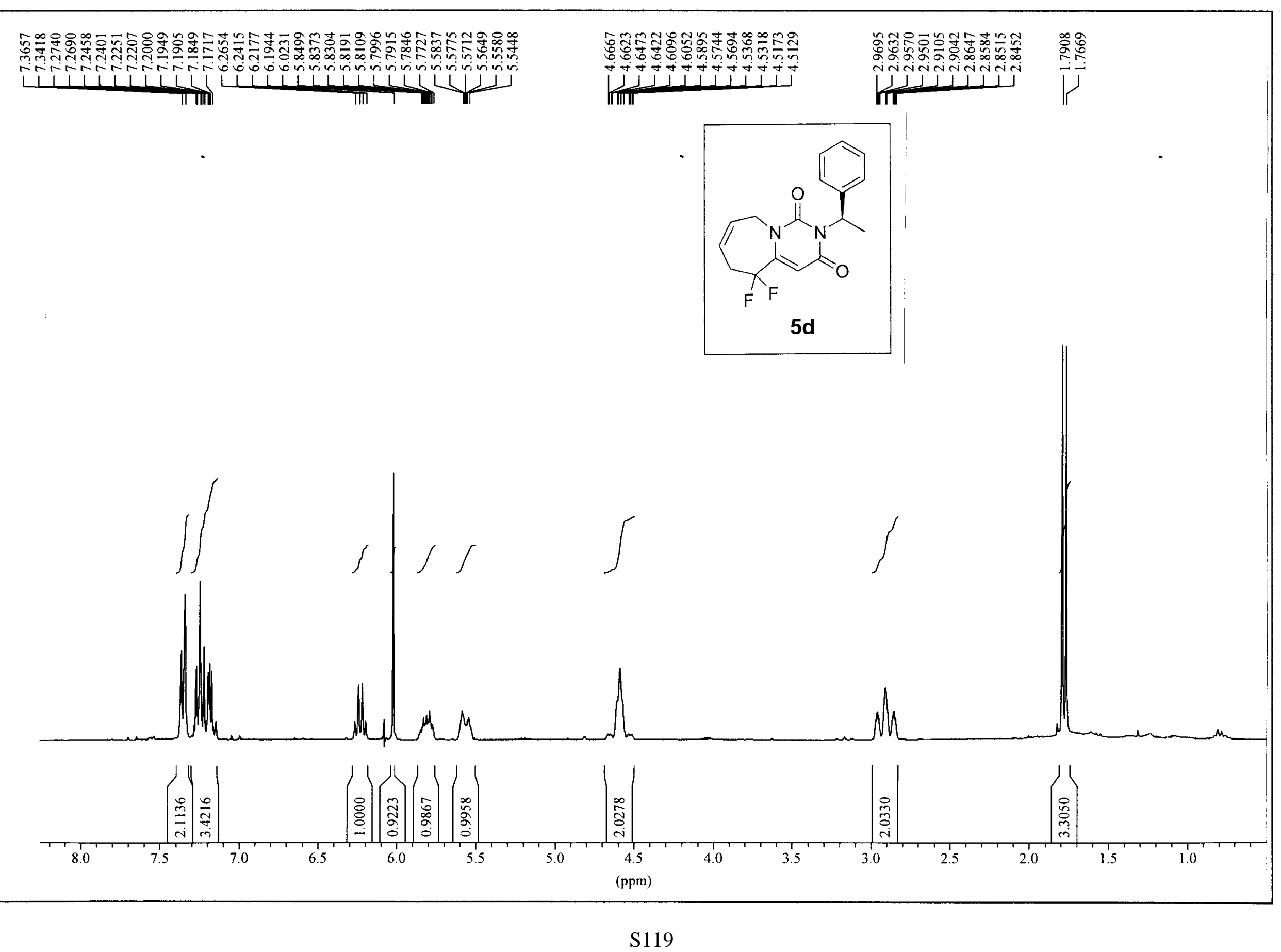




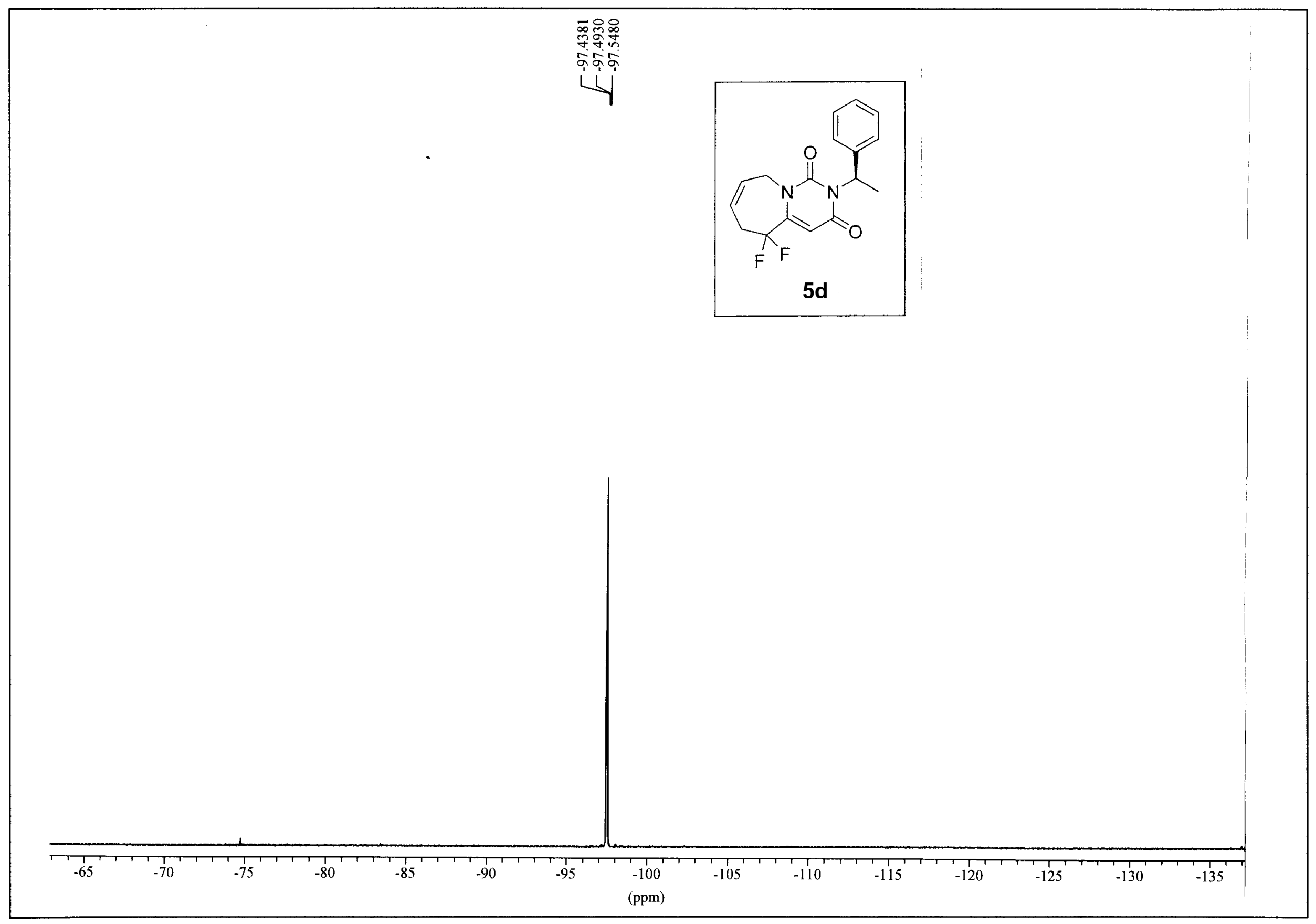




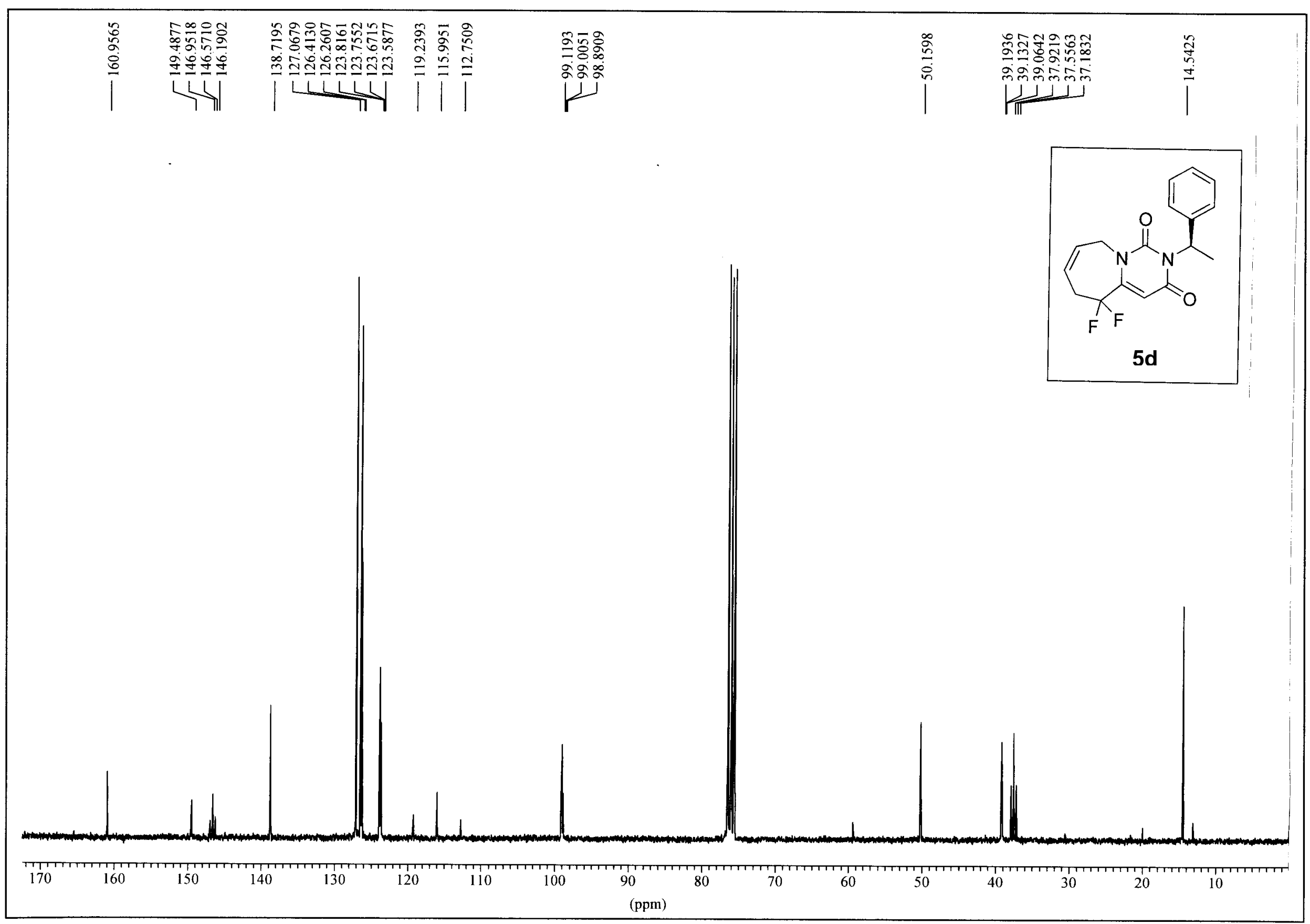

S121 


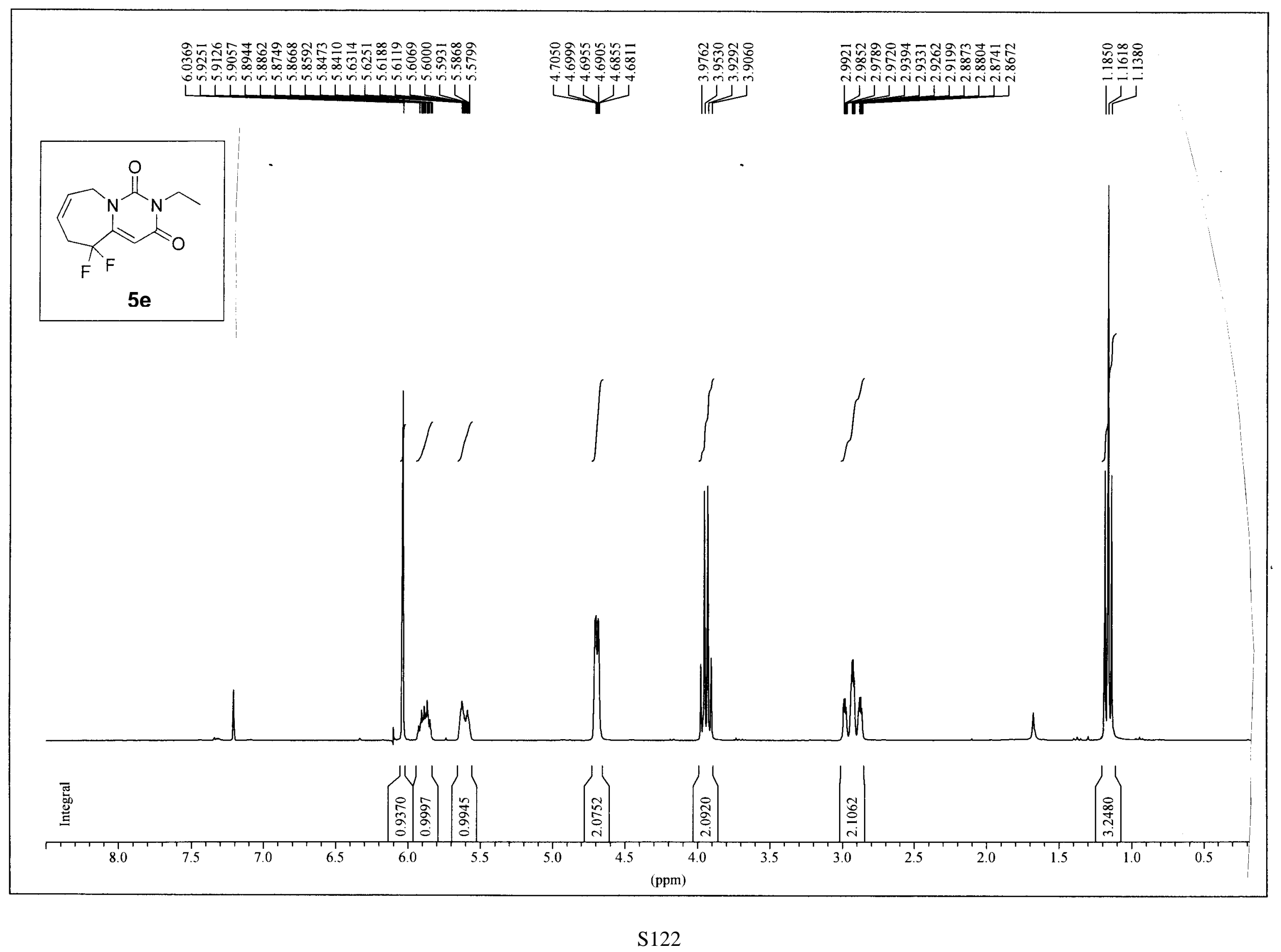




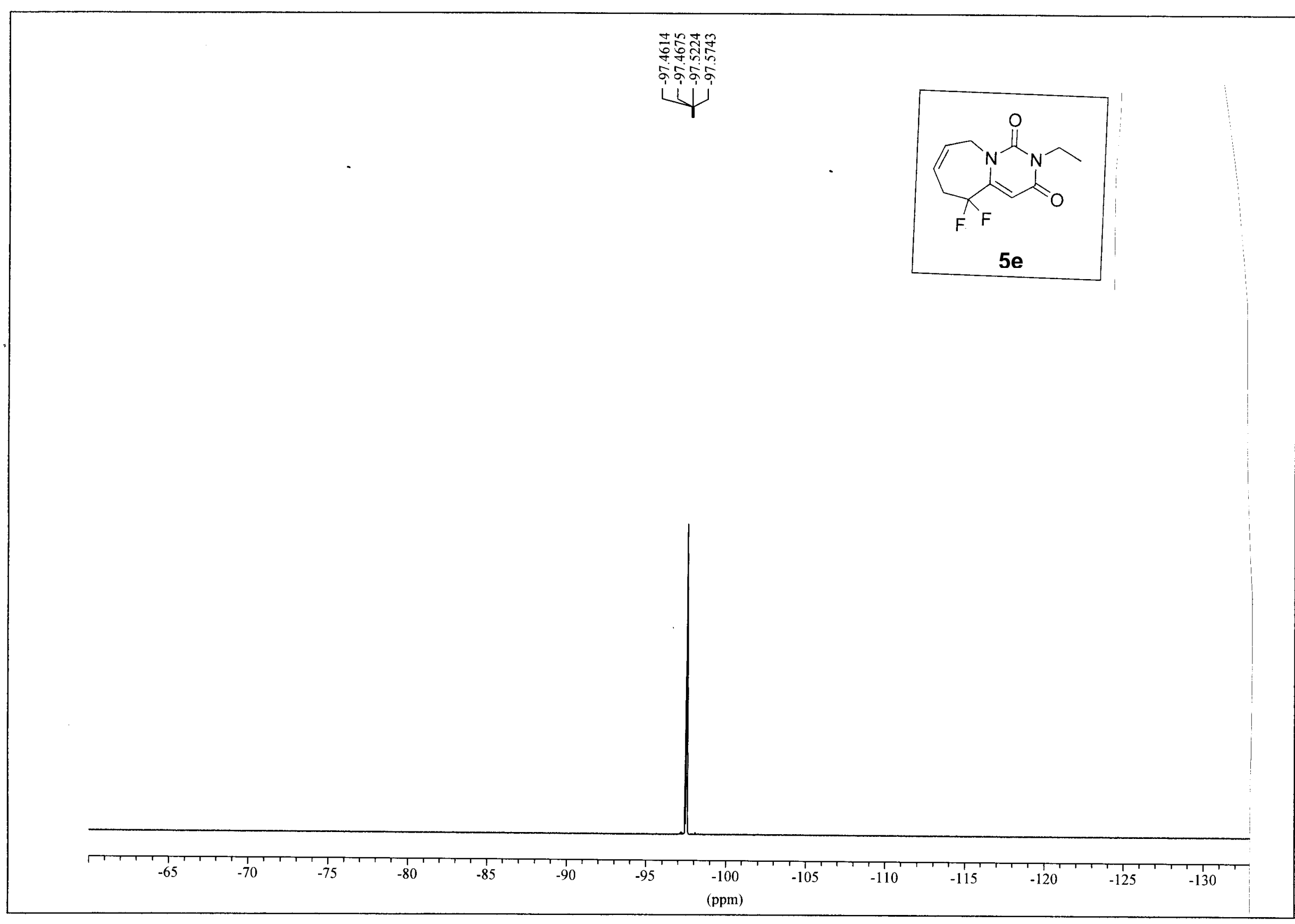




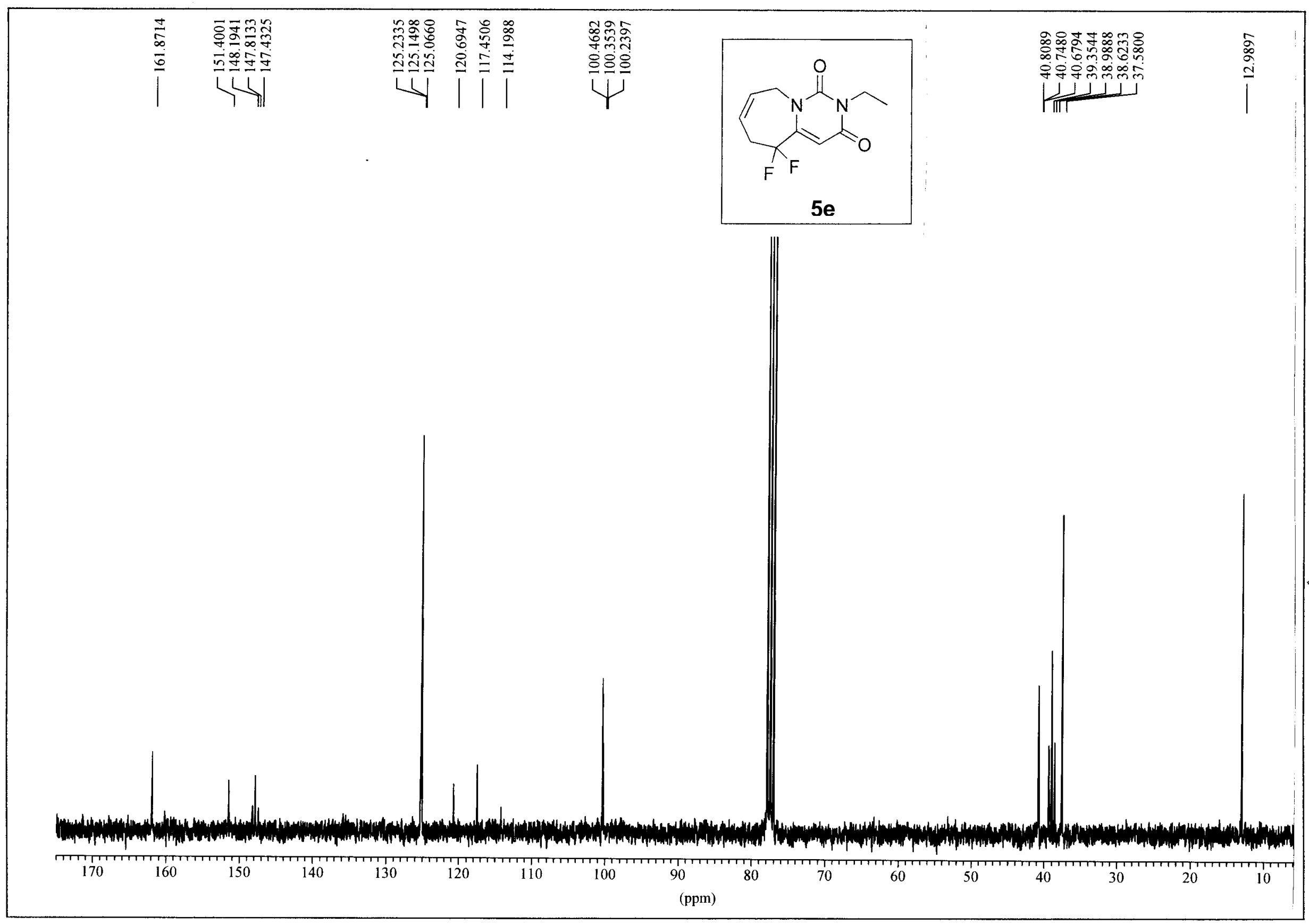



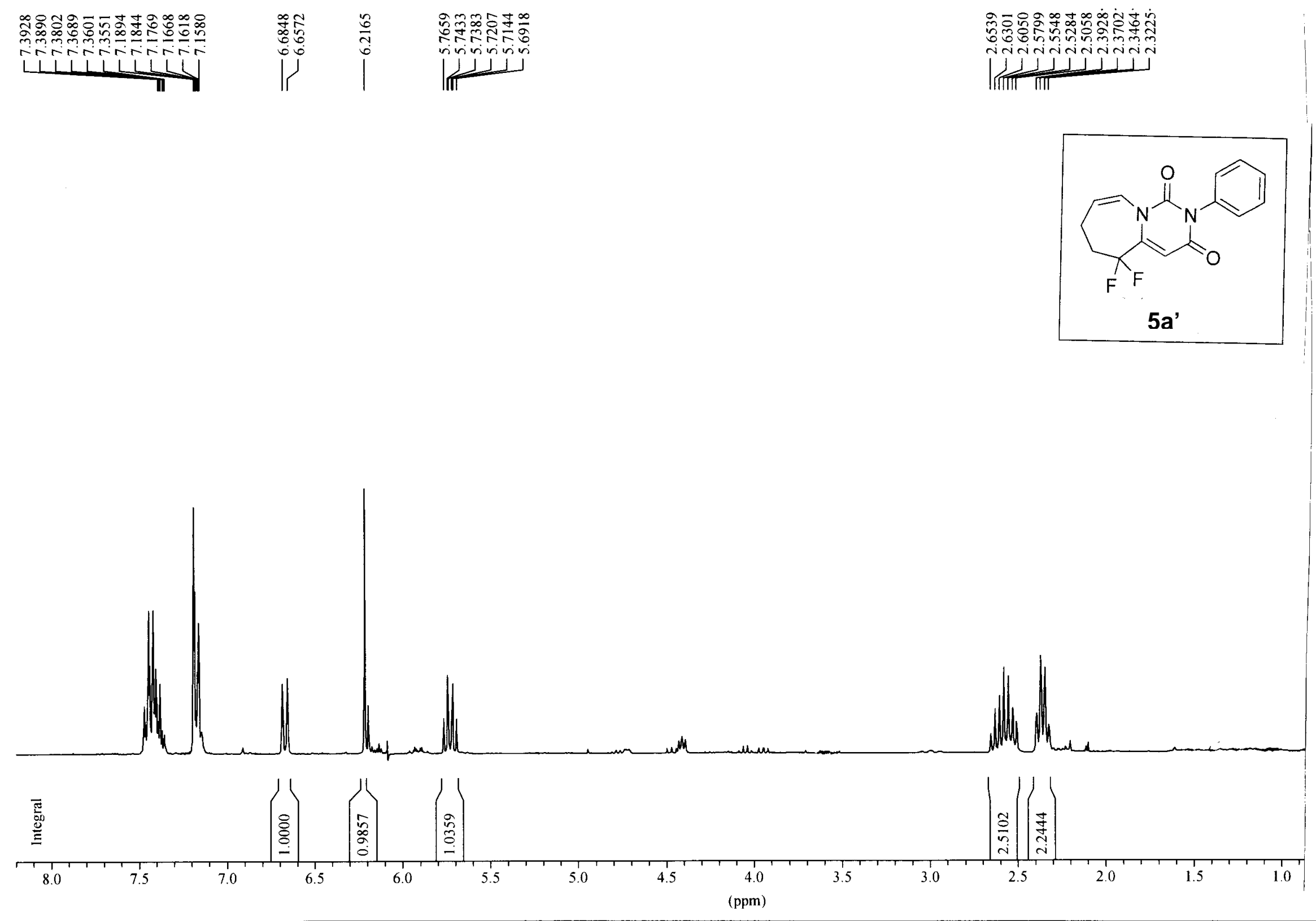


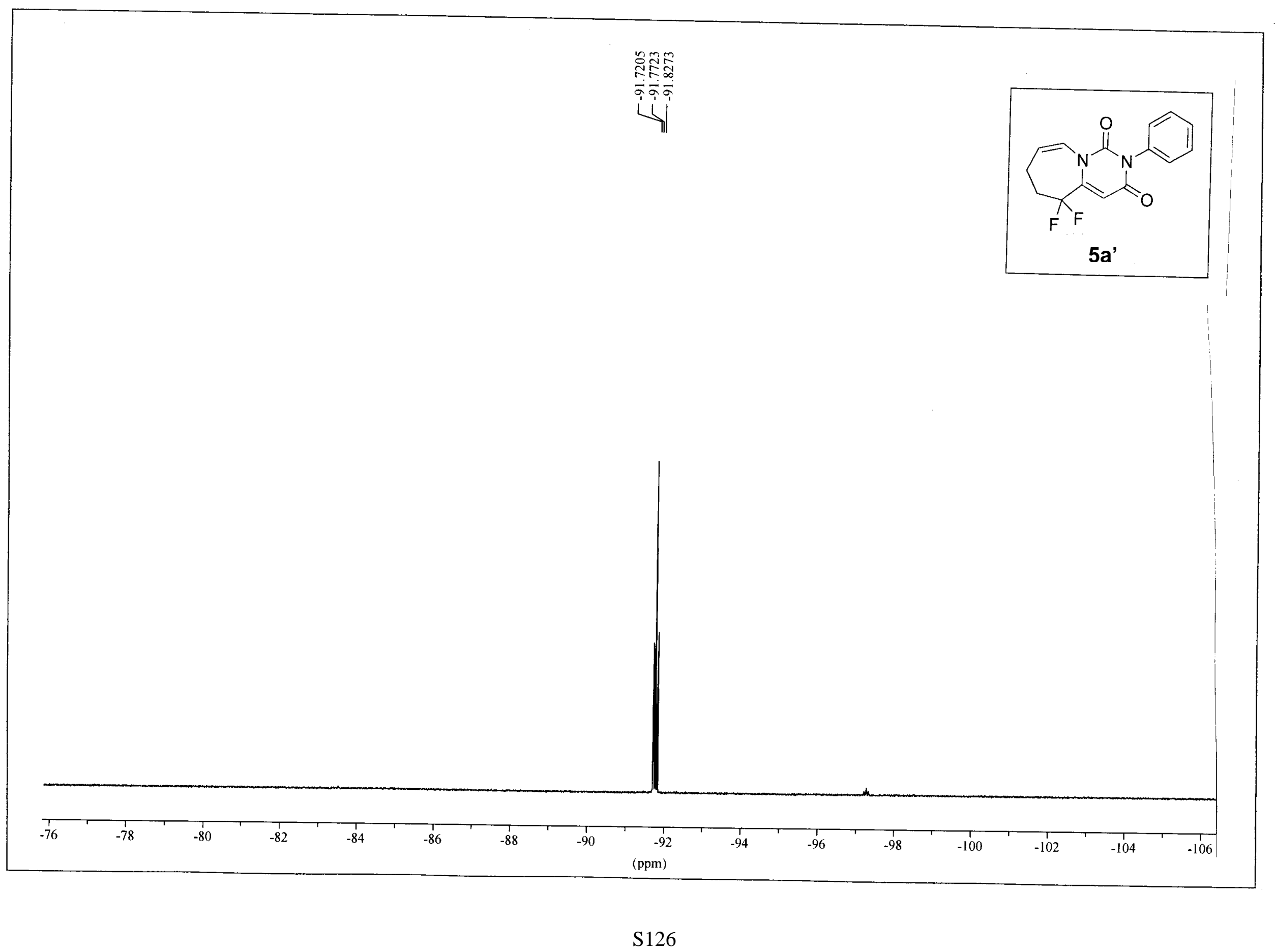




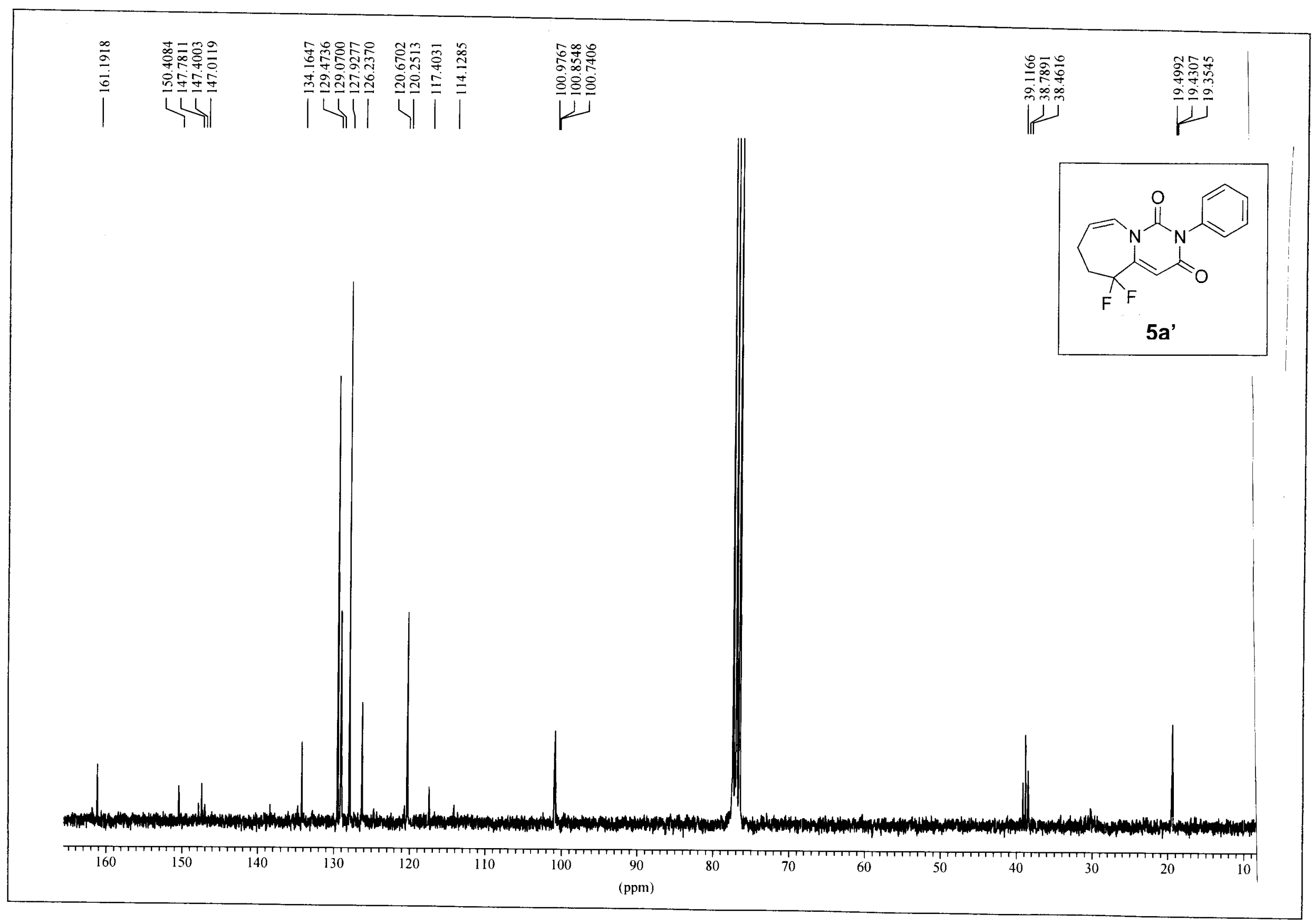

